Nova Southeastern University

2016

\title{
Using Diversity Ensembles with Time Limits to Handle Concept Drift
}

Robert M. Van Camp

Nova Southeastern University, waynevancamp@suddenlink.net

This document is a product of extensive research conducted at the Nova Southeastern University College of Engineering and Computing. For more information on research and degree programs at the NSU College of Engineering and Computing, please click here.

Follow this and additional works at: https://nsuworks.nova.edu/gscis_etd

Part of the Computer Sciences Commons

\section{Share Feedback About This Item}

\section{NSUWorks Citation}

Robert M. Van Camp. 2016. Using Diversity Ensembles with Time Limits to Handle Concept Drift. Doctoral dissertation. Nova Southeastern University. Retrieved from NSUWorks, College of Engineering and Computing. (984)

https://nsuworks.nova.edu/gscis_etd/984. 


\title{
Using Diversity Ensembles with Time Limits to Handle Concept Drift
}

\author{
by
}

Robert Van Camp

A dissertation report submitted in partial fulfillment of the requirements for the degree of Doctor of Philosophy

in

Computer Information Systems

Graduate School of Computer and Information Sciences

Nova Southeastern University 
We hereby certify that this dissertation, submitted by Robert Van Camp, conforms to acceptable standards and is fully adequate in scope and quality to fulfill the dissertation requirements for the degree of Doctor of Philosophy.

Sumitra Mukherjee, Ph.D

Date

Chairperson of Dissertation Committee

Michael J. Laszlo, Ph.D.

Dissertation Committee Member

Francisco J. Mitropoulos, Ph.D.

Date

Date

Approved:

Yong X. Tao, Ph.D., P.E., FASME

Date

Dean, College of Engineering and Computing

College of Engineering and Computing

Nova Southeastern University 
An Abstract of a Dissertation Report Submitted to Nova Southeastern University in Partial Fulfillment of the Requirements for the Degree of Doctor of Philosophy

\title{
Using Diversity Ensembles with Time Limits to Handle Concept Drift
}

\author{
by \\ Robert Van Camp \\ 2016
}

\begin{abstract}
While traditional supervised learning focuses on static datasets, an increasing amount of data comes in the form of streams, where data is continuous and typically processed only once. A common problem with data streams is that the underlying concept we are trying to learn can be constantly evolving. This concept drift has been of interest to researchers the last few years and there is a need for improved machine learning algorithms that are capable of dealing with concept drifts. A promising approach involves using an ensemble of a diverse set of classifiers. The constituent classifiers are re-trained when a concept drift is detected. Decisions regarding the number of classifiers to maintain and the frequency of re-training classifiers are critical factors that determine classification accuracy in the presence of concept drift. This dissertation systematically investigated these issues in order to develop an improved classifier for online ensemble learning. The impact of reducing the time requiring additional ensembles was studied using artificial and real world datasets. Findings from these studies revealed that in many cases the number of time steps additional ensembles are in memory can be reduced without sacrificing prequential accuracy. It was also found that this new ensemble approach performed well in the presence of false concept drift.
\end{abstract}




\section{Acknowledgements}

Completing my dissertation has been a rewarding personal experience for me. I believe going through this process will help me as I work with my students in the future. First, I would like to thank my advisor, Dr. Sumitra Mukherjee, for serving as my dissertation chair. Dr. Mukherjee consistently encouraged me along this path, from problem definition to dissertation defense. I greatly appreciate his help. In addition, my thanks to Dr. Michael Laszlo and Dr. Francisco Mitropoulos for their willingness to serve on my committee and provide valuable feedback.

Dr. Leandro Minku was gracious enough to provide the $\mathrm{C}$ code used for the original DDD algorithm. I appreciate his willingness to do so.

I would like to thank Marietta College for providing support for travel and for a sabbatical to aid in completing my dissertation. I would like to thank Dr. John Tynan and Dr. Mark Miller, my current and previous department chairs, for encouraging me in this process. I would like to thank my colleagues at Marietta College for words of support, especially those in my department.

I would also like to thank my children, Bethany Richetti and Will Van Camp, for their belief in me during these last six years. I wish the best for them in their studies. I also thank the rest of my extended family, including my mother-in-law Retta Wayne, who always asked how I was getting along with my research. My father-in-law, Wilson Wayne, is no longer here but was always a source of encouragement. While they are no longer here, I thank my father and mother, Don and Ruth Van Camp, they instilled in me a belief I could succeed in whatever I put my mind to. I know they would be happy and proud.

Finally, I would like to thank my wife, Mary Ellen. She has been my constant supporter in this dissertation process. She has been my proofreader, sounding board, and best friend. I could not have completed this dissertation process without her love and encouragement. 


\section{Table of Contents}
Abstract ii
List of Tables viii
List of Figures ix
List of Algorithms xi

\section{Chapters}

\section{Introduction 1}

Background 1

Problem Statement 8

Dissertation Goal 9

Relevance and Significance 9

Barriers and Issues 10

Assumptions, Limitations, and Delimitations 10

Summary 11

Definition of Terms 12

\section{Review of the Literature 15}

Overview 15

Justification of Review Criteria 15

Types of Machine Learning 16

Strategies for Supervised Learning 16

Definition of Concept Drift 17

Highlights in Concept Drift Research 19

Ensemble Methods with Concept Drift 19

Concept Drift Detection 20

EDDM 21

DWM 23 
Diversity with Ensembles 24

DDD Algorithm 26

False Positives in Concept Drift 27

Comparison of EDDM, DWM, and DDD 27

Performance Evaluation 28

Summary 29

\section{Methodology 31}

Overview of Methodology 31

Maximum Time Step Parameter 32

Testing with Artificial Datasets 32

Testing with Real World Data 36

Comparison with other Concept Drift Algorithms 38

Testing for False Positive Concept Drift 38

Preliminary Work 39

Detailed Test Plan 42

Resources Used 44

Summary 44

\section{Results 45}

Introduction 45

Determine Best MTS 46

Results of Comparison with other Concept Drift Algorithms 61

Results of Tests for False Positive Concept Drift 77

Summary of Results 91 


\section{Conclusions, Recommendations, and Summary 92}

Conclusions 92

Recommendations 93

Summary 94

\section{Appendices 96}

Appendix A - Comparison of Various MTS Settings 96

Appendix B - Frequency of MTS Values 105

Appendix C - Comparison of Best MTS, DDD, and DDD with Reset 107

Appendix D - Best MTS and Standard DDD, Average Time Steps in Four Ensembles 116

Appendix E - Comparison of Various W Settings using Best MTS 123

Appendix F - Comparison of Various $W$ Settings using Standard DDD 132

\section{References 141}




\section{List of Tables}

\section{Tables}

1. Examples of Severity 8

2. Definition of Terms 12

3. Severity for Artificial Datasets 34

4. Settings for Artificial Datasets 36

5. Settings for Real World Datasets 38

6. Parameters for CreateBatchFile.py 41 


\section{List of Figures}

\section{Figures}

1. Concept Drift Detection with DDD 5

2. Types of Drifts 18

3. Concept Drift Detection with EDDM 22

4. Circle - Low Severity/High Speed, MTS Comparison 47

5. Circle - Low Severity/Low Speed, MTS Comparison 48

6. Circle - Low Severity/Low Speed, MTS Comparison (Magnified) 49

7. Circle - High Severity/High Speed, MTS Comparison 50

8. Circle - High Severity/High Speed, MTS Comparison (Magnified) 51

9. Circle - High Severity/Low Speed, MTS Comparison 52

10. SineV - High Severity/Medium Speed, MTS Comparison 53

11. SineH - High Severity/Low Speed, MTS Comparison 54

12. Plane - High Severity/Medium Speed, MTS Comparison 55

13. Boolean - High Severity/High Speed, MTS Comparison 56

14. Frequency of MTS Values for Artificial Data 57

15. Electricity, MTS Comparison 58

16. Forest CoverType, MTS Comparison 59

17. KDD 1999, MTS Comparison 60

18. Circle - Low Severity/High Speed, Comparison of Algorithms 62

19. Circle - Low Severity/Low Speed, Comparison of Algorithms 63

20. Circle - High Severity/High Speed, Comparison of Algorithms 64

21. Circle - High Severity/Low Speed, Comparison of Algorithms 65

22. SineV - Medium Severity/High Speed, Comparison of Algorithms 66 
23. Line - Medium Severity/High Speed, Comparison of Algorithms 67

24. Plane - High Severity/Medium Speed, Comparison of Algorithms 68

25. Boolean - Medium Severity/High Speed, Comparison of Algorithms 69

26. SineH - Low Severity/Low Speed, Comparison of Algorithms 70

27. Circle - Comparison of Average Number of Time Steps in Four Ensembles 72

28. Electricity - Comparison of Algorithms 73

29. Forest CoverType - Comparison of Algorithms 74

30. KDD 1999 - Comparison of Algorithms 75

31. Real World Data - Average Number of Time Steps in Four Ensembles 77

32. Circle - Low Severity/High Speed, Comparison of $W$ values 79

33. Circle - Low Severity/Low Speed, Comparison of $W$ values 80

34. Circle - High Severity/High Speed, Comparison of $W$ values 81

35. Circle - High Severity/Low Speed, Comparison of $W$ values 82

36. SineV - Medium Severity/Low Speed, Comparison of $W$ values 83

37. SineH - Low Severity/High Speed, Comparison of $W$ values 84

38. SineH - Medium Severity/Low Speed, Comparison of $W$ values 85

39. Line - Medium Severity/High Speed, Comparison of $W$ values 86

40. Plane - High Severity/Medium Speed, Comparison of $W$ values 87

41. Electricity - Comparison of $W$ values 88

42. Forest CoverType - Comparison of $W$ values 89

43. KDD 1999 - Comparison of $W$ values 90 


\section{List of Algorithms}

\section{Algorithms}

1. An overview of the DDD algorithm 6

2. An overview of the DWM algorithm 23

3. Modified Online Bagging 25 


\section{Chapter 1}

\section{Introduction}

\section{Background}

Supervised machine learning methods are used to determine relationships between input and output variables based on available observations. The goal is to come up with a function able to predict the output based on the given inputs. When the output variable is categorical, the learned function is said to be a classifier. Traditionally, classifiers are trained in a batch mode using all available observations. In recent years online learning techniques have been developed to deal with applications where observations become available sequentially, one at a time. Restrictions on time and on computing resources prevent machine learning methods to store and process more than a limited number of observations in a batch mode. The applications for online learning have grown in recent years and include such areas as credit card transaction flows, computer security, industrial process control, and intelligent user interfaces. In these systems, data tends to occur in a continuous stream, making storage and repeated processing difficult.

In online learning, the underlying relationships between the input and the output variables may change over time. This is called concept drift. A concept may be characterized as a joint distribution of the input and output variables. A change in this joint distribution is characterized as a concept drift.

Among the most successful methods to deal with concept drift in online learning is the one proposed by Minku and Yao (2012). Their method uses multiple ensembles, each consisting of online classifiers; the predicted class is the mode class or a weighted average of the constituent classifiers in the ensembles. Using a set of diverse classifiers 
with different strengths and weaknesses resulted in the classification accuracy of the ensembles to be significantly greater than any of its constituent classifiers.

The work of Minku and Yao (2012) focused on the use of diversity in ensembles to provide improved accuracy in the presence of concept drift. Since concept drift can have different speeds and severities, it was found that multiple ensembles of varying diversities provided superior accuracy to other drift handling approaches. Their approach improved upon previous methods, but required two ensembles before concept drift was detected (when the concept is stable) and four ensembles after concept drift occurred. Computational overheads are associated with maintaining ensembles. Since an additional two ensembles were required after concept drift, the question of how long these two additional ensembles should remain in memory deserves further investigation. This dissertation will systematically investigate how decisions regarding maintenance of additional ensembles affect the accuracy of ensemble classifiers. The rest of this section discusses the role of diversity in online ensemble classifiers and introduces the algorithm developed by Minku and Yao (2012).

For this study, online learning systems are ones where training examples are processed once on arrival and are not stored. A current hypothesis representing all training instances so far can be maintained by the system. A hypothesis is a function that maps the input variable to an output class. Hypotheses are updated as new training observations arrive. This approach is described by Oza and Russell (2001) and Fern and Given (2003); details are provided in the literature review section.

Work by Fern and Givan (2003) showed how ensembles of small trees provided improvement in classification accuracy over a single tree. Wang, Fan, Yu, and Han (2003) demonstrated the error reduction property and showed that a classifier ensemble can improve upon a single classifier when concept drift is present. Their research found that an ensemble classifier can reduce classification error by making the weight of the 
classifier inversely proportional to the expected error of the classifier. Research by Oza and Russell (2001) produced online versions of the ensemble methods bagging and boosting, allowing the benefits of ensemble learning to extend to an online environment.

There are two major approaches for using ensemble methods to detect concept drift. The first approach includes a mechanism to detect drifts explicitly. Here a measure is typically used to determine if a concept drift has occurred. If a concept drift is detected, a new online ensemble of classifiers is created and all classifiers in the ensemble are retrained. This approach tends to react quickly to concept drift if it is found early. One example of this technique is the work by Baena-García, Del Campo-Ávila, Fidalgo, and Bifet (2006), called the Early Drift Detection Method (EDDM). With this approach it is assumed that the difference in time between consecutive errors will increase when a stable concept is in the process of being learned. A noticeable drop in this difference in time between consecutive errors is considered a concept drift. A new classifier system is generated at this point.

The second approach handles concept drift implicitly. Here weights are assigned to the classifiers of each ensemble and these weights are based on accuracy of the classifiers. Representative of this approach is the work of Kolter and Maloof (2007). Their Dynamic Weighted Majority (DWM) ensemble set up a group of weighted classifiers. Classifiers were added and deleted based on the effectiveness of those classifiers. Classifier weight was reduced if an example was misclassified. In addition, the classifiers which performed poorly were removed from the ensemble if their weights fell below a predefined threshold.

Minku, White, and Yao (2010) discussed the use of diversity to aid online learning where concept drift was found. Their study indicated that a range of diversity levels used with old and new concepts allowed for improved generalization. These diversity levels reflect the degree of agreement between constituent classifiers in the 
ensemble. When pairs of classifiers tend to agree, these classifiers are considered less diverse. This study revealed that diversity aided in reduction of error at the beginning of concept drift but did not help improve long-term recovery from concept drift.

The work by Minku and Yao (2012) found that different levels of ensemble diversity used with old and new concepts allowed for improved generalization and gave the maximum prequential accuracy. Prequential accuracy is defined by Dawid and Vovk (1999) as the average accuracy computed from each example presented for training, prior to the example being learned. Prequential accuracy assumes that prediction can be improved by mapping the prediction to a one-step ahead forecasting system.

It has been shown (Minku \& Yao, 2012) that old concept knowledge is useful in new concept learning. They found that high diversity ensembles trained on the old concept could converge to the new concept when the learning of the new concept occurred with low diversity.

The timeline in Figure 1 illustrates their approach to concept drift. As shown, two ensembles are created, one with low diversity and the other with high diversity. After concept drift is detected, two additional ensembles are maintained until the concept is stable. At this point only two ensembles are used. 
Figure 1. Concept Drift Detection with DDD

$\mathrm{h}_{\mathrm{nl}}-$ new low diversity ensemble

$\mathrm{h}_{\mathrm{ol}}$ - old low diversity ensemble

$\mathrm{h}_{\text {nh }}-$ new high diversity ensemble

$\mathrm{h}_{\mathrm{oh}}$ - old high diversity ensemble

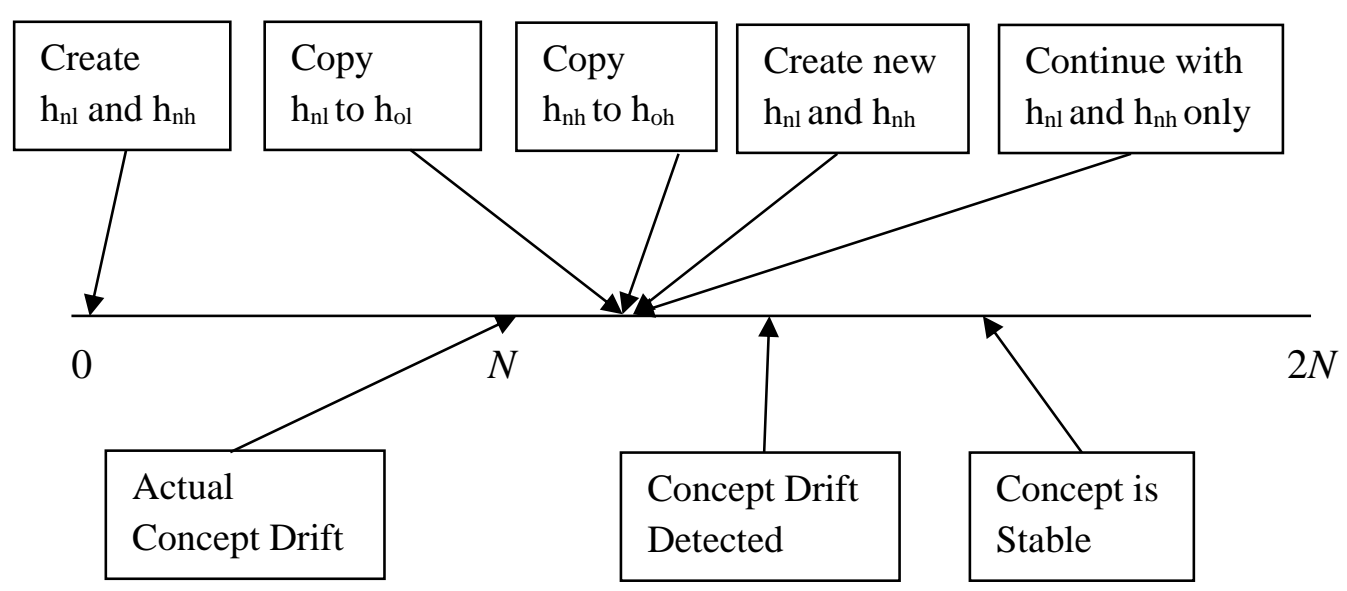

Figure 1. Timeline showing how the number of ensembles changes from two to four when concept drift is detected. Data transmission begins at time 0 , concept drift occurs at time $N$, and data transmission is completed at time $2 N$.

Minku and Yao (2012) developed an algorithm called Diversity for Dealing with Drifts (DDD) as an online ensemble learning approach. The DDD algorithm is summarized in Algorithm 1. 


\section{Algorithm 1. An overview of the DDD algorithm}

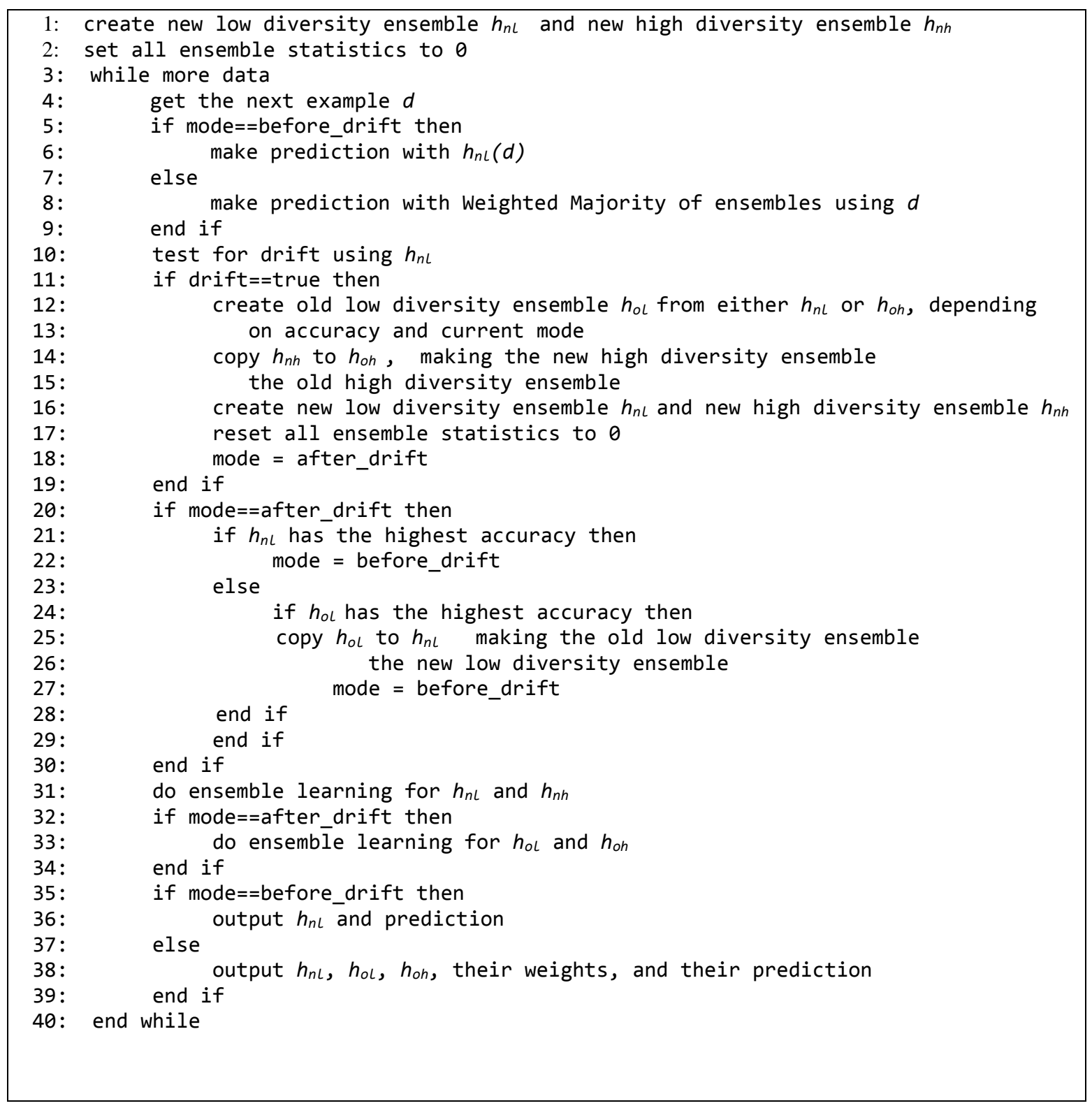

Algorithm 1 shows that only two ensembles are created in before-drift-mode (line 1). These ensembles are the new low diversity ensemble and the new high diversity ensemble. Predictions in DDD are made with the new low diversity ensemble in beforedrift-mode and with a weighted majority of ensembles when in after-drift-mode (lines 59). When concept drift is detected, two additional ensembles join the system, one with low diversity and the other with high diversity (line 16). Also, when a drift is detected, the algorithm assigns the old low diversity ensemble used prior to drift detection either to 
the new low diversity ensemble or the old high diversity ensemble, choosing the one with the highest accuracy (lines 12-13). In before-drift- mode, ensemble learning occurs only with the two ensembles initially created, one with low diversity and the other with high diversity (line 31). After the concept drift is detected and until the concept is stable, all four ensembles do ensemble learning (lines 31-34). In each time step the output of the learner is displayed. In before-drift-mode, this output comes from the new low diversity ensemble (line 36). In after-drift-mode, the output is weighted by the new low diversity ensemble, the old low diversity ensemble, and the old high diversity ensemble (line 38). While the DDD algorithm has a minimum for the number of time steps to keep four ensembles after a drift is detected, a parameter limiting the maximum number of time steps all four ensembles are in memory is not implemented in the algorithm. The addition of this parameter and its role in the improvement of classifier accuracy was the major focus of this dissertation.

The DDD algorithm was tested with both real world and artificial data. The artificial data contained low, medium, and high severity and low, medium, and high speed. Severity is defined as the percentage of input data that has its target class modified when the drift is complete. Table 1 shows severity for the artificial datasets Circle and SineV used by Minku and Yao (2012). In the dataset Circle, for example, $16 \%$ of the input data had its target class modified when the radius $r$ changed from .2 to .3 in the Circle formula, representing low severity. Likewise, $38 \%$ of the input data had its target class modified when $r$ changed from .2 to .4 in the Circle formula, indicating medium severity. 
Table 1. Examples of Severity

\begin{tabular}{|c|c|c|c|l|}
\hline Problem & Equation & $\begin{array}{c}\text { Fixed } \\
\text { Values }\end{array}$ & $\begin{array}{c}\text { Before } \rightarrow \text { After } \\
\text { Drift }\end{array}$ & \multicolumn{1}{c|}{ Severity } \\
\hline Circle & $(x-a)^{2}+(y-b)^{2} \leq$ & $a=.5$ & $r=.2 \rightarrow .3$ & $16 \%$ Low \\
& $r^{2}$ & $b=.5$ & $r=.2 \rightarrow .4$ & $38 \%$ Medium \\
& & & $r=.2 \rightarrow .5$ & $66 \%$ High \\
\hline SineV & $y \leq a \sin (b x+c)+d$ & $a=1$ & $d=-2 \rightarrow 1$ & $15 \%$ Low \\
& & $b=1$ & $d=-2 \rightarrow 4$ & $45 \%$ Medium \\
& & $\mathrm{c}=0$ & $d=-2 \rightarrow 7$ & $75 \%$ High \\
\hline
\end{tabular}

Drifting time is the number of time steps needed for the new concept to take the place of the old one. The inverse of drifting time is the speed of the concept drift. Speed was modeled by the degree of dominance of the new and old concepts defined by Narasimhamurthy and Kuncheva (2007). Drifting time took on the values 1, 0.25N, and $0.5 N$ to allow for the creation of fast, medium, and slow speeds. Minku and Yao (2012) joined $\{$ low, medium, high $\}$ speed with $\{$ low, medium, high $\}$ severity to create nine ways to test for varying types of concept drift.

\section{Problem Statement}

An unanswered question relates to the tradeoff between improved accuracy and the increased resources necessary to maintain the four ensembles. Is it necessary to maintain the four ensembles the entire time between drift detection and the return to a stable mode? Specifically, how do the number of time steps in the after drift detection mode affect the performance of an online ensemble classifier? Is it possible to maintain the four ensembles in DDD a shorter time to improve resource usage while maintaining prequential accuracy? 


\section{Dissertation Goal}

In order to study these questions in detail, a parameter which controls the maximum number of time steps the four ensembles are maintained was introduced. A range of values for this parameter was studied. The drift conditions tested reflected those of Minku and Yao (2012) and included both artificial and real world test data. Artificial data was broken down by speed and severity as was described in the introduction. Real world datasets were analyzed in a manner similar to that of Minku and Yao (2012).

\section{Relevance and Significance}

As stated earlier, online learning applications have grown in recent years and include such areas as credit card transaction flows, computer security, industrial process control, and intelligent user interfaces. Part of an intelligent user interface could be access

to streaming news. The survey by Gama, Žliobaite, Bifet, Pechenizkiy, and Bouchachia (2014) gives the example that while incoming news items might not change, the distribution of news items found to be interesting and not interesting for a specific user can change. This is an example of concept drift. This survey also noted that the amount of concept drift research has greatly increased in the last ten years, illustrating the importance of concept drift in online learning.

The research I built on (Minku \& Yao, 2012) is mentioned as a "notable technique" in the Gama et al. (2014) survey. Minku and Yao (2012) build on earlier successful concept drift detection techniques giving improved or comparable results, depending on the severity and the speed of the concept drift. 


\section{Barriers and Issues}

The survey on concept drift by Gama et al. (2014) mentions that while interest in concept drift is growing, the appearance of concept drift in multiple problem domains creates an inconsistency in terminology and techniques. Research has also found that concept drift can vary both by the severity of the drift and by the speed at which the drift occurs. Minku and Yao (2012) used low, medium, and high severity training sets along with low, medium, and high speed training sets to study the impact of low and high diversity ensembles with concept drift.

Also, it is hard to predict if and when concept drift will occur. To aid with this prediction, artificial datasets with build-in concept drift are used for analysis purposes. To help confirm results, real world datasets are also used. An additional issue is the possibility of the detection of concept drift where it does not exist, a false positive. This dissertation attempted to improve on or equal the accuracy of the DDD algorithm of Minku and Yao (2012) with the addition of a maximum on the number of time steps four ensembles used. This maximum on the number of time steps was also studied in situations where false concept drift was present.

\section{Assumptions, Limitations, and Delimitations}

The focus of this study is online supervised learning using classification (having discrete outcomes), as opposed to regression (having continuous outcomes). Though the study is restricted to classification, it is believed that results can also be extended to regression. The goal in the study is to make improvements in algorithm accuracy, not speed. Since speed will not be examined, multiple computers will be used in the testing phase. 


\section{Summary}

In summary, the advantages of maintaining four ensembles after concept drift was detected have been shown by Minku and Yao (2012). These advantages include robust accuracy detection for a variety of drift types and excellent accuracy in the absence of concept drift and when false positive concept drifts appear. Since the benefits of using four ensembles is apparent but more resource intensive than EDDM and DWM, a parameter to control the maximum number of time steps the four ensembles are in memory will help provide an answer to the question of how long the four ensembles need to be present to provide good accuracy. 


\section{Definition of Terms}

Table 2. Definition of Terms

\begin{tabular}{|c|c|}
\hline Term & Definition \\
\hline Classification & Supervised learning with a categorical variable \\
\hline Controlled Permutation & Using randomized copies as in cross-validation \\
\hline DDD & Diversity for Dealing with Drifts algorithm \\
\hline Diversity levels & $\begin{array}{l}\text { Degree of agreement between constituent } \\
\text { classifiers in the ensemble }\end{array}$ \\
\hline Drifting Time & $\begin{array}{l}\text { Number of time steps needed for the new concept } \\
\text { to take the place of the old one }\end{array}$ \\
\hline DWM & Dynamic Weighted Majority \\
\hline EDDM & Early Drift Detection Method \\
\hline Holdout & $\begin{array}{l}\text { A subset of training and testing data is used for } \\
\text { testing }\end{array}$ \\
\hline Incremental Learning & Processes occurring in batches \\
\hline ITI & Incremental Tree Inducer \\
\hline kappa-statistic & Measures the accuracy of an intelligent classifier \\
\hline$M T S$ & Maximum Time Step parameter \\
\hline
\end{tabular}




\begin{tabular}{|c|c|}
\hline Term & Definition \\
\hline Online Bagging & $\begin{array}{l}\text { Training ensembles by sending } K \text { copies of the } \\
\text { new example, based on a Poisson distribution }\end{array}$ \\
\hline Online Learning & $\begin{array}{l}\text { All training data does not need to be available at } \\
\text { the beginning }\end{array}$ \\
\hline Poisson Distribution & $\begin{array}{l}\text { A discrete probability distribution used in DDD } \\
\text { to create diversity in ensembles }\end{array}$ \\
\hline Prequential Accuracy & $\begin{array}{l}\text { Average accuracy of predicted examples, prior to } \\
\text { the examples being learned }\end{array}$ \\
\hline Q Statistic & Measures diversity in ensembles \\
\hline RAM-hours & $\begin{array}{l}\text { Computed from the rental cost in a cloud- } \\
\text { computing environment }\end{array}$ \\
\hline Real Concept Drift & A change in the probability of a class occurring \\
\hline Regression & $\begin{array}{l}\text { Supervised learning with a continuous output } \\
\text { variable }\end{array}$ \\
\hline ROC curves & Receiver Operating Characteristic curves \\
\hline $\begin{array}{l}\text { Semi-Supervised } \\
\text { Learning }\end{array}$ & $\begin{array}{l}\text { Uses a combination of labeled and unlabeled } \\
\text { examples for learning }\end{array}$ \\
\hline Severity & $\begin{array}{l}\text { Percentage of input data that has its target class } \\
\text { modified when the drift is complete }\end{array}$ \\
\hline Speed & The inverse of drifting time \\
\hline Stream Learning & Processes incoming data sequentially \\
\hline
\end{tabular}




\begin{tabular}{|c|c|}
\hline Term & Definition \\
\hline Unsupervised Learning & Does not have labeled data in its training data \\
\hline Virtual Concept Drift & $\begin{array}{l}\text { The input data changes but the boundary between } \\
\text { class labels does not change }\end{array}$ \\
\hline$W$ & $\begin{array}{l}\text { Multiplier on the weight of the old low diversity } \\
\text { ensemble, used for false positive concept drift }\end{array}$ \\
\hline
\end{tabular}




\section{Chapter 2}

\section{Review of the Literature}

\section{Overview}

This section begins with a justification of the criteria for the research included and excluded in the review. Then background for machine learning, supervised learning, and concept drift will be presented. After that the highlights of concept drift research will be given, followed by key work where ensembles were used to handle concept drift. Then research that has been done by adding diversity in ensembles to handle concept drift will be shown. Following will be a discussion of work done on how to minimize the impact of false positive concept drift on classifier accuracy. Finally, a review of common performance evaluation techniques used in concept drift research will be presented.

\section{Justification of Review Criteria}

As shown in the survey by Gama et al. (2014), research in the area of concept drift research is strong and growing, but it is also fragmented into different problem domains. Also, there is disagreement among researchers in this field regarding terminology. Since concept drift research covers a broad area, this review will not be exhaustive. Beyond the highlights of concept drift research, the primary criteria for this review will be how close specific research papers are to my dissertation topic. For example, research on the memory of a predictive model is related to concept drift but falls outside of my research focus, as does work on reoccurring concept management. Included would be work done on concept drift detection and learning. 


\section{Types of Machine Learning}

The goal in machine learning is for computer programs to automatically find patterns and learn to recognize concepts from data (Han, Kamer, \& Pei, 2012). The three major types of machine learning are supervised learning, unsupervised learning, and semi-supervised learning. Supervised learning has labeled data in its training data so that as training occurs, the correct response is available. If the target to predict is a categorical variable, this process is called classification. If the variable is continuous the process is called regression. With unsupervised learning, the class labels are not known. An unsupervised learning model identifies clusters of data and therefore creates its own class labels. Semi-supervised learning uses a combination of labeled and unlabeled examples for learning. For this dissertation, supervised learning using classification will be used. Duda, Hart, and Stork (2001) describe classification using Bayesian Decision Theory through the prior probabilities for classes $p(y)$ and the class conditional probability $p(X \mid y)$. These values are used to compute the posterior probability of the classes, given by:

$$
p(X \mid y)=\frac{p(y) p(X \mid y)}{p(X)}
$$

Here $p(X)$ is defined as:

$$
\sum_{y=1}^{c} p(y) p(X \mid y)
$$

where $c$ is the number of output classes, $X$ is the input value, and $y$ is the class label.

\section{Strategies for Supervised Learning}

Broad categories of supervised learning are offline learning and online learning. In offline learning, the entire training set needs to be available before predictions can be 
made. With online learning, all of the training data does not need be available at the beginning. With online learning the learning model is updated as more training data enters the system. Variations of online learning include incremental learning and stream learning. Incremental learning is defined by processes occurring in batches, providing a way to not have the entire training set available like offline learning and without including the restriction of sequential processing found in online learning. With incremental learning, the system may be updated by referring to previous examples. Finally, stream learning algorithms process incoming data sequentially like online learning but the data is also continuous and high speed. Because of these requirements, stream learning algorithms must perform with low memory and low processing time. The focus of this dissertation will be online learning where data enters the system in the form of a data stream.

\section{Definition of Concept Drift}

In situations where data streams occur, the underlying data distribution can change. For example, Kolter and Maloof (2007) use the example of a professor's email classification system. The types of email identified as "important" and "not important" will change as semesters change and as conference deadlines come and go. When these class labels change over time, this is considered concept drift.

Mathematically, concept drift can be defined as:

$$
\exists X: p_{t_{0}}(X, y) \neq p_{t_{1}}(X, y)
$$


where $p_{t_{0}}$ is the joint distribution at time $t_{0}$ of the input set $X$ and the target $y$. Kelly, Hand, and Adams (1999) mention that concept drift can change the probabilities of classes $p(y \mid X)$, leading to misclassification of the target variable $y$. Specifically, this change is called real concept drift (Gama et al., 2014). A second kind of drift called virtual concept drift is defined as a change in $p(X)$ without a change in $p(y \mid X)$ (Tsymbal, A., 2004; Widmer \& Kubat, 1993). Figure 2, from Gama et al. (2014), shows how class boundaries and labels change during real concept drift and virtual concept drift.

Figure 2. Types of Drifts
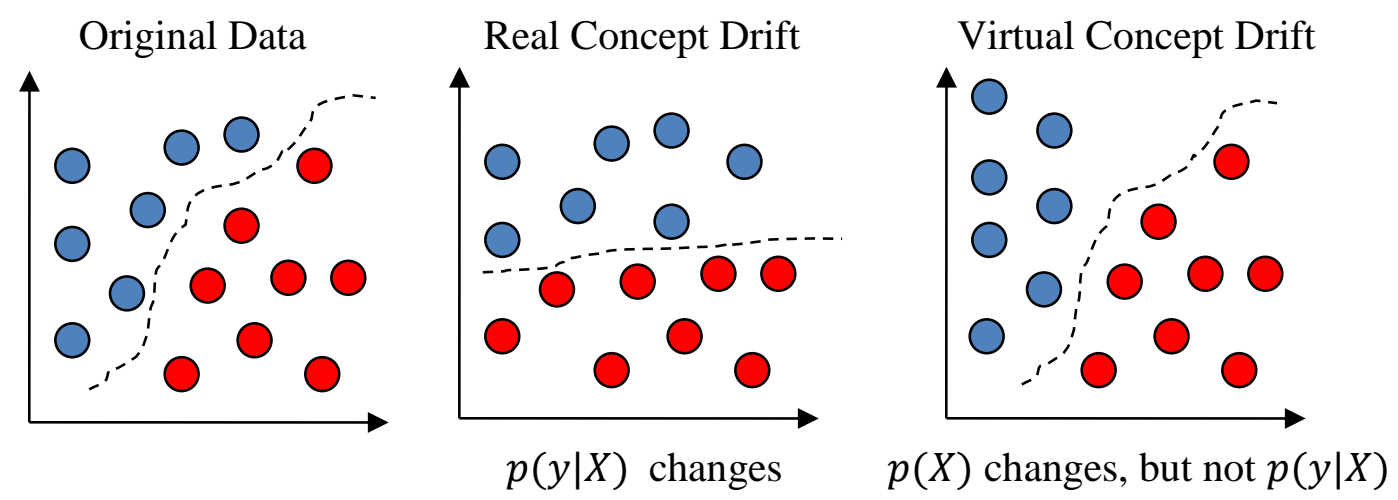

Figure 2. These graphs show types of drifts. The circles represent instances, different colors representing different classes, and dotted lines representing class boundaries (adapted from Gama et al., 2014).

In Figure 2, with real concept drift the boundary between the class labels changes but the input data does not change. When virtual concept drift occurs, the input data changes but the boundary between class labels does not change. Most of the concept drift research refers to real concept drift. The work in this study will focus on real concept drift and future references to concept drift will imply real concept drift. 


\section{Highlights in Concept Drift Research}

The first work on concept drift was by Schlimmer and Granger (1986). Their approach used a set of weighted symbolic characteristics to describe concepts. Learning systems were then used to adjust these weights and create new characterizations to describe the concepts. This technique, known as STAGGER, served as the basis for later studies. Klenner and Hahn (1994) used frame representation to handle gradual concept drift. Widmer and Kubat (1996) created a system, called FLORA, that dynamically adjusted a window of time to refine positive, negative, and potential rules in order to track concept drift. Widmer (1997) used naïve Bayes and meta-learning to handle reoccurring concepts. Klinkenberg and Joachims (2000) studied the use of a support vector machine to size windows for concept drift. A concept-adapting Very Fast Decision Tree (CVFDT) learner (Hulten, Spencer, \& Domingos, 2001) added concept drift to the work on VFDT (Domingos \& Hulten, 2000). Chandola, Banerjee and Kumar (2009) explored the challenge of confusing concept drift with an outlier or noise.

\section{Ensemble Methods with Concept Drift}

Ensemble methods have proven to perform well with concept drift and researchers have created online versions of popular ensemble methods, such as online AdaBoost (Fan, Stolfo, \& Zhang, 1999). As mentioned earlier, Fern and Givan (2003) provided evidence that ensembles of small trees gave greater classification accuracy than a single tree. Wang et al. (2003) showed that a classifier ensemble can improve on a single classifier when concept drift is present. Gao, Fan, and Han (2007) suggested that unweighted ensembles may be beneficial in the presence of concept drift. Blum (1997) used an incremental approach to concept drift. Here experts were created from pairs of attributes and predictions were made by using majority vote (Littlestone \& Warmuth, 
1994) from the results of all possible pairs. After the system received the correct class label, experts that predicted incorrectly had their weights cut in half. Other research conducted using ensemble methods to discover concept drift include work by Street and Kim (2001). Their Streaming Ensemble Algorithm (SEA) approach used a fixed-size collection of classifiers built from training examples. When new examples appeared a new classifier was created and the new classifier was added to the ensemble if room was available. If room was not available, a poorer performing classifier was removed to make room for the new classifier. Predictions were made by majority vote. Scholz and Klinkenberg (2006) created two ensembles and then chose the best for later processing.

\section{Concept Drift Detection}

There are two major classifications of strategies for using ensemble methods to detect concept drift according to the research of Minku and Yao (2012). One ensemble approach to check for concept drift is to include a mechanism to detect drifts explicitly. Here a measure is typically used to determine if a concept drift has occurred. If a concept drift is detected, a new online classifier is created and all classifiers in the ensemble are re-trained. This approach tends to react quickly to concept drift if it is found early. On the negative side, the explicit approach can sometimes detect a drift where a drift has not occurred. One example of this explicit technique is the work by Baena-García, Del Campo-Ávila, Fidalgo, and Bifet (2006), called the Early Drift Detection Method (EDDM). With this approach it is assumed that when a stable concept is in the process of being learned, the difference in time between consecutive errors will be larger. A noticeable drop in this difference is considered a concept drift. A new classifier system is generated at this point. 
The second approach handles concept drift implicitly. Here it is common to attach weights to the classifier of each ensemble. These weights are based on accuracy and provide for addition and deletion of new classifiers. Representative of this approach is the work of Kolter and Maloof (2007). Their Dynamic Weighted Majority (DWM) ensemble set up a group of weighted classifiers. Classifiers were added and deleted based on the effectiveness of those classifiers. Classifier weight was reduced if a mistake was made. In addition, the experts which performed poorly were deleted if their weights fell below a predefined threshold. The downside of this approach is that time is required for the classifier weights to represent the new concept.

\section{EDDM}

Concept drift can occur abruptly or gradually. The method used by Gama, Medas, Castillo, and Rodrigues (2004) detected concept drift by counting the number of errors found in examples. This method worked well for abrupt concept drift but did not achieve good performance if the drift was gradual. Gradual concept changes are more difficult to detect, partly because of the need for increased resources to store additional examples. As stated earlier, the EDDM algorithm (Baena-García et al., 2006) identified concept drift by keeping track of the number of time steps between classification errors. EDDM used a warning threshold and a concept drift threshold to determine when a new concept was present. If the warning threshold was reached, examples were saved in preparation for new concept learning. If the concept drift threshold was met, the old learning model was reset and a new learning model was created using the examples saved after the warning threshold was reached. EDDM performed well on both abrupt and gradual drifts when compared to similar drift detection methods. Following are the calculations used with warning level $(\alpha)$ and drift level $(\beta)$ : 


$$
\begin{array}{ll}
\left(p_{i}^{\prime}+2 s_{i}^{\prime}\right) /\left(p_{\max }^{\prime}+2 s_{\max }^{\prime}\right)<\alpha & \text { (for the warning level) } \\
\left(p_{i}^{\prime}+2 s_{i}^{\prime}\right) /\left(p_{\max }^{\prime}+2 s_{\max }^{\prime}\right)<\beta & \text { (for the drift level) }
\end{array}
$$

Here $p_{i}^{\prime}$ is the average difference in time steps between errors in classification and $s_{i}^{\prime}$ is the standard deviation of this average. Also, $p_{\max }^{\prime}$ and $s_{\max }^{\prime}$ hold the highest values of $p_{i}^{\prime}$ and $s_{i}^{\prime}$, respectively. Calculations are done after 30 errors have occurred. The number 30 was chosen so a distribution of error differences can be compared to other distributions. The denominator $p_{\max }^{\prime}+2 s_{\max }^{\prime}$ represents $95 \%$ of the distribution. Figure 3 shows how the thresholds $\alpha$ and $\beta$ are used in EDDM.

Figure 3. Concept Drift Detection with EDDM

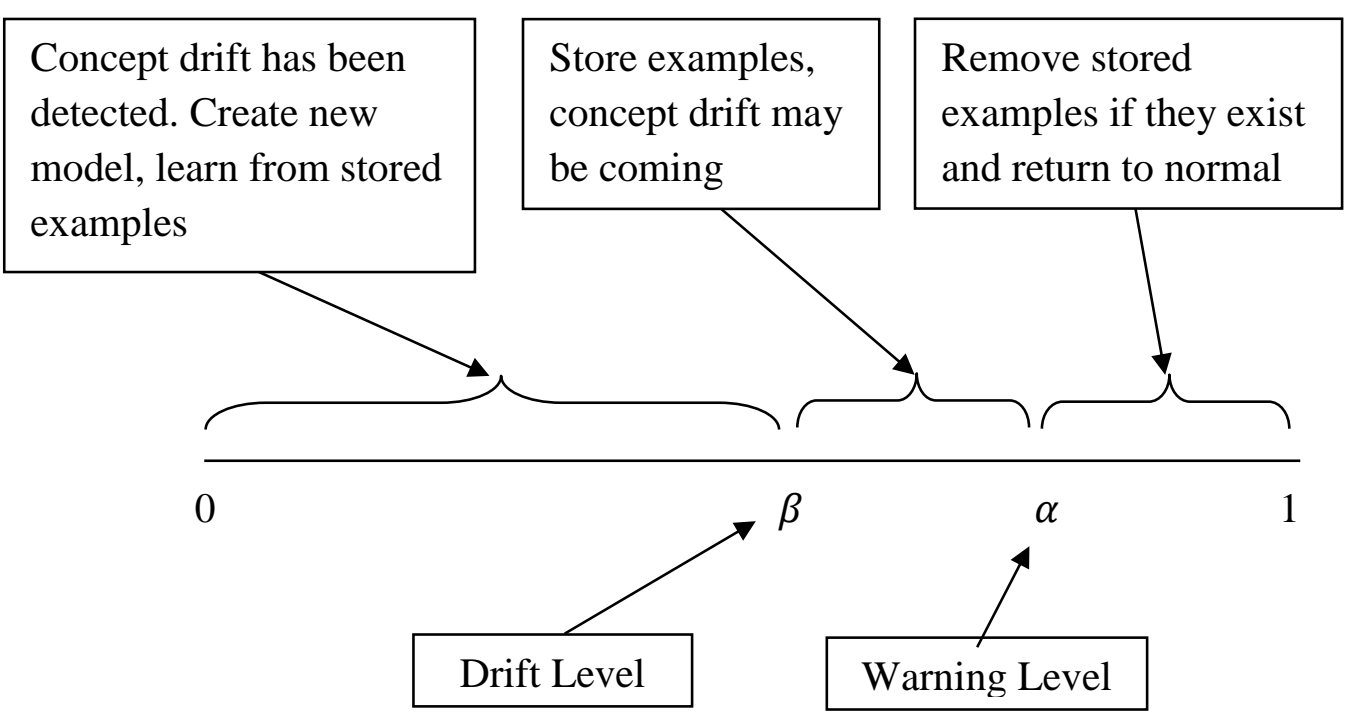

Figure 3. This is a description of the relationship between $\alpha$ and $\beta$ in the EDDM algorithm.

As can be seen in Figure 3, the system runs normally from a value of $\alpha$ and above. Examples are stored when the threshold is between $\alpha$ and $\beta$. Results below $\beta$ 
signal that a concept drift has been detected. At this point the current model is reset and a new model learns using the stored examples.

\section{DWM}

As mentioned earlier, concept drift can also use implicit concept drift detection.

Algorithm 2 shows the DWM algorithm of Kolter and Maloof (2007).

\section{Algorithm 2. An overview of the DWM algorithm}

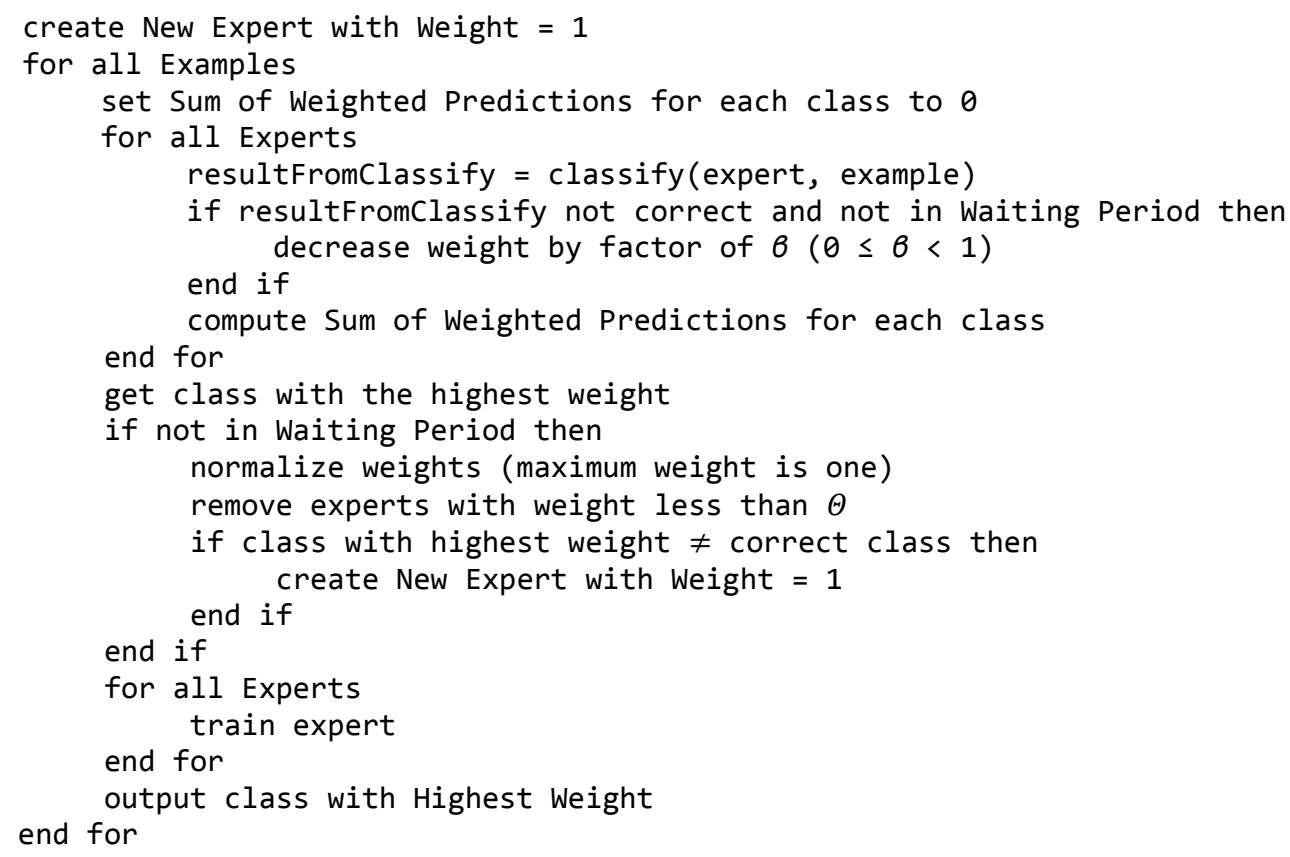

Algorithm 2 begins with the creation of a single ensemble with a weight of one

(line 1). The current example is then given to the expert for classification (line 5). If the classification is not correct, the expert's weight is decreased by a factor of $\beta$ (lines 6 and 7). A weighted sum is then computed for each class (line 9). The class with the highest weight (line 11) is identified as the global prediction. Weights of ensembles are 
normalized so they can be compared (line 13) and poorly performing experts are removed (line 14). If the global prediction (class with the highest weight) is incorrect, a new expert is created (lines 15 and 16). DWM includes a parameter for a waiting period. During the waiting period, the weights of experts are not changed and experts are not added and deleted (lines 6 and 12).

\section{Diversity with Ensembles}

The use of diversity in base classifiers of ensembles has been studied. The ensemble techniques of bagging and boosting utilized a diverse set of classifiers. Dietterich (2000) tested the randomization, bagging, and boosting ensemble techniques and found that when classification noise was present, bagging out-performed boosting and randomization in most cases. In the presence of noise, bagging appeared to be able to use the classification noise to its advantage. The study by Breiman (2001) found that random forests with lower error tended to have lower base classifier correlation and higher classification accuracy. Guerra-Salcedo and Whitley (1999) used a generic algorithm (GA) to create the components of an ensemble. Their results revealed that diversity created by the GA improved on that of random ensembles. The research of Kuncheva and Whitaker (2004) identified that diversity in ensembles was important but that it was difficult to measure diversity. The addition of ensembles with high and low diversity levels was explored by Minku and Yao (2012) and their results improved on work from similar studies.

Minku and Yao (2012) found that a range of diversity levels in ensembles gave the maximum prequential accuracy. 
Prequential accuracy was described by Dawid and Vovk (1999) and is the average accuracy of predicted examples, prior to the examples being learned and is computed by:

$$
\operatorname{acc}(t)= \begin{cases}\operatorname{acc}_{e x}(t), & \text { if } t=f, \\ \operatorname{acc}(t-1)+\frac{\operatorname{acc}_{e x}(t)-\operatorname{acc}(t-1)}{t-f+1}, & \text { otherwise, }\end{cases}
$$

In this equation $a c c_{e x}$ is 0 when the prediction of the current training example $e x$ is incorrect and 1 if the training example is correct, $f$ is the first time step used when calculating the data, and $t$ is the time step. Minku \& Yao (2012) studied the ensembles used both before and after the start of concept drift. As part of this analysis, prequential accuracy was reset when the drift began $(f \in\{1, N+1\})$. Here $N$ represents the number of time steps before the concept drift began.

Diversity levels were controlled in DDD using a modified version of the algorithm from Minku, White, and Yao (2010). Their work was influenced by the online bagging technique of Oza and Russell (2001). This technique is shown in Algorithm 3.

\section{Algorithm 3. Modified Online Bagging}

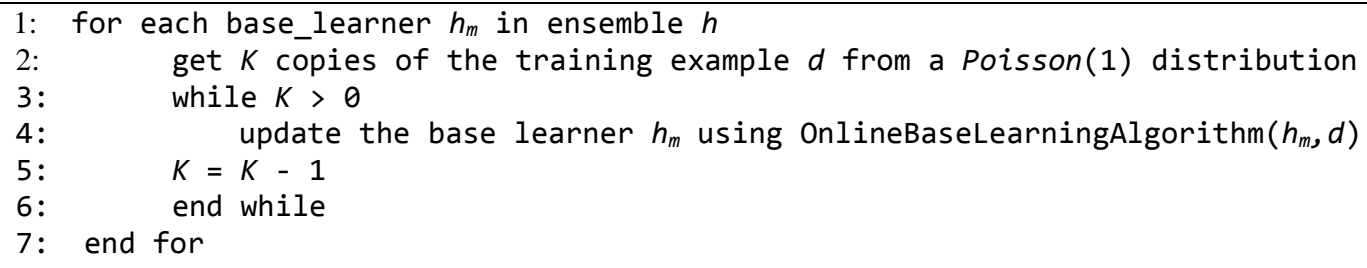

Algorithm 3 uses the idea that as the number of training examples gets large, each base learner holds $K$ copies of the original training example (lines 3-6). It turns out that the distribution of $K$ looks like a Poisson(1) distribution, so as new examples are obtained, the 
number of times each base learner sees the example is taken from a Poisson(1)

distribution (line 2). The calculation of $K$ can be changed to Poisson $(\lambda)$ to create diversity in ensembles. A higher $\lambda$ gives lower diversity and a lower $\lambda$ gives higher diversity.

To measure diversity, Minku and Yao (2012) followed the recommendation of Kuncheva and Whitaker (2003) and used Yule's Q statistic (1900) which follows:

$$
Q_{i, k}=\frac{N^{11} N^{00}-N^{01} N^{10}}{N^{11} N^{00}+N^{01} N^{10}}
$$

Given two classifiers $D_{i}$ and $D_{k}, N^{a, b}$ was the number of training examples where the classification of $D_{i}$ is $a$ and the classification of $D_{k}$ was $b$. Here 1 was a correct classification and 0 was an incorrect classification. Q values were in the range $[-1,1]$ and tended to be positive the more classifiers agreed on a classification. In the study by Minku and Yao (2012), the Q statistic was averaged over every pair of classifiers to provide a metric for diversity. A high average Q statistic represented low diversity and a low average Q statistic represented high diversity.

\section{DDD Algorithm}

In the DDD algorithm of Minku and Yao (2012), discussed in chapter 1, two ensembles were used before concept drift was detected, one with low diversity and the other with high diversity. If concept drift was detected, two additional ensembles were created. One of these had low diversity and the other had high diversity. These four ensembles remained in memory until either of the following conditions occurred: The new low diversity ensemble had better accuracy than either of the two old ensembles or the old high diversity ensemble had better accuracy than the new low and old low diversity ensembles. It is not known if placing a maximum limit on the number of time steps these four ensembles were in memory would provide comparable accuracy. If this 
maximum limit on time steps does provide comparable accuracy, it would be an improvement on the current method in two ways. First, memory usage should decrease because the additional ensembles would need to be in memory a shorter time. Second, the time the DDD algorithm takes to run should decrease because the two additional ensembles would not have to be maintained as long. Since this extra maintenance would not be required, less processing would be needed and run time should go down.

\section{False Positives in Concept Drift}

It is important for a learning system to be accurate in the presence of concept drift, and it is also important to keep concept drift from being detected when it is not present. Minku and Yao (2012) addressed this issue by using an additional parameter, named $W$, as a multiplier on the weight of the old low diversity ensemble. Increased values of $W$ allowed DDD to detect concept drift false alarms more easily, but accuracy was sacrificed when real concept drift occurred. A lower value for $W$ made DDD less able to detect false alarms, but accuracy improved with this lower setting when real concept drift arose.

\section{Comparison of EDDM, DWM, and DDD}

Minku and Yao (2012) compared the DDD, EDDM, and DWM algorithms. Different diversity levels were used with DDD to test the impact of diversity levels on accuracy. In the first concept, DDD and EDDM were similar if false positive concept drift did not exist. When there was false positive concept drift, DDD was more accurate than EDDM because EDDM resets its accuracies when a false positive concept drift occurs. In this case the knowledge of the current concept is lost. DDD, on the other hand, 
increases the old ensemble weights so that false positive concept drift is less likely in the future.

After concept drift has been detected, DDD performed better than EDDM on most kinds of drifts, because of its ability to learn from the old ensembles. DDD also gave higher accuracy than DWM, whether concept drift was present or not.

\section{Performance Evaluation}

When evaluating machine-learning techniques, performance evaluation metrics are needed as well as ways to train and test the machine-learning techniques (Gama et al., 2014). When memory usage is part of the study, RAM-hours have been used as a performance metric (Bifet, Holmes, Pfahringer, \& Frank, 2010). RAM-hours are computed from the rental cost in a cloud-computing environment. The use of one gigabyte of RAM for one hour is one RAM-hour. To compare the accuracy of an intelligent classifier to a random classifier, the kappa-statistic is defined as:

$$
\frac{a-a_{r}}{1-a_{r}}
$$

where the accuracy of the intelligent classifier is $a$ and the accuracy of a random classifier is $a_{r}$. A kappa-statistic closer to 1 indicates the intelligent classifier is closer to a perfect classifier. A kappa-statistic of 0 means the intelligent classifier is no better than a random classifier. The kappa-statistic has been found to be a good alternative to Receiver Operating Characteristic (ROC) curves when streaming data is being evaluated and classifiers are being compared. Bifet, Holmes, and Pfahringer (2010) used the kappastatistic to compare leverage bagging and online bagging. Minku and Yao (2012) used $t$ tests to compare the accuracy of various concept drift techniques. These $t$-tests were done right before concept drift, right after concept drift, a medium time after concept drift, and a long time after concept drift. A common way to measure performance in concept drift 
algorithms is by graphing prequential accuracy (defined in Chapter 1) over time steps. Concept drift algorithms that use this approach include: DDD (Minku \& Yao, 2012), EDDM (Baena-García et al., 2006), and DWM (Kolter and Maloof, 2007). It is also common to add one \pm standard deviation to the average prequential accuracy. Minku and Yao (2012) also graphed the change in weights on the new low, old low, and old high diversity ensembles over time steps.

While supervised learning systems typically use cross-validation to estimate performance with static data, this approach does not translate well with data that contains concept drift. According to Gama et al. (2014), cross-validation could mix the data in such a way that the temporal order of the data would be lost. Three techniques commonly used to determine the data for training and testing are: holdout, prequential, and controlled permutations. With holdout evaluation, a subset of training and testing data is used for testing. The holdout set maintains the same concepts as does the training and testing sets, only on a smaller amount of data. Prequential evaluation (defined in Chapter 1) allows individual examples to be tested before they are used in the training phase. A holdout set is not required for this technique. Controlled permutations (Žliobaitè, I., 2011) use randomized copies as in cross-validation. The difference is that controlled permutations attempt to keep data in its original position.

\section{Summary}

This chapter contains a review of the literature in concept drift research. From this review there is a high likelihood that increases in data stream data will continue to provide situations where concepts will change over time. It is also clear that there is a strong need for improved algorithms to handle concept drift effectively. In addition, it was shown that an ensemble of classifiers provides better classification accuracy than a 
single classifier. Research using diversity in ensembles (Minku \& Yao, 2012) was shown to be a promising approach to improved classification. Also, adding additional ensembles appears to provide greater accuracy. There is a tradeoff, however, because the additional ensembles require more time and space resources. A study of the impact on accuracy of placing a maximum on the number of time steps that four ensembles can be in memory appears to be a valid path for continued research. 


\section{Chapter 3}

\section{Methodology}

\section{Overview of Methodology}

As described in the problem statement, this dissertation investigated how prequential accuracy in the DDD algorithm is affected by the placement of a maximum on the number of time steps four ensembles are stored in memory. For discussion purposes this maximum was called the maximum time step parameter and was defined as MTS. A secondary portion of my work examined the impact of changes in the $W$ parameter with the maximum limit time step parameter included. The $W$ parameter was used in the DDD algorithm as a weight on the old low diversity ensemble to detect false concept drift. This section begins with a broad overview of my dissertation approach. A breakdown of these steps follows. After this, preliminary work done is mentioned and knowledge gained from this work is described. Finally, the details of how results were analyzed is given and a listing of resources used is shown. Here is the broad overview of the method I used in my dissertation:

1. Created an additional parameter to the DDD algorithm that put a maximum limit on the number of time steps four ensembles were in memory when concept drift was detected (the MTS parameter). This parameter took on a range of values to test its impact on prequential accuracy.

2. Tested the DDD algorithm with a range of values in the MTS parameter on the artificial datasets used by Minku and Yao (2012). Low, medium, and high speed concept drift data were used along with low, medium, and high severity concept drift data.

3. Tested a range of values in the MTS parameter using some of the real world datasets found in Minku and Yao (2012), Gama, Rocha, and Medas (2003), and Oza and Russell (2001).

4. Compared the DDD algorithm using the most promising MTS parameter to the DDD algorithm without the MTS parameter and to a version of the DDD algorithm that had its learning reset after concept drift was encountered. 
5. Modified the values for the $W$ parameter in the DDD algorithm. The $W$ parameter was used in the DDD algorithm as a weight on the old low diversity ensemble to help keep the algorithm from reacting to what appears to be a concept drift but is not. The impact of changes in $W$ related to settings in the MTS parameter were explored and were compared to changes in the $W$ parameter using the DDD algorithm without the MTS.

\section{Maximum Time Step Parameter}

An additional parameter to the DDD algorithm was created. This parameter placed a maximum on the number of time steps that four ensembles were kept in memory when concept drift was detected. The added code created a condition to return the state of the DDD algorithm to before-drift-mode when the maximum time step was reached. At this point the number of ensembles reduced from four to two.

\section{Testing with Artificial Datasets}

Analysis of concept drift with real world datasets is difficult. With real world datasets, the location of and presence of concept drift is not always known. That is why it is common in concept drift research to use artificial data that can be controlled. The artificial datasets used were the same as those used in Minku and Yao (2012). These datasets were circle, sine moving vertically, sine moving horizontally, line, plane, and Boolean. The Boolean dataset was derived from the original STAGGER problem (Schlimmer \& Granger, 1986). The attributes color, shape, and size were used with the Boolean dataset to determine if the object was in class 1 or class 0 .

Various speed and severity values were used with this artificial data. As mentioned earlier, speed is the inverse of drifting time, which is the number of time steps needed for the new concept to take the place of the old concept. The degree of dominance of the new and old concepts was defined by Narasimhamurty and Kuncheva (2007). 
Here $V_{n}(t)$ is the probability the new concept will be presented to the system and $V o(t)$ is the probability the old concept will be presented to the system and are defined as:

$$
v_{n}(t)=\frac{t-N}{\text { drifting_time }}, \quad N<t \leq N+\text { drifting_time }
$$

and

$$
v_{0}(t)=1-v_{n}(t), \quad N<t \leq N+\text { drifting_time }
$$

In these equations, $t$ is the current time step, $N$ is the number of steps before the concept drift, and drifting_time represents the time steps required for the new concept to completely replace the old concept. Each of these artificial datasets contained one concept drift and contained $2 N$ examples. The old concept $v_{0}(t)$ was used for the first $N$ examples $(1 \leq t \leq N)$. The next drifting_time examples used the probabilities of $v_{0}(t)$ and $v_{n}(t)$ to determine whether to use the old or new concept $(N<t \leq N+$ drifting_time $)$. After this time, the remaining examples $(N+$ drifting_time $<t \leq 2 N)$ were generated by the new concept $v_{n}(t)$. Speeds for the artificial datasets used drifting_time values of 1 , $0.25 N$, and $0.50 N$ time steps as was done in Minku and Yao (2012). This allowed for the creation of fast, medium, and slow speeds.

As stated earlier, severity is defined as the percentage of input data that has its target class modified when the drift is complete. Table 3 shows the severity changes for all the artificial datasets to be used for this study. 
Table 3. Severity for Artificial Datasets

\begin{tabular}{|c|c|c|c|c|}
\hline Problem & Equation & $\begin{array}{c}\text { Fixed } \\
\text { Values }\end{array}$ & $\begin{array}{c}\text { Before } \rightarrow \text { After } \\
\text { Drift }\end{array}$ & Severity \\
\hline Circle & $\begin{array}{c}(x-a)^{2}+(y-b)^{2} \leq \\
r^{2}\end{array}$ & $\begin{array}{l}a=.5 \\
b=.5\end{array}$ & $\begin{array}{l}r=.2 \rightarrow .3 \\
r=.2 \rightarrow .4 \\
r=.2 \rightarrow .5\end{array}$ & $\begin{array}{ll}16 \% & \text { Low } \\
38 \% & \text { Medium } \\
66 \% & \text { High }\end{array}$ \\
\hline SineV & $y \leq a \sin (b x+c)+d$ & $\begin{array}{l}a=1 \\
b=1 \\
c=0\end{array}$ & $\begin{array}{l}d=-2 \rightarrow 1 \\
d=-5 \rightarrow 4 \\
d=-8 \rightarrow 7\end{array}$ & $\begin{array}{ll}15 \% & \text { Low } \\
45 \% & \text { Medium } \\
75 \% & \text { High }\end{array}$ \\
\hline SineH & $y \leq a \sin (b x+c)+d$ & $\begin{array}{l}a=5 \\
b=5 \\
c=1\end{array}$ & $\begin{array}{l}c=0 \rightarrow-\pi / 4 \\
c=0 \rightarrow-\pi / 2 \\
c=0 \rightarrow-\pi\end{array}$ & $\begin{array}{ll}36 \% & \text { Low } \\
57 \% & \text { Medium } \\
80 \% & \text { High }\end{array}$ \\
\hline Line & $y \leq-a_{0}+a_{1} x_{1}$ & $a_{1}=.1$ & $\begin{array}{l}a_{0}=-.4 \rightarrow-.55 \\
a_{0}=-.25 \rightarrow-.7 \\
a_{0}=-.1 \rightarrow-.8\end{array}$ & $\begin{array}{ll}15 \% & \text { Low } \\
45 \% & \text { Medium } \\
70 \% & \text { High }\end{array}$ \\
\hline Plane & $y \leq-a_{0}+a_{1} x_{1}+a_{2} x_{2}$ & $\begin{array}{l}a_{1}=.1 \\
a_{2}=.2\end{array}$ & $\begin{array}{l}a_{0}=-2 \rightarrow-2.7 \\
a_{0}=-1 \rightarrow-3.2 \\
a_{0}=-.7 \rightarrow-4.4\end{array}$ & $\begin{array}{ll}14 \% & \text { Low } \\
44 \% & \text { Medium } \\
74 \% & \text { High }\end{array}$ \\
\hline Boolean & $\begin{array}{l}\text { (color eq } q_{1} a \\
\text { op1 } \\
\left.\text { shape eq } q_{2} b\right) \\
\text { op2 } \\
\text { size eq } q_{3} c\end{array}$ & $\begin{array}{c}c= \\
S \vee M \vee L \\
o p_{2} \wedge \\
e q_{1,2,3}=\end{array}$ & $\begin{array}{l}\begin{array}{l}a=R, o p_{1} \wedge \\
b=R \rightarrow R \vee T\end{array} \\
\begin{array}{l}a=R, b=R, \\
o p_{1} \wedge \rightarrow \vee\end{array} \\
\begin{array}{l}a=R \rightarrow R \vee G, \\
b=R \rightarrow R \vee T \\
o p_{1} \wedge \rightarrow \vee\end{array}\end{array}$ & $\begin{array}{l}\text { 11\% Low } \\
\text { 44\% Medium } \\
\text { 67\% High }\end{array}$ \\
\hline
\end{tabular}

Table 3 uses $a, b, c, d, r, a_{i}, e q$, and $o p$ to define values for equations that represent different concepts. In the SineV equation, for example, $15 \%$ of the input data had its target class modified when the variable $d$ changed from -2 to 1 in the SineV formula. This represents low severity. Also, $45 \%$ of the input data had its target class modified when $d$ changed from -5 to 4 in the SineV formula, indicating medium severity. The severity used with these datasets was low, medium and high. Having three settings for speed and three settings for severity allowed for the creation of nine comparisons for each of the artificial datasets. The artificial datasets were tested with Incremental Tree Inducer (ITI) lossless decision trees as base learners (Utgoff, Berkman, \& Clouse, 1997). The ensemble size was 25 ITI lossless decision trees and each dataset ran 30 times to 
produce the average prequential accuracy. The test values for the MTS parameter were $31,50,75$, and 100 . The selection of 31 was used because 31 is the minimum possible for the DDD, since the DDD algorithm requires the four ensembles to be in memory at least 30 time steps. The other three $M T S$ values $(50,75,100)$, where chosen because they are the next three multiples of 25 after the minimum value of 31 . The $\lambda_{l}$ low diversity parameter was set to 1.0 as was done in Minku and Yao (2012). For the $\lambda_{h}$ high diversity ensembles, this value was 0.05 for circle, sineH, and plane. SineV was set to 0.005 and Boolean was 0.1 for $\lambda_{h}$ high diversity ensembles. These values for the high diversity ensemble setting $\lambda_{h}$ were the same as those used by Minku and Yao (2012) that showed good results. Each artificial dataset contained $2 N$ examples; one example represented one time step. Circle, sineV, sineH, and line had an $N$ value of 1000 . The $N$ value for both plane and Boolean was 500. Table 4 shows additional settings for the artificial datasets. 
Table 4. Settings for Artificial Datasets

\begin{tabular}{|c|c|c|c|c|c|}
\hline Dataset & $\begin{array}{c}\text { Training } \\
\text { File Size }\end{array}$ & $\begin{array}{c}\text { Testing } \\
\text { File Size }\end{array}$ & Range of X & Range of Y & $\begin{array}{c}\text { High } \\
\text { Diversity } \\
\lambda_{h}\end{array}$ \\
\hline Circle & 2000 & 500 & {$[0,1]$} & {$[0,1]$} & 0.05 \\
\hline SineV & 2000 & 500 & {$[0,10]$} & {$[-10,10]$} & 0.005 \\
\hline SineH & 2000 & 500 & {$[0,4 \pi]$} & {$[0,10]$} & 0.05 \\
\hline Line & 2000 & 500 & {$[0,1]$} & {$[0,1]$} & 0.005 \\
\hline Plane & 1000 & 200 & {$[0,1]$} & {$[0,5]$} & 0.05 \\
\hline Boolean & 1000 & 200 & $\begin{array}{c}(\mathrm{R}, \mathrm{G}, \mathrm{B}) \text { Color } \\
(\mathrm{T}, \mathrm{R}, \mathrm{C}) \text { Shape } \\
(\mathrm{S}, \mathrm{M}, \mathrm{L}) \text { Size }\end{array}$ & {$[0,1]$} & 0.1 \\
\hline
\end{tabular}

\section{Testing with Real World Data}

To be beneficial, new algorithms for concept drift detection need to work with real world data. Some of the same real world datasets used by Minku and Yao (2012) were used in this research. The first of these real world datasets, described by Harries (1999), is an electricity dataset from the Australian New South Wales Electricity Market. The dataset contained 45,312 examples made up of the input attributes: time stamp, day of week, and two electricity demand values. The target class is the change in the price of electricity. Price is affected by supply and demand. During the time period of this dataset (May 1996 to December 1998), an expansion of the electricity area caused a decrease in electricity price. This decrease in price represents concept drift. The second real world dataset was the KDD Cup 1999 network intrusion data (The UCI KDD Archive, 1999). This dataset contains 494,090 examples. The 41 input attributes of this dataset includes 
connection length, protocol type, and destination network service. The target class is connection status (attack or normal). This dataset simulates a military environment where attacks were so common that attack occurred more frequently than did normal. The third real world dataset was the Forest Covertype dataset (Asuncion \& Newman, 2007). This dataset has been used by other concept drift researchers (Gama, Rocha, \& Medas, 2003; Oza \& Russell, 2001). Forest Covertype is made of 30 x 30 meter cells from the US Forest Service. The dataset contains 581,012 examples with 54 attributes. The class is the type of forest suggested by the attributes. ITI decision trees were used as base learners with the real world datasets. The ensemble size for the ITI decision trees was 25 . As with the artificial data, each real world dataset ran 30 times to produce the average prequential accuracy and the values used to test the MTS parameter were $31,50,75$, and 100 . See the previous section for the details that explain the choice of MTS values. Since accuracy and not speed was the focus of this dissertation, a subset of the full real world datasets was used. With all three real world datasets, the training file size was 2000 and the testing file size was 500. These sizes were chosen because they matched the size of four of the six artificial datasets. All three real world datasets used training files created from the first $80 \%$ of the original data. The testing files were created from the remaining $20 \%$ of the original data. As with the artificial datasets, the low diversity ensemble setting $\lambda_{l}$ was 1.0 for all real world datasets. The real world data high diversity ensembles had the $\lambda_{h}$ setting of 0.005 , the same as used by Minku and Yao (2012) for Electricity and the KDD Cup 1999 datasets. The settings for the real world datasets are found in Table 5. 
Table 5. Settings for Real World Datasets

\begin{tabular}{|c|c|c|c|c|}
\hline Dataset & $\begin{array}{c}\text { Training File } \\
\text { Size }^{\mathbf{a}}\end{array}$ & $\begin{array}{c}\text { Testing } \\
\text { File Size }^{\mathbf{b}}\end{array}$ & Range of X & Range of Y \\
\hline Electricity & 2000 & 500 & $\begin{array}{c}\text { Time Stamp, } \\
\text { Day of Week, } \\
\text { Two elec. } \\
\text { demand values }\end{array}$ & [Up, Down] \\
\hline KDD Cup 1999 & 2000 & 500 & 41 Attributes & [Attack ${ }^{\mathrm{c}}$, Normal] \\
\hline Forest CoverType & 2000 & 500 & 54 Attributes & {$[1,2,3,4,5,6,7]$} \\
\hline
\end{tabular}

Notes. ${ }^{\text {a }}$ Training File was created from the first $80 \%$ of the original dataset

${ }^{b}$ Testing File was created from the last $20 \%$ of the original dataset

${ }^{\mathrm{c}}$ Attack in this table represents anything other than normal

\section{Comparison with other Concept Drift Algorithms}

The new version of the DDD algorithm with the MTS parameter was tested against the original version of the DDD algorithm and against a version of the DDD algorithm where the learning system was reset when a concept drift was encountered. The value of the MTS parameter that gave the best results was used for this comparison.

\section{Testing for False Positive Concept Drift}

The $W$ parameter was used in the DDD algorithm as a weight on the old low diversity ensemble to help keep the algorithm from reacting to what appears to be concept drift but is not. The impact of changes in $W$ related to settings in the MTS parameter was explored. Following Minku and Yao (2012), additional testing was done by forcing false concept drift at specific times with the artificial data and the real world data. It was expected that higher values of $W(W=3)$ would improve accuracy in the presence of false 
alarms, but would not be as accurate when real concept drift occurred. It was expected that lower values of $W(W=1)$ should make the new DDD algorithm less robust when false alarms are in the data, but provide greater accuracy when real concept drift occurred. Changes in the $W$ parameter (using $W=1, W=2$, and $W=3$ ) were compared to the modified DDD algorithm using the best setting found for the MTS parameter. False alarms were created during the old concept and the new concept with the artificial datasets. Changes in the $W$ parameter were also conducted with the real world datasets. The best MTS parameter found was used with different $W$ parameter values $(W=1, W=2$, and $W=3$ ) to see how accuracy was affected when false positive concept drift occurred during the old and new concepts.

\section{Preliminary Work}

Preliminary work was done on this research. The following steps were taken:

- A counter was added to compute the number of time steps the DDD algorithm used four ensembles to learn the new concept.

- Tests were conducted with the artificial datasets of Minku and Yao (2012) using various severities and speeds. Comparisons were made to the results found by these researchers.

- Tests were conducted with the artificial datasets of Minku and Yao (2012). It was noted that the real world datasets contained a much higher number of examples than did the artificial datasets.

From this work it was found that:

- The results obtained were comparable to those found by Minku and Yao (2012). Since randomization was involved, exact results were not found, but the average accuracies obtained by averaging 30 runs were close to those of these researchers and the standard deviations of the 30 runs were comparable as well. 
- While the accuracies of the 30 runs on the artificial data did not have a large standard deviation, the number of steps the DDD algorithm using four ensembles varied greatly even when the same datasets were employed. In fact, the only difference in input between the 30 runs was the difference in the random seed sent to the Poisson distribution to create diversity in ensembles. For testing, I used the number 1 for test \#1, number 2 for test \#2, and so on for the 30 runs. The random seed setting was monitored to see if some pattern existed among different datasets.

- The amount of time required to run one dataset (Circle) for one severity and speed ranged from 3 to 10 minutes, depending on the speed of the computer used. Even at 3 minutes per run, the time needed to run all artificial datasets under three methods (DDD using the MTS parameter, original DDD, and DDD resetting ensembles after concept drift is detected), nine severity/speed settings, and four MTS settings was prohibitive. Running the real world datasets would be time intensive as well. To help decrease overall running time, multiple computers were used in this study.

Since the process of testing was time consuming, programs to automate the testing process were written (in Python). The first of these programs was CreateBatchFile.py. This program allows a user to enter all parameters required to run the ITI version of DDD. CreateBatchFile.py then created 30 lines of commands that were used to run the specified artificial dataset 30 times. The process was repeated for each of the six artificial datasets. Table 6 shows the parameters entered for the CreateBatchFile.py program. Also included in Table 6 are two additional parameters used in this study, reset-after-drift and MTS. The reset-after-drift parameter allowed accuracies to be reset and also kept the two extra ensembles from being created when concept drift was detected. The MTS parameter placed a maximum on the number of time steps four ensembles were in memory after concept drift detection. 
Table 6. Parameters for CreateBatchFile.py

\begin{tabular}{|c|c|}
\hline Parameter & Description \\
\hline program_name & $\begin{array}{l}\text { The original DDD algorithm, the new DDD } \\
\text { algorithm with the } M T S \text {, or the DDD algorithm } \\
\text { that resets accuracies }\end{array}$ \\
\hline training_file & Name of training file (.csv or .txt) \\
\hline num_train_items & Number of examples in the training file \\
\hline test_file & Name of the test file \\
\hline num_test_items & Number of rows in the test file (.csv or .txt) \\
\hline names_file & Format of attributes and classes \\
\hline base_model_num & $\begin{array}{l}\text { Number of classifiers in each ensemble ( } 25 \text { for } \\
\text { the ITI decision trees) }\end{array}$ \\
\hline rand_seed & $\begin{array}{l}\text { Random seed for the Poisson distribution (for } \\
\text { ensemble diversity) }\end{array}$ \\
\hline trans_drift_interval & $\begin{array}{l}\text { Tells the interval used for concept drift in the } \\
\text { training file }\end{array}$ \\
\hline test_drift_interval & $\begin{array}{l}\text { Tells where to move to in the test file after } \\
\text { concept drift occurs in the training file }\end{array}$ \\
\hline poisson_parameter_low_div & $\begin{array}{l}\text { Poisson value for low diversity ensemble } \\
\text { (normally 1.0) }\end{array}$ \\
\hline poisson_parameter_high_div & $\begin{array}{l}\text { Poisson value for high diversity ensemble (varies } \\
\text { by dataset) }\end{array}$ \\
\hline
\end{tabular}




\begin{tabular}{|c|c|}
\hline Parameter & Description \\
\hline waiting_time & $\begin{array}{l}\text { Minimum time steps to maintain four ensembles } \\
\text { (normally } 30 \text { ) }\end{array}$ \\
\hline outcontrol_level & $\begin{array}{l}\text { Corresponds to } \beta \text { in the EDDM algorithm, used } \\
\text { in DDD's driftDetect function (default }=0.9 \text { ) }\end{array}$ \\
\hline warning_level & $\begin{array}{l}\text { Corresponds to } \alpha \text { in the EDDM algorithm, used } \\
\text { in DDD's driftDetect function (default }=0.95 \text { ) }\end{array}$ \\
\hline decision_rule & $\begin{array}{l}\text { Four voting methods are available, used a method } \\
\text { that incorporates the weight on the old low } \\
\text { diversity ensemble }\end{array}$ \\
\hline weight_oldlow & $\begin{array}{l}\text { Weight for the old low diversity ensemble. Used } \\
\text { for false positive testing (default }=1 \text {, higher if } \\
\text { more false positive concept drifts are expected). }\end{array}$ \\
\hline *reset_after_drift & $\begin{array}{l}\text { Upon drift detection reset accuracies and do not } \\
\text { create two extra ensembles }(1=\text { Yes, } 0=\text { No })\end{array}$ \\
\hline$* M T S$ & $\begin{array}{l}\text { Maximum time step parameter. Limits the time } \\
\text { steps four ensembles are used. }\end{array}$ \\
\hline
\end{tabular}

Note. *New parameters created for this study

A second program, called MergeFiles.py, was created to consolidate the 30 data files generated from the 30 command lines created in CreateBatchFile.py. Each of these 30 data files represented a run of the artificial dataset being tested. MergeFiles.py took all 30 of these result files and merged them into a TXT file for later analysis.

\section{Detailed Test Plan}

Following are the detailed steps I took for this study.

1. Added the MTS parameter to the DDD algorithm.

2. Created an option in the DDD algorithm that reset the learning system when concept drift was encountered. 
3. Used CreateBatchFile.py to build the batch files to test the MTS settings on the artificial datasets. Thirty tests were done on each parameter using the parameter values $31,50,75$, and 100. Each parameter value was tested on the nine severity/speed options for each dataset. Since it was projected that this process would require:

30 Runs X 9 Severity/Speed Settings X 4 MTS parameter values X 6 datasets X 180 seconds giving $\cong 1,166,400$ seconds or 324 hours

A group of computers was used so tests could be done concurrently. Since accuracy was used for comparison, as opposed to time, speed variations in computers was not an issue. Several comparisons were made using the same input data on different computers to be certain the same accuracy was obtained, regardless of computer.

4. Used MergeFiles.py to create a TXT file containing the results of the 30 runs for each severity/speed setting for each MTS parameter setting for each artificial dataset.

5. Used CreateBatchFile.py to build the batch files to test the three real world datasets. As with the artificial data, 30 tests were done on each MTS parameter using the values $31,50,75$, and 100 . Since speed was not examined in this study, only a subset of the original datasets for Electricity, KDD Cup 1999, and Forest CoverType was used for training and testing. For each of the three real world datasets, the training file consisted of 2000 records, taken from the first $80 \%$ of the original real world dataset. The test file consisted of 500 records, taken from the last $20 \%$ of the original real world dataset.

6. Used MergeFiles.py to create a TXT file of the results of the 30 runs for each MTS parameter setting for each of the real world datasets.

7. Applied steps 3 through 6 to the original DDD algorithm and to a version of the DDD algorithm where the learning system reset upon concept drift detection.

8. Determined the MTS parameter value giving the best accuracy for each artificial dataset and each severity/speed setting. Compared the result using this best MTS parameter with the original DDD algorithm and DDD algorithm that resets when concept drift is detected. Note that the MTS value that gave the best accuracy could be different for different severity/speed settings.

9. Tested the impact of changes in $W$ parameter (weight on the old low diversity ensemble) relative to settings in the MTS on the artificial data. The best setting of the MTS parameter was compared using $W=1, W=2$, and $W=3$ to determine the impact of $W$ on accuracy when false positive concept drifts were found. A false positive concept drift was forced halfway through the first concept and halfway through the second concept for this test. These results were compared against the original DDD algorithm with the same $W$ settings. 
10. Tested the impact of changes in $W$ parameter (weight on the old low diversity ensemble) relative to settings in the MTS on the real world data. The best setting of the MTS parameter was compared using $W=1, W=2$, and $W=3$ to see the impact of $W$ on accuracy when false positive concept drifts are found. False positive concept drift was forced periodically for this test. These results were compared against the original DDD algorithm with the same $W$ settings.

\section{Resources Used}

The DDD code (written in C/C++) used by Minku and Yao (2012) was available and was used as the basis for this study. Bloodshed Dev C++ was used as the IDE for $\mathrm{C} / \mathrm{C}++$ programming. Since accuracy and not speed was the focus of this study, a group of computers was used to run the various datasets under the different settings. Using multiple computers greatly sped up the testing process. Here is a list of the resources utilized for this study:

- Computer Lab Computers

- Personal Computer

- $\mathrm{C} / \mathrm{C}++$ Compiler and IDE

- Microsoft Excel (used to compare results with Python calculations)

- Python and Matplotlib

\section{Summary}

An overview of the methodology for this study has been presented. The impact of an MTS parameter on the DDD algorithm was studied in detail using artificial and real world data. The impact of false positive concept drifts was explored by comparing the Best MTS and the original DDD with different values for the $W$ parameter (weight on the old low diversity ensemble) on artificial and real world datasets. 


\section{Chapter 4}

\section{Results}

\section{Introduction}

This chapter shows the results of the methodology detailed in Chapter 3. The chapter is organized by each test completed, with the results from both artificial and real world datasets given. The artificial test results will begin with four Circle datasets. These four Circle datasets will be a running example for all tests shown. After this, graphs showing interesting results will be given. The results on artificial datasets will then be summarized. The real world results will follow. The first test conducted was designed to determine the best MTS for each dataset. The best MTS found for each dataset was then used in a direct comparison to the original DDD algorithm and to the original DDD algorithm that resets the learning system when concept drift is found. The results of these comparisons are represented graphically. Also, $t$-tests were used to provide an additional measurement between the original DDD algorithm and the DDD algorithm using the best MTS. Finally, a comparison showing the average number of time steps four ensembles were used to predict accuracy was made between the best MTS version of the DDD and the original DDD algorithm. This comparison was used to help prove that the best MTS version of the DDD is more time efficient than the original DDD, making the MTS version superior when the prequential accuracies of the two algorithms were similar.

For the secondary portion of my research, results are shown that identify the impact of changing $W$, the weight on the old low diversity ensemble. The purpose of $W$ is to help keep the algorithm from reacting to what appears to be concept drift but is not (false positive concept drift). These results will be shown for artificial and real world 
datasets. For the artificial data, the results using the same four Circle datasets will be shown. Following this will be results illustrating interesting results of the changes in $W$. Results for the real world data will also be given. These tests were completed using the best $M T S$ value found for each dataset and were compared to the original DDD algorithm. The various $W$ settings are compared graphically.

\section{Determine Best MTS}

For each of the nine settings of each artificial dataset (e.g. Circle Low Severity, High Speed) the MTS values 31, 50, 75, and 100 were used for comparison to determine the MTS that produced the highest prequential accuracy. The selection of 31 was used because 31 is the minimum possible for the DDD, since the DDD algorithm requires the four ensembles to be in memory at least 30 time steps. The other three MTS values (50, $75,100)$, where chosen because they are the next three multiples of 25 after the minimum value of 31. This comparison was done graphically. If greater than one MTS value produced the highest prequential accuracy, the $M T S$ with the lowest value was chosen as the best MTS.

Following are the MTS comparisons with four of the Circle datasets. For all of the artificial datasets, accuracy was reset half-way through the data (for Circle time step 1000) as was done by Minku and Yao (2012). In addition, a single concept drift occurred with each dataset, typically early in the second half of the dataset. 
Figure 4. Circle - Low Severity/High Speed, MTS Comparison

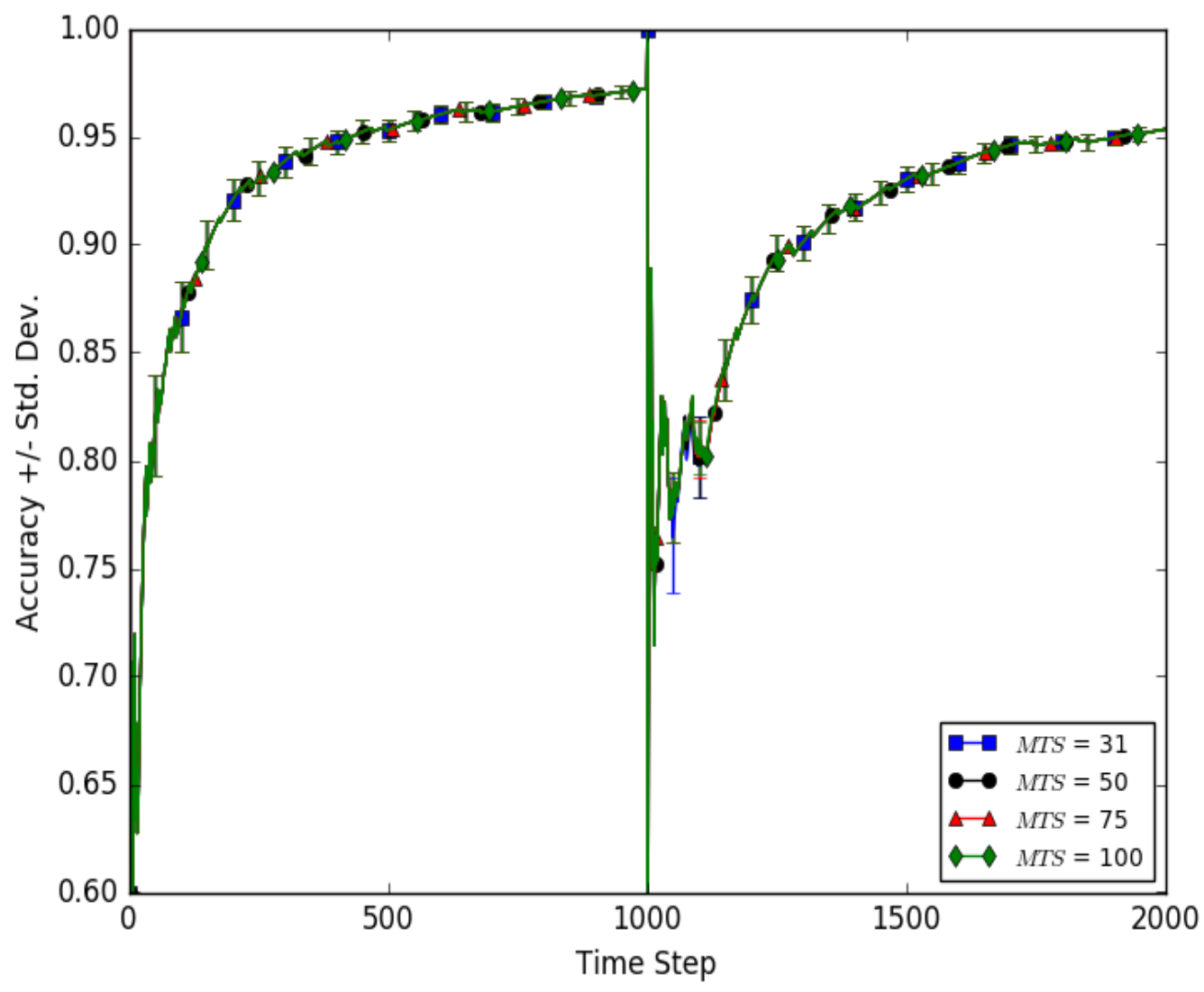

Figure 4. Comparison of MTS settings using the Circle dataset. Low severity and high speed concept drift were used.

Figure 4 shows the results from Circle dataset where severity is low and speed is high. As can be seen, all four MTS values in Circle Low Severity, High Speed gave essentially the same prequential accuracy and all performed well. As a result, 31 was chosen as the lowest MTS value for this dataset since in the case of a tie with MTS, the $M T S$ with the lowest value was chosen, since this choice allowed the four ensembles to be in memory a shorter amount of time.

The results from Circle where both severity and speed are low are found in Figure 5. 
Figure 5. Circle - Low Severity/Low Speed, MTS Comparison

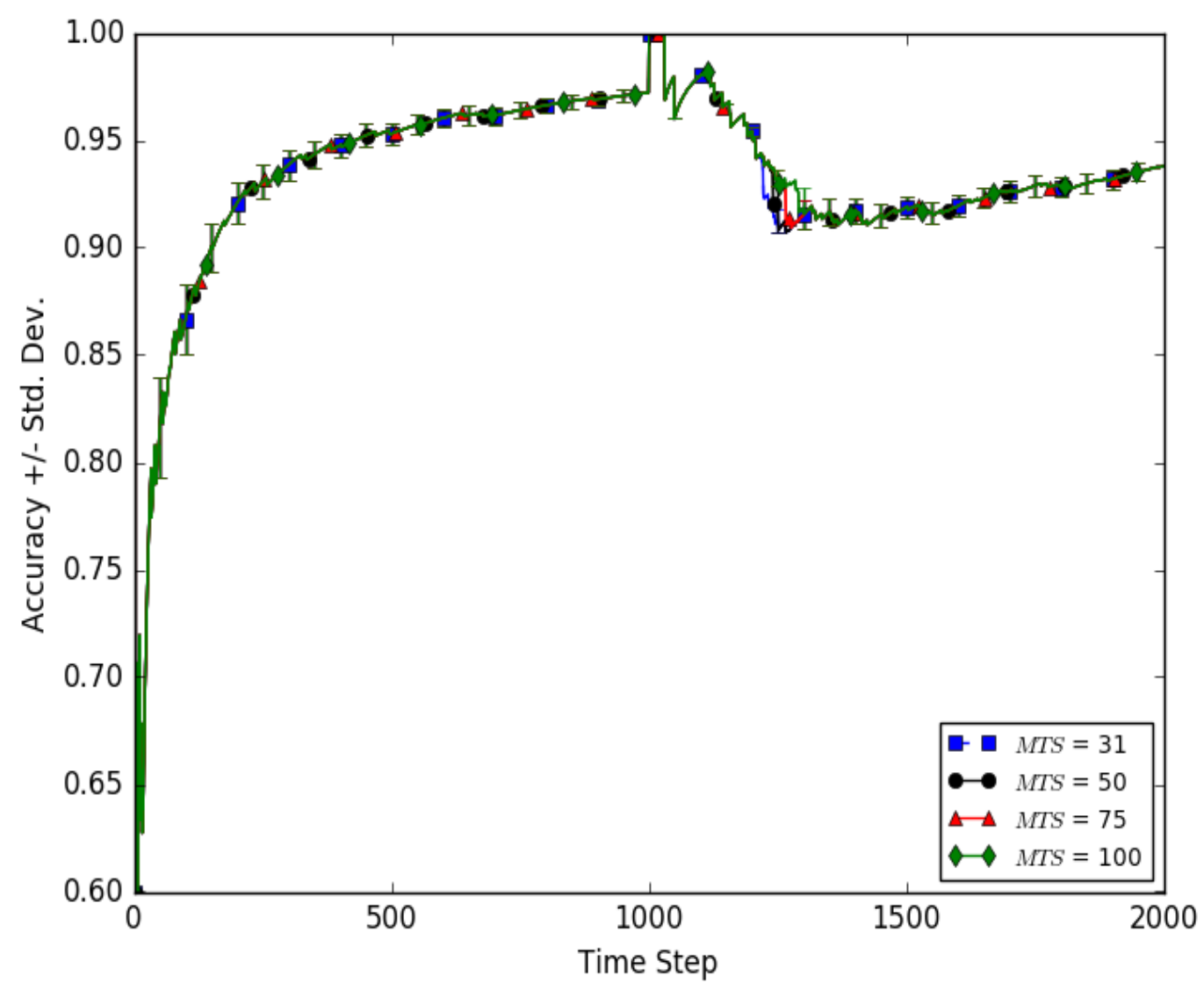

Figure 5. Comparison of MTS settings using the Circle dataset. Low severity and low speed concept drift were used.

The graph in Figure 5 is for Circle Low Severity, Low Speed. The four MTS values are very close, but they separate in time steps 1100-1400. Figure 6 shows a magnified view of this area. 
Figure 6. Circle - Low Severity/Low Speed, MTS Comparison (Magnified)

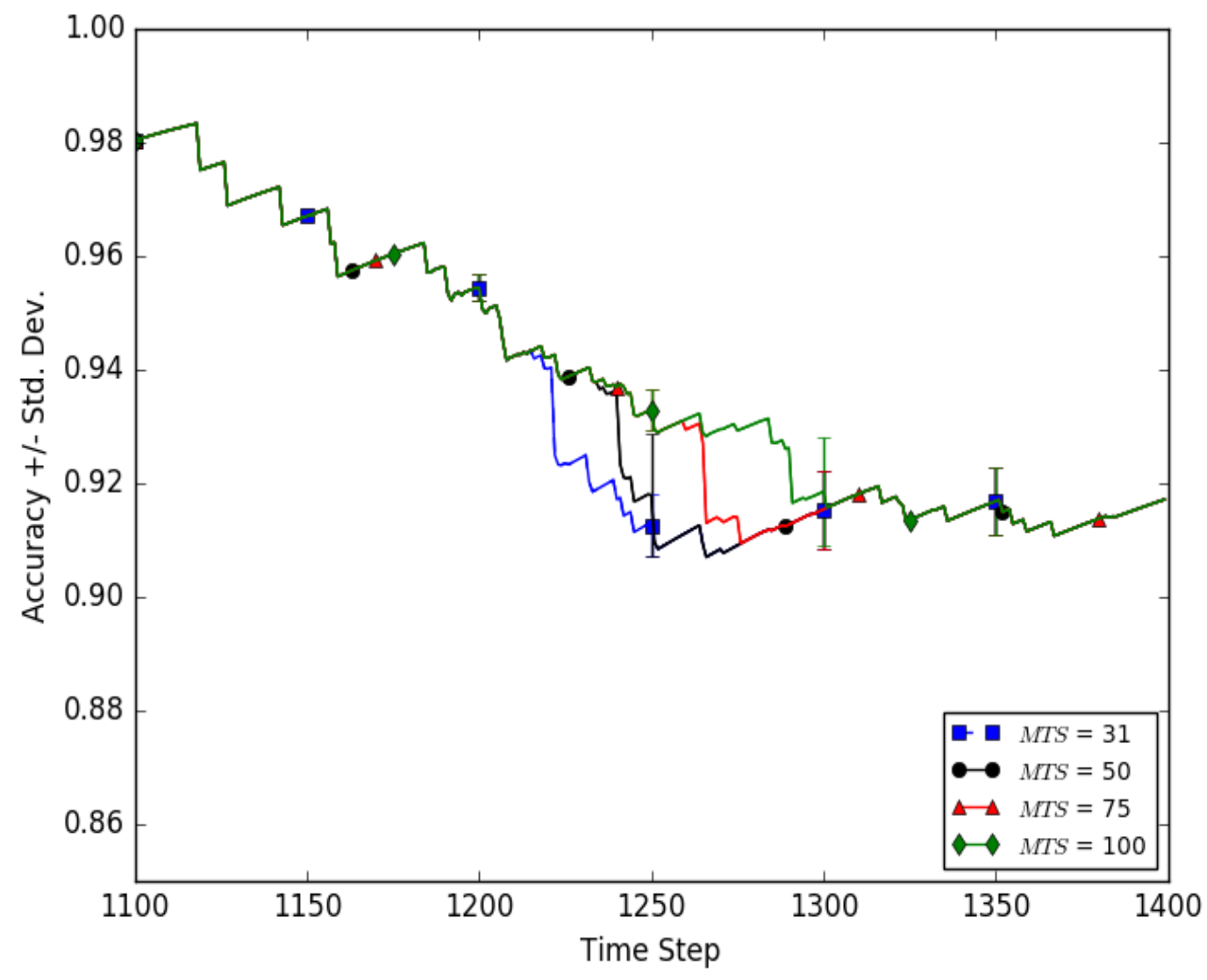

Figure 6. Comparison of MTS settings using the Circle dataset. Low severity and low speed concept drift were used. This view is magnified during the time the MTS results separate.

It can be seen in Figure 6 that MTS 100 produced a higher average prequential accuracy around time steps 1260-1300. For this dataset MTS 100 was selected as the best MTS. The next comparison (in Figure 7), looked at the impact of changing the severity and speed from low to high. 
Figure 7. Circle - High Severity/High Speed, MTS Comparison

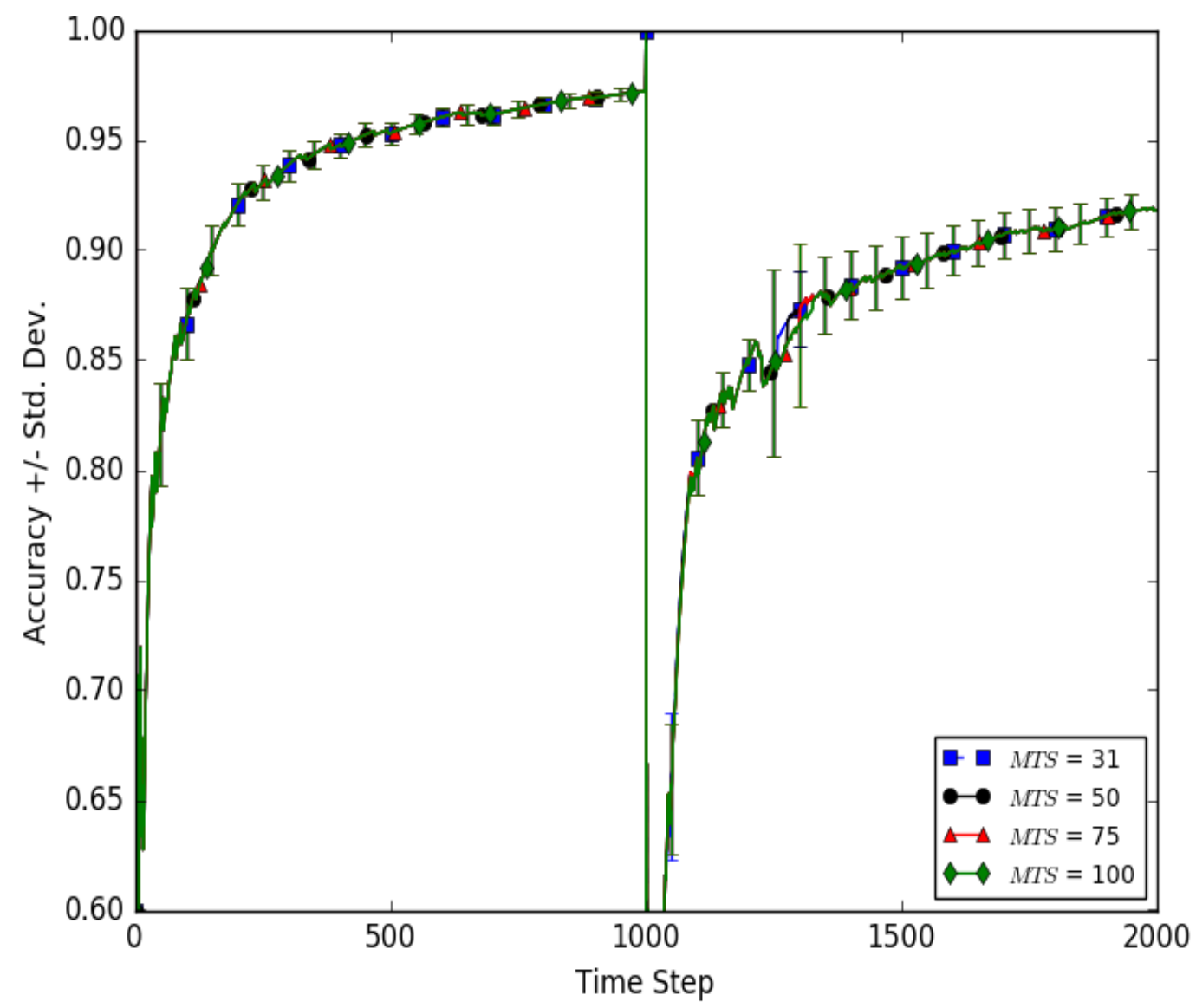

Figure 7. Comparison of MTS settings using the Circle dataset. High severity and high speed concept drift were used.

As Figure 7 shows, the $M T S$ values 31, 50, and 75 were very close throughout. Results from using the DDD with various MTS values on this dataset are magnified in Figure 8. 
Figure 8. Circle - High Severity/High Speed, MTS Comparison (Magnified)

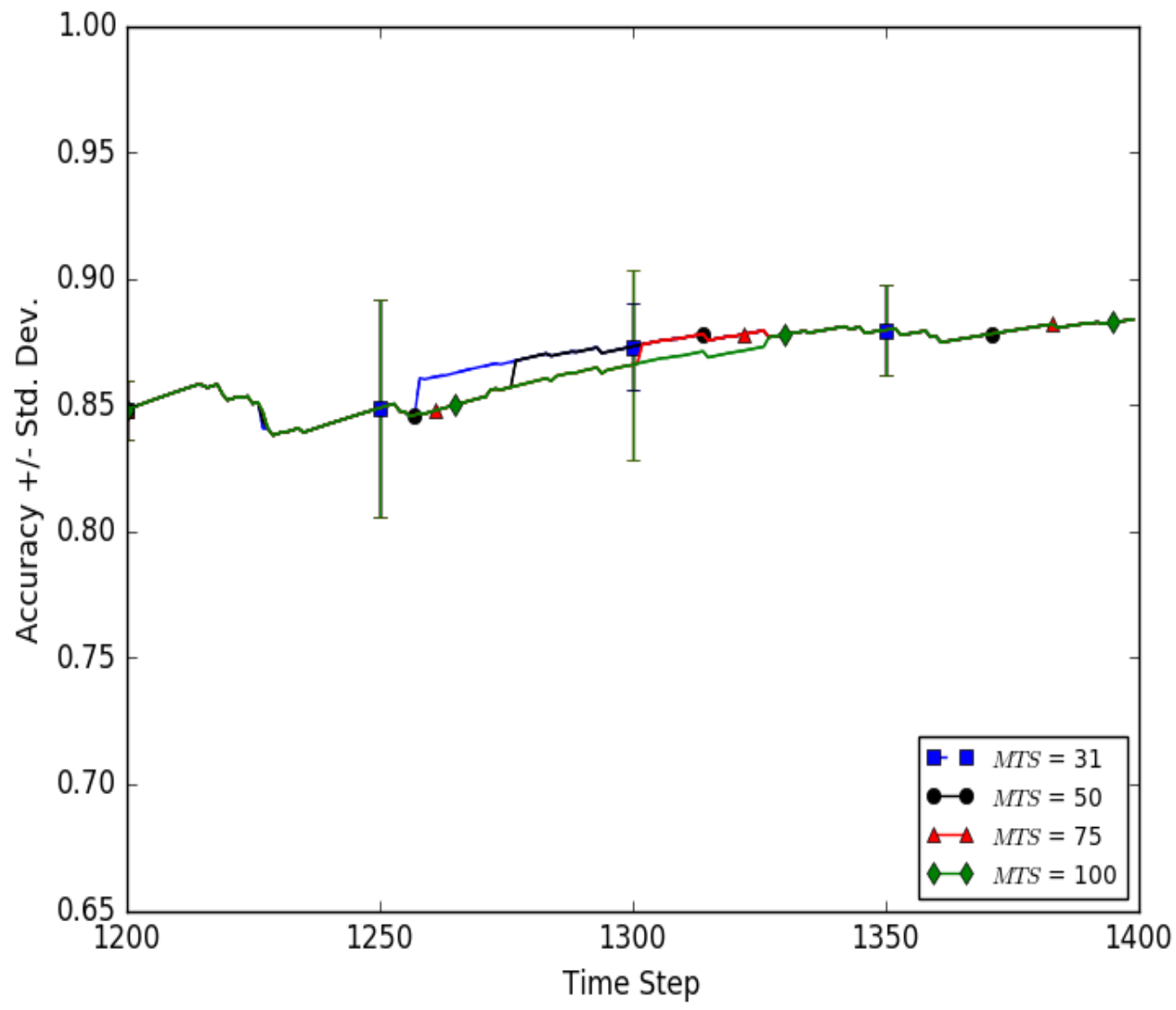

Figure 8. Comparison of MTS settings using the Circle dataset. High severity and high speed concept drift were used here. This view is magnified during the time the MTS results separate.

MTS 31 was chosen as the best MTS. MTS 100 was a little lower around time steps 1260-1335 and MTS 31 had the highest consistent prequential accuracy. The fourth Circle comparison is shown in Figure 9. 
Figure 9. Circle - High Severity/Low Speed, MTS Comparison

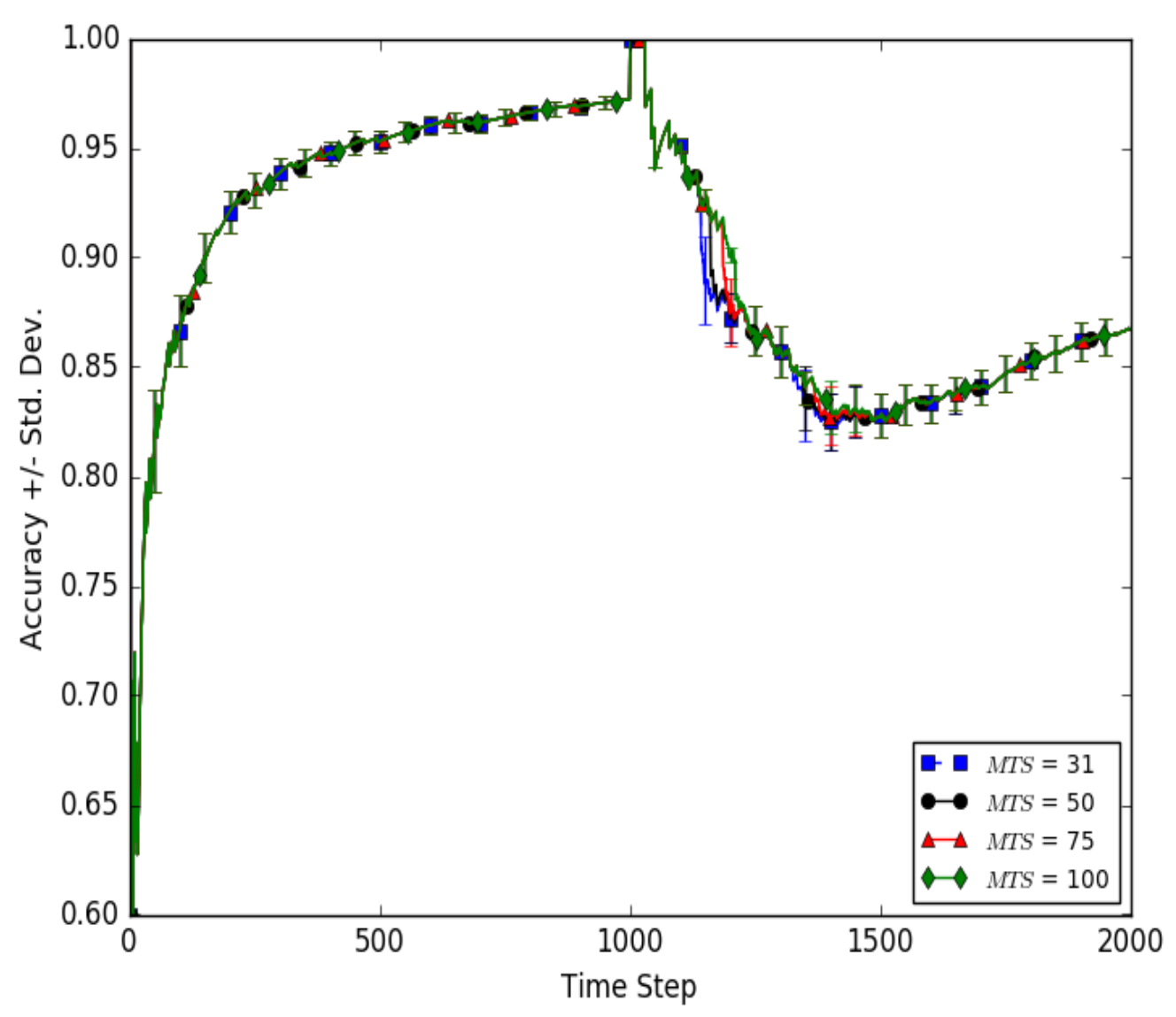

Figure 9. Comparison of MTS settings using the Circle dataset. High severity and low speed concept drift were used.

In Figure 9, the Circle dataset was tested with severity remaining high and speed changing to low. It can be seen that MTS 100 had the highest consistent accuracy throughout. The accuracy of the other MTS values fell around time steps 1100-1200.

Following are several other artificial test cases that illustrate interesting results regarding the $M T S$ values. 
Figure 10. SineV - High Severity/Medium Speed, MTS Comparison

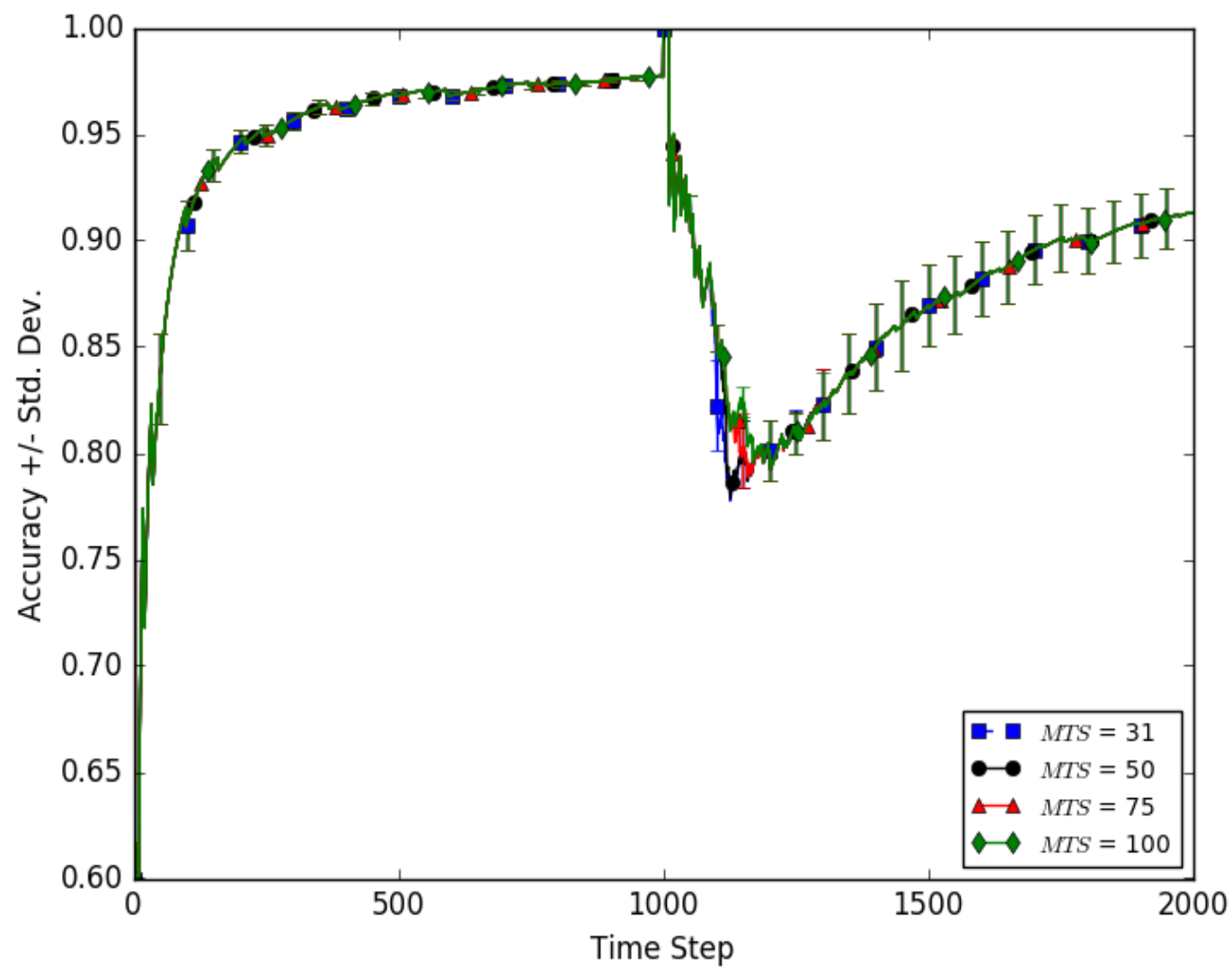

Figure 10. Comparison of MTS settings using the SinV dataset. High severity and Medium speed concept drift were used.

As Figure 10 shows, SineV high severity and medium speed had better accuracy with MTS 100 after the concept drift. However, the prequential accuracies from the other options were very close. MTS 100 had the best overall accuracy for this dataset. Figure 11 gives the results of SineH where severity is high and speed is low. 
Figure 11. SineH - High Severity/Low Speed, MTS Comparison

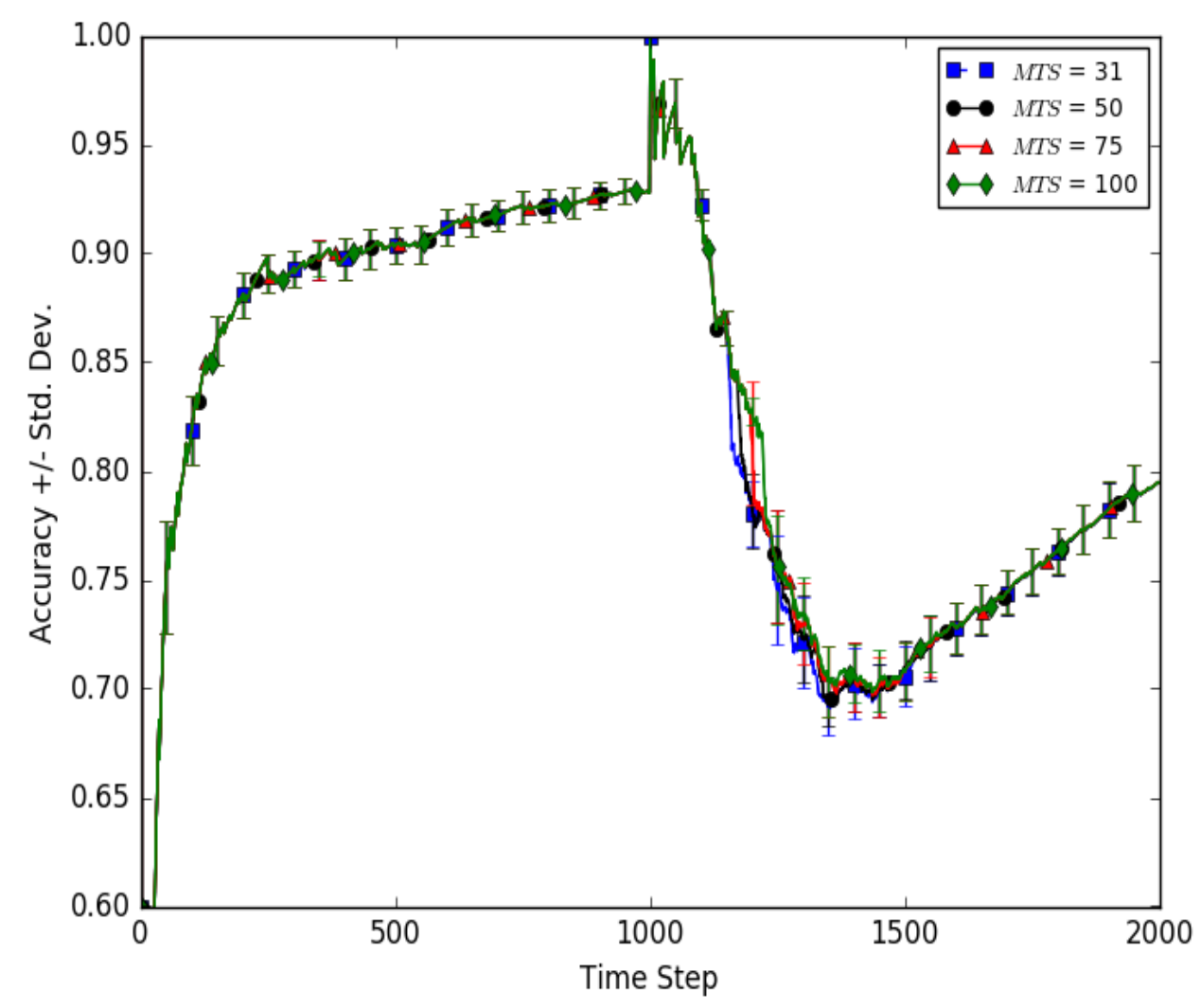

Figure 11. Comparison of MTS settings using the SineH dataset. High severity and low speed concept drift were used.

Using SineH with high severity and low Speed, Figure 11 reveals that the four MTS values gave slightly different results after the concept drift around time steps 11501400, with MTS 100 showing the best accuracy. Figure 12 provides the results where severity is high and speed is medium with the Plane dataset. 
Figure 12. Plane - High Severity/Medium Speed, MTS Comparison

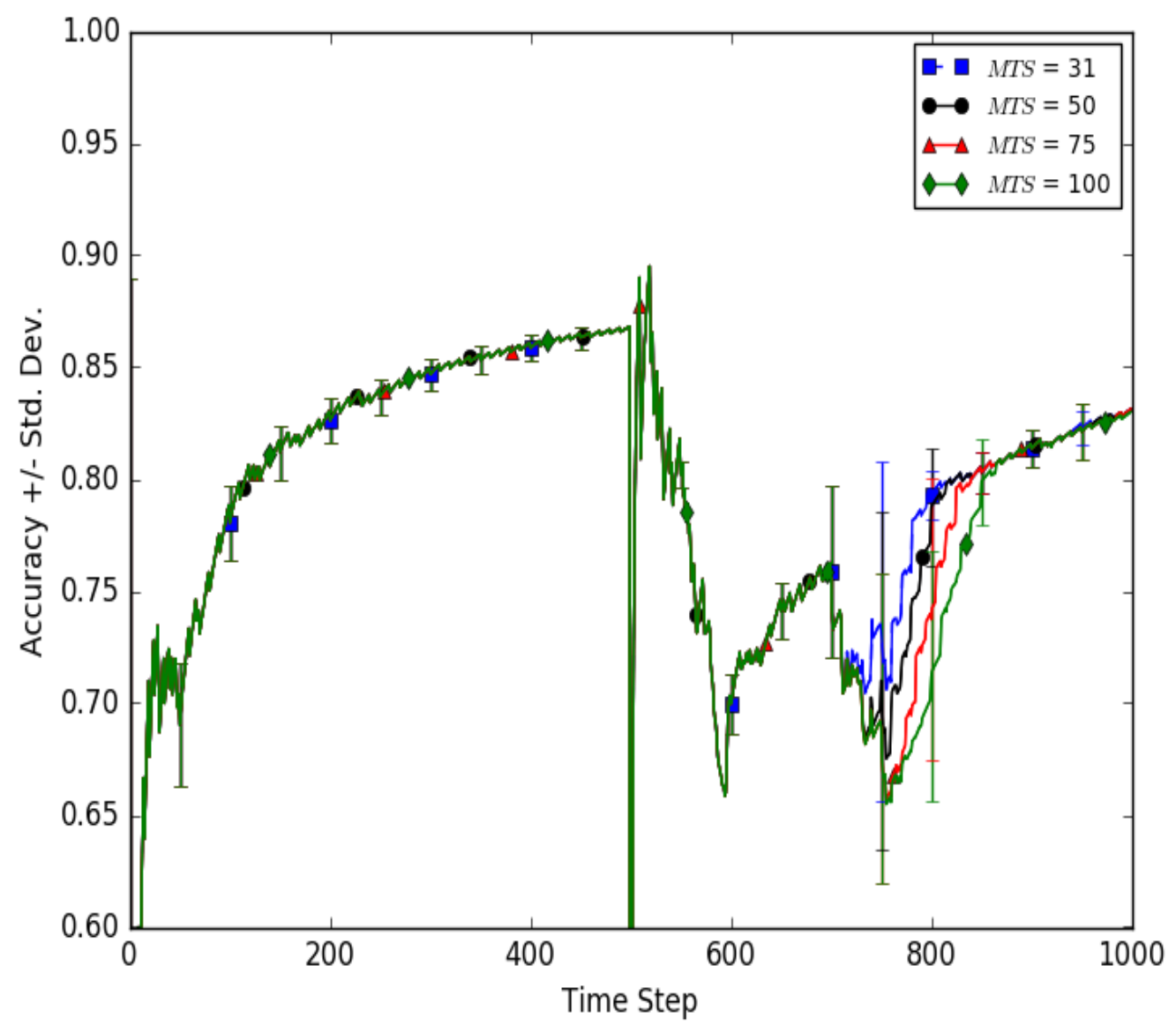

Figure 12. Comparison of MTS settings using the Plane dataset. High severity and medium speed concept drift were used.

Figure 12 shows an interesting result with the Plane dataset with high Severity and medium speed. At approximately time steps 725-875, all four MTS values increased in accuracy, but not at the same time. For this dataset MTS 31 had the best overall accuracy. 
Figure 13. Boolean - High Severity/High Speed, MTS Comparison

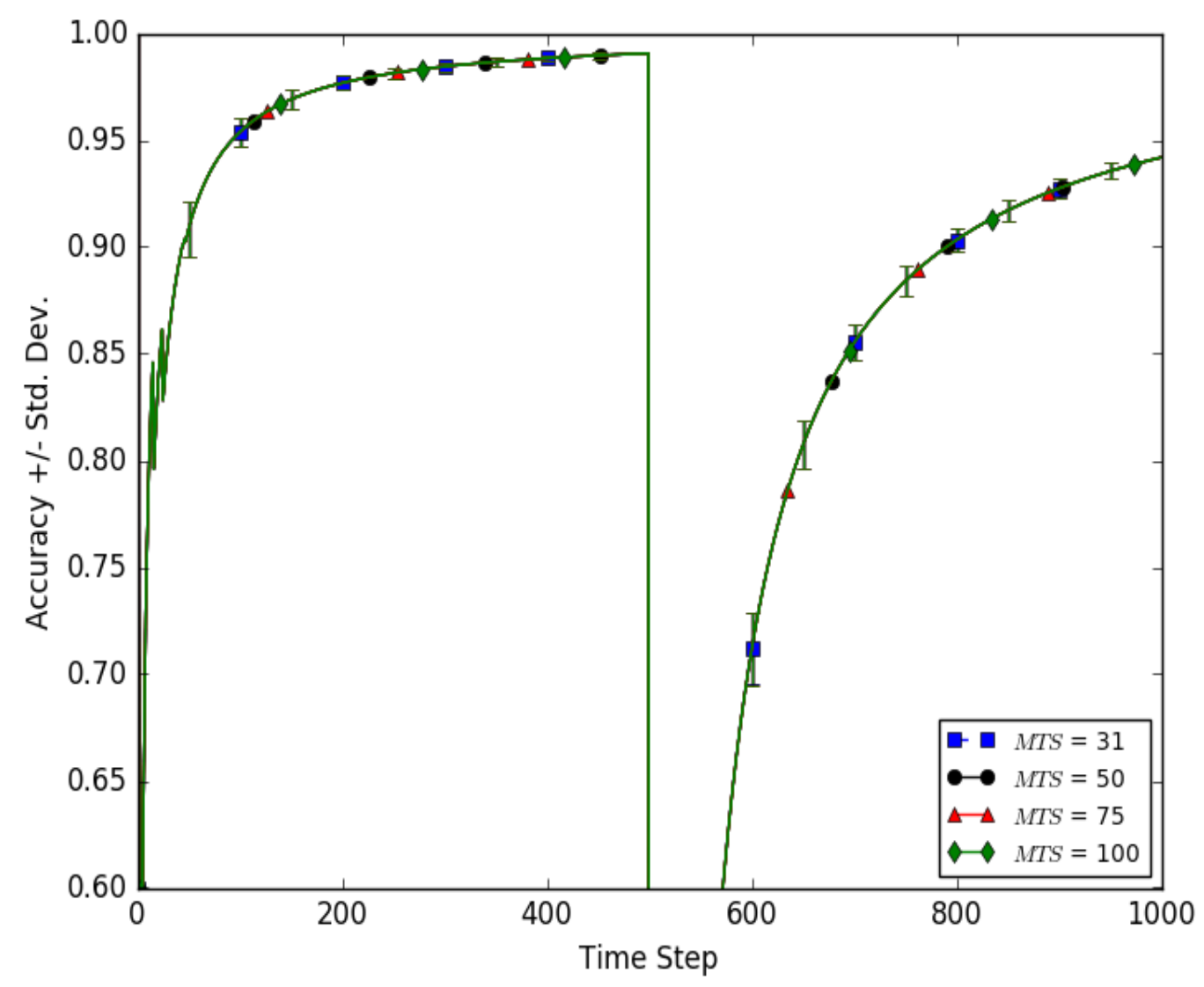

Figure 13. Comparison of MTS settings using the Boolean dataset. High severity and high speed concept drift were used.

Figure 13 illustrates that while some MTS values give different accuracies, some, like Boolean, high severity, high speed, gave essentially the same results for the MTS values $31,50,75$, and 100 . In cases where the prequential accuracies computed from different MTS values were identical, MTS 31 was chosen as the best MTS because it produced the same prequential accuracy in fewer time steps.

The graphs for all of the other 54 artificial datasets are included in the appendix. Figure 14 provides a frequency distribution of MTS values selected with all 54 artificial datasets. 
Figure 14. Frequency of MTS Values for Artificial Data

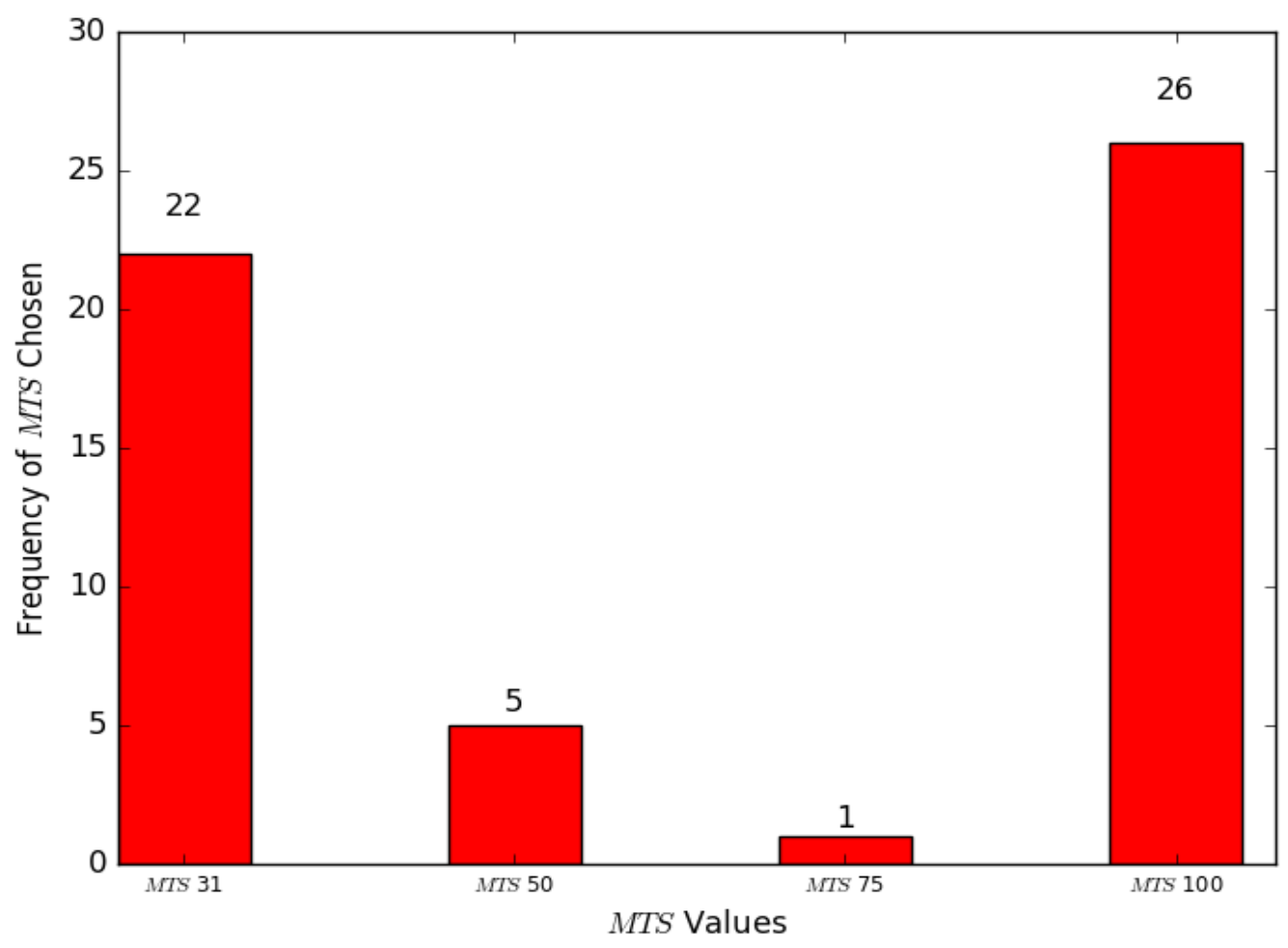

Figure 14. Breakdown of the MTS values chosen for the artificial data

As can be seen in Figure 14, the two most common MTS values selected were 31 and 100. Generally, MTS 100 was chosen because it outperformed the other MTS values, although the four options were close in accuracy in most cases. MTS 31 was selected frequently because there were cases when all four MTS options produced similar results, so the lowest MTS value was selected. In the case of the Line dataset with medium severity and low speed, it was interesting to note that the accuracy of MTS 75 was just slightly less than MTS 100. MTS 100 was selected as the best MTS but it did raise the question of how to resolve the trade-off between accuracy and the additional time required where four ensembles were used. It was also interesting to observe that cases existed where a lower amount of assistance (lower MTS) seemed to provide better 
accuracy. Following are the results from the MTS comparison using real world data. As with the artificial data, accuracies were reset halfway through the data.

\section{Figure 15. Electricity, MTS Comparison}

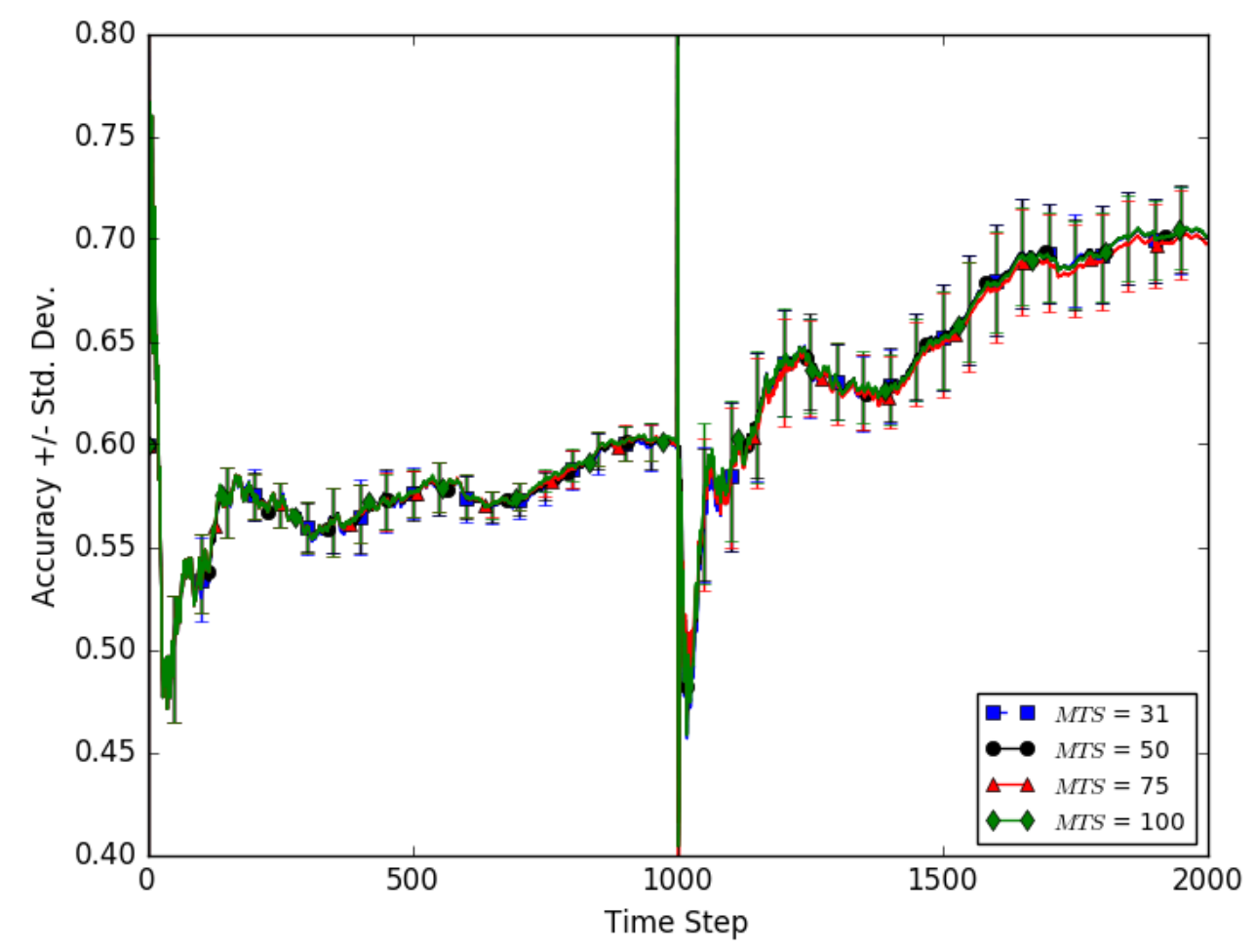

Figure 15. Comparison of MTS settings using the Electricity dataset.

As seen in Figure 15, with the Electricity dataset there were only slight variations in the four MTS values. MTS 75 was slightly higher after the accuracies were reset, but MTS 100 was higher around time step 1100 and also around time step 1900. MTS 100 was selected as the best MTS in this case. 
Figure 16. Forest CoverType, MTS Comparison

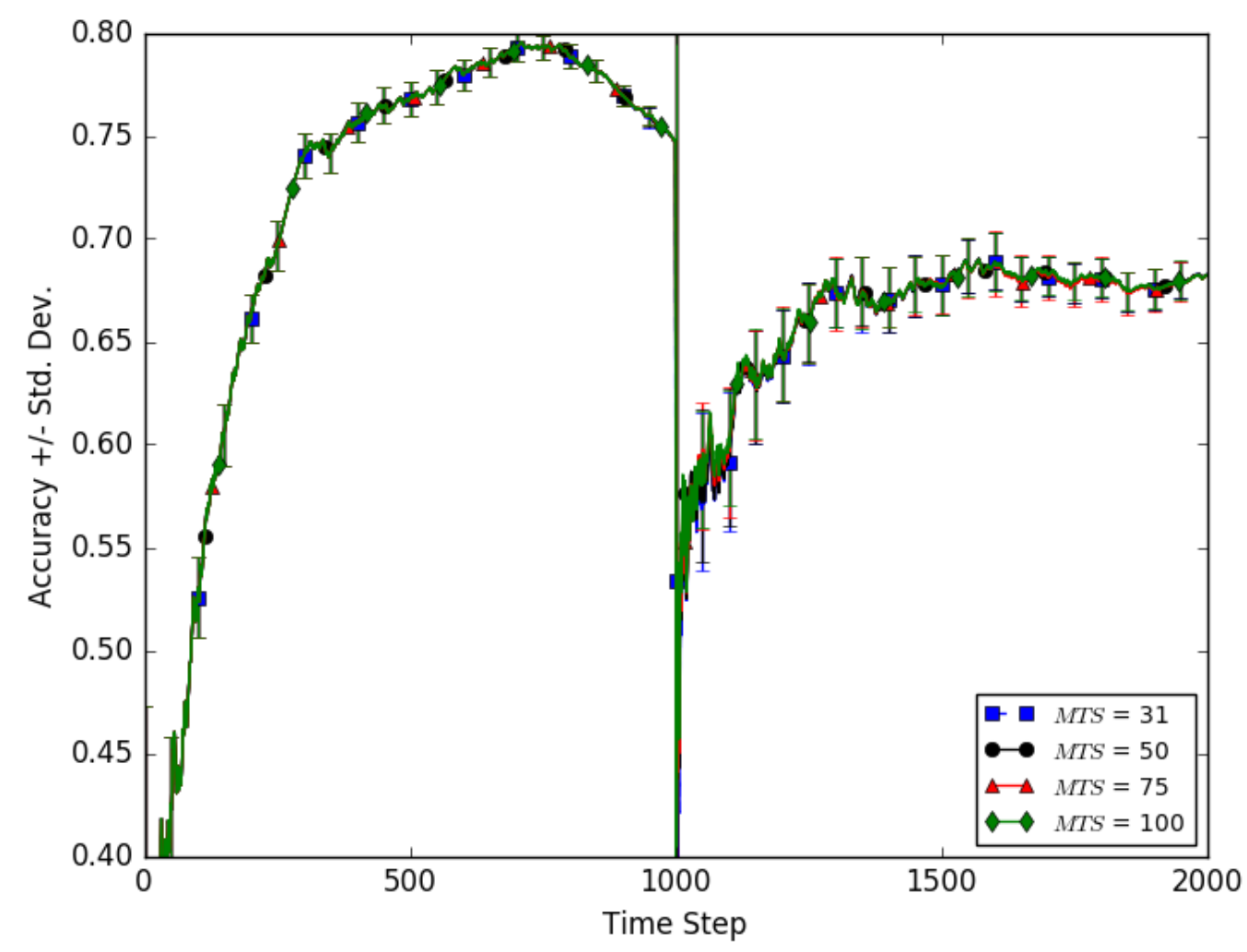

Figure 16. Comparison of MTS settings using the Forest CoverType dataset.

As with the Electricity dataset, Figure 16 shows that Forest CoverType had similar accuracies for each MTS value tested. After accuracies were reset halfway through Forest CoverType, MTS 100 provided slightly better results. Therefore, MTS 100 was chosen as the best MTS for Forest CoverType. The KDD 1999 dataset follows in Figure 17. 
Figure 17. KDD 1999, MTS Comparison

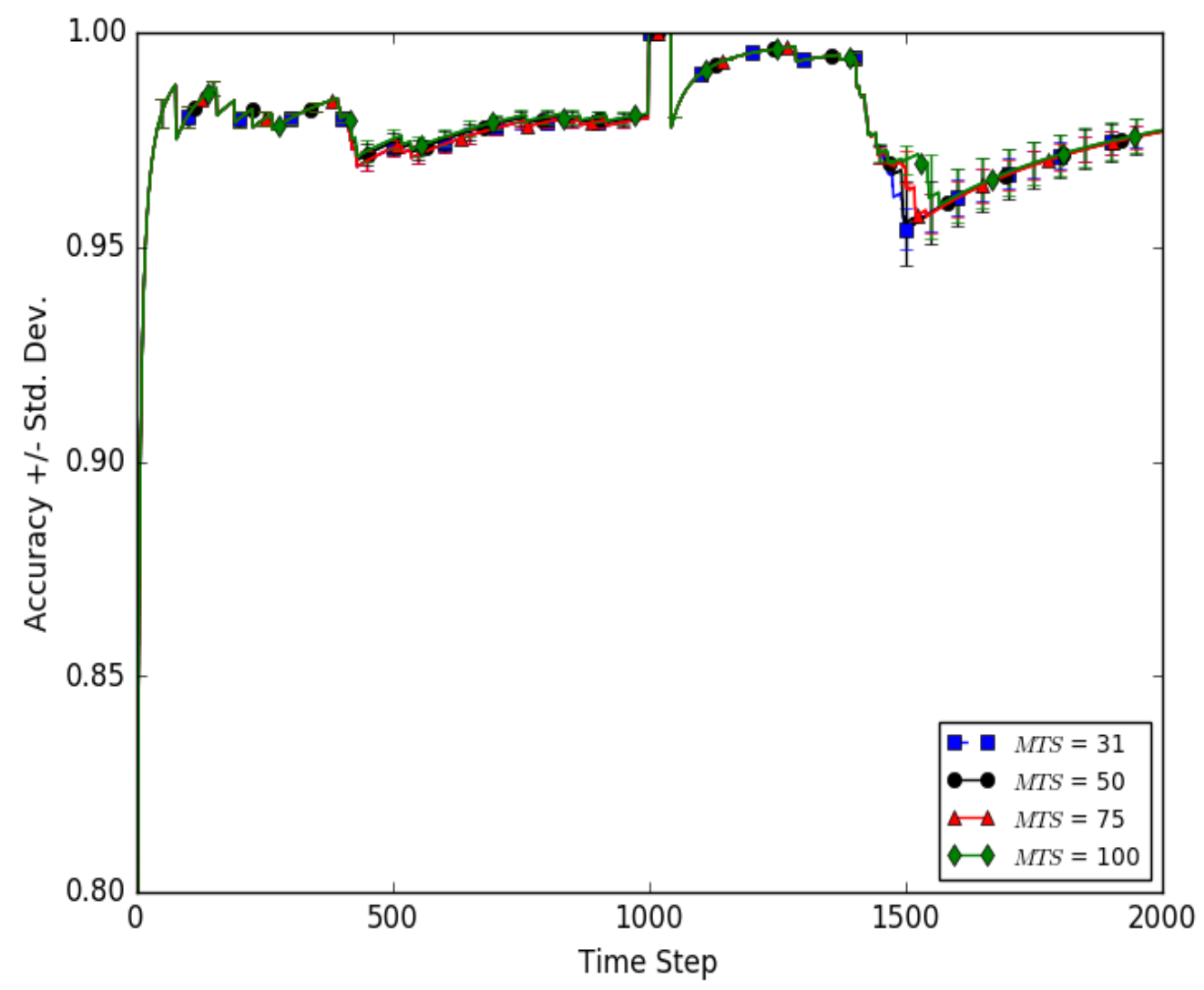

Figure 17. Comparison of MTS settings using the KDD 1999 dataset.

As Figure 17 shows, the KDD 1999 dataset had four MTS values that are very similar until around time step 1500, when MTS 100 provided the highest accuracy. MTS 100 was chosen as the best MTS for KDD 1999.

For the three real world datasets, the accuracies produced by the four MTS values were quite close to each other. In all three cases, however, MTS 100 had the best overall accuracy and was chosen as the best MTS for later comparisons. 


\section{Results of Comparison with other Concept Drift Algorithms}

The version of the DDD algorithm using the best MTS was compared to the original DDD algorithm and to a version of the DDD algorithm where the learning system was reset when a concept drift was detected. For convenience, the version of the DDD using the MTS giving the highest prequential accuracy was called Best MTS, the original DDD algorithm was called Standard DDD, and the version of the DDD algorithm which has its learning system reset when a concept drift is detected was called DDD with Reset. For DDD with Reset, accuracies were reset when concept drift occurred. Also, only two ensembles were used with DDD with Reset, as opposed to the four ensembles found in Best MTS and Standard DDD. Accuracy was reset half-way through the data for all three algorithms as was done by Minku and Yao (2012). Using the same format as was done with finding the best MTS, results from four Circle datasets will be shown, followed by selected results from the artificial data. Then summary information will be used to draw conclusions from the results obtained. The outcomes from the real world data will then follow. The first comparison of Best MTS, Standard DDD, and DDD with Reset occurs in Figure 18. 
Figure 18. Circle - Low Severity/High Speed, Comparison of Algorithms

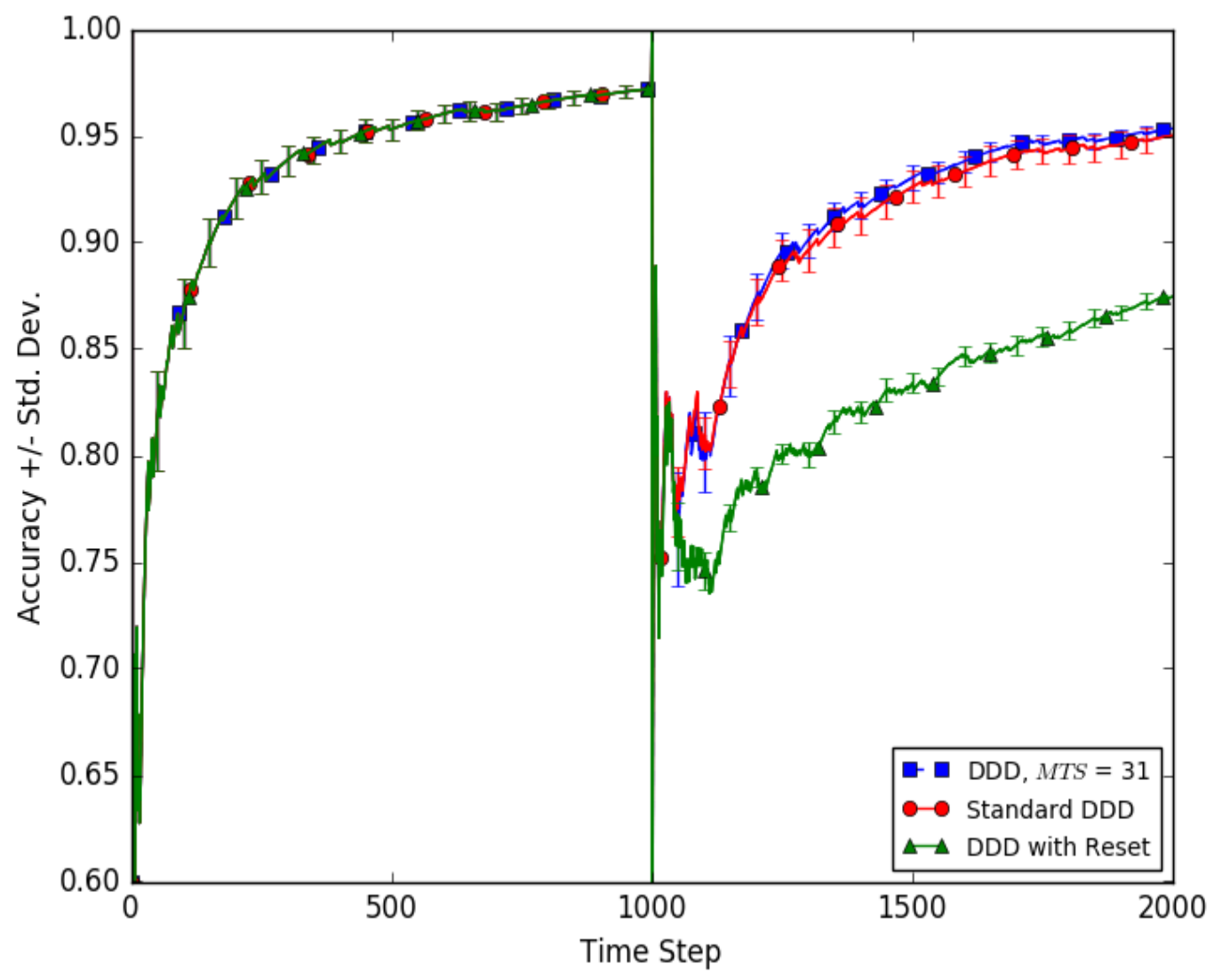

Figure 18. Circle - Low Severity/High Speed, comparison using MTS 31, Standard DDD, and DDD with Reset.

As can be seen in Figure 18, all three algorithms had similar accuracy before the drift, but Best MTS and Standard DDD outperformed DDD with Reset after the drift. In fact, it was noted that the Best MTS algorithm slightly outperformed the Standard DDD as well. 
In Figure 19, all three algorithms had similar accuracy until around time step 1350, when Best MTS and Standard DDD provided the best results. From time step 1600 to 2000 Best MTS gives the highest accuracy.

Figure 19. Circle - Low Severity/Low Speed, Comparison of Algorithms

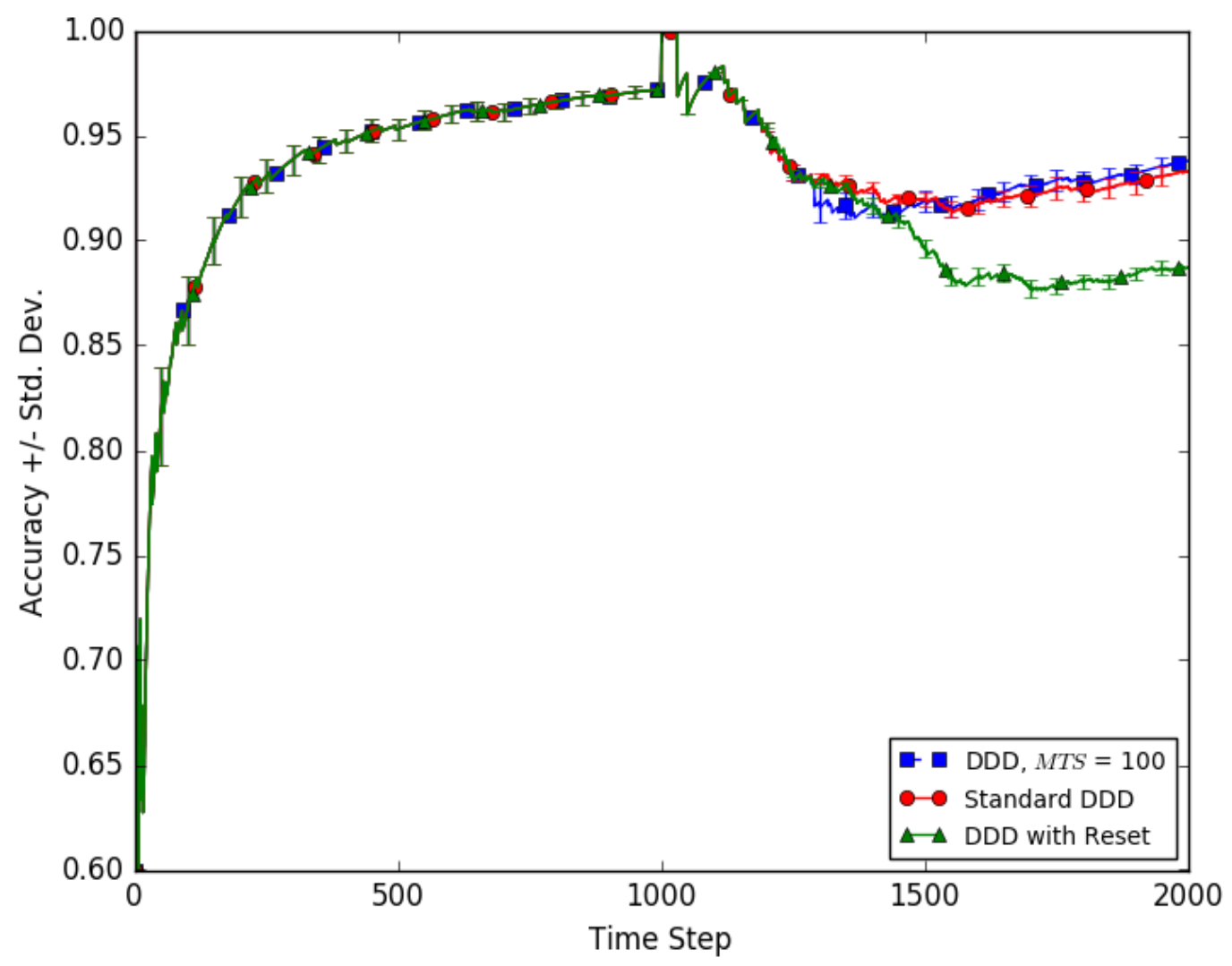

Figure 19. Circle - Low Severity/Low Speed, comparison using MTS 100, Standard DDD, and DDD with Reset. 
Figure 20. Circle - High Severity/High Speed, Comparison of Algorithms

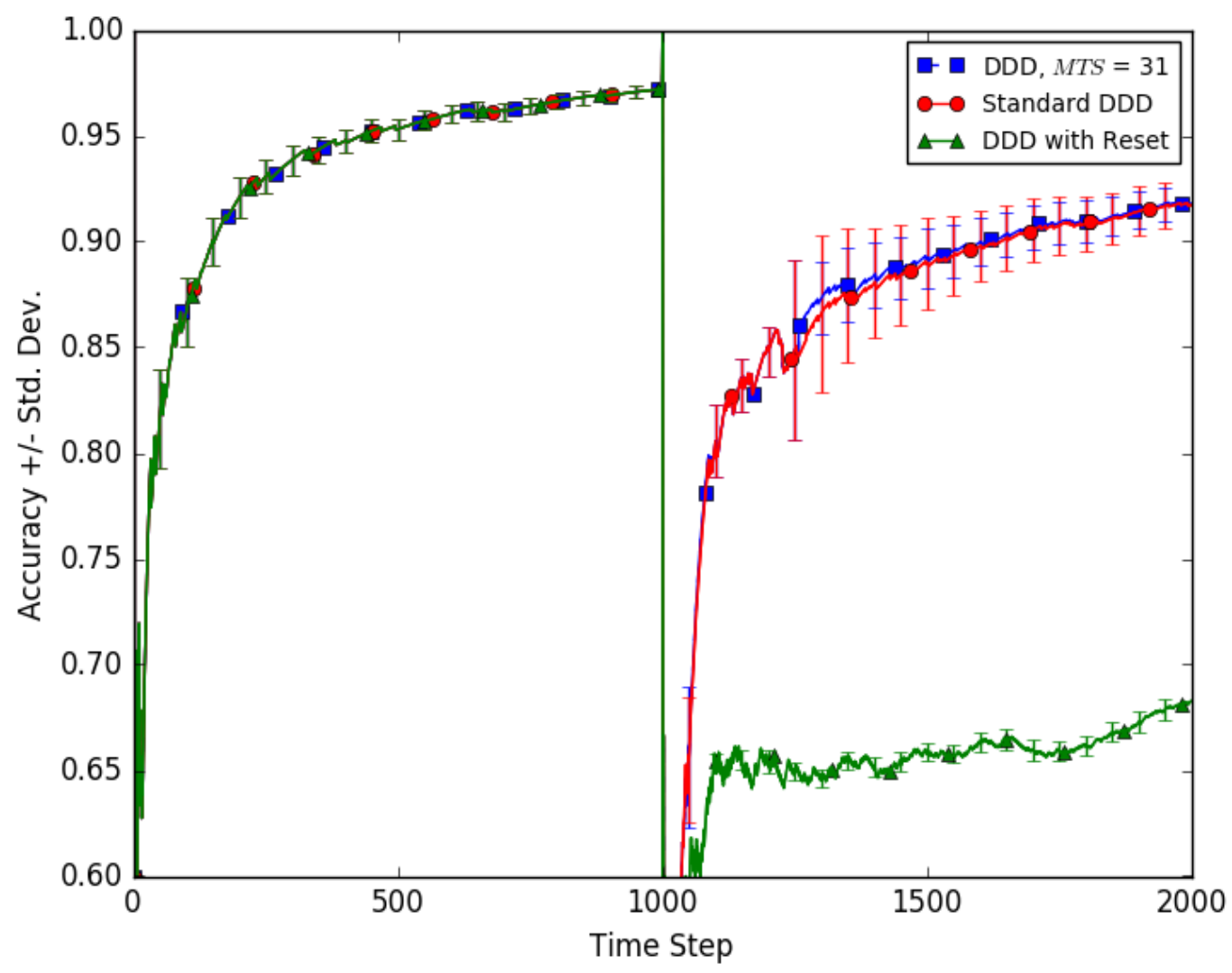

Figure 20. Circle - High Severity/High Speed, Comparison of Algorithms. Circle High Severity/High Speed, comparison using MTS 31, Standard DDD, and DDD with Reset.

In Figure 20, the Best MTS was slightly better than DDD from 1250 to 1400, then these two algorithms were virtually the same. 
Figure 21. Circle - High Severity/Low Speed, Comparison of Algorithms

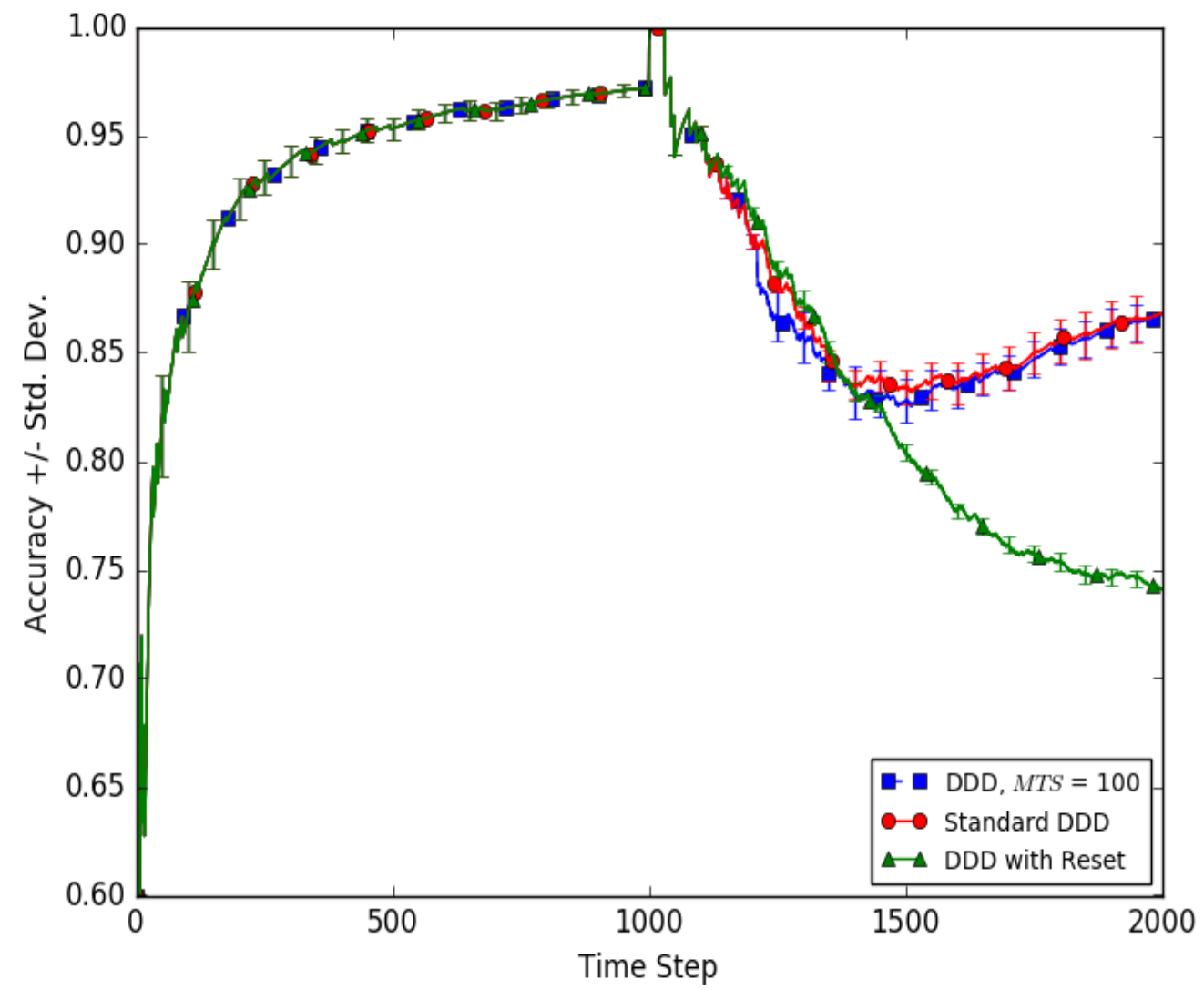

Figure 21. Circle - High Severity/Low Speed, comparison using MTS 100, Standard DDD, and DDD with Reset.

In Figure 21, all three algorithms were similar before the concept drift. After the concept drift, DDD with Reset had the highest accuracy up to time step 1400. Best MTS and Standard DDD then provided the highest accuracy from about time step 1400 to 2000, with Standard DDD slightly higher in accuracy. 
Figure 22. SineV - Medium Severity/High Speed, Comparison of Algorithms

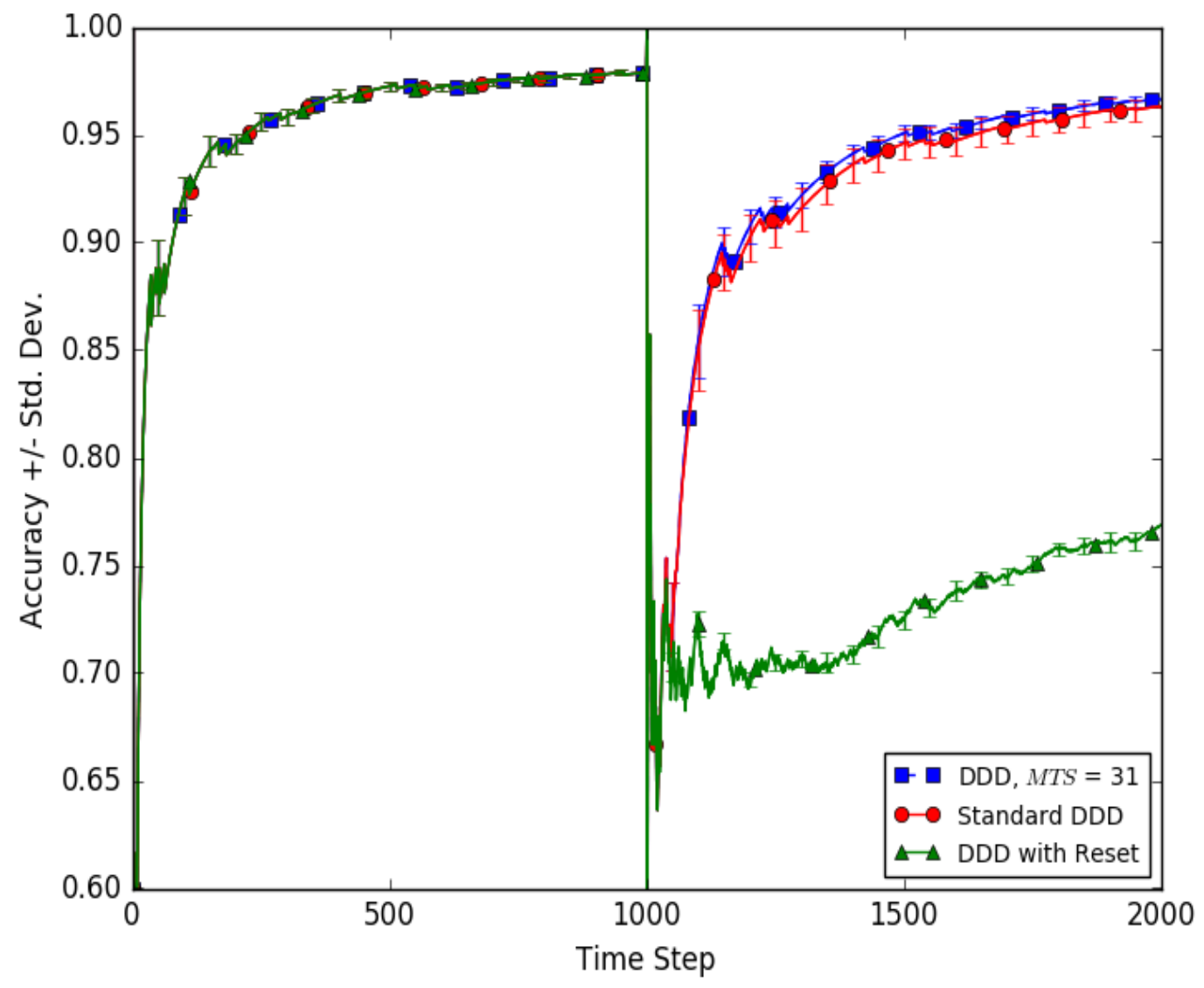

Figure 22. SinV - Medium Severity/High Speed, comparison using MTS 31, Standard DDD, and DDD with Reset.

In Figure 22, Best MTS and Standard DDD provided the highest prequential accuracy, with Best MTS slightly higher than Standard DDD at approximately time steps 1200 to 2000. 
Figure 23. Line - Medium Severity/High Speed, Comparison of Algorithms

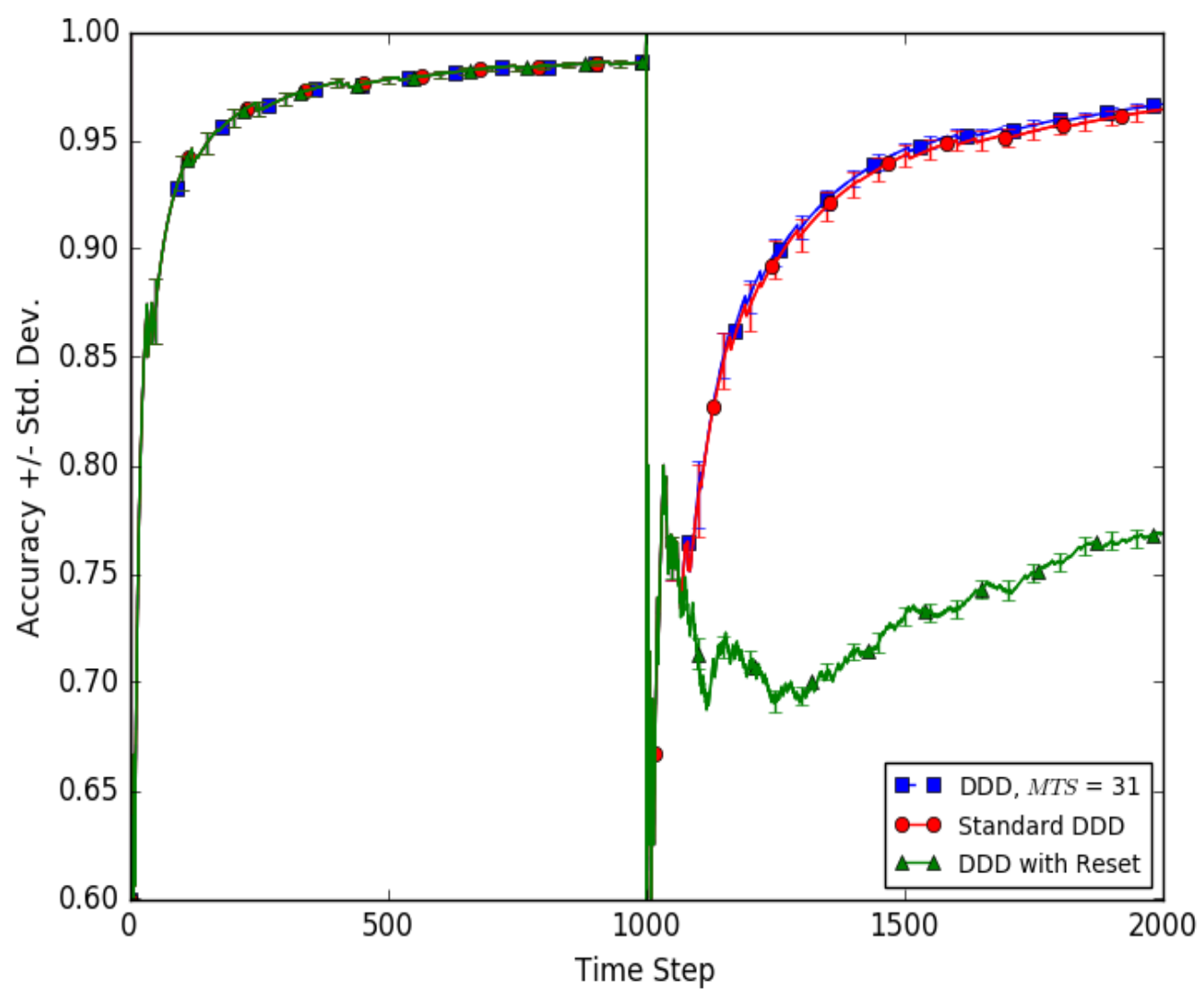

Figure 23. Line - Medium Severity/High Speed, comparison using MTS 31, Standard DDD, and DDD with Reset.

The results in Figure 23 show that Best MTS and Standard DDD gave the best accuracy, with Best MTS only slightly better that Standard DDD at time steps 1350-2000. 
Figure 24. Plane - High Severity/Medium Speed, Comparison of Algorithms

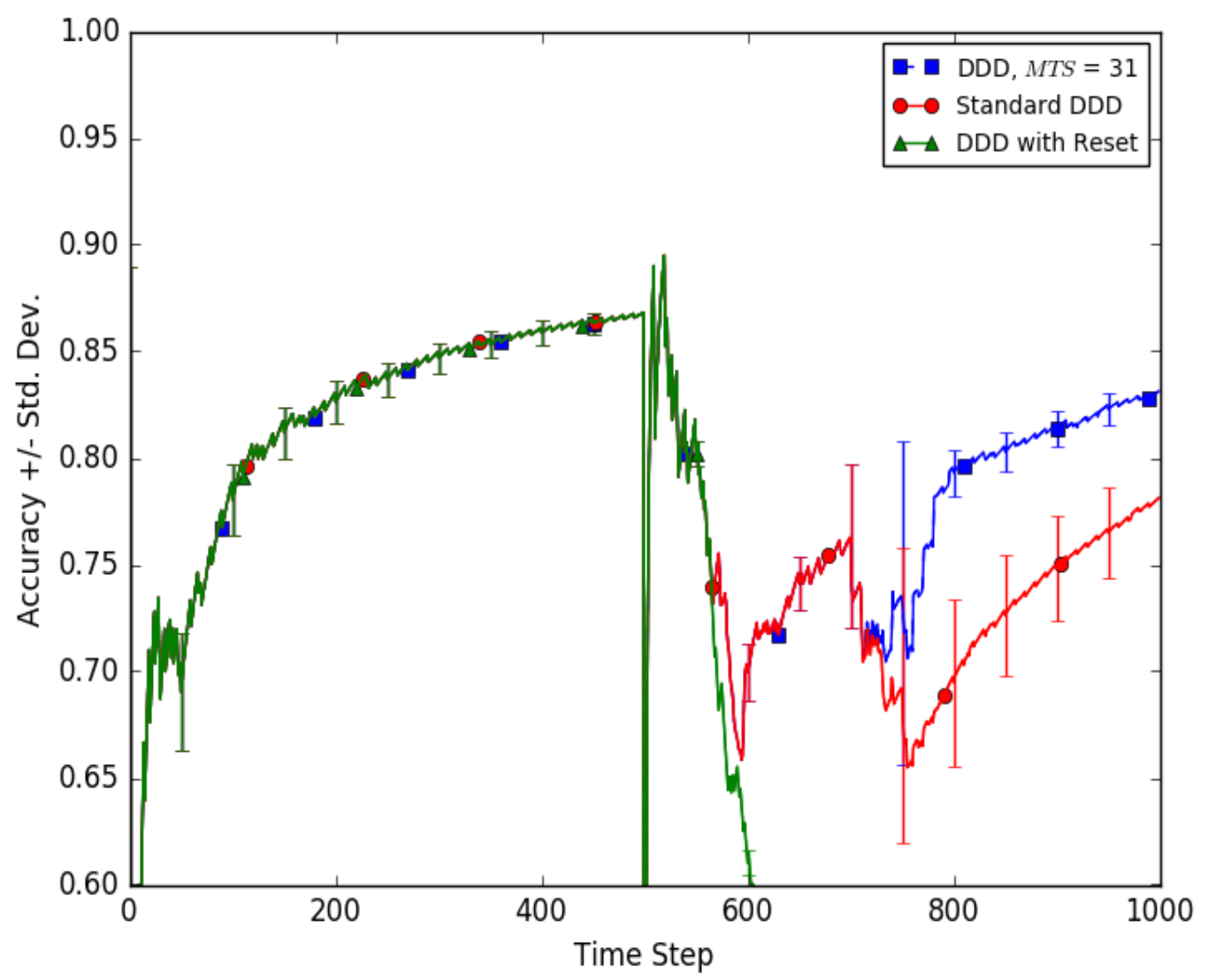

Figure 24. Plane - High Severity/Medium Speed, comparison using MTS 31, Standard DDD, and DDD with Reset.

The graph in Figure 24 shows that Best MTS was significantly better than Standard DDD and DDD with Reset at time steps 750-1000. While the Best MTS sometimes gave higher accuracy than the Standard DDD, in some cases the Standard DDD had better accuracy or the same accuracy as the Best MTS. Figure 25 and Figure 26 give examples of these results. 
Figure 25. Boolean - Medium Severity/High Speed, Comparison of Algorithms

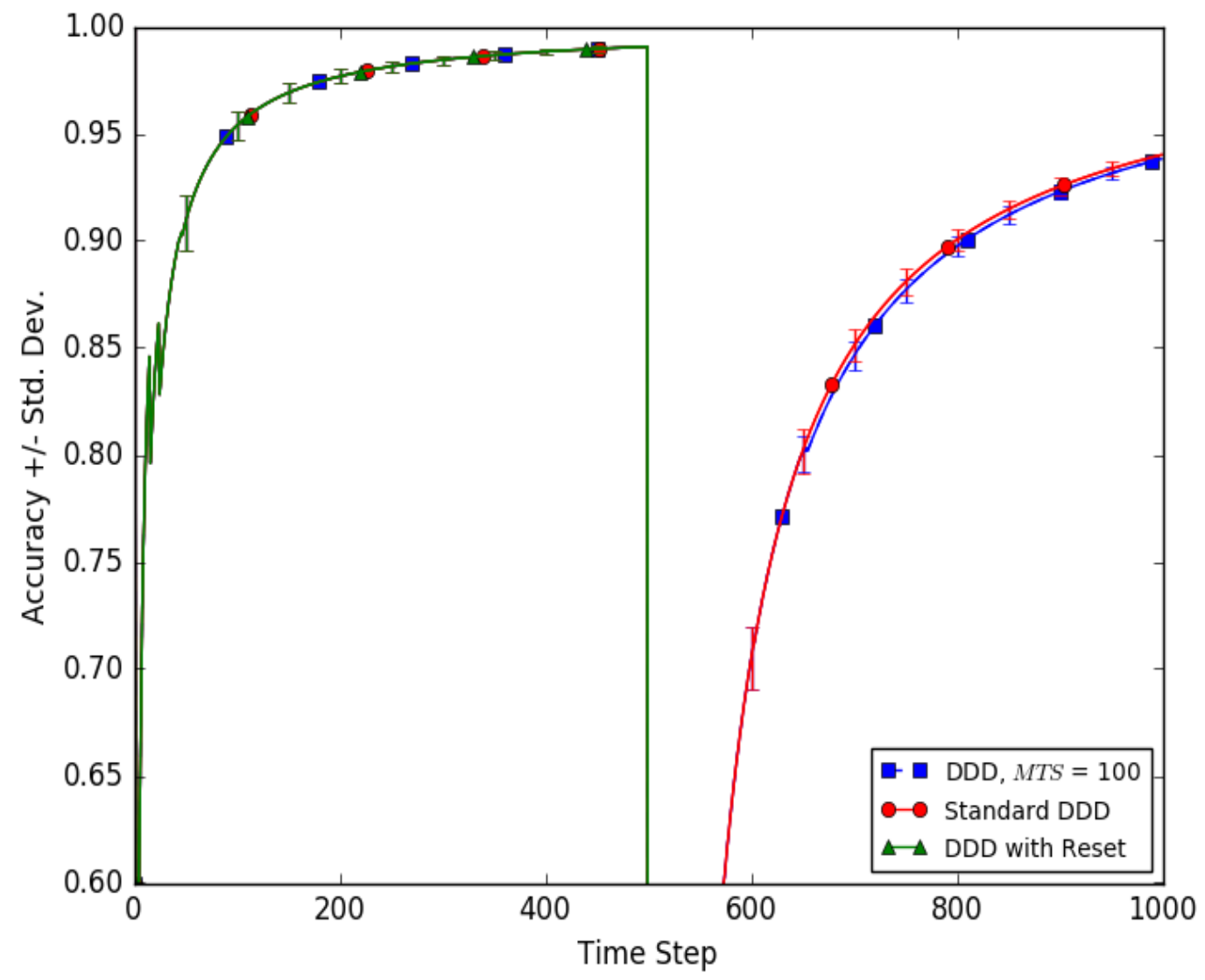

Figure 25. Boolean - Medium Severity/High Speed, comparison using MTS 100, Standard DDD, and DDD with Reset.

As seen in Figure 25, Best MTS and Standard DDD were very close after the concept drift. In this graph Standard DDD provided slightly higher prequential accuracy. 
Figure 26. SineH - Low Severity/Low Speed, Comparison of Algorithms

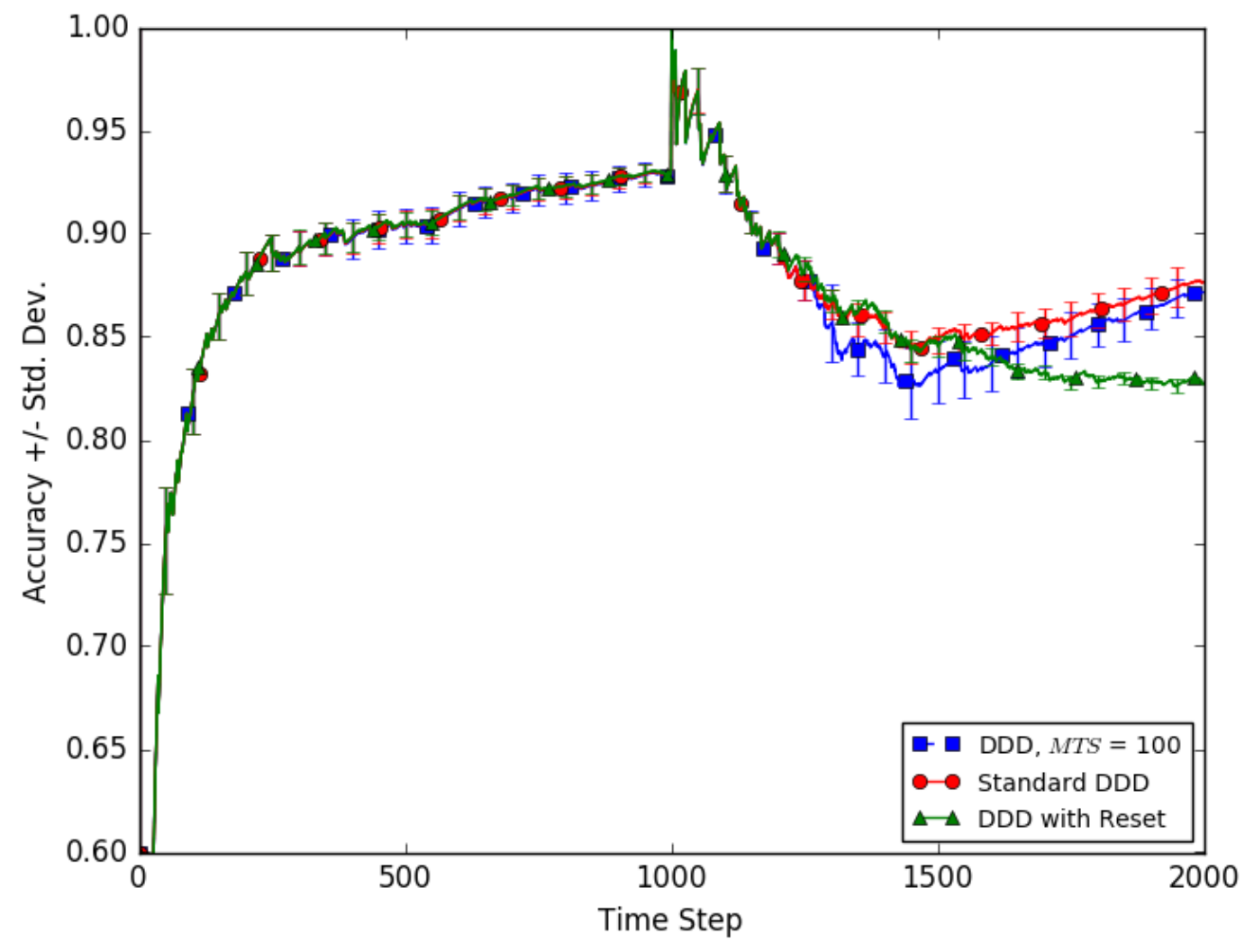

Figure 26. SineH - Low Severity/Low Speed, comparison using MTS 100, Standard DDD, and DDD with Reset.

The graph in Figure 26 shows the Standard DDD produced the highest prequential accuracy from approximately time step 1500 to 2000 .

The results from applying Best MTS, Standard DDD, and DDD with Reset to all artificial datasets are shown graphically in the appendix. A visual inspection of all 54 graphs revealed that Best MTS had the same or higher in prequential accuracy than both Standard DDD and DDD with Reset in $77.78 \%$ (42/54) of the graphs.

Paired $t$-tests were also conducted on the Best MTS and the Standard DDD algorithms on all severity/speed settings at time steps $0.99 N, 1.1 N, 1.5 N$, and $2 N$. As 
stated earlier, these steps were chosen because they occur right before the drift, right after the drift, a little further after the drift, and then much further after the drift. These checkpoints were the same ones used by Minku and Yao (2012). Looking at each of the 54 artificial datasets at the $\alpha=0.05$ level, there was not enough evidence in any case to reject the claim that Best MTS was as good or better than Standard DDD. Using the $t$-test values computed, Best MTS was generally further from the rejection region than was Standard DDD with Circle, SineV, Line, and Plane but was closer to the rejection region using SineH and Boolean.

If Best MTS generally gave higher prequential accuracy than Standard DDD, that is clearly an advantage of Best MTS over Standard DDD. While in some cases the Best MTS algorithm did provide higher accuracy, sometimes Best MTS and Standard DDD were basically equivalent. To help determine the advantage of Best MTS over Standard DDD when accuracies are equivalent, an additional comparison showing the average number of time steps four ensembles were used to predict accuracy for each setting was created. This comparison shows the time steps saved by using the Best MTS algorithm. Figure 27 provides this comparison for the Circle dataset. 
Figure 27. Circle - Comparison of Average Number of Time Steps in Four Ensembles

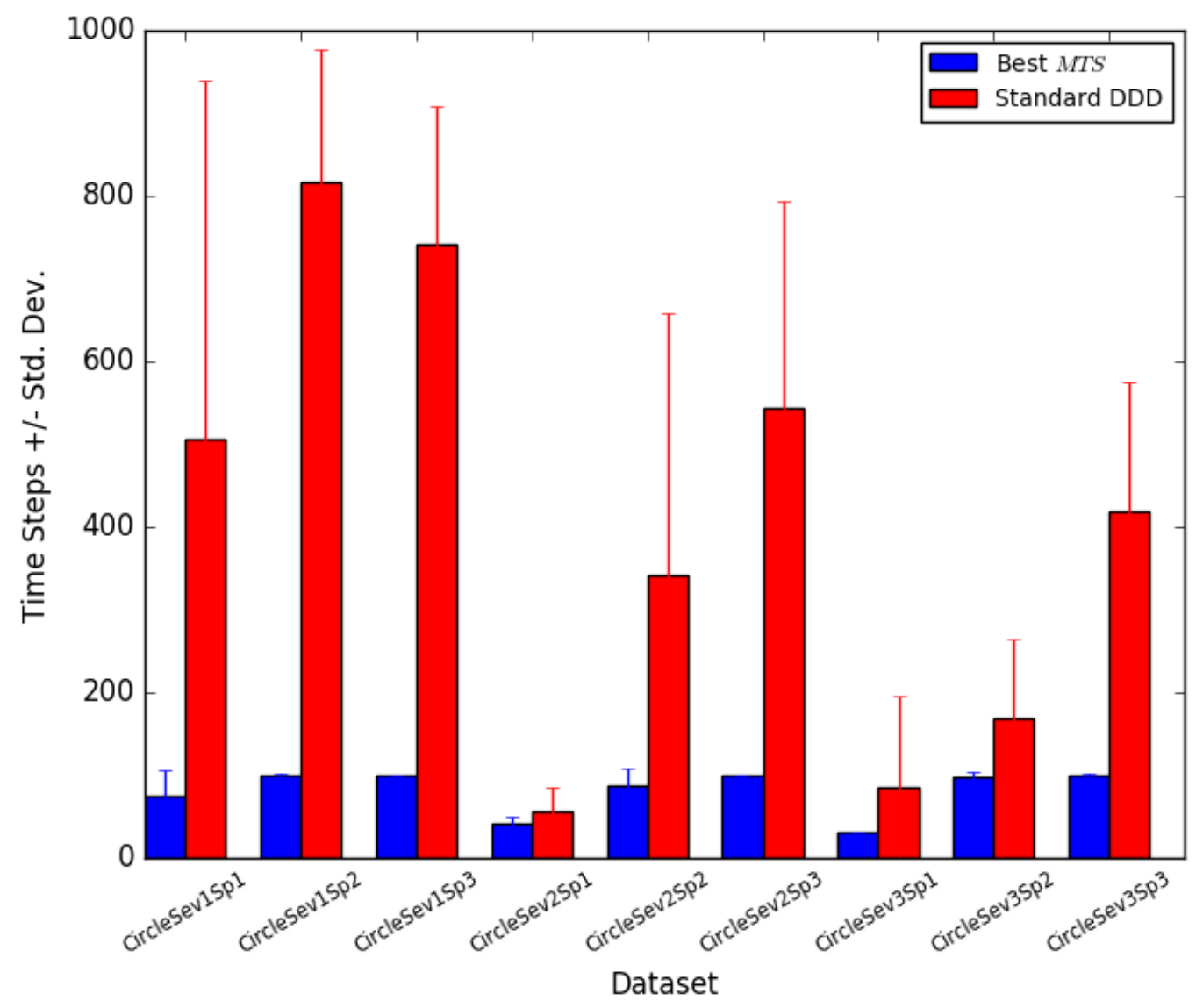

Figure 27. A comparison between the Best MTS and the Standard DDD for the Circle dataset. The graph shows the number of time steps each algorithm used four ensembles to compute prequential accuracy.

Figure 27 shows that the Best MTS algorithm used less time steps in all cases for the Circle dataset. The same graph was created for all artificial datasets and the results in each case showed that Best MTS used four ensembles for less time steps. The comparison graphs for all artificial data is included in the appendix. Following are the same comparisons with the real world data. 
Figure 28. Electricity - Comparison of Algorithms

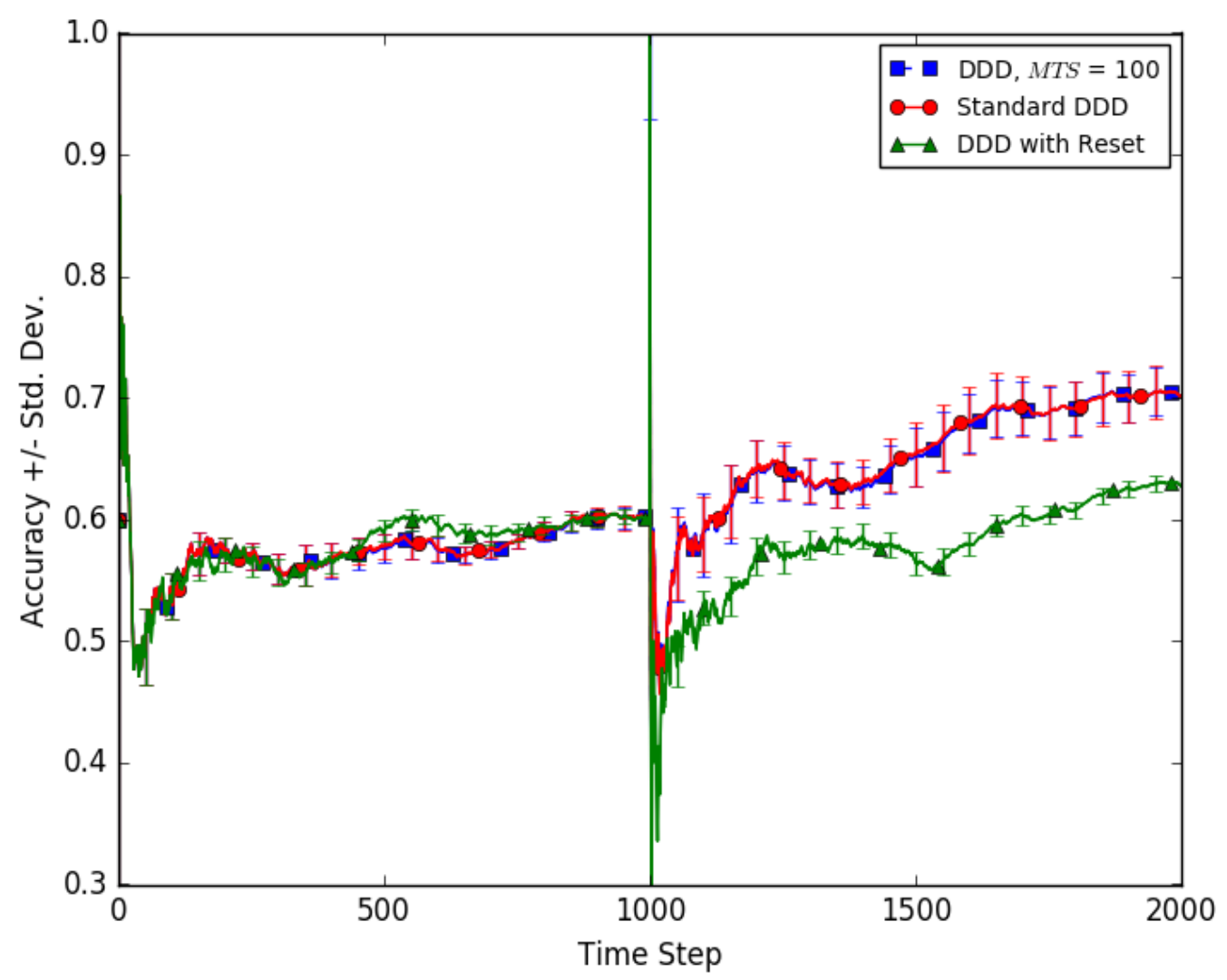

Figure 28. Electricity - comparison using MTS 100, Standard DDD, and DDD with Reset.

Figure 28 shows that with the Electricity dataset, while DDD with Reset is slightly higher before accuracies were reset, Best MTS and Standard DDD were significantly better in the second half of the data. 
Figure 29. Forest CoverType - Comparison of Algorithms

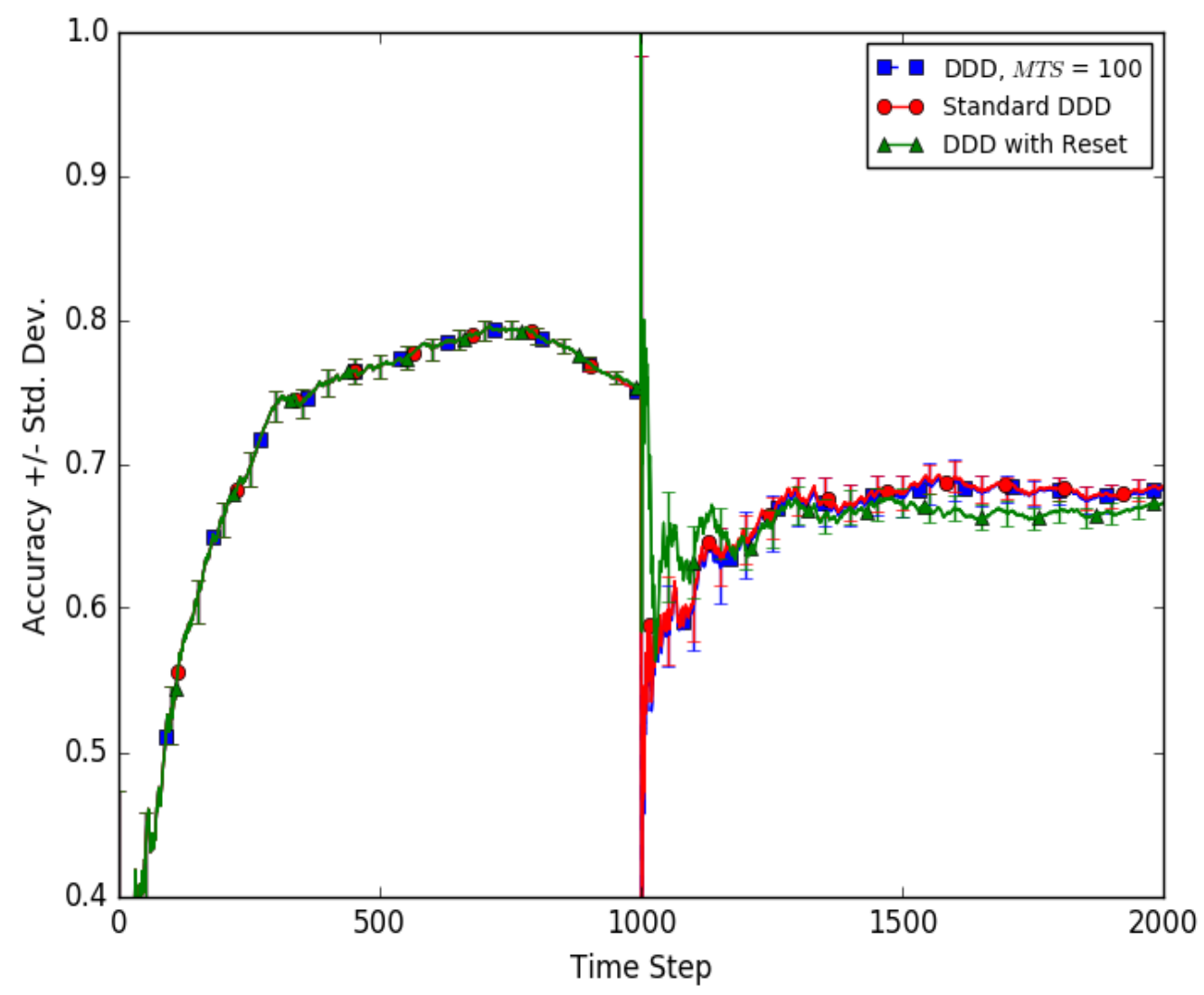

Figure 29. Forest CoverType - comparison using MTS 100, Standard DDD, and DDD with Reset.

With Forest CoverType (in Figure 29) DDD with Reset was somewhat better after accuracies were reset, but Best MTS and Standard DDD were better starting around time step 1500 and continuing to time step 2000. 
Figure 30. KDD 1999 - Comparison of Algorithms

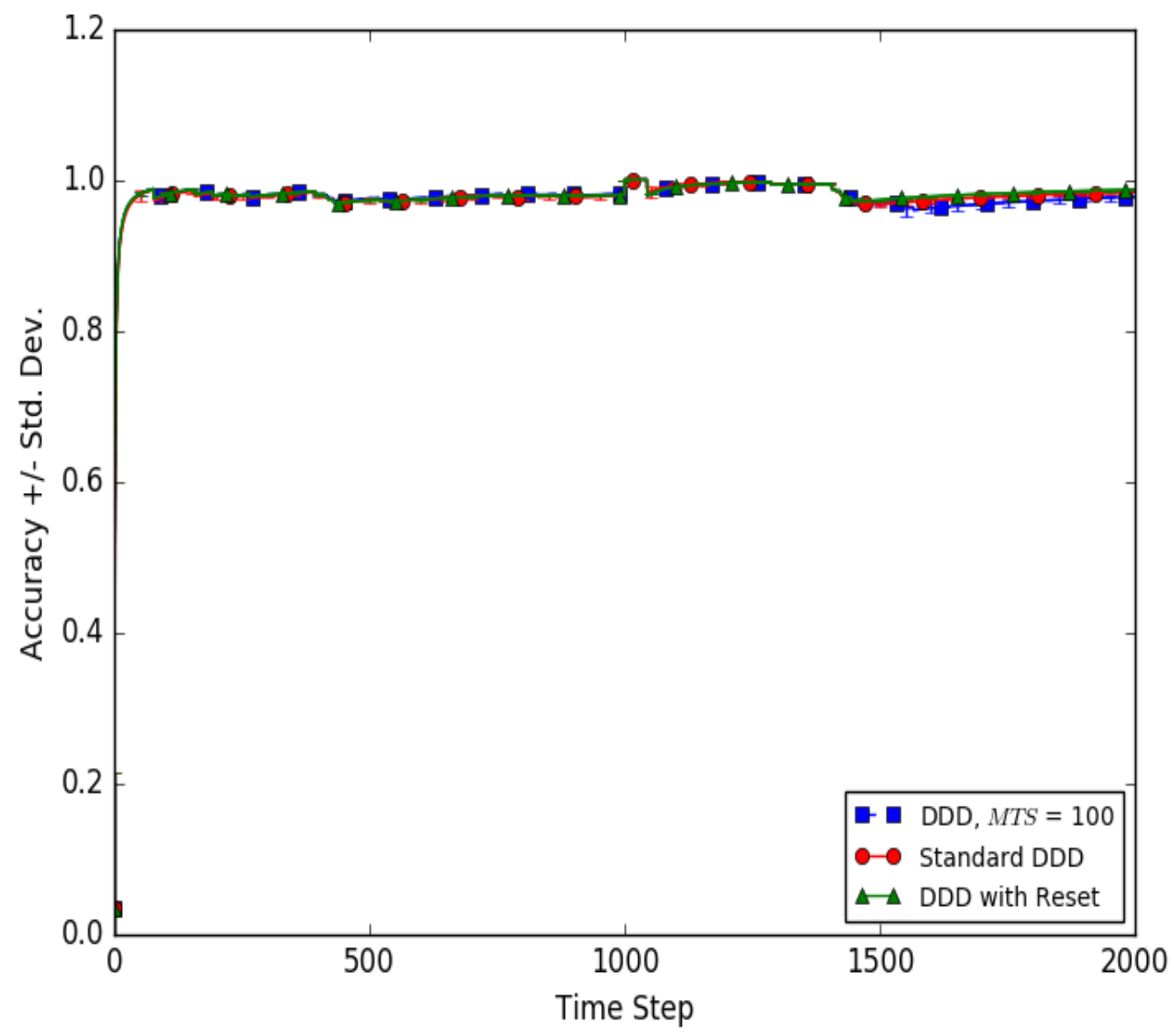

Figure 30. KDD 1999 - comparison using MTS 100, Standard DDD, and DDD with Reset.

In Figure 30, it can be seen that the results with KDD 1999 were very consistent with all three algorithms. Standard DDD and DDD with Reset had very slightly better accuracy from time steps 1500 to 2000 .

Paired $t$-tests were also conducted on the Best MTS and the Standard DDD algorithms on the real world data as was done on the artificial data. The paired $t$-tests were again done at time steps $0.99 N, 1.1 N, 1.5 N$, and $2 N$. These tests showed that there was not enough evidence at the $\alpha=0.05$ level to reject the claim that Best MTS was as good or better than Standard DDD for Electricity and KDD 1999. Interestingly, for the 
Forest CoverType data, there was enough evidence at the $\alpha=0.05$ level to reject the claim that Best MTS was as good or better than Standard DDD using MTS 100 but there was not enough evidence to reject at MTS 31. For the Forest CoverType dataset, MTS 100 was chosen as the Best MTS because it was the best overall. The $t$-test calculation only looked at the data points $0.99 N, 1.1 N, 1.5 N$, and $2 N$.

As was done with the artificial data, an additional comparison was done that gave the average number of time steps four ensembles were used to predict accuracy for each real world dataset. Figure 31 shows the time steps saved by using the Best MTS algorithm. It is clear from this graph that the Best MTS algorithm is more efficient on the number of time steps four ensembles are used in the three real world datasets. 
Figure 31. Real World Data - Average Number of Time Steps in Four Ensembles

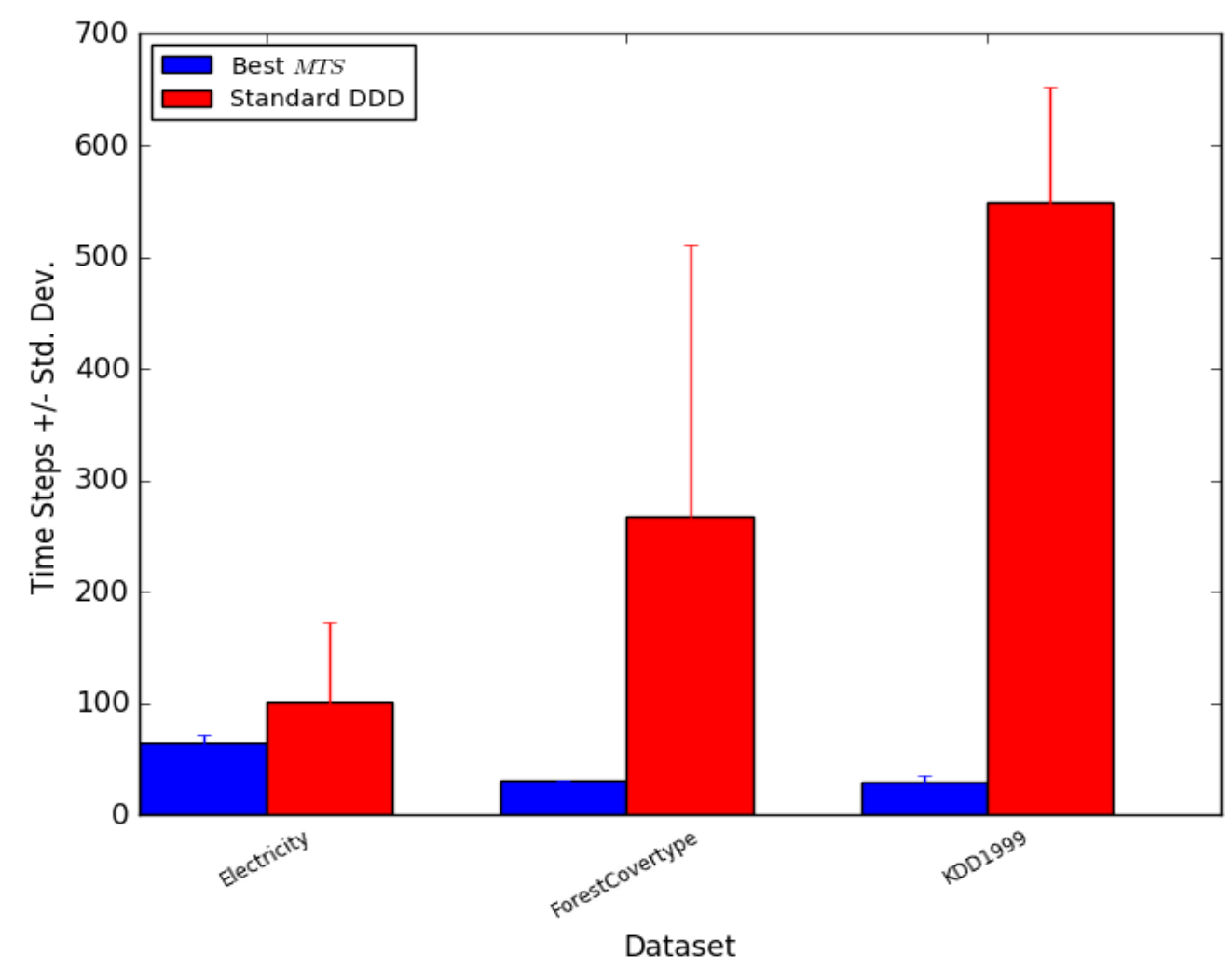

Figure 31. A comparison between the Best MTS and the Standard DDD for the Real World datasets. The graph shows the number of time steps each algorithm uses four ensembles to compute prequential accuracy.

\section{Results of Tests for False Positive Concept Drift}

As stated in Chapter 3, the $W$ parameter is used in the DDD algorithm as a weight on the old low diversity ensemble to help keep the algorithm from reacting to what appears to be concept drift but is not. The impact of changes in $W$ related to settings in the MTS parameter was explored. Following Minku and Yao (2012), additional testing was done by forcing false positive concept drift at specific times with the artificial data and the real world data. With the artificial datasets, false concept drift was forced halfway through the first concept and halfway through the second concept. With the real world 
data, false positive concept drift was created on the first quarter, halfway, and in the third quarter of the data. The best MTS parameter found in earlier testing was used with different $W$ parameters and was compared to different $W$ parameters using the Standard DDD. Note that the MTS parameter can be different for each dataset. The best MTS parameter ran 30 times using $(W=1, W=2$, and $W=3)$ to test the impact on prequential accuracy for each of these settings. Figure 32 shows the impact of changes in $W$ when false positive drift was encountered in the Circle Low Severity, High Speed dataset. For the artificial datasets, the same four Circle datasets will be shown with changes in the $W$ parameter, followed by interesting results using the artificial data. Following the artificial data will be the real world data. 
Figure 32. Circle - Low Severity/High Speed, Comparison of $W$ values

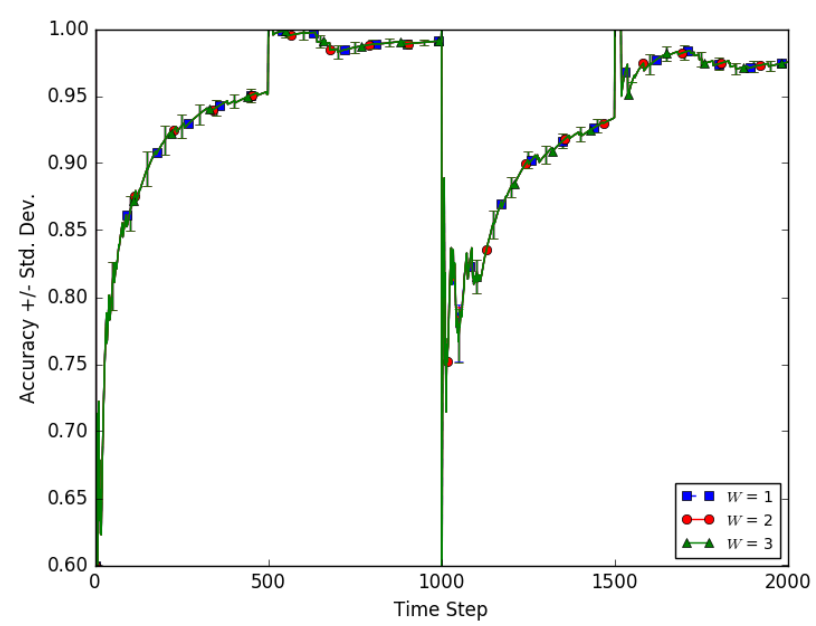

a. Best MTS, 31

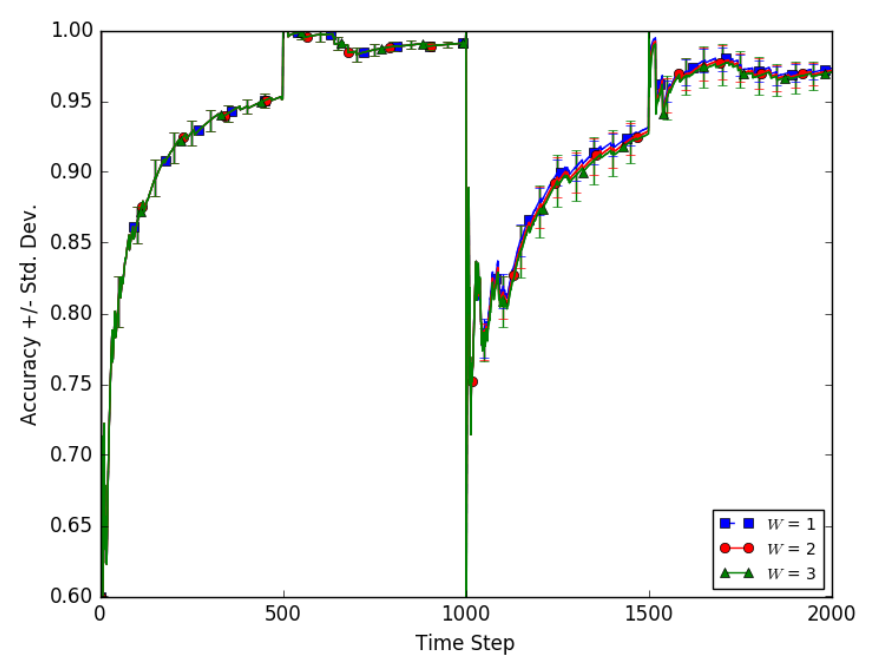

b. Standard DDD

Figure 32. A comparison of $W$ results for (a) the Best MTS, 31 and (b) the Standard DDD.

It can be seen that the impact of changes in the $W$ parameter were minor for Best MTS and Standard DDD. The false positives at 500 and 1500 seem to be handled well regardless of the setting for $W$. Following is Figure 33 using Circle Low Severity, Low Speed. 
In Figure 33, while Best MTS was consistent for all $W$ values, changes in the $W$ values for the Standard DDD showed improved accuracy for $W=1$ followed by $W=2$ after time step 1500 .

Figure 33. Circle - Low Severity/Low Speed, Comparison of $W$ values

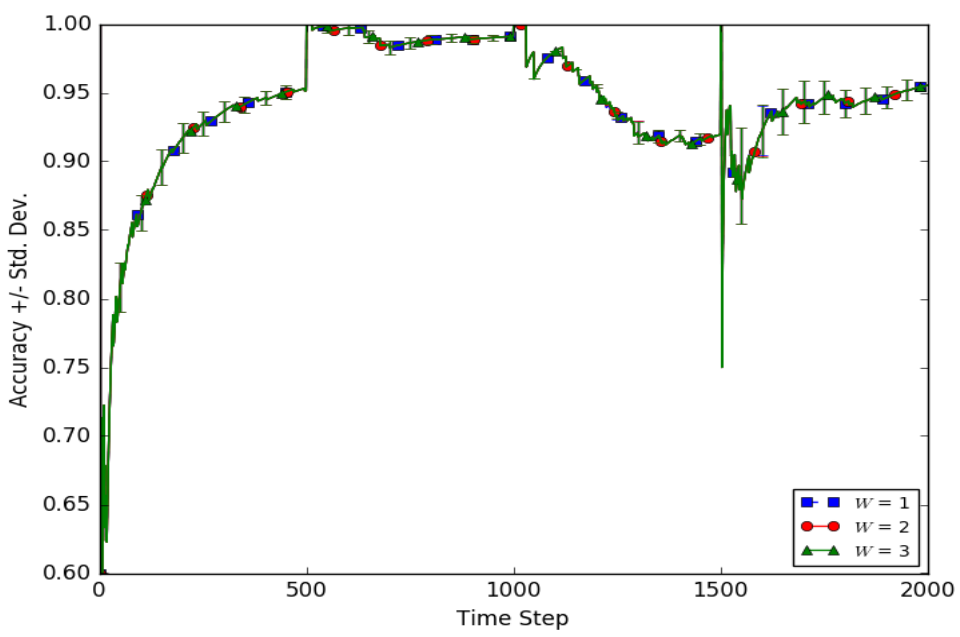

a. Best MTS, 100

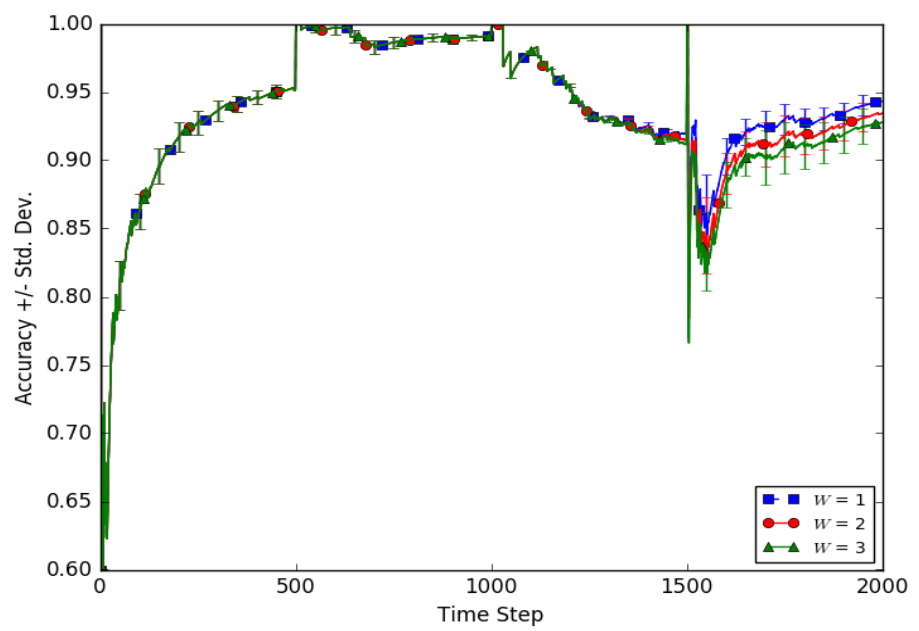

b. Standard DDD

Figure 33. A comparison of $W$ results for (a) the Best MTS, 100 and (b) the Standard DDD. 
Figure 34. Circle - High Severity/High Speed, Comparison of $W$ values

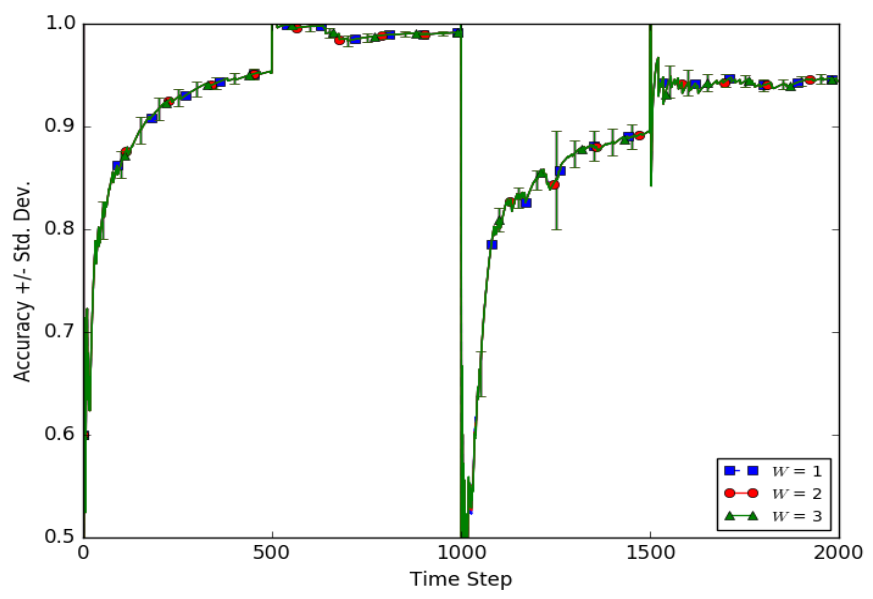

a. Best MTS, 31

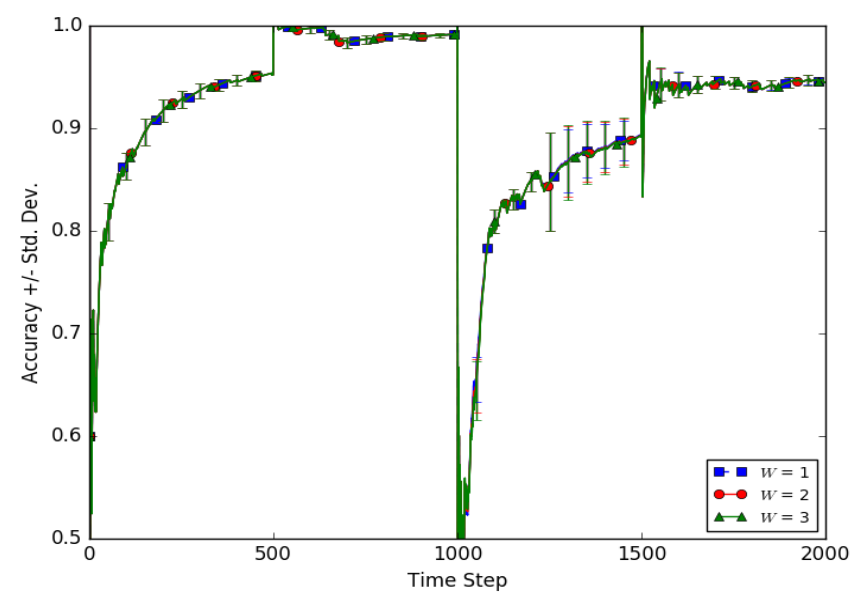

b. Standard DDD

Figure 34. A comparison of $W$ results for (a) the Best MTS, 31 and (b) the Standard DDD.

In Figure 34, the $W$ settings were very similar for Best MTS and Standard DDD. False positives seem to be handled consistently regardless of $W$ setting. 
Figure 35. Circle - High Severity/Low Speed, Comparison of $W$ values

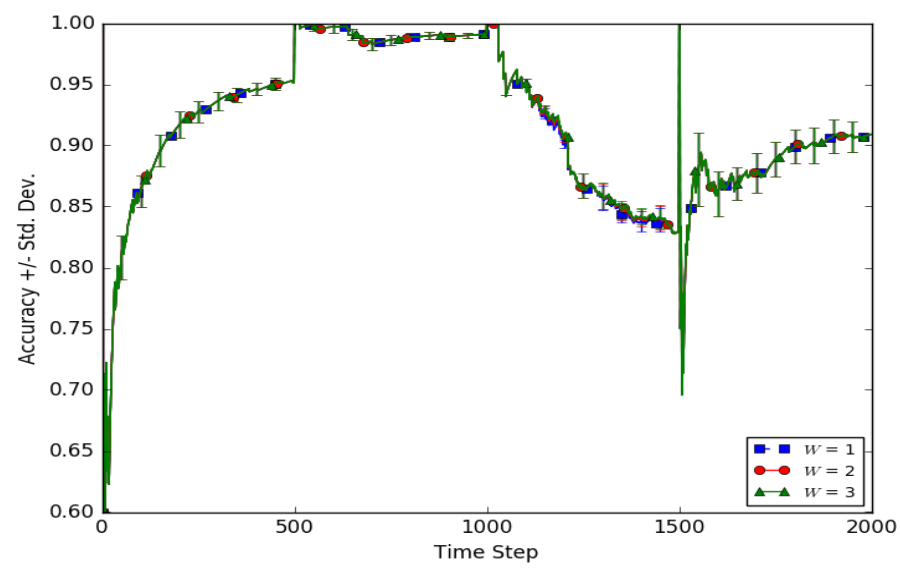

a. Best MTS, 100

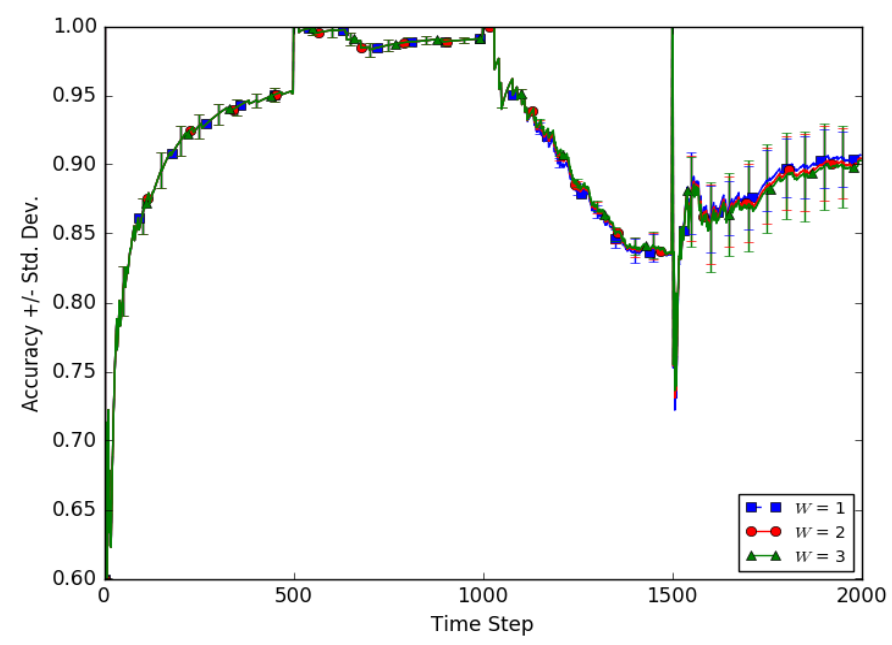

b. Standard DDD

Figure 35. A comparison of $\mathrm{W}$ results for (a) the Best MTS, 100 and (b) the Standard DDD.

In Figure 35, it can be seen again that false positives were handled well for all three settings of $W$. In Best $M T S$, the $W=3$ provided slightly higher accuracy at time steps 1100-1200 and 1400-1500. The setting $W=1$ was slightly better after the false positive at 
time step 1500 for the Standard DDD. Following are additional comparisons using the same settings for $W$ for Best MTS and Standard DDD.

Figure 36. SineV - Medium Severity/Low Speed, Comparison of $W$ values

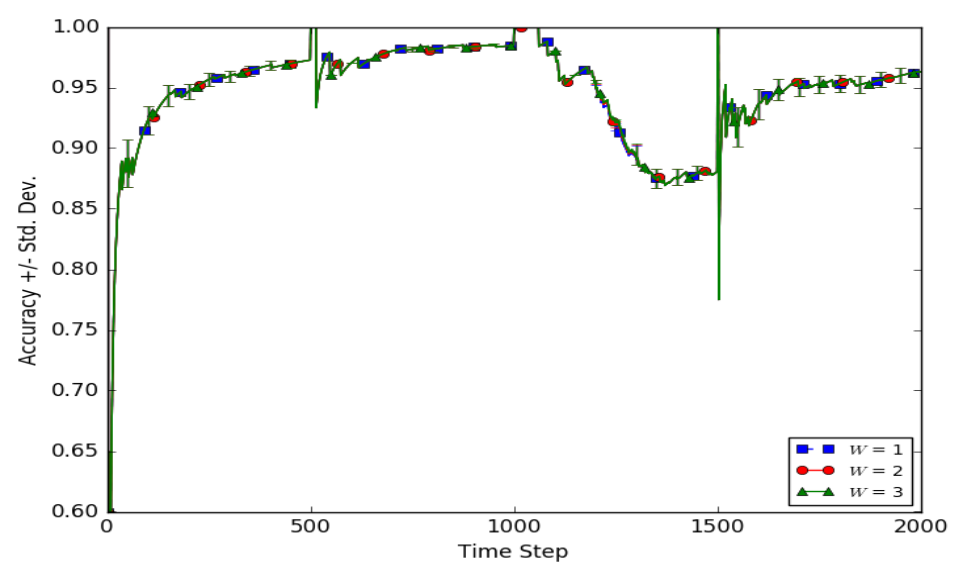

a. Best MTS, 100

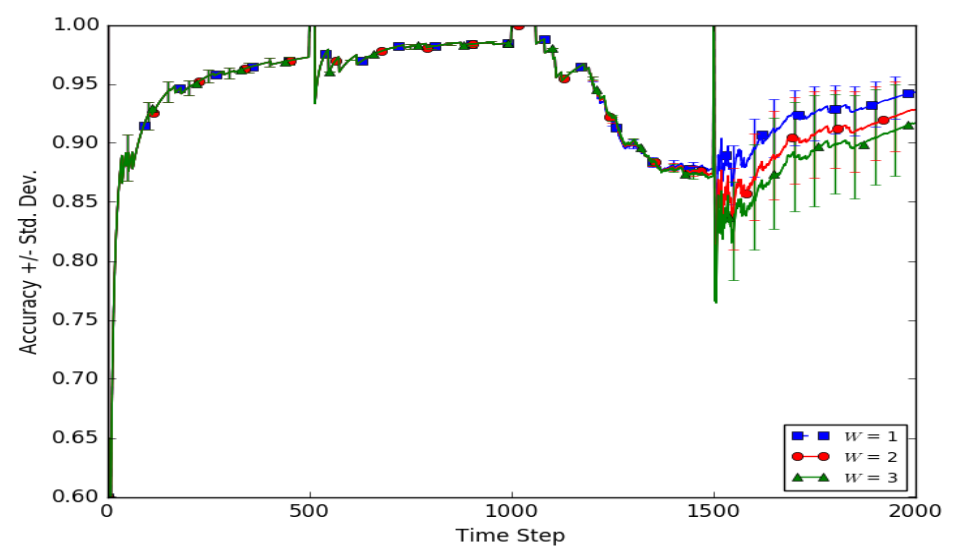

b. Standard DDD

Figure 36. A comparison of $\mathrm{W}$ results for (a) the Best MTS, 100 and (b) the Standard DDD.

While Figure 36 had consistent accuracies across $W$ settings for Best MTS, Standard DDD showed improved accuracy for $W=1$ and then $W=2$ after the false positive concept drift at time step 1500. 
Figure 37. SineH - Low Severity/High Speed, Comparison of $W$ values

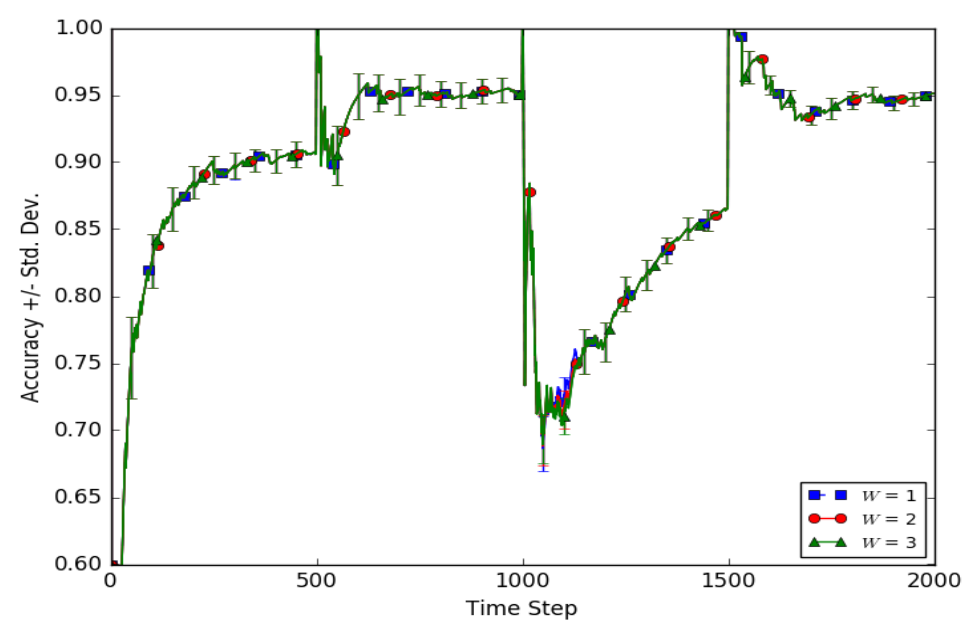

a. Best MTS, 100

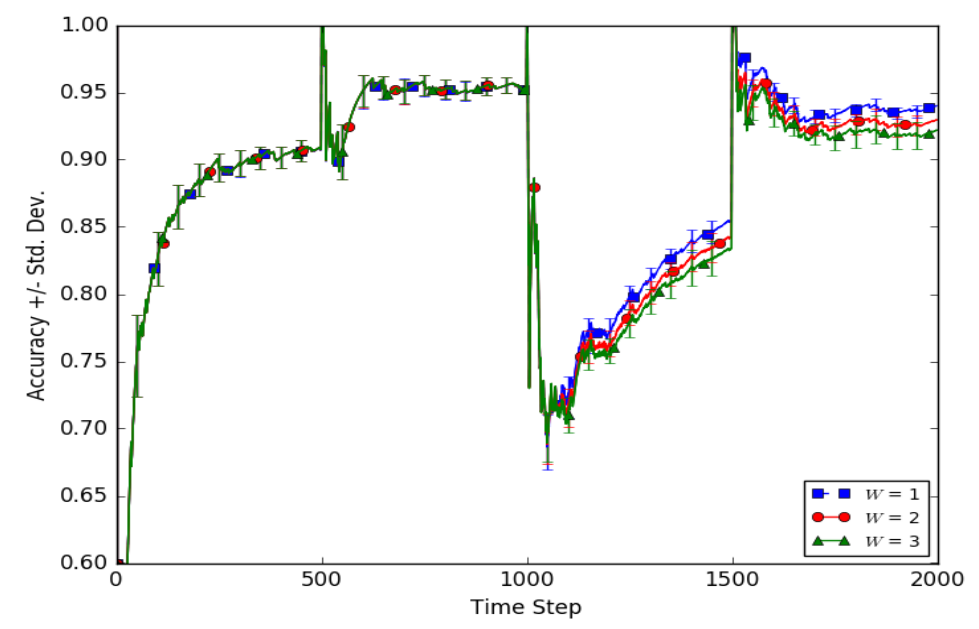

b. Standard DDD

Figure 37. A comparison of $W$ results for (a) the Best MTS, 100 and (b) the Standard DDD.

In Figure 37, while the Best MTS settings were the same for each $W$ value used, results from the Standard DDD showed improved accuracy from $W=1$ at both the actual concept drift around 1000 and the false concept drift at 1500. 


\section{Figure 38. SineH - Medium Severity/Low Speed, Comparison of $W$ values}

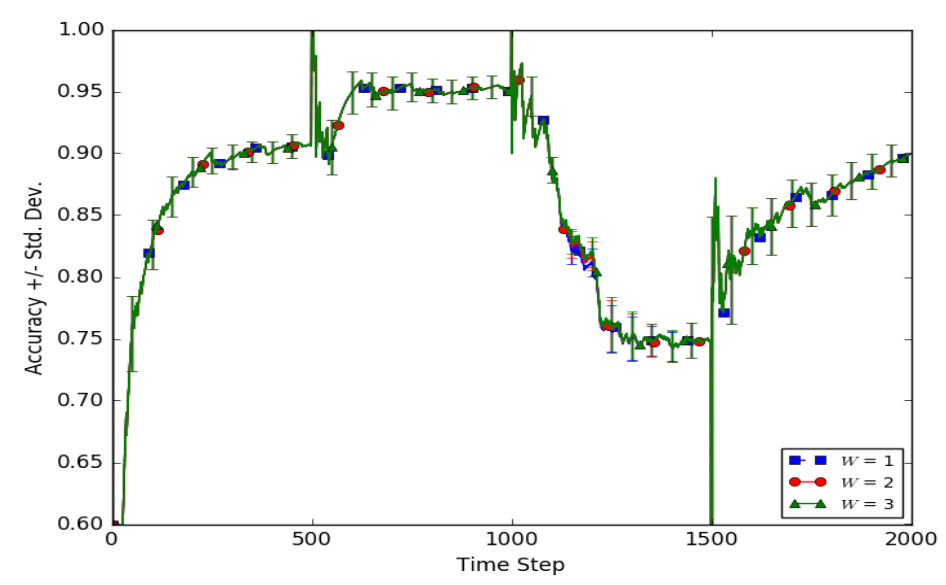

a. Best MTS, 100

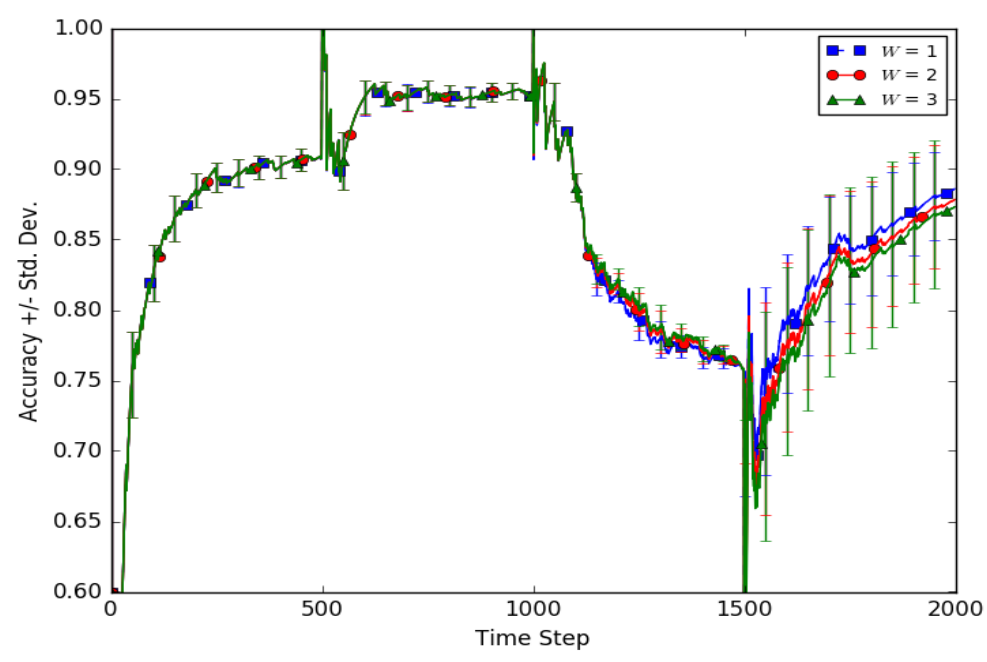

b. Standard DDD

Figure 38. A comparison of $W$ results for (a) the Best MTS, 100 and (b) the Standard DDD.

Figure 38 shows that $W=3$ produced slightly better accuracy time steps 1100 to 1200 for Best $M T S$. For Standard DDD, $W=3$ gave the best accuracy from time steps 1100 to 1500 , while the best accuracy from time steps 1500 to 2000 was given by $W=1$. 
Figure 39. Line - Medium Severity/High Speed, Comparison of $W$ values

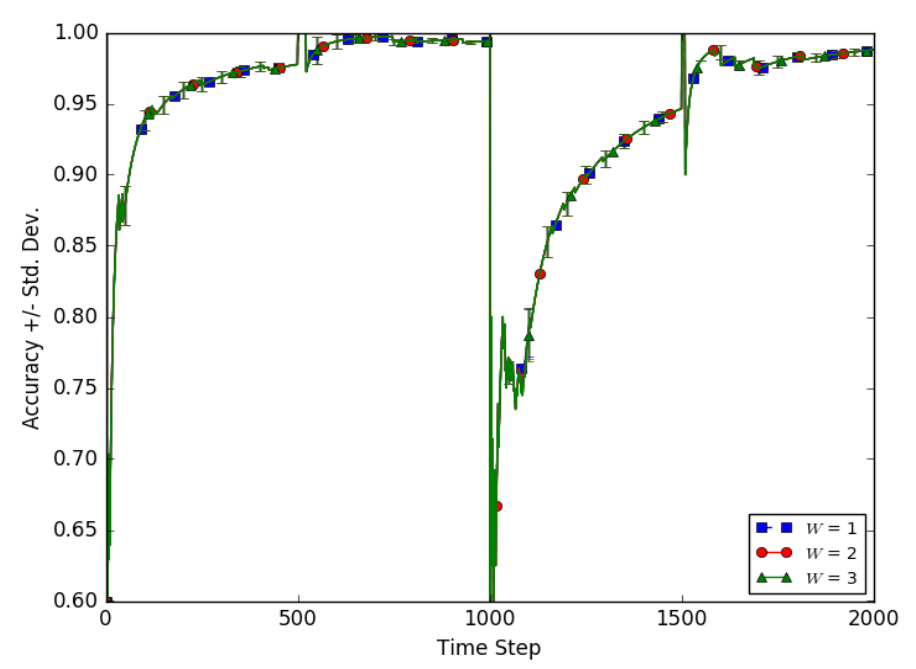

a. Best MTS, 100

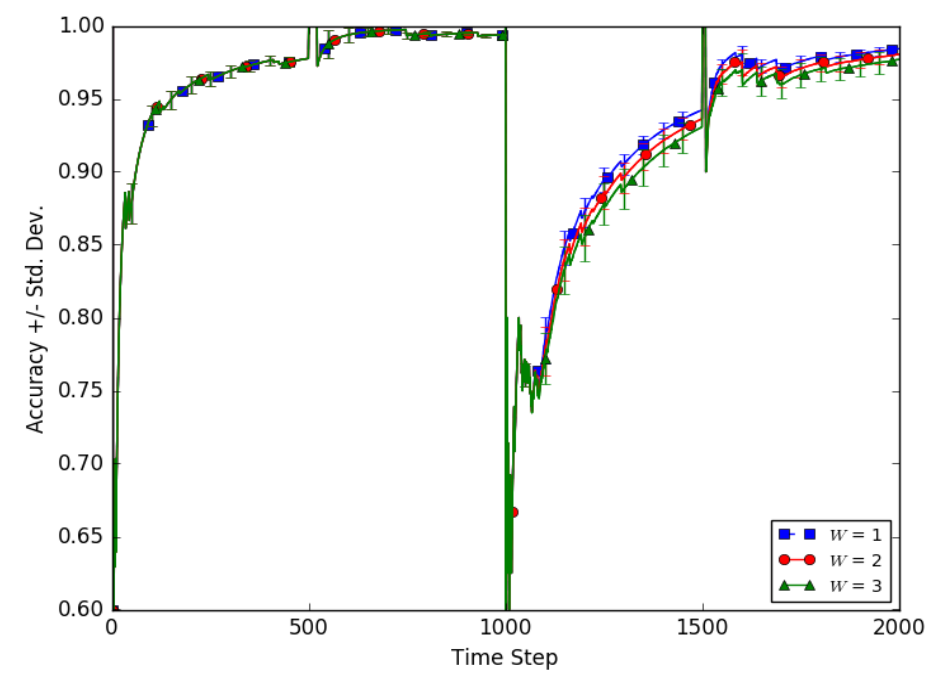

b. Standard DDD

Figure 39. A comparison of $W$ results for (a) the Best MTS, 100 and (b) the Standard DDD.

The $W$ settings were similar for Best $M T S$ in Figure 39. With Standard DDD, $W=1$ followed by $W=2$ gave the highest accuracy from approximately time step 1200 to 2000 . 
Figure 40. Plane - High Severity/Medium Speed, Comparison of $W$ values

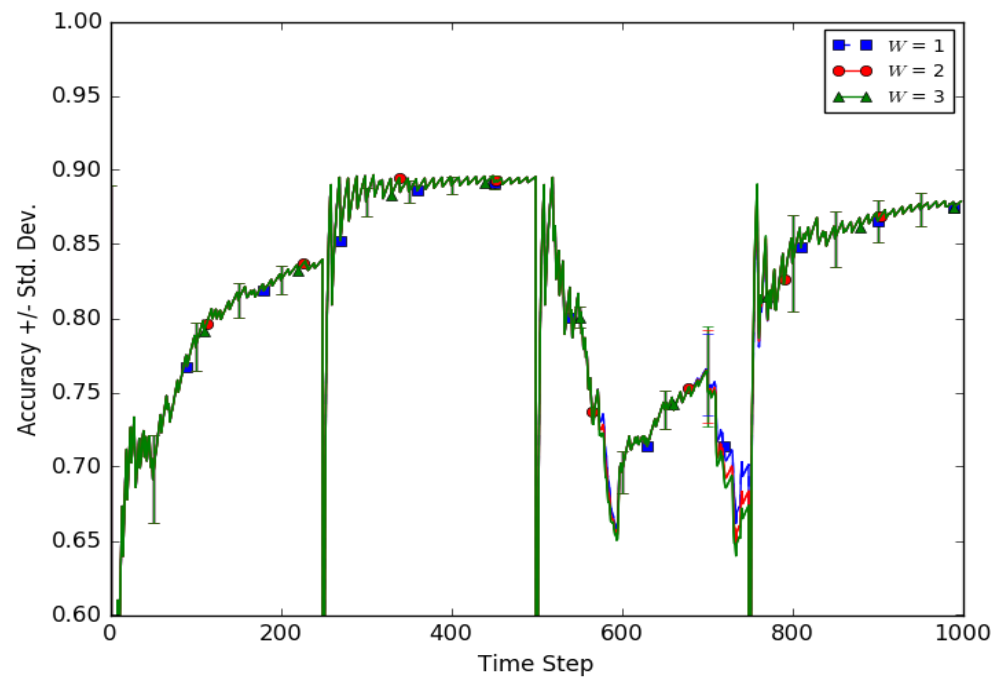

a. Best MTS, 31

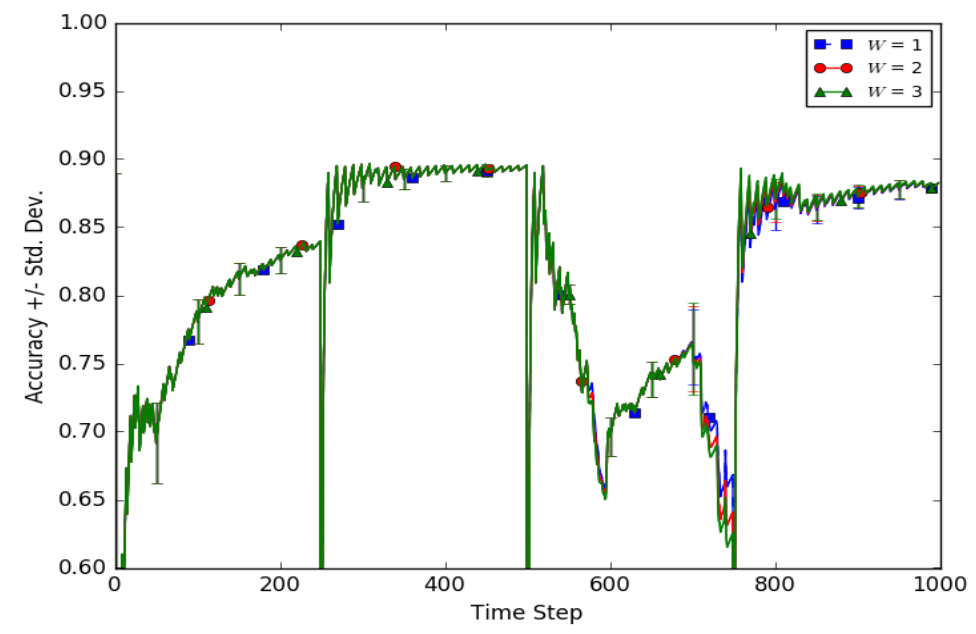

b. Standard DDD

Figure 40. A comparison of $W$ results for (a) the Best MTS, 31 and (b) the Standard DDD.

Figure 40 shows that $W=1$ gave the highest accuracy right before time step 750 (a false concept drift) for both Best MTS and Standard DDD. From time step 750 to 1000 the results are the same for Best MTS but for Standard DDD $W=3$ has the highest prequential accuracy. Following are the results of the tests with the real world datasets. 
Figure 41. Electricity - Comparison of $\boldsymbol{W}$ values

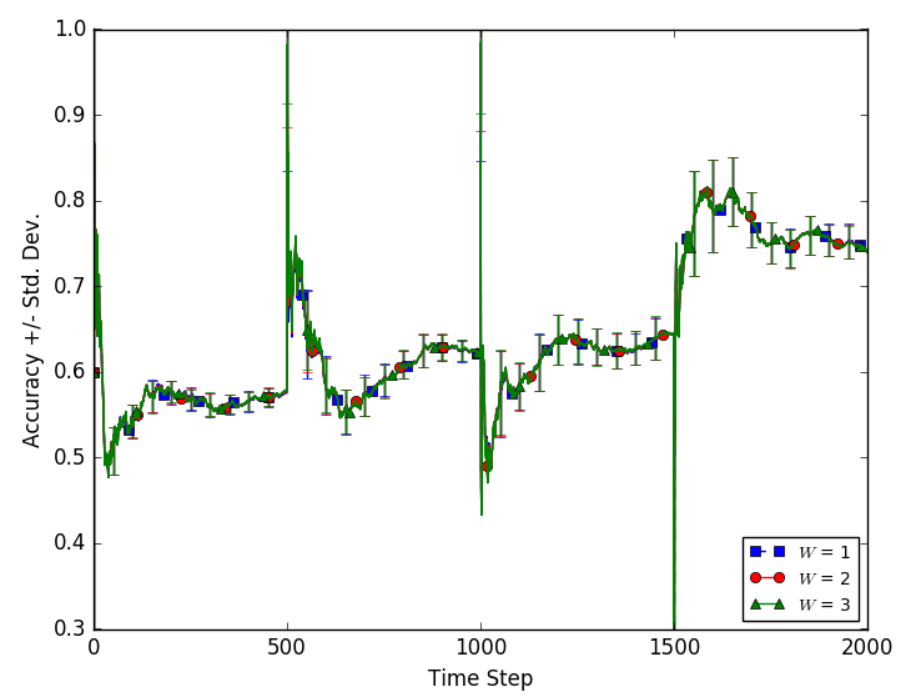

a. Best MTS, 100

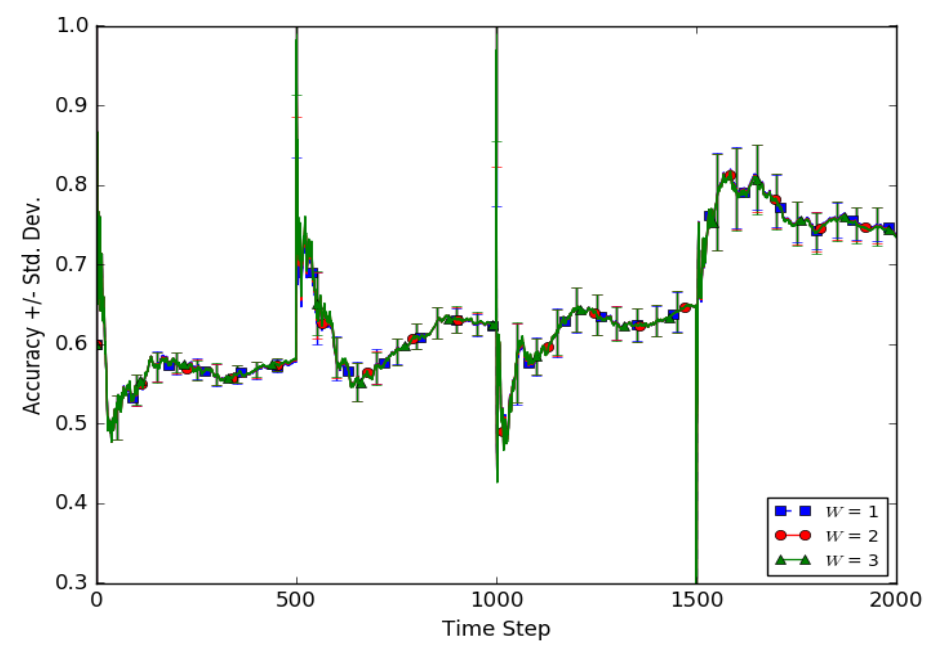

b. Standard DDD

Figure 41. A comparison of $W$ results for (a) the Best MTS, 100 and (b) the Standard DDD.

As can be seen in Figure 41, changing the $W$ value did not have an impact on accuracy on Best MTS or on Standard DDD in Electricity, even when false concept drift occurred. Following is the Forest CoverType dataset. 
Figure 42. Forest CoverType - Comparison of $W$ values

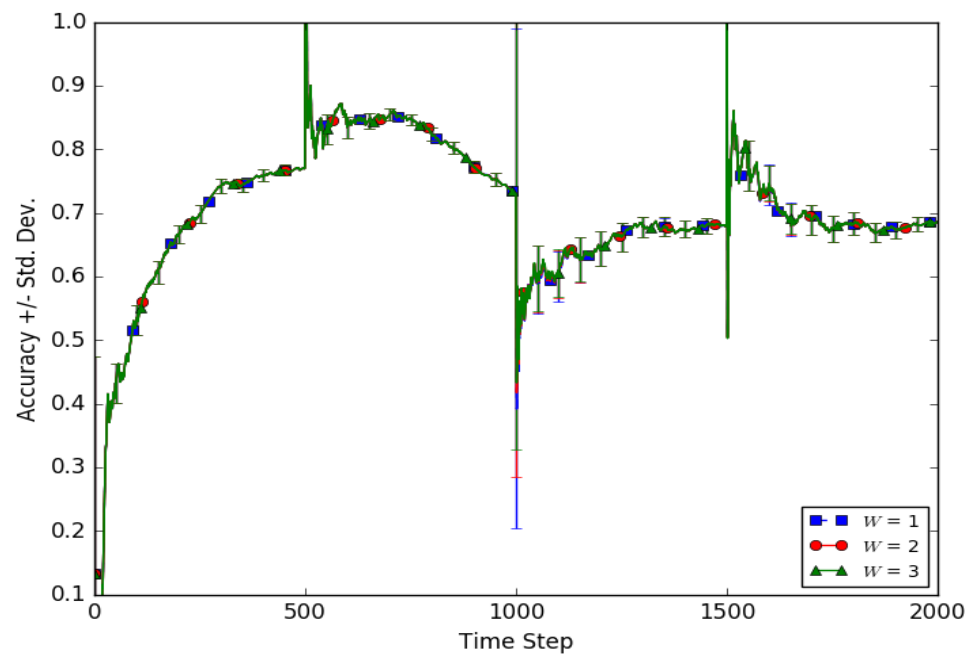

a. Best MTS, 100

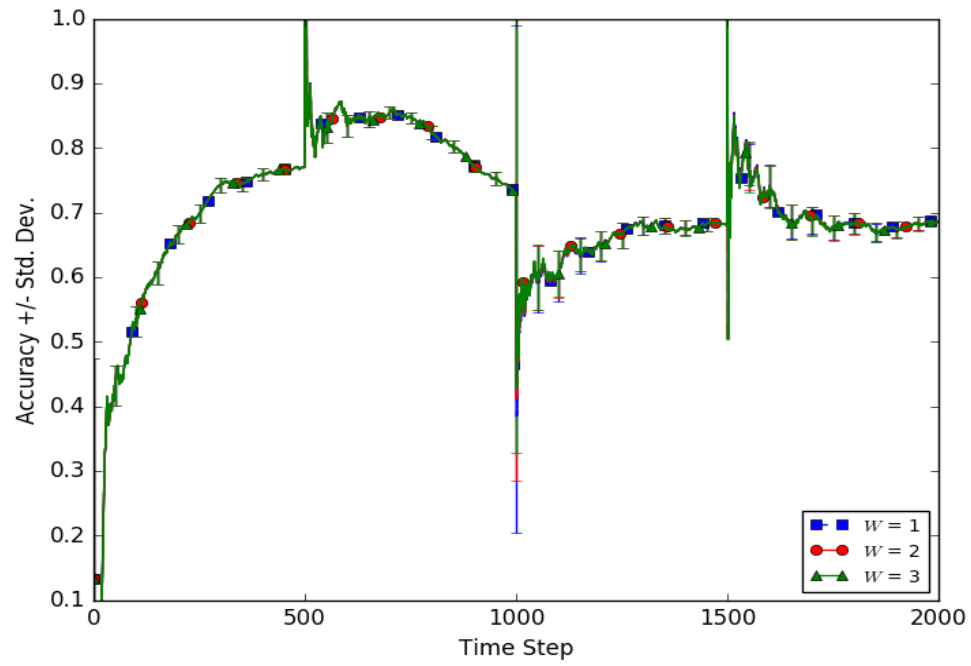

b. Standard DDD

Figure 42. A comparison of $W$ results for (a) the Best MTS, 100 and (b) the Standard DDD.

Again, in Figure 42, changing the $W$ value in Forest CoverType dataset did not have an impact on accuracy for Best MTS or on Standard DDD, even when false concept drift occurred. 
Figure 43. KDD 1999 - Comparison of $W$ values

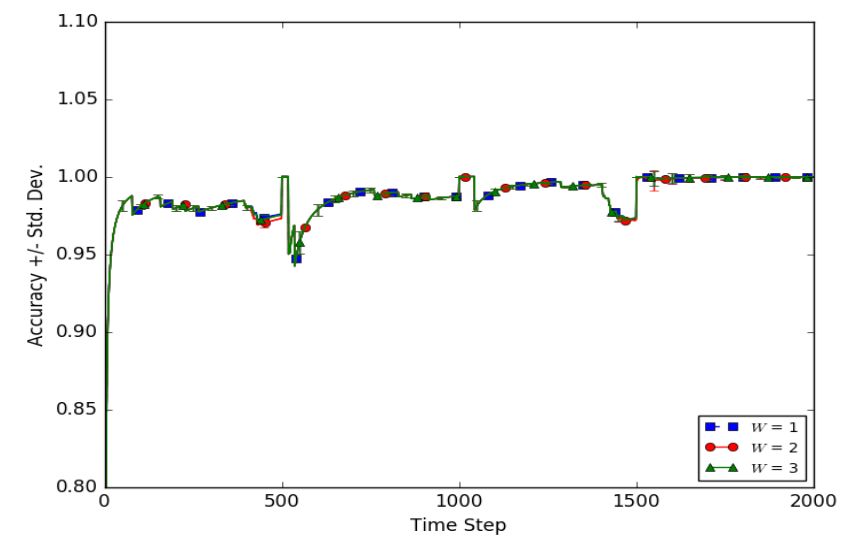

a. Best MTS, 100

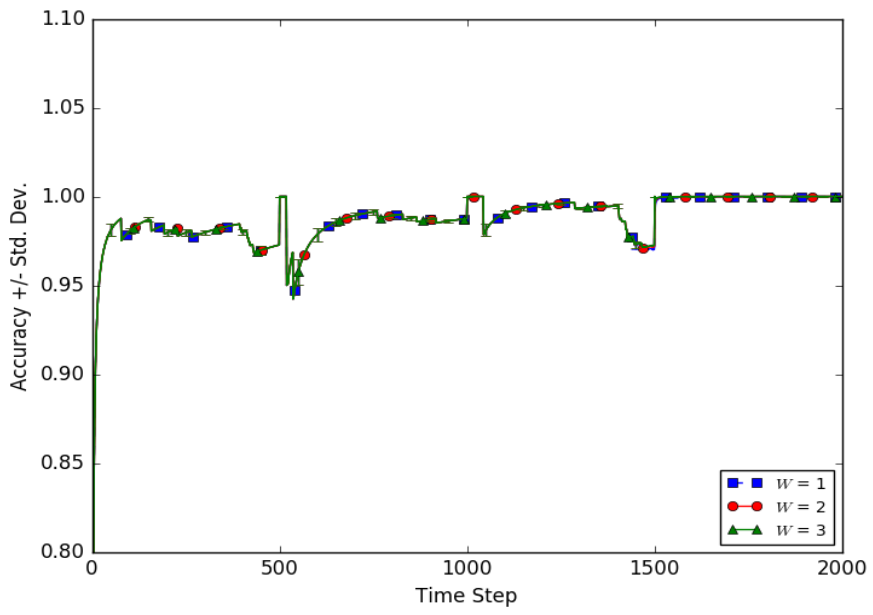

b. Standard DDD

Figure 43. A comparison of $W$ results for (a) the Best MTS, 100 and (b) the Standard DDD.

With KDD 1999 in Figure 43, all W settings were very close with Best MTS, except right before time step 500 . Here both $W=1$ and $W=3$ gave slightly better accuracy than $W=2$. All $W$ settings for Standard DDD gave similar accuracies. 
One goal of checking the $W$ settings was to see how well the Best MTS algorithm performed when false positive concept drift was present. While increasing $W$ did seem to help improve prequential accuracy when false positive concept drift occurred in a few cases, it was clear that the accuracy of Best MTS did not suffer greatly when false positive concept drift occurred, regardless of $W$ setting.

\section{Summary of Results}

The results of the study of the impact of using MTS were shown in this chapter. Artificial and real world datasets were used to select the Best MTS for each dataset and compare this MTS to the Standard DDD and to DDD with Reset. A comparison of number of time steps four ensembles where used to compute prequential accuracy was also provided. Statistical $t$-tests were also used to compare the Best MTS and the Standard MTS at points $0.99 N, 1.1 N, 1.5 N$, and $2.0 N$. A comparison was also done showing the impact of changing the $W$ parameter when false positive concept drift was introduced.

The results from this study clearly showed that Best MTS provides equal or improved prequential accuracy over Standard DDD and DDD with Reset. In a direct comparison with Standard DDD, Best $M T S$ was also able to provide comparable prequential accuracy in fewer time steps. Using fewer time steps is an advantage of Best MTS because here four ensembles are used a shorter amount of time, providing time and space savings. 


\section{Chapter 5}

\section{Conclusions, Recommendations, and Summary}

\section{Conclusions}

Based on the results of the tests completed on artificial and real world datasets, it is clear that in many cases the number of time steps four ensembles are in memory can be reduced without sacrificing prequential accuracy. The use of the Maximum Time Step (MTS) parameter with the DDD algorithm gave comparable and sometimes better average prequential accuracy with less iterations. Regarding alternative explanations of results, it was noted that $t$-test values tended to fall closer to the rejection region as the speed of the concept drift decreased. One explanation for this is that while the test files used had increasing concept drift severity, the concept drift speed remained the same, using the test data from Minku and Yao (2012).

Additional tests were conducted by changing the weight $(W)$ in the old low diversity ensemble to keep the Best MTS algorithm from reacting to what appeared to be concept drift but was not. The same settings on the $W$ parameter were used with the Standard DDD algorithm for comparison. One goal of checking the $W$ settings was to see how well the Best MTS algorithm performed when false positive concept drift was present. While increasing $W$ did seem to help produce improved accuracy when false positive concept drift occurred in a few cases, it was clear that the accuracy of Best MTS did not suffer greatly when false positive concept drift occurred, regardless of $W$ setting. 


\section{Recommendations}

Since it appears that the use of the MTS parameter in the DDD algorithm gives equivalent or higher prequential accuracy with less iterations, the addition of the MTS parameter should be seen as an improvement on the DDD algorithm without the MTS parameter. Since the best MTS was not the same for each dataset tested and since four specific values for the MTS were chosen for testing, it seems future research could focus on techniques to dynamically determine the MTS to use, based on the severity and speed of the concept drift. In several cases the accuracies produced by the different MTS settings were very close. For example, with the Line dataset using Medium Severity and Low Speed, the prequential accuracy of MTS 75 was just slightly less than MTS 100. MTS 100 was selected as the best MTS but it did raise the question of how to select the MTS for a specific dataset that would give the best results and would balance the tradeoff between accuracy and number of ensembles used. Further research could be done to select an MTS prior to the DDD with MTS algorithm. In this dissertation the best MTS was selected based on tests using artificial and real world datasets. A more dynamic approach would be to select the $M T S$ based on stored characteristics of similar datasets. Perhaps artificial intelligence techniques could aid in this process.

Another potential area for future research relates to the number of ensembles used during a concept drift. The Best MTS and Standard DDD algorithms both use four ensembles during concept drift. A topic for future research could be to test the Best MTS and Standard DDD algorithms with more than four ensembles. An increase in the use of distributed processing would make this approach practical. 


\section{Summary}

An increasing amount of data comes in the form of data streams. Typically, a data stream is continuous and normally processed only once. A common problem with data streams is that the underlying concept we are trying to learn can be constantly evolving. This dissertation built upon the DDD algorithm implemented by Minku and Yao (2012). Their method used multiple ensembles, each consisting of online classifiers; the predicted class is the mode class or a weighted average of the constituent classifiers in the ensembles. Using a set of diverse classifiers with different strengths and weaknesses resulted in the classification accuracy of the ensembles to be significantly greater than any of its constituent classifiers. Since concept drift can have different speeds and severities, it was found that multiple ensembles of varying diversities provided superior accuracy to other drift handling approaches. Their approach improved upon previous methods, but required two ensembles before concept drift was detected (when the concept is stable) and four ensembles after concept drift occurred. Computational overheads are associated with maintaining ensembles. Since an additional two ensembles were required after concept drift, the question of how long these two additional ensembles should remain in memory deserves further investigation. This dissertation systematically investigated how decisions regarding maintenance of additional ensembles affect the accuracy of ensemble classifiers. The impact of an MTS parameter on the DDD algorithm was studied in detail using artificial and real world data. Conducting tests with artificial and real world datasets, results showed that in many cases the number of time steps four ensembles are in memory can be reduced without sacrificing prequential accuracy. The use of the MTS parameter with the DDD algorithm gave comparable and sometimes better average prequential accuracy compared to the Standard DDD and DDD with Reset. 
The impact of changes in $W$ related to settings in the MTS parameter was explored. Following Minku and Yao (2012), additional testing was done by forcing false concept drift at specific times with the artificial data and the real world data. This investigation was done by using the best MTS setting found with different values for the $W$ parameter (weight on the old low diversity ensemble) on artificial and real world datasets. The same settings on the $W$ parameter were used with the Standard DDD algorithm for comparison. While increasing $W$ did seem to help produce improved accuracy when false positive concept drift occurred in a few cases, it was clear that the accuracy of Best MTS did not suffer greatly when false positive concept drift occurred, regardless of $W$ setting. 


\section{Appendices}

\section{Appendix A - Comparison of Various MTS Settings - Circle}

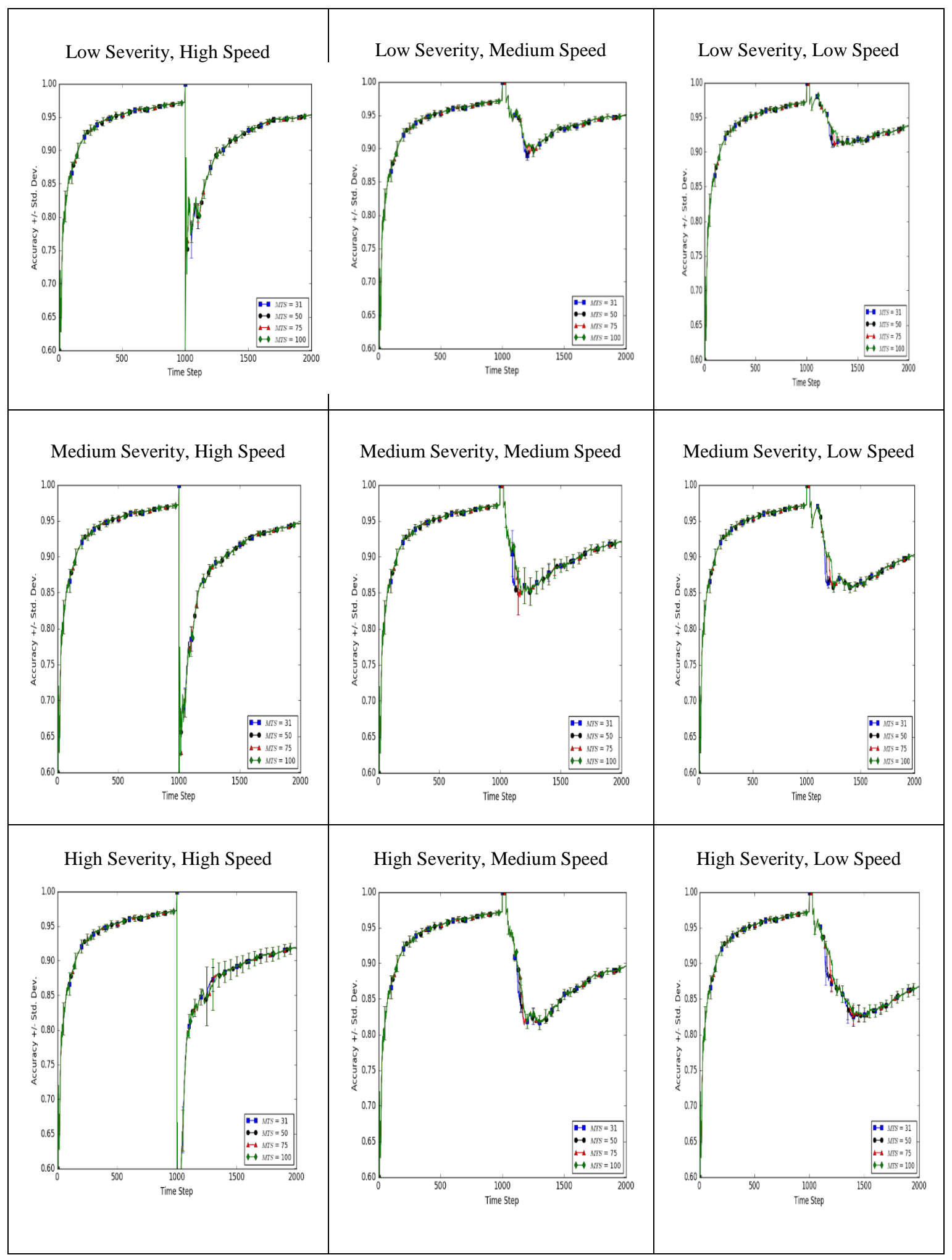




\section{Appendix A - Comparison of Various MTS Settings - SineV}

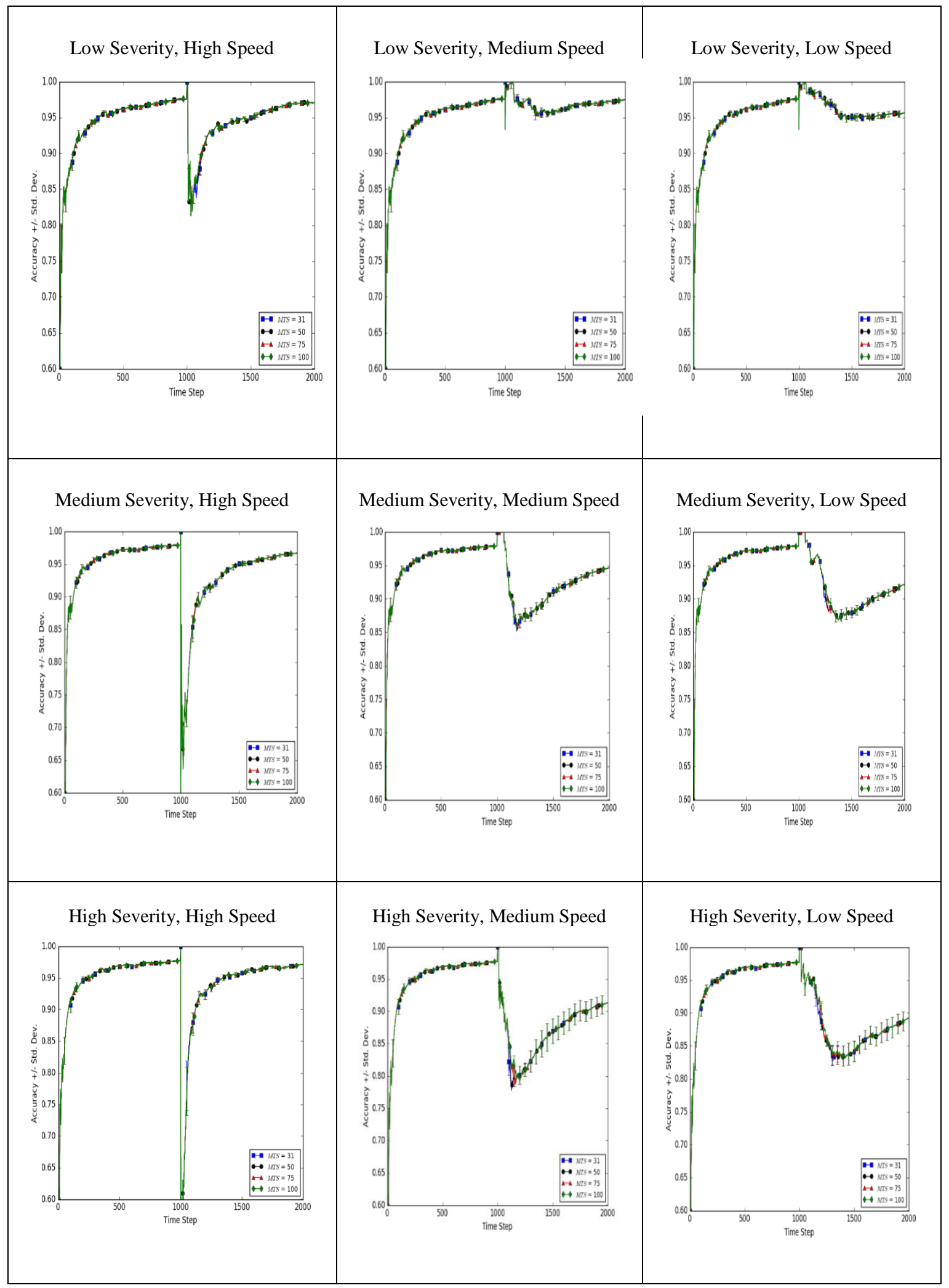




\section{Appendix A - Comparison of Various MTS Settings - SineH}

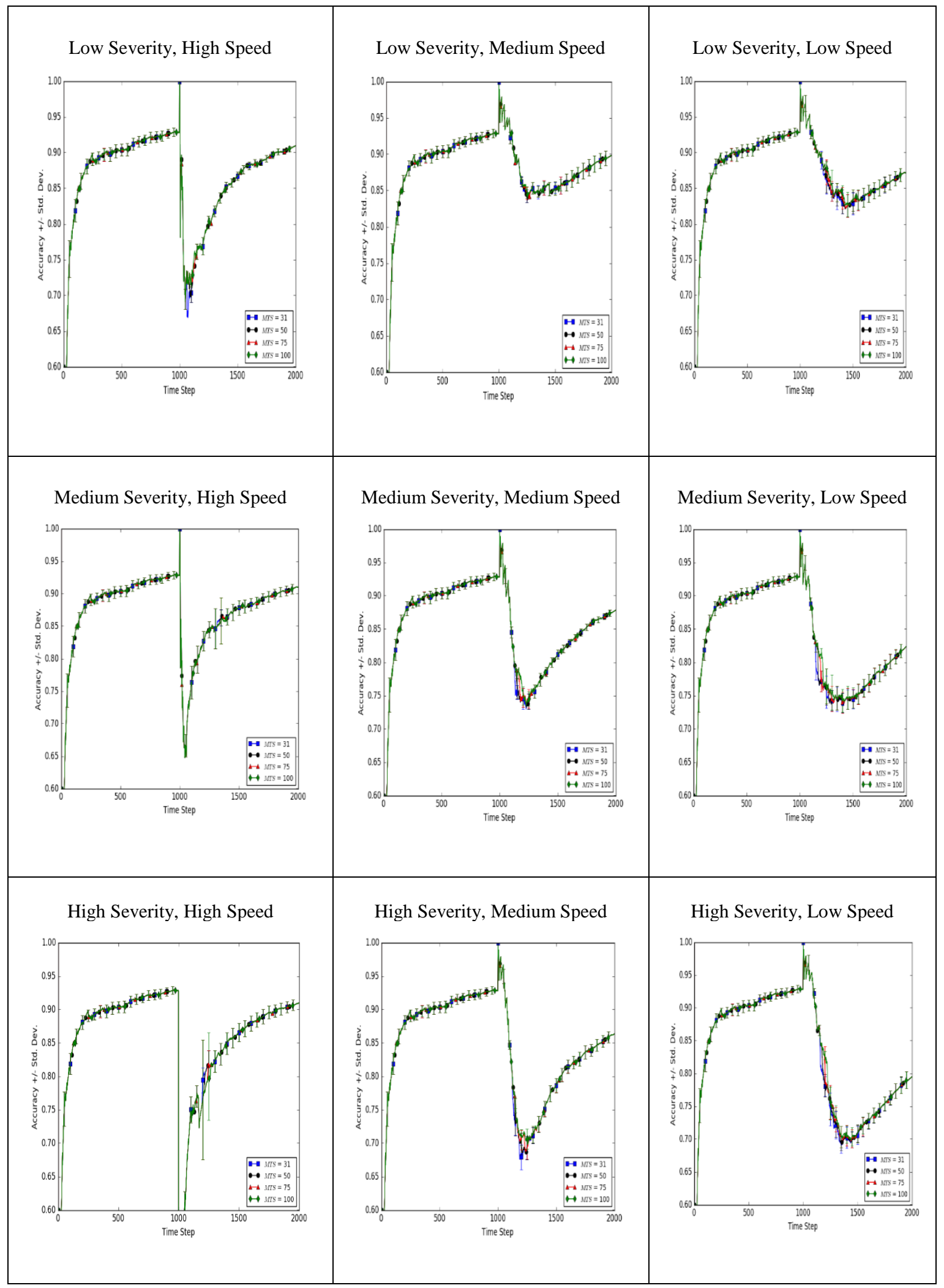




\section{Appendix A - Comparison of Various MTS Settings - Line}

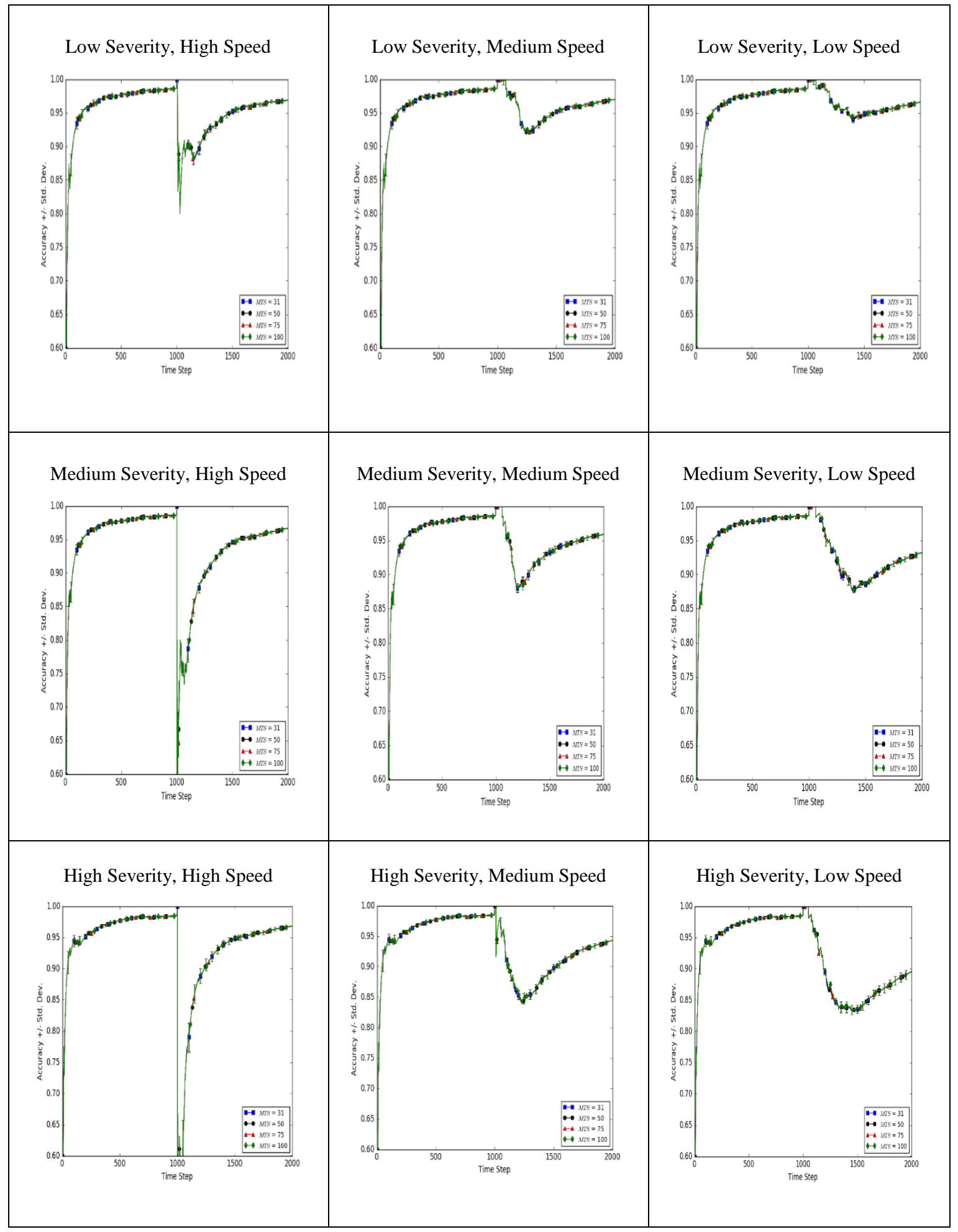




\section{Appendix A - Comparison of Various MTS Settings - Plane}

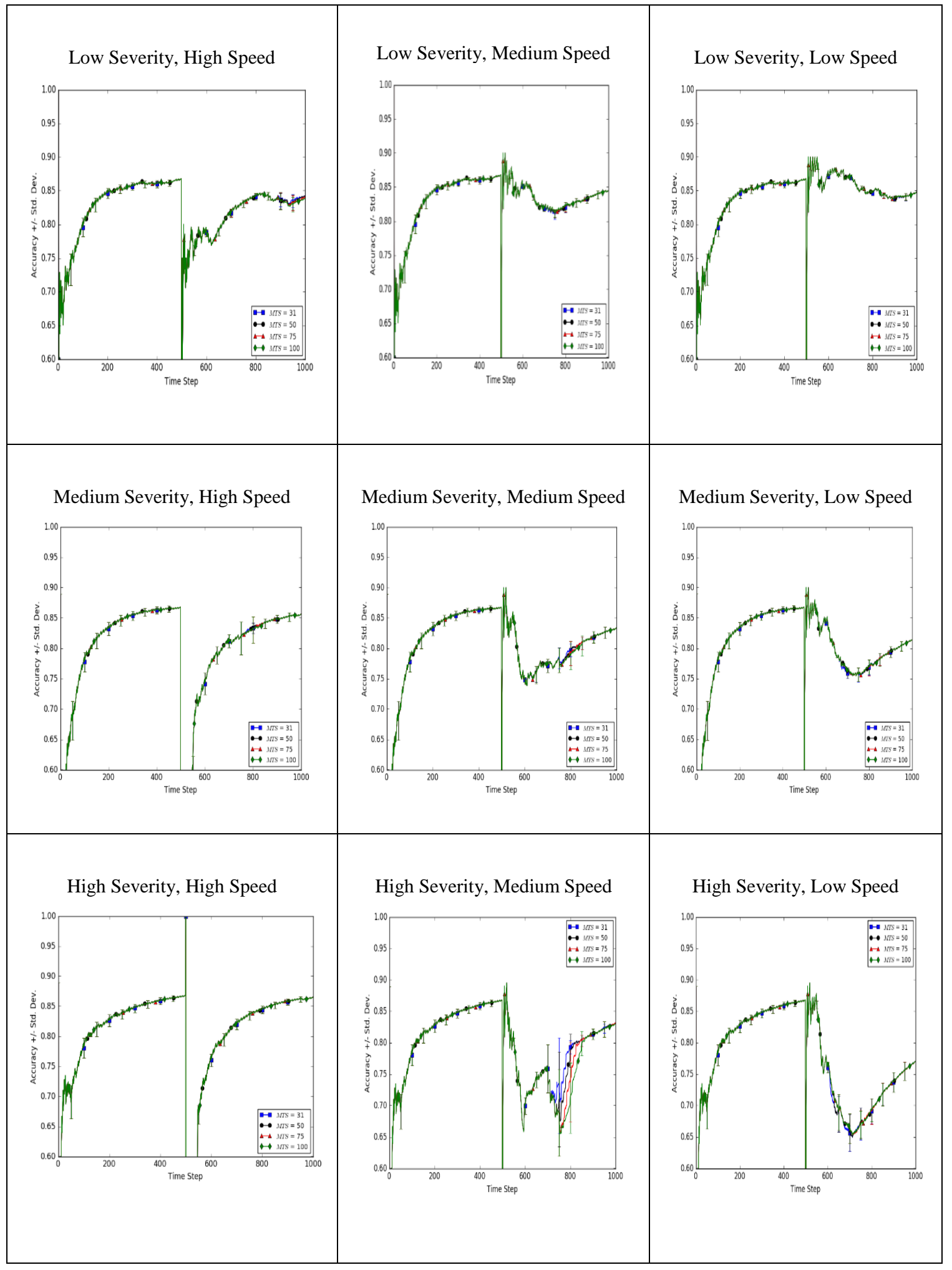




\section{Appendix A - Comparison of Various MTS Settings - Boolean}

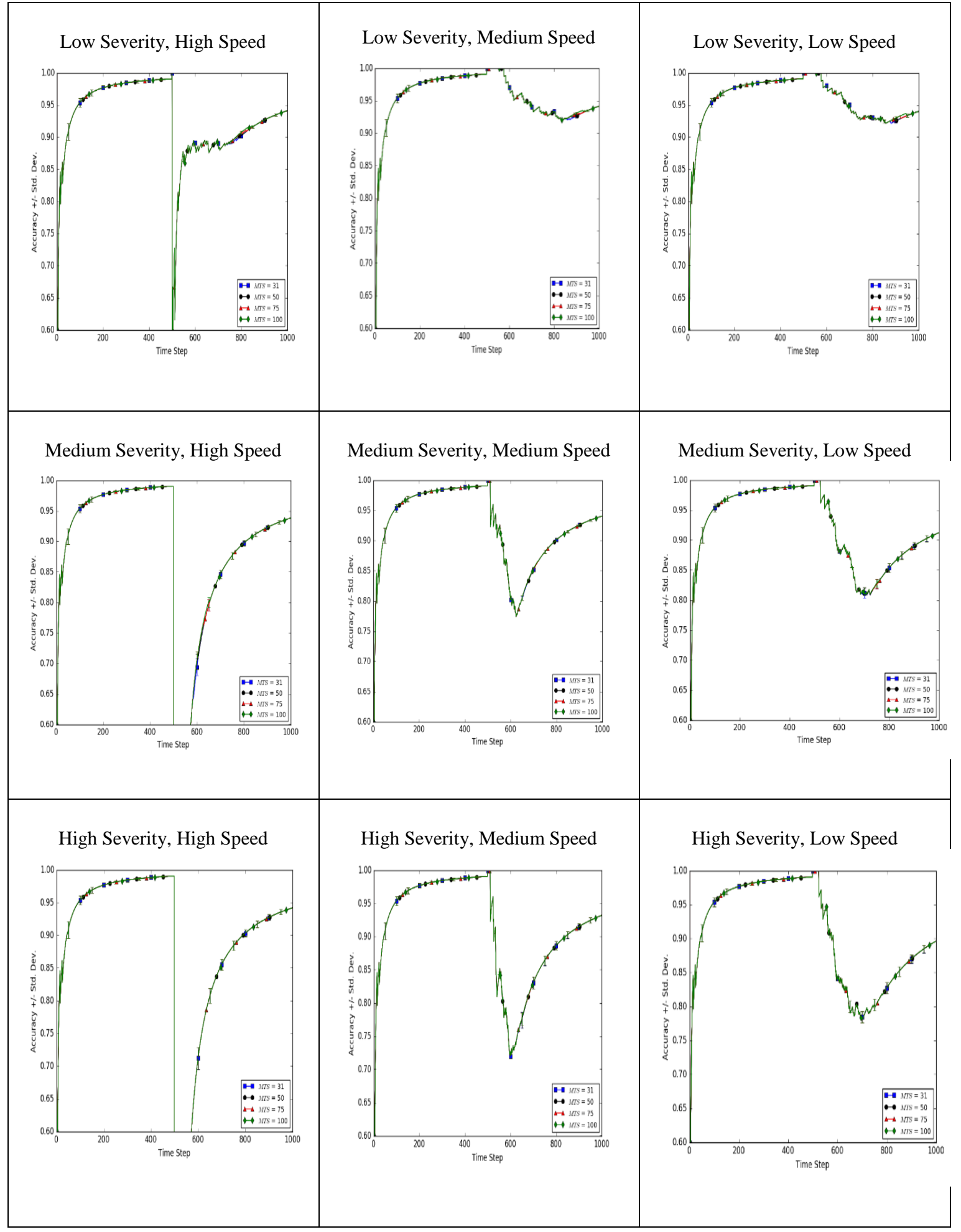


Appendix A - Comparison of Various MTS Settings - Electricity

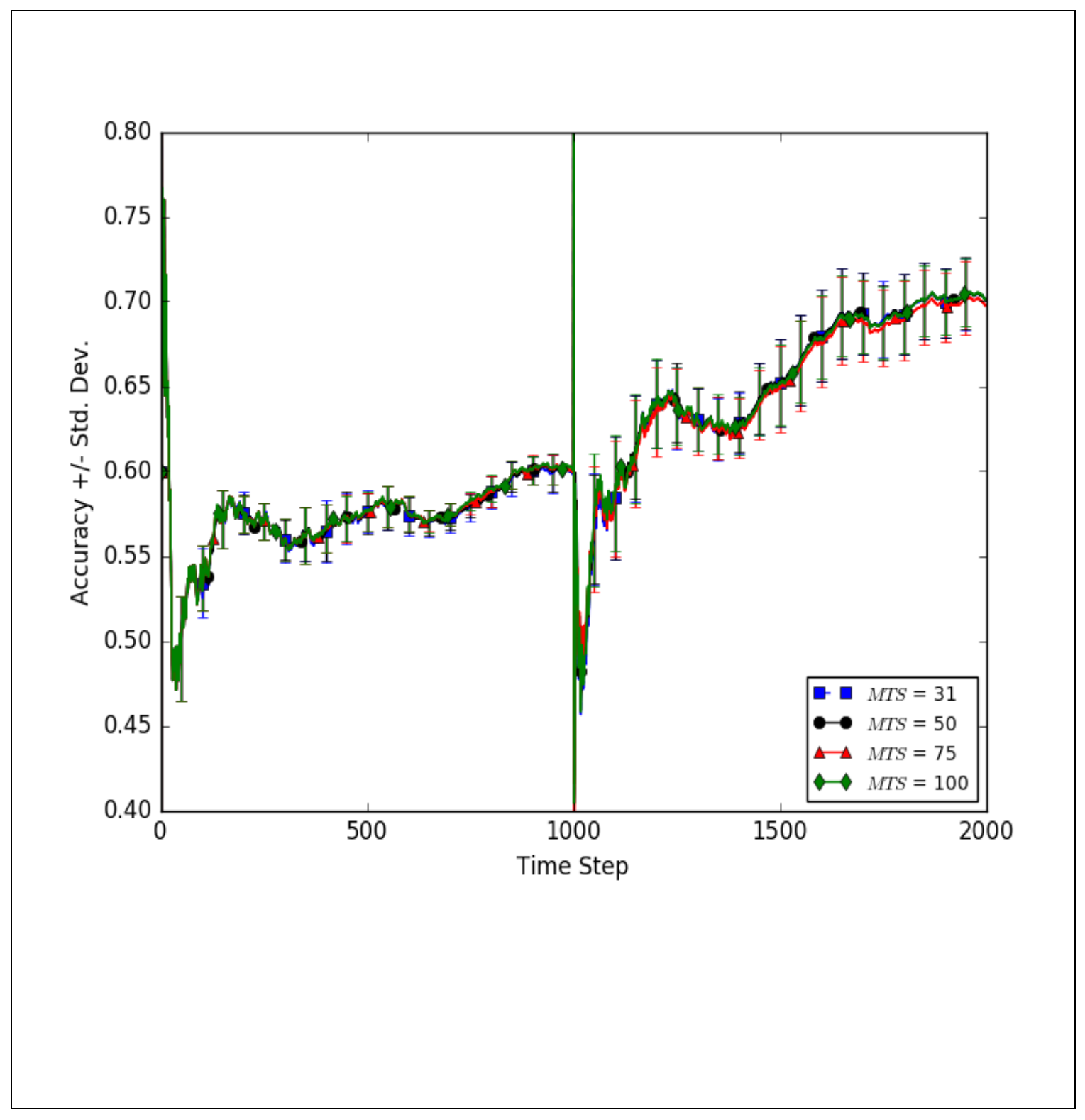


Appendix A - Comparison of Various MTS Settings - Forest CoverType

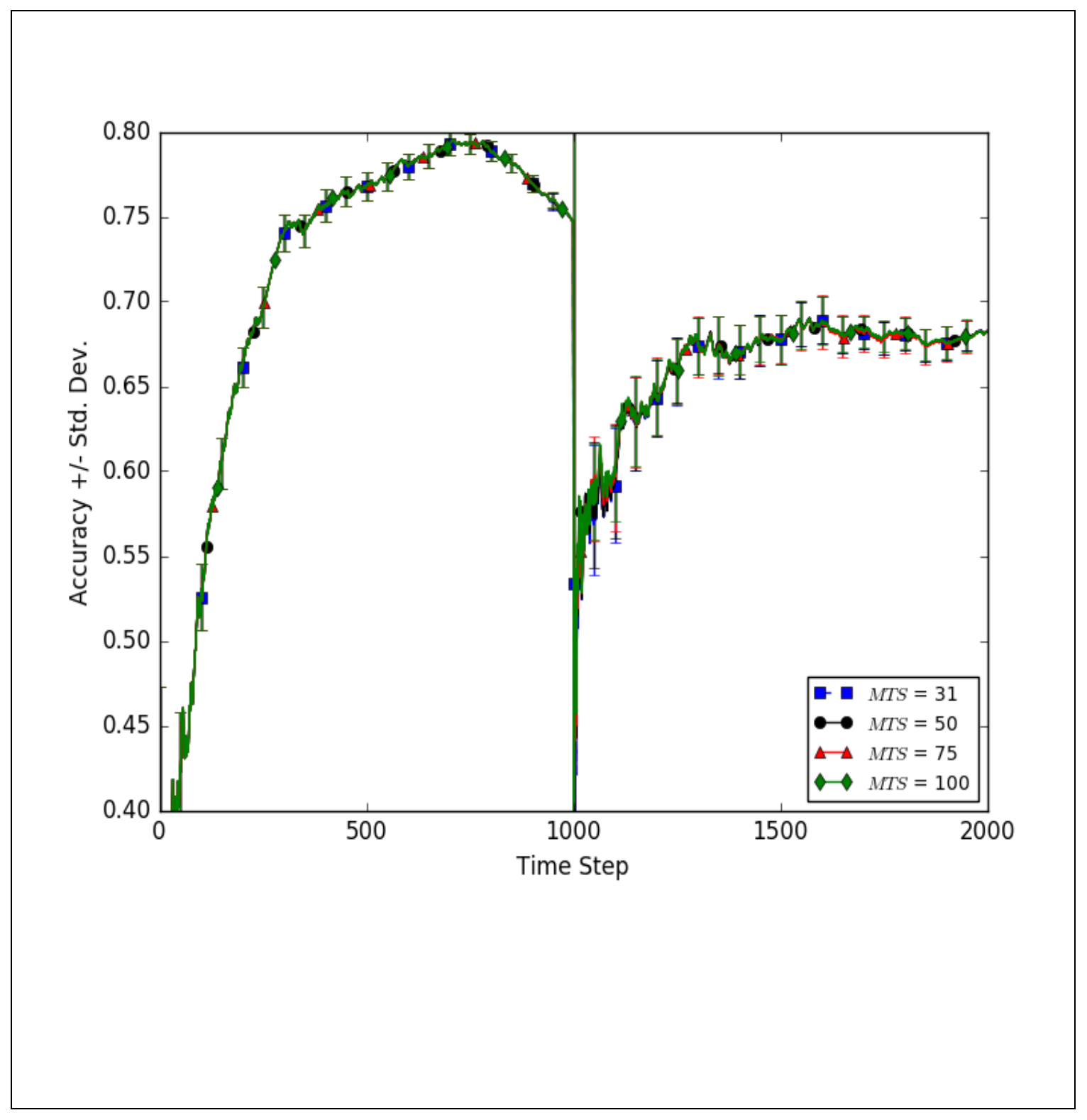


Appendix A - Comparison of Various MTS Settings - KDD 1999

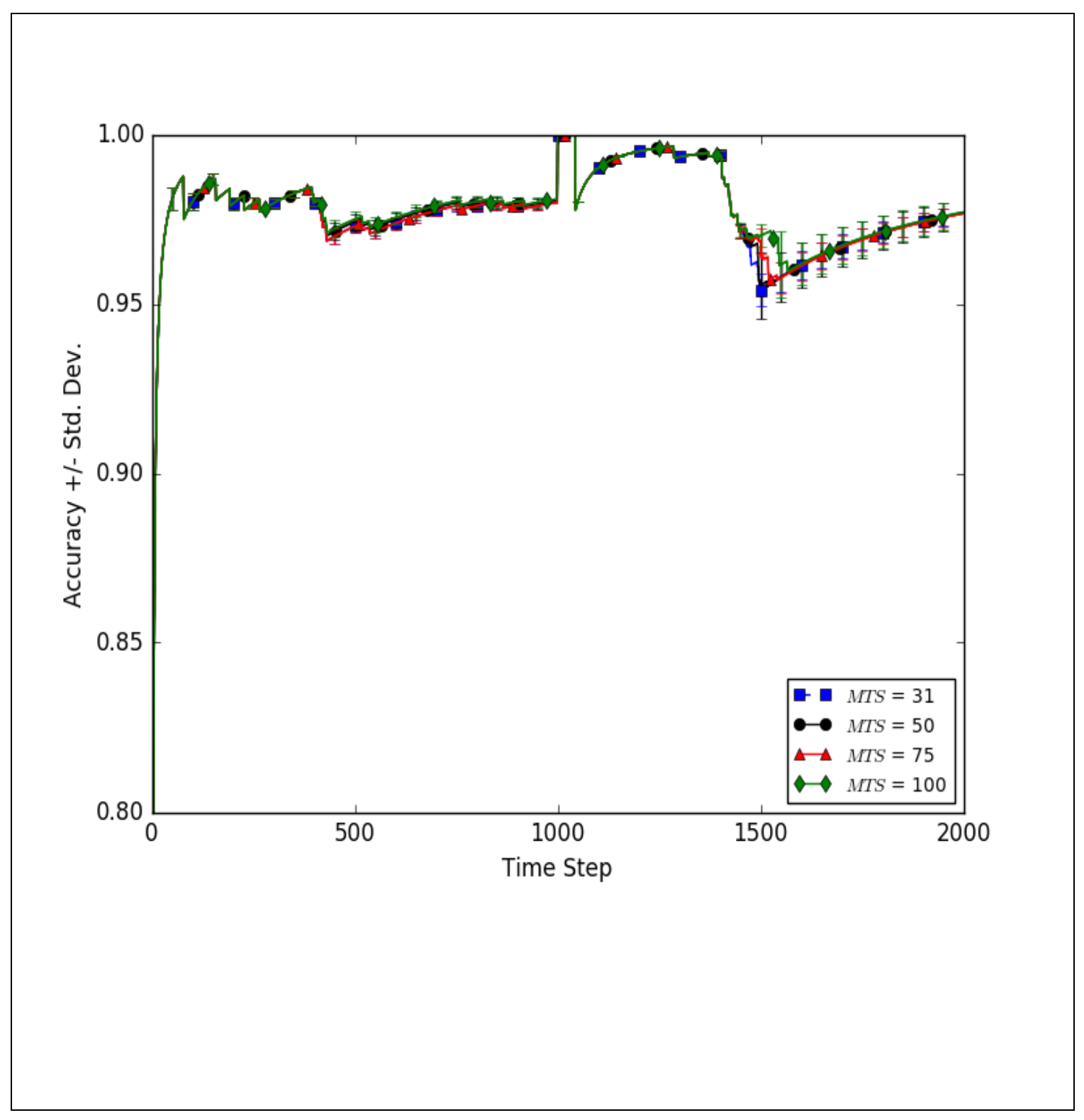


Appendix B - Frequency of MTS Values - Artificial Data

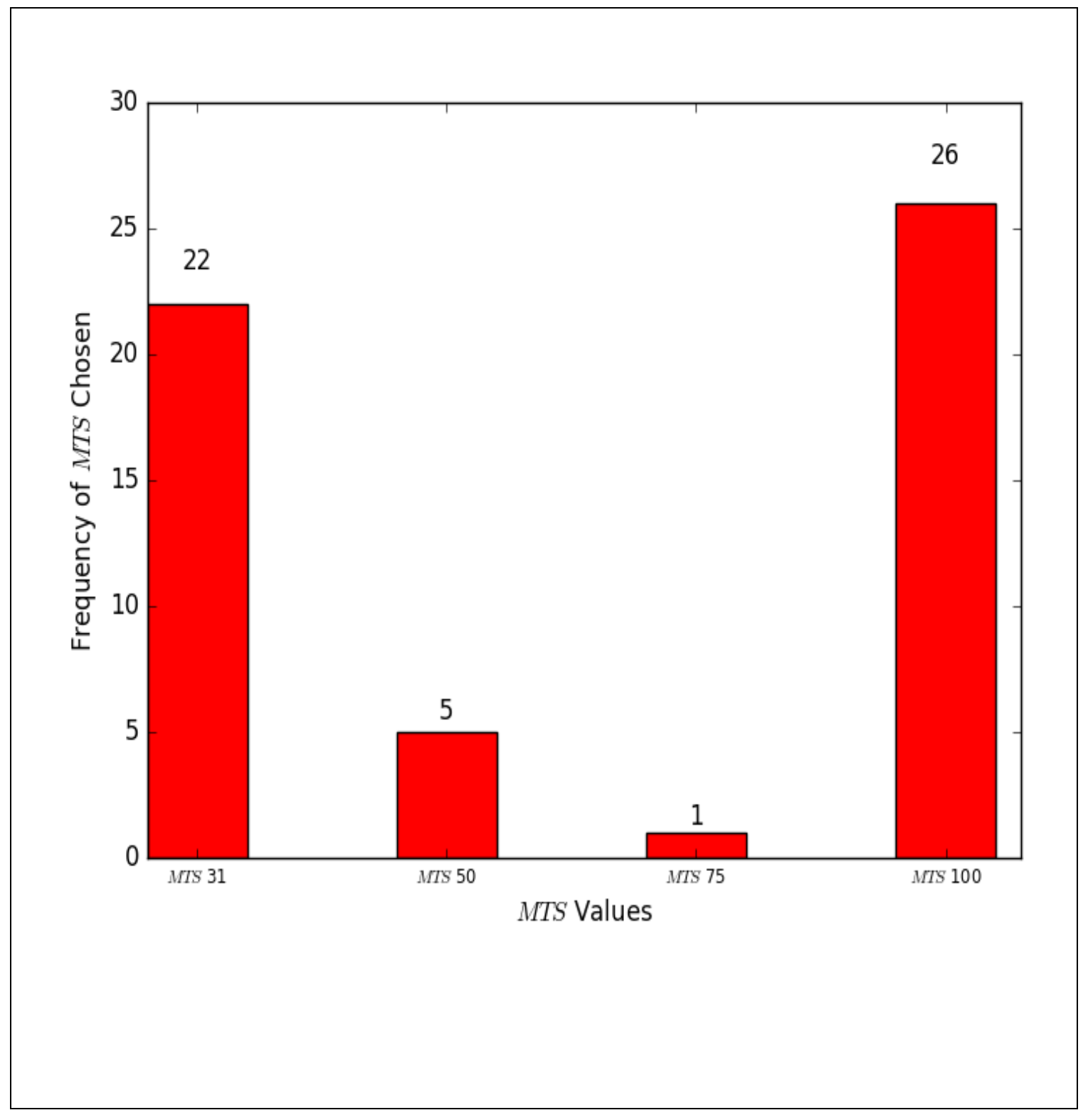


Appendix B - Frequency of MTS Values - Real World Data

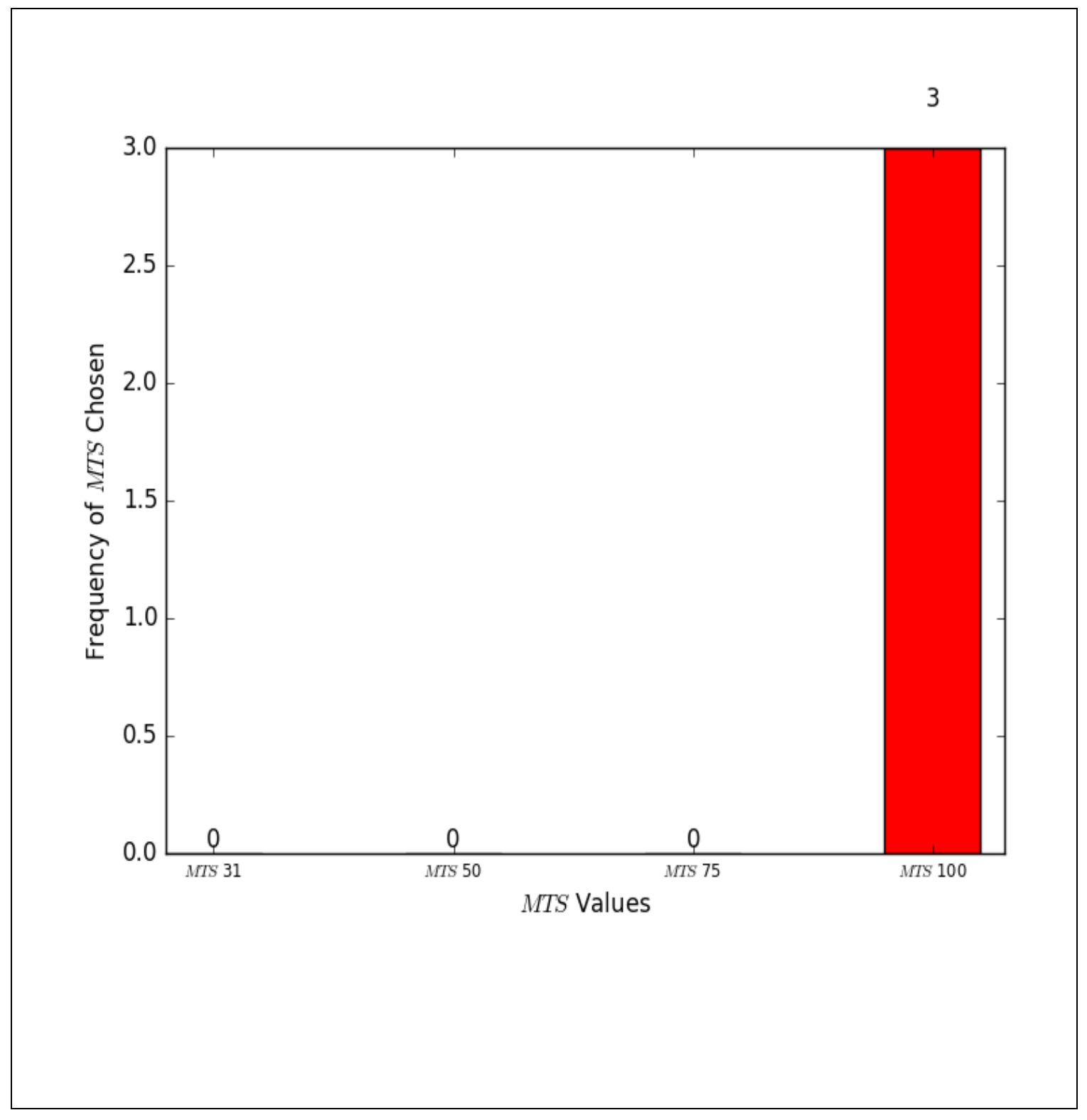




\section{Appendix C - Comparison of Best MTS, DDD, and DDD with Reset - Circle}

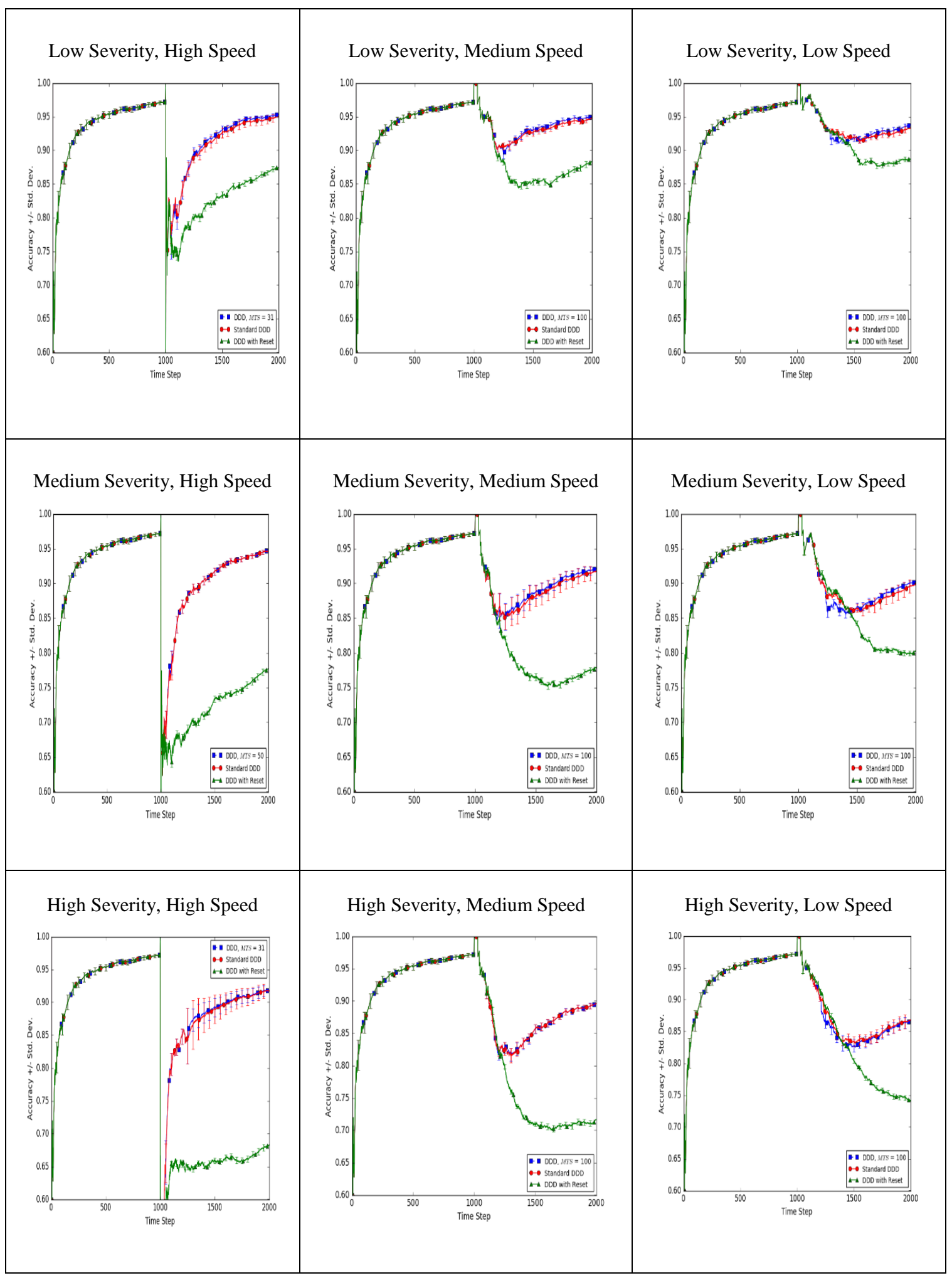




\section{Appendix C - Comparison of Best MTS, DDD, and DDD with Reset - SineV}

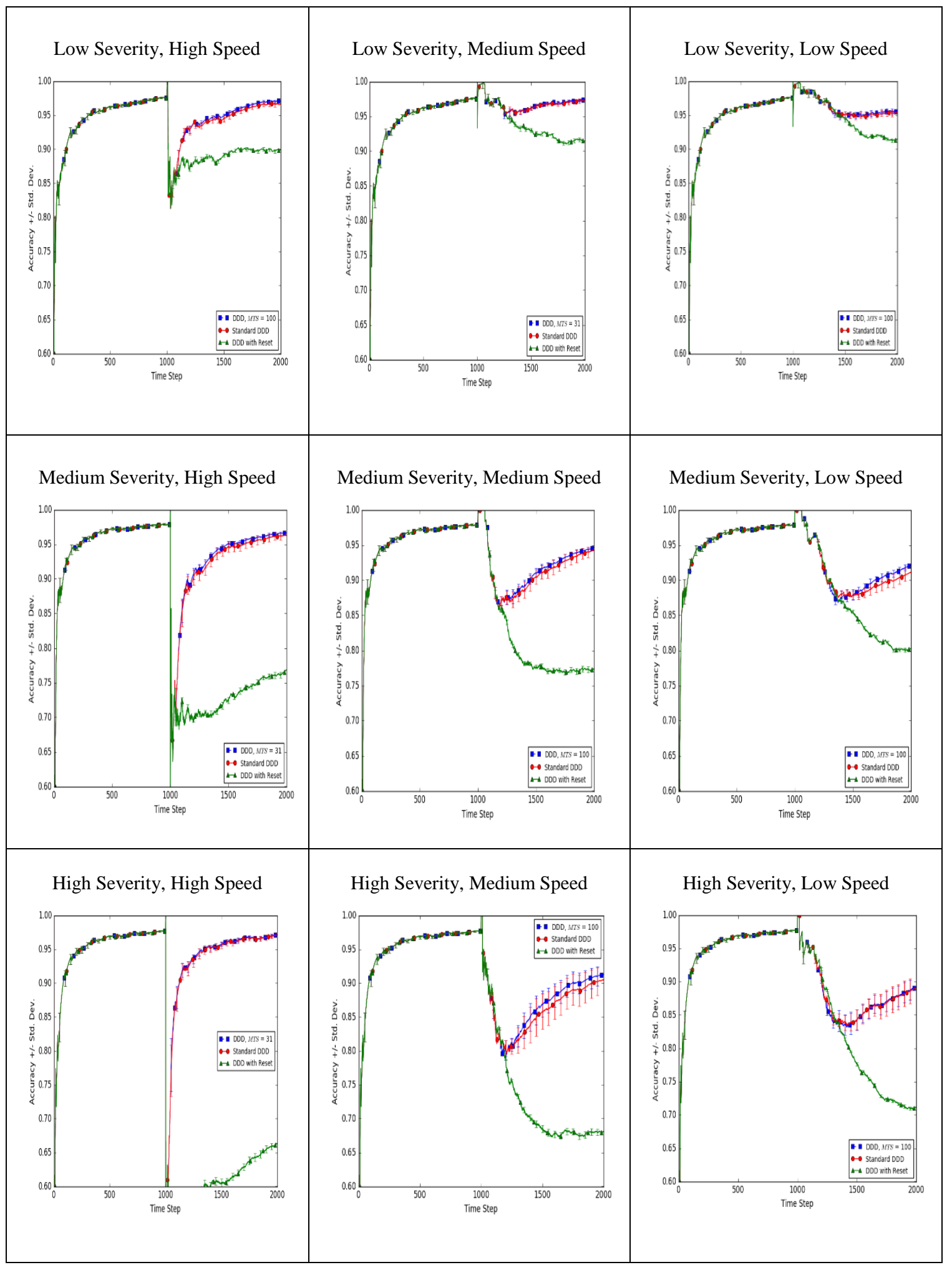




\section{Appendix C - Comparison of Best MTS, DDD, and DDD with Reset - SineH}

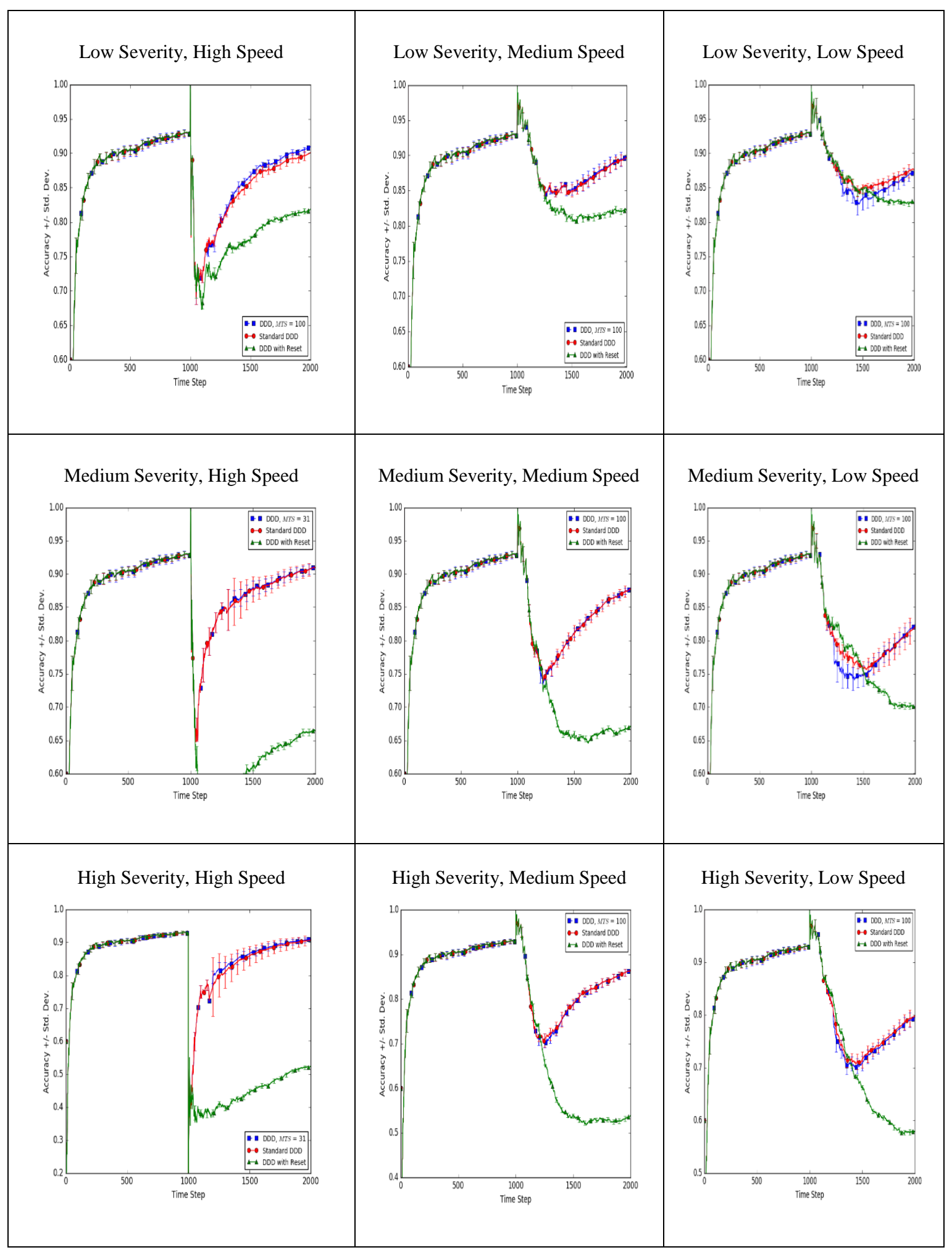




\section{Appendix C - Comparison of Best MTS, DDD, and DDD with Reset - Line}

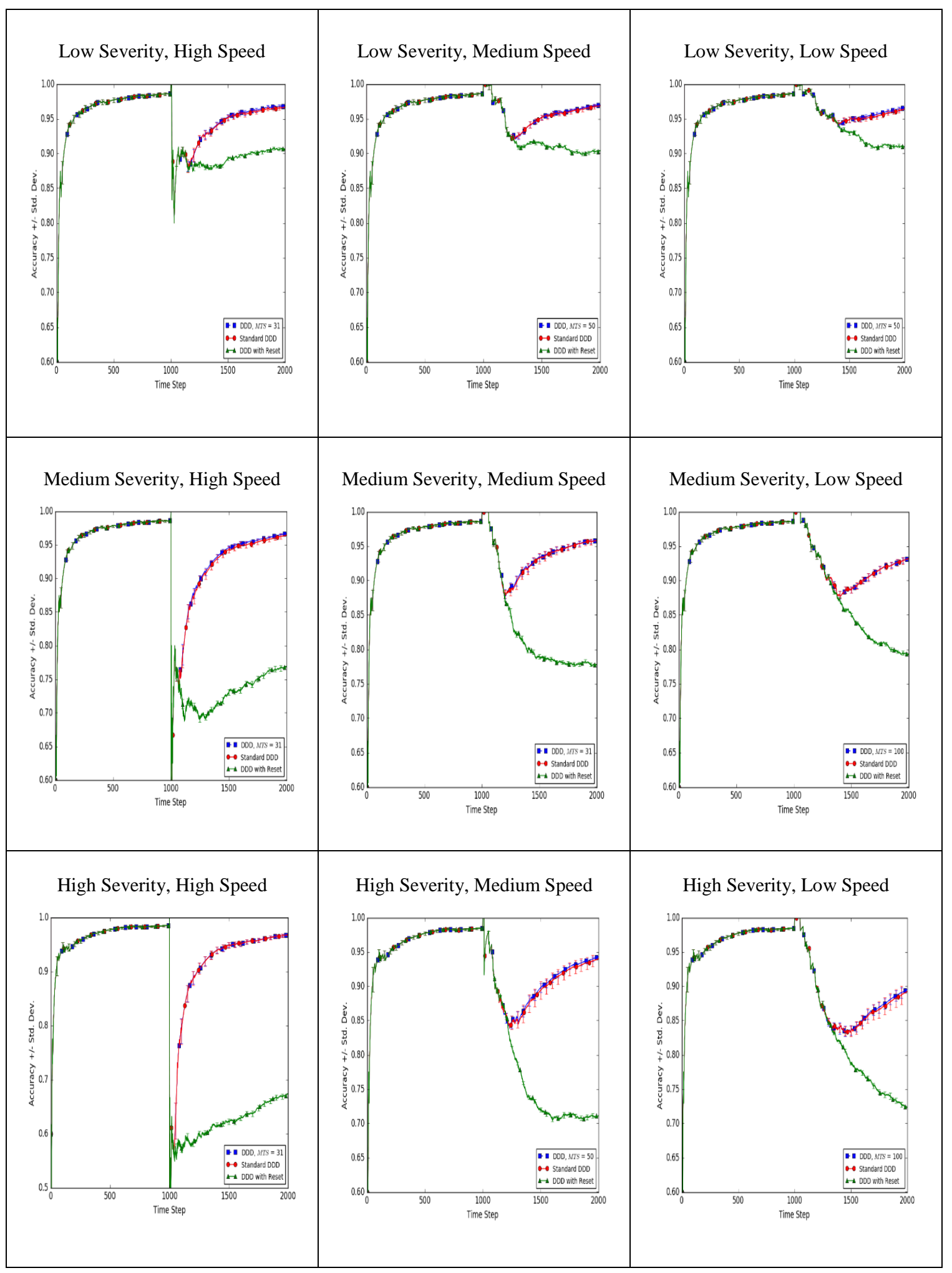




\section{Appendix C - Comparison of Best MTS, DDD, and DDD with Reset - Plane}

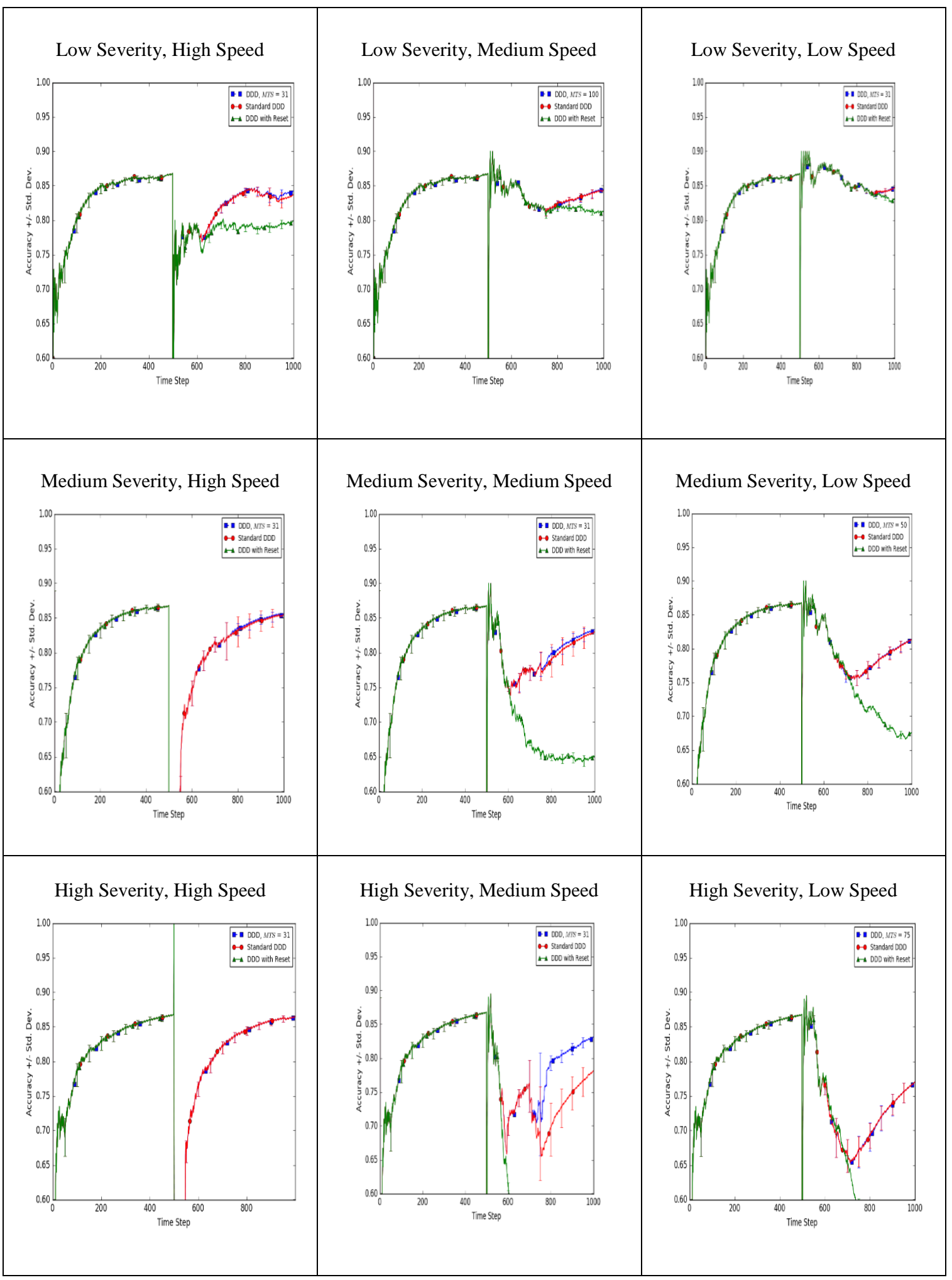




\section{Appendix C - Comparison of Best MTS, DDD, and DDD with Reset - Boolean}

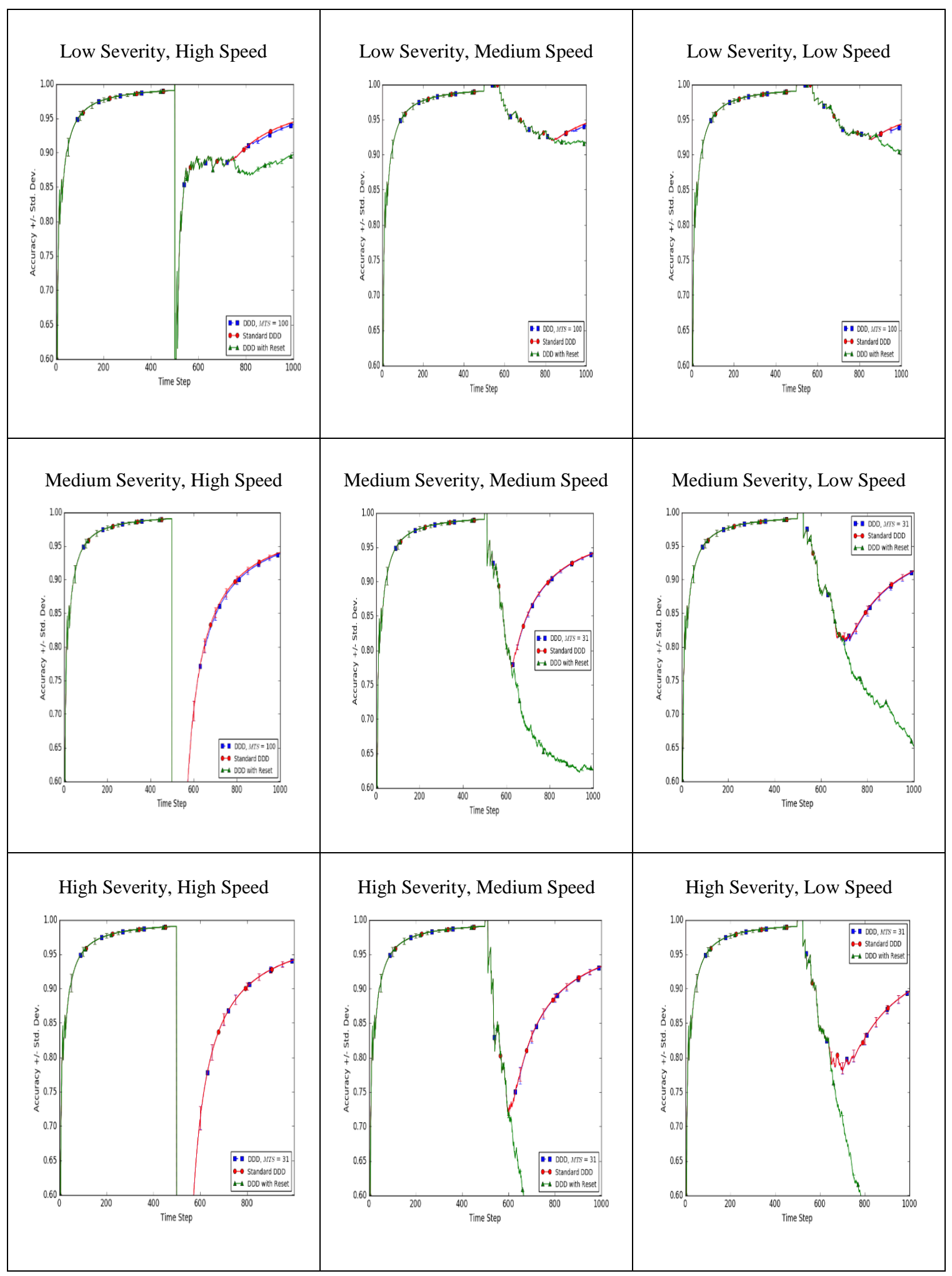


Appendix C - Comparison of Best MTS, DDD, and DDD with Reset - Electricity

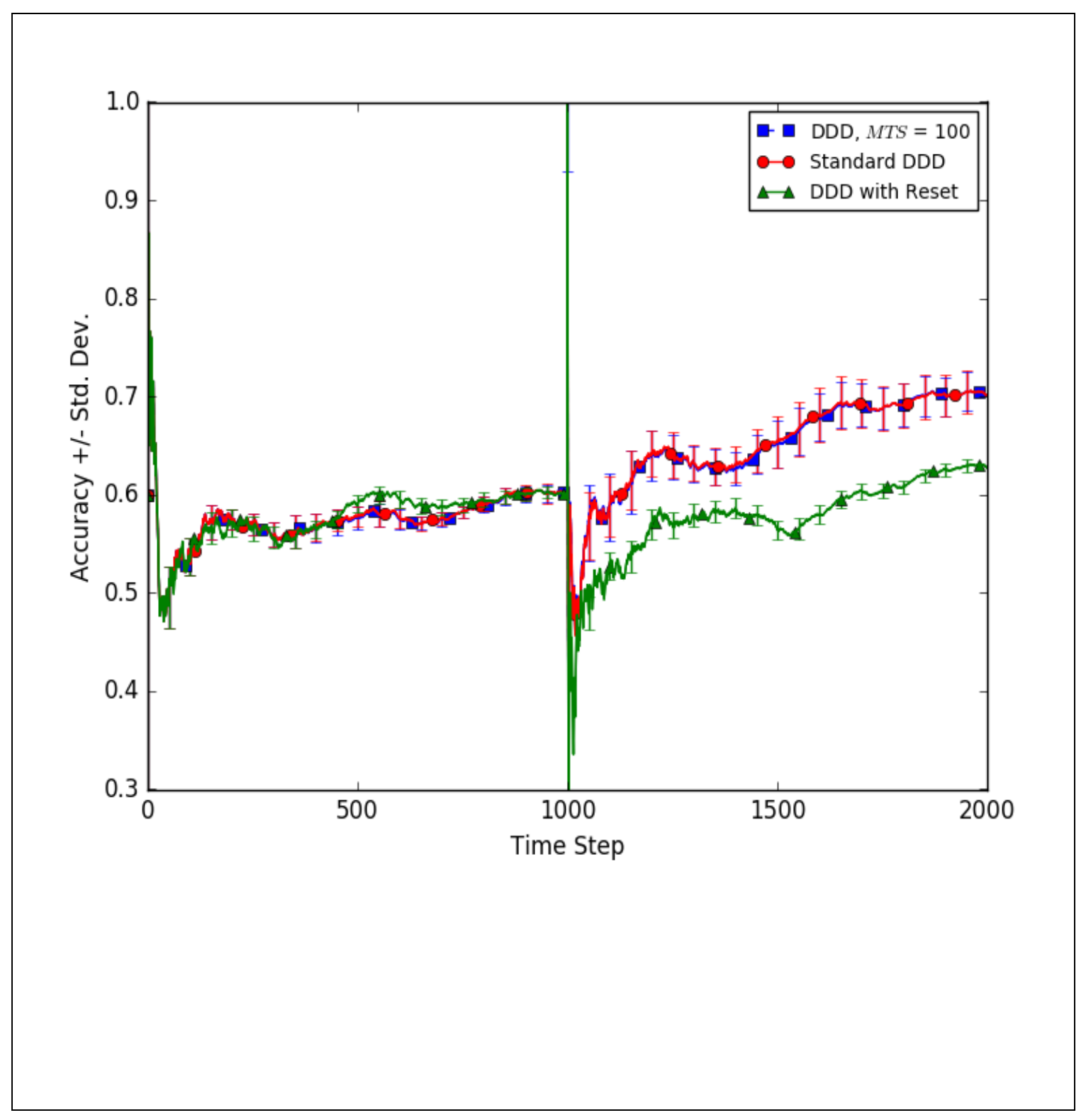


Appendix C - Comparison of Best MTS, DDD, and DDD with Reset - Forest CoverType

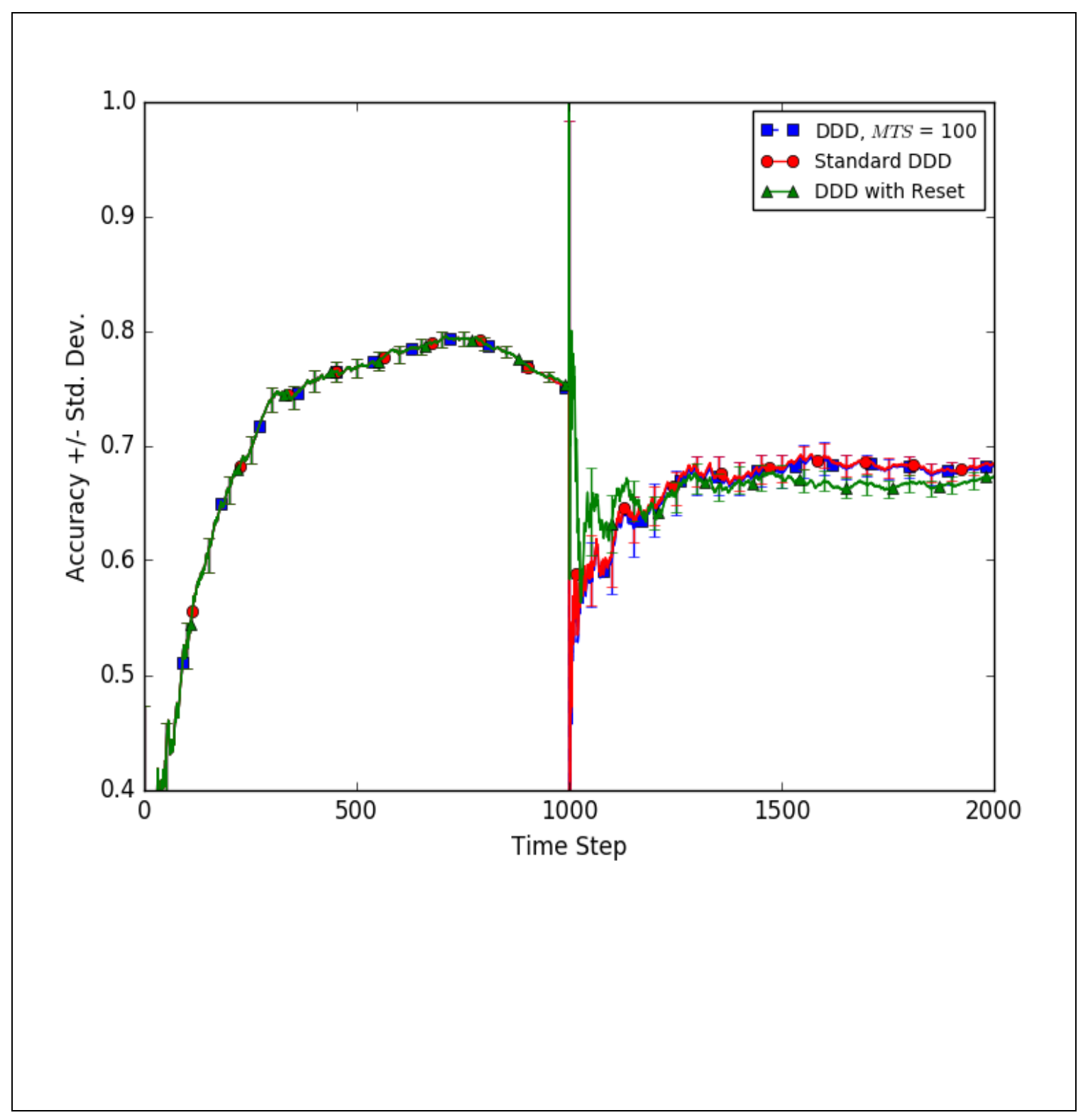


Appendix C - Comparison of Best MTS, DDD, and DDD with Reset - KDD 1999

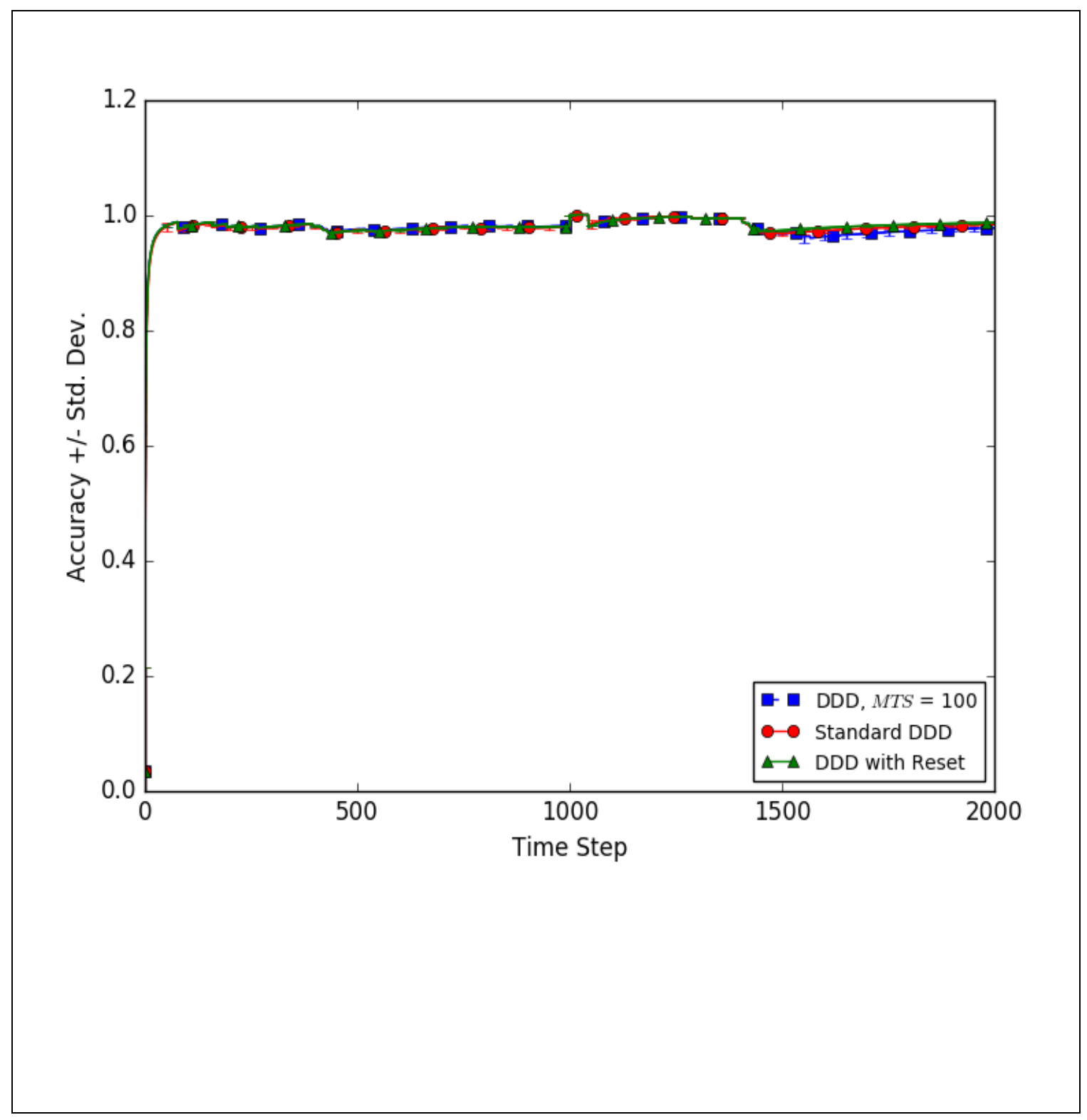


Appendix D - Best MTS and Standard DDD, Average Time Steps in Four Ensembles Circle

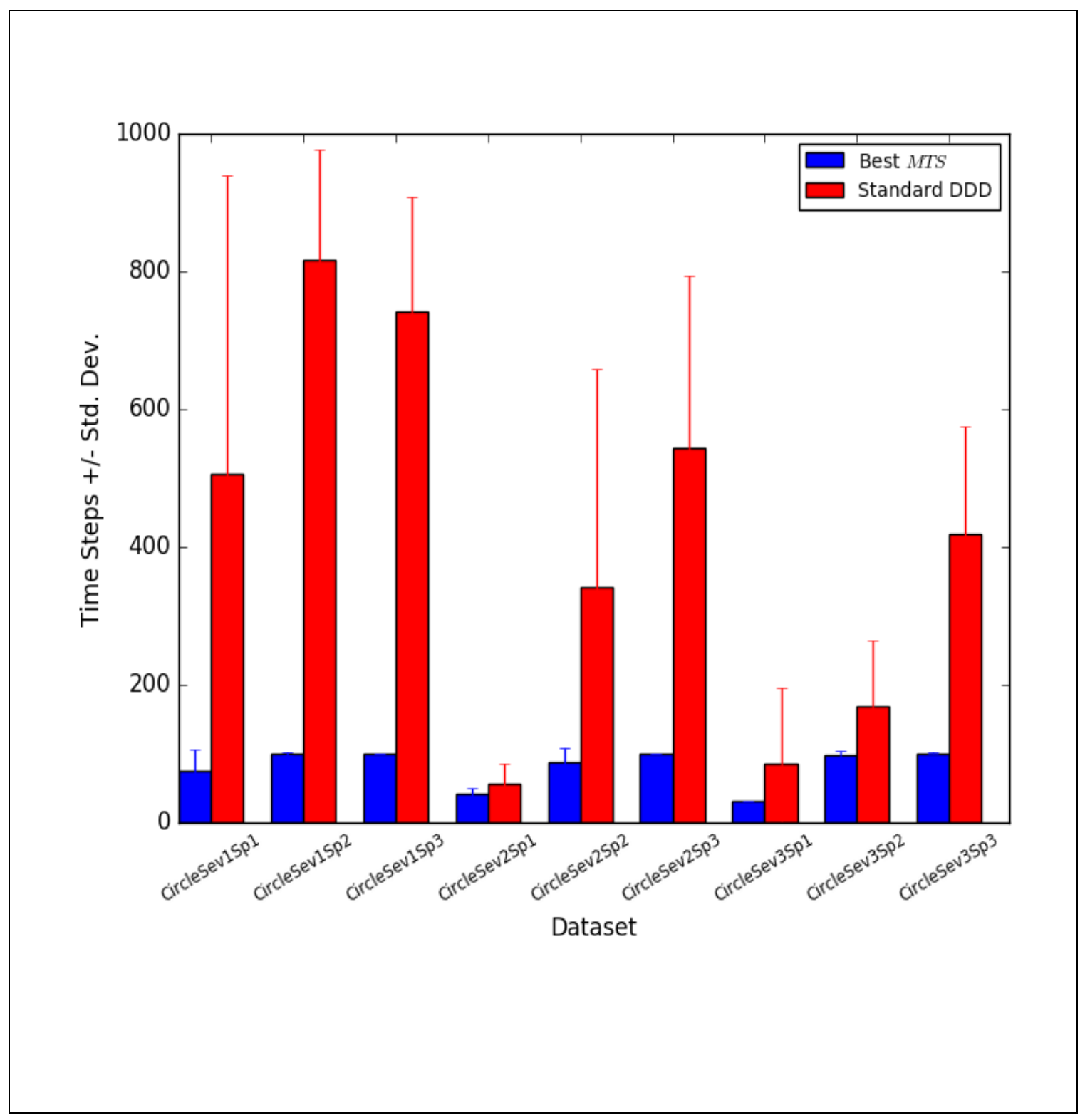


Appendix D - Best MTS and Standard DDD, Average Time Steps in Four Ensembles SineV

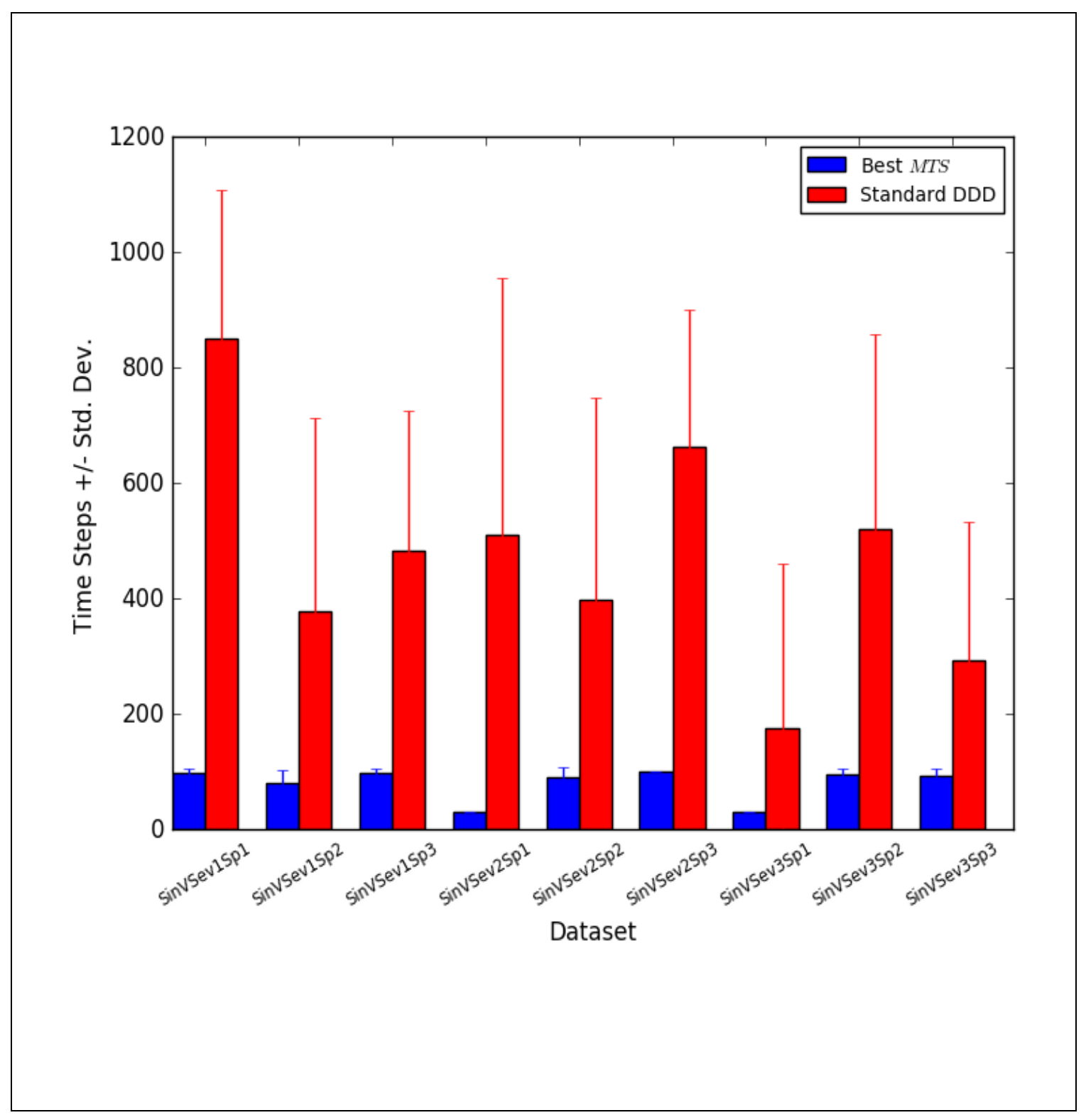


Appendix D - Best MTS and Standard DDD, Average Time Steps in Four Ensembles SineH

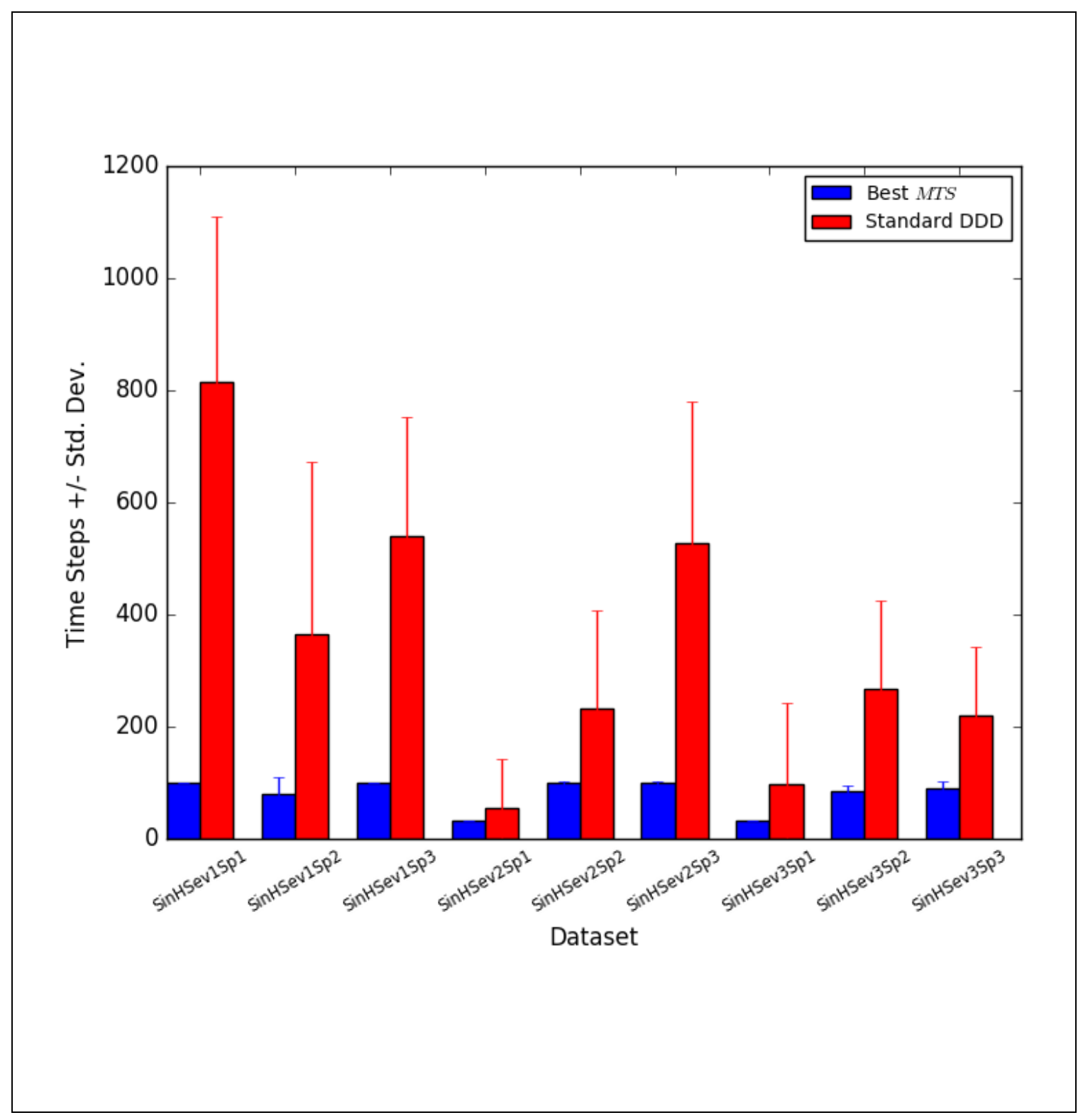


Appendix D - Best MTS and Standard DDD, Average Time Steps in Four Ensembles Line

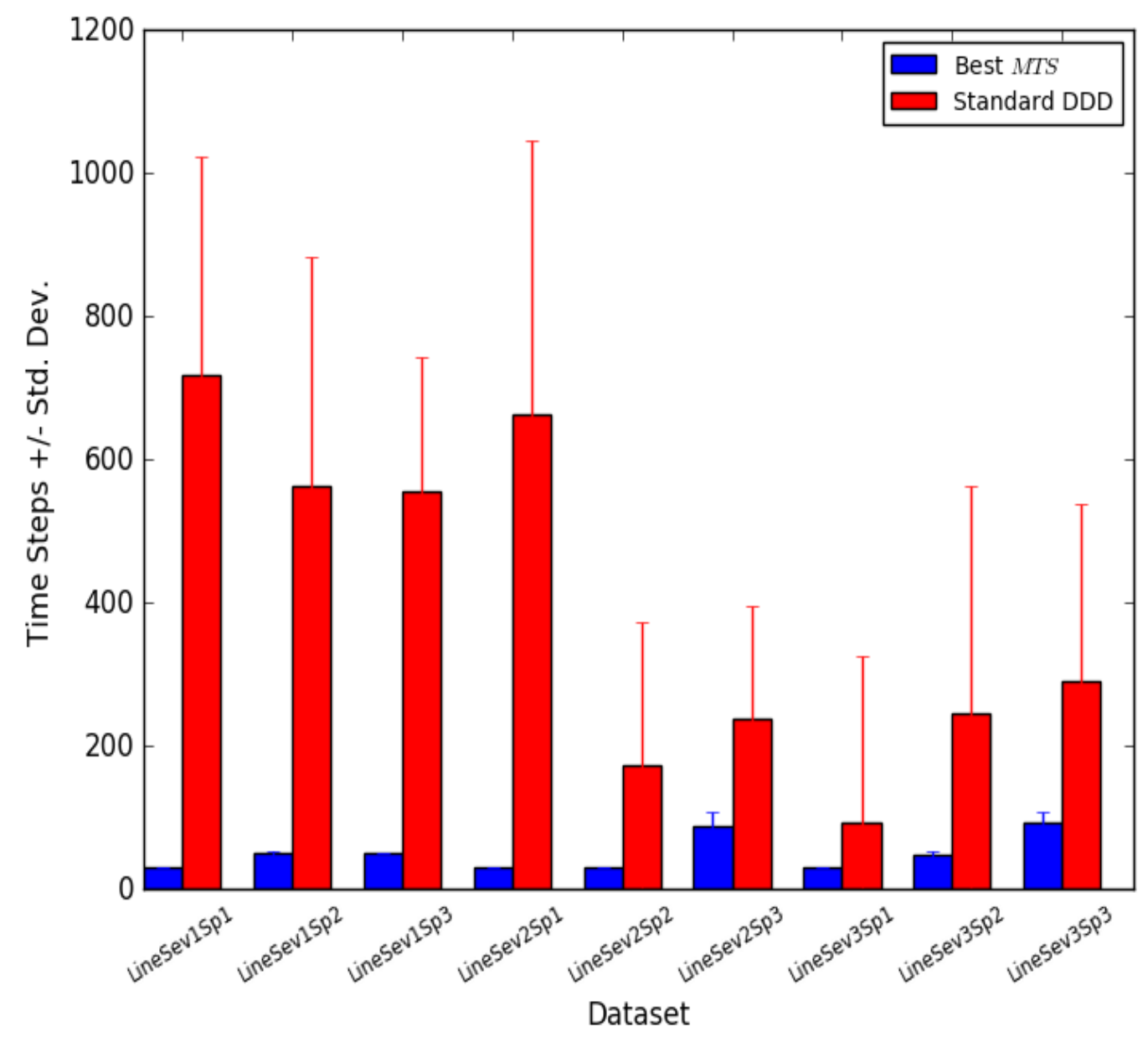


Appendix D - Best MTS and Standard DDD, Average Time Steps in Four Ensembles Plane

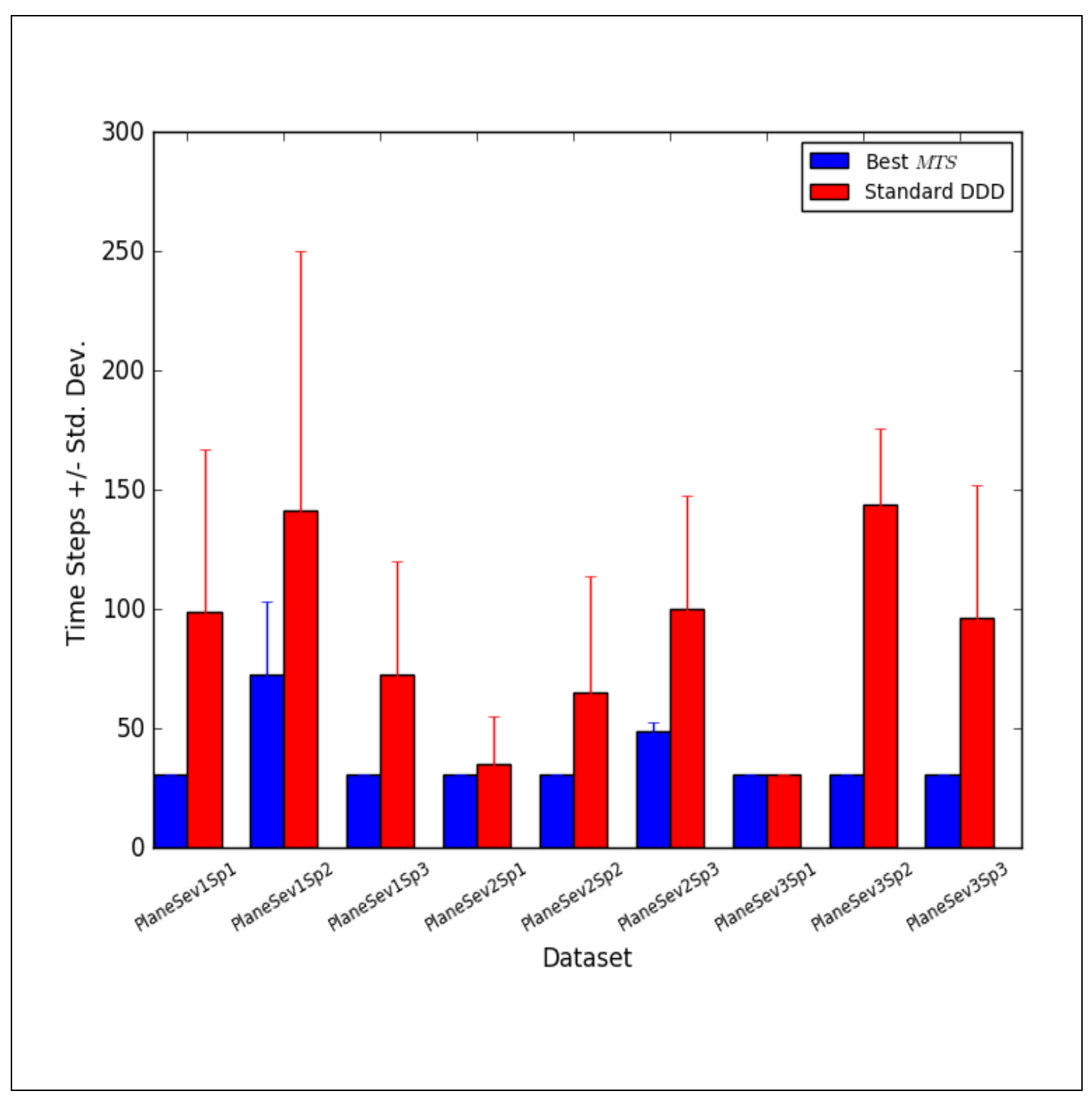


Appendix D - Best MTS and Standard DDD, Average Time Steps in Four Ensembles Boolean

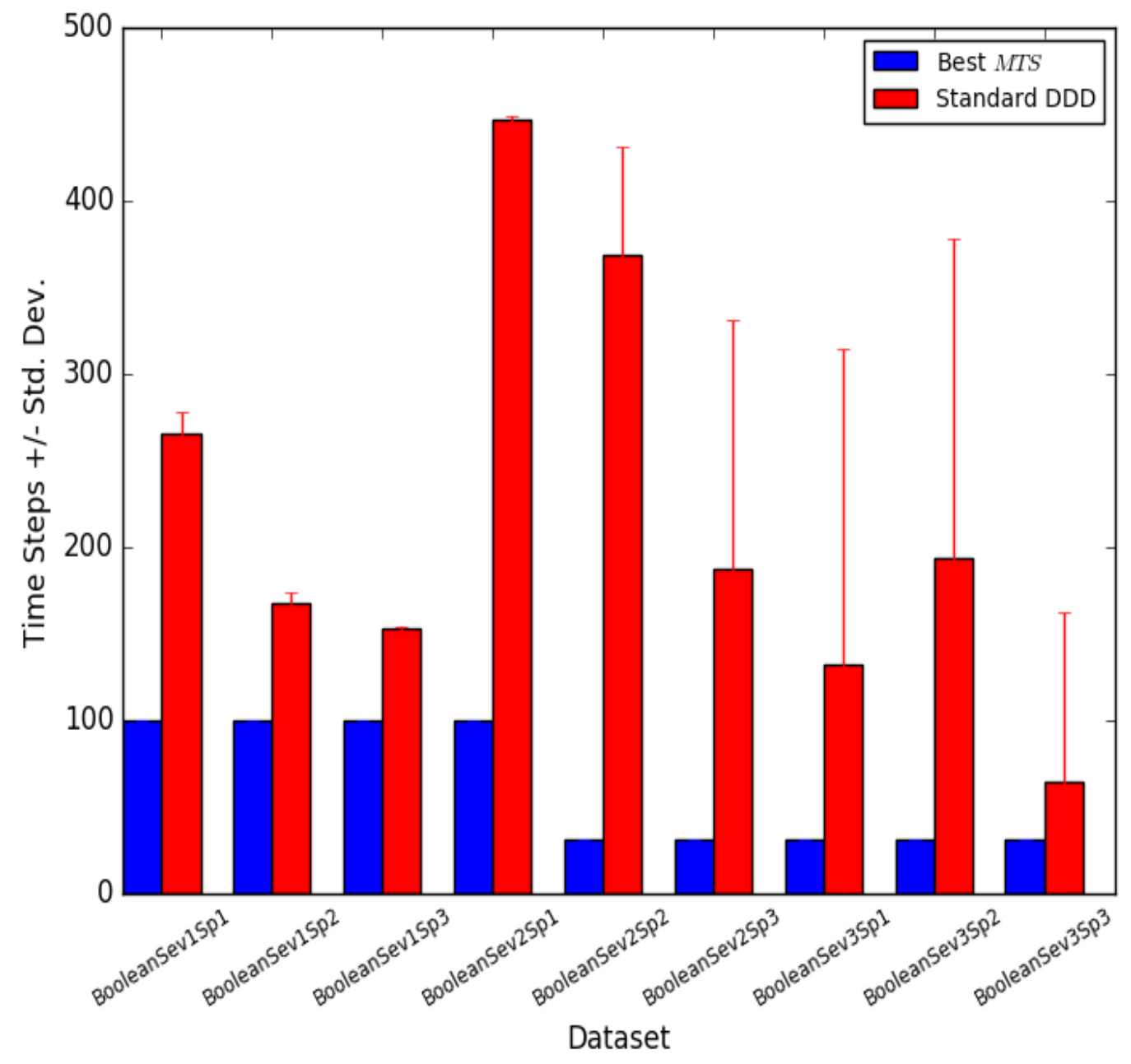


Appendix D - Best MTS and Standard DDD, Average Time Steps in Four Ensembles Real World Data

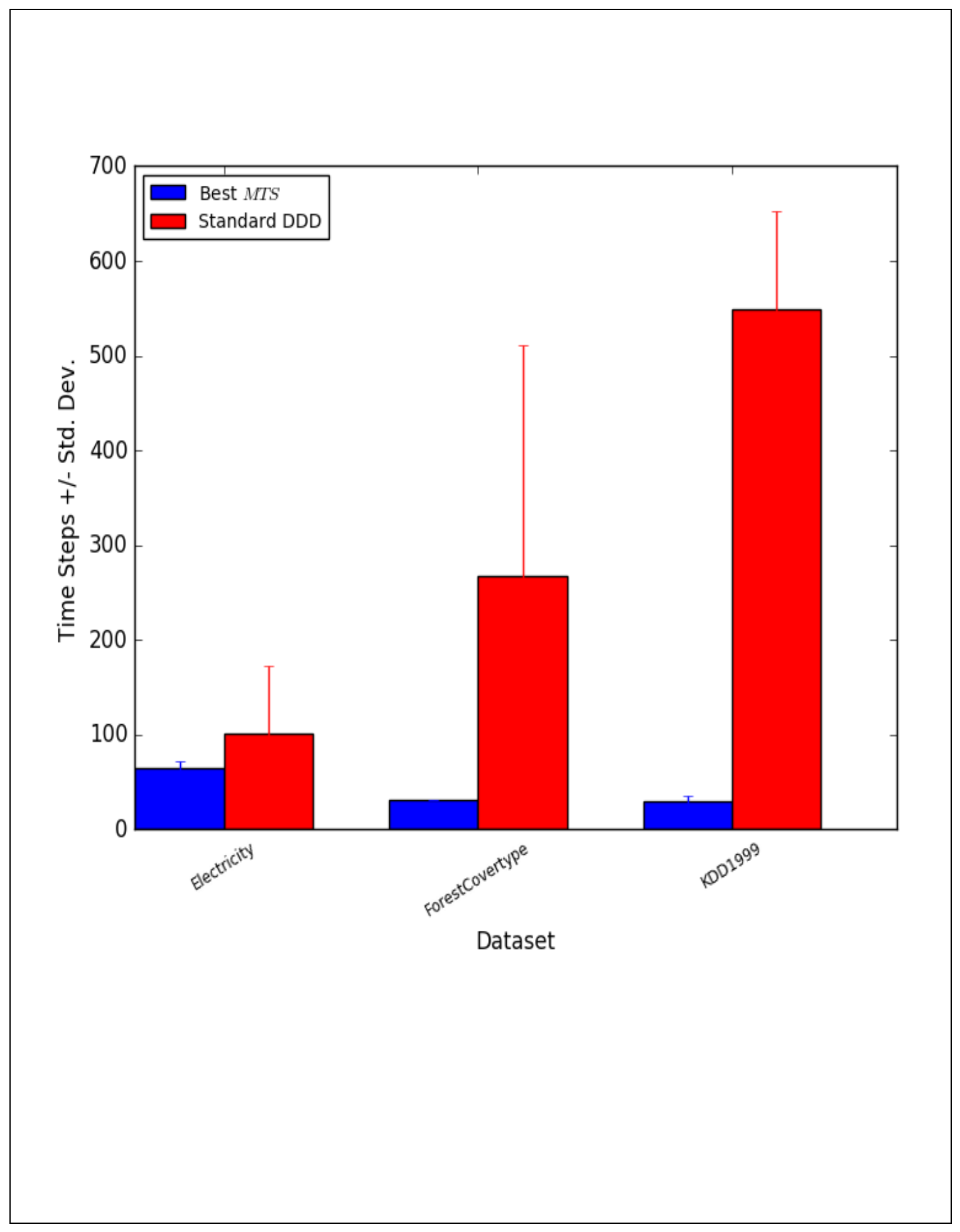




\section{Appendix E - Comparison of Various W Settings using Best MTS - Circle}

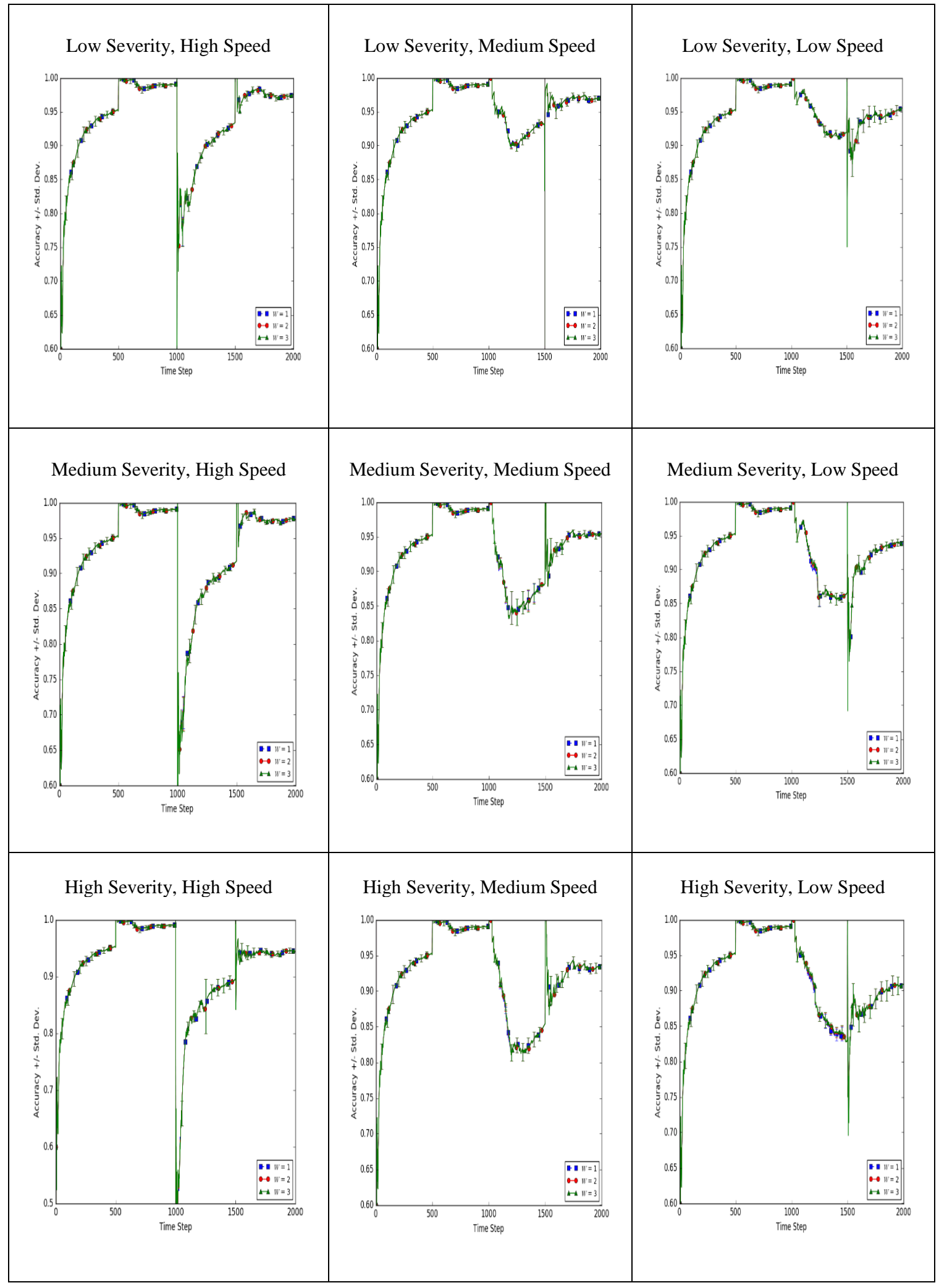




\section{Appendix E - Comparison of Various W Settings using Best MTS - SineV}

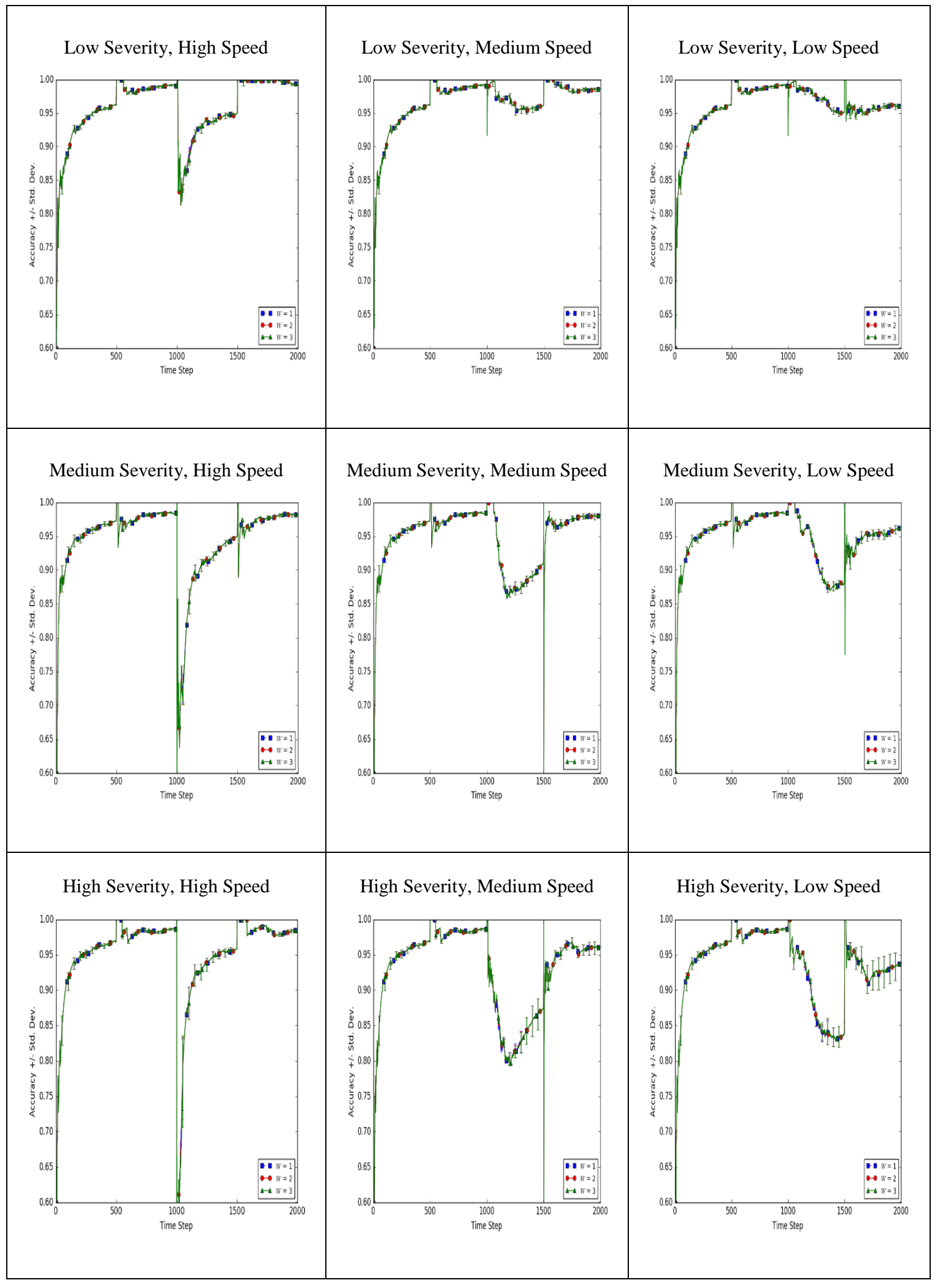




\section{Appendix E - Comparison of Various W Settings using Best MTS - SineH}

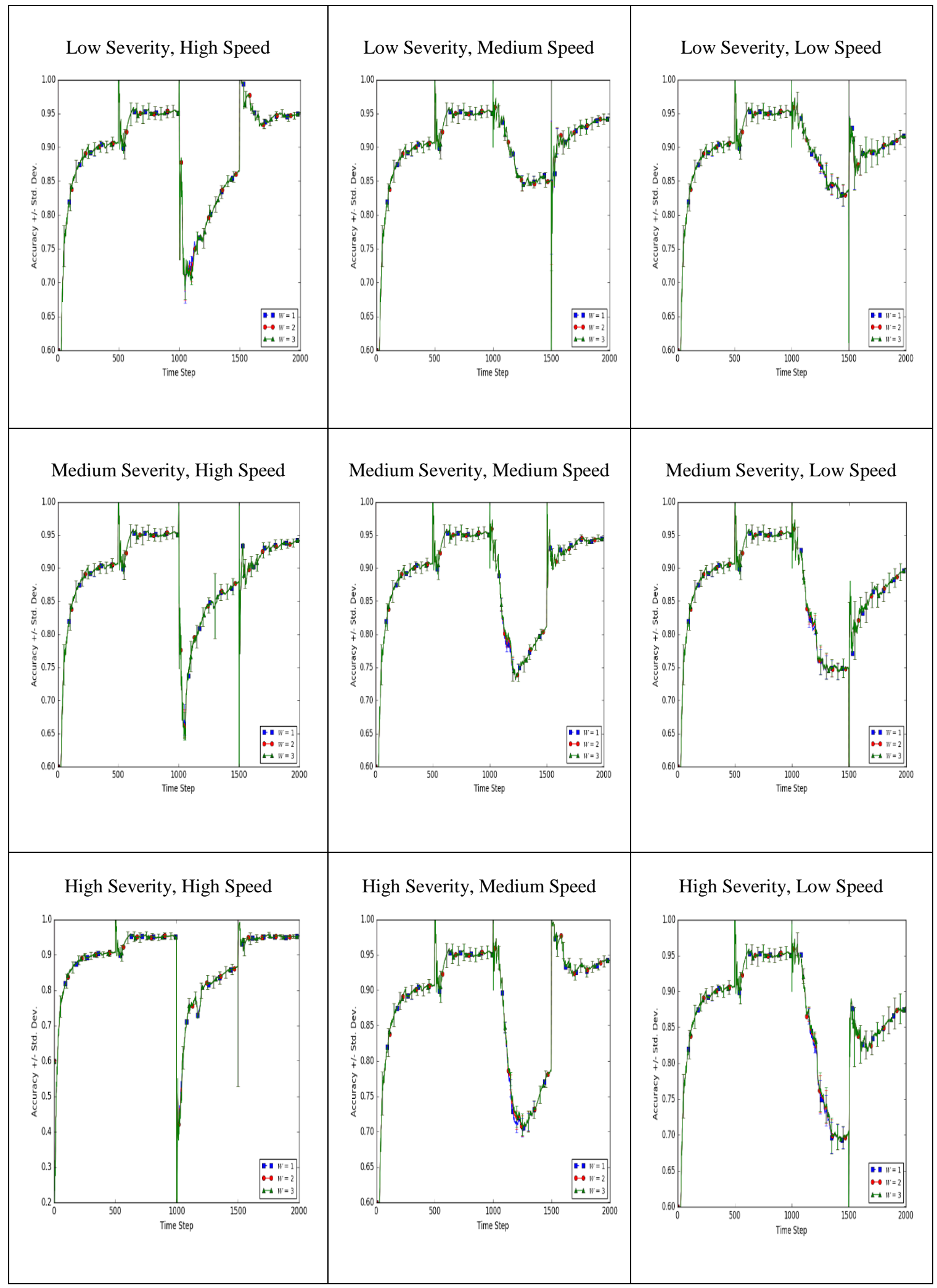




\section{Appendix E - Comparison of Various W Settings using Best MTS - Line}

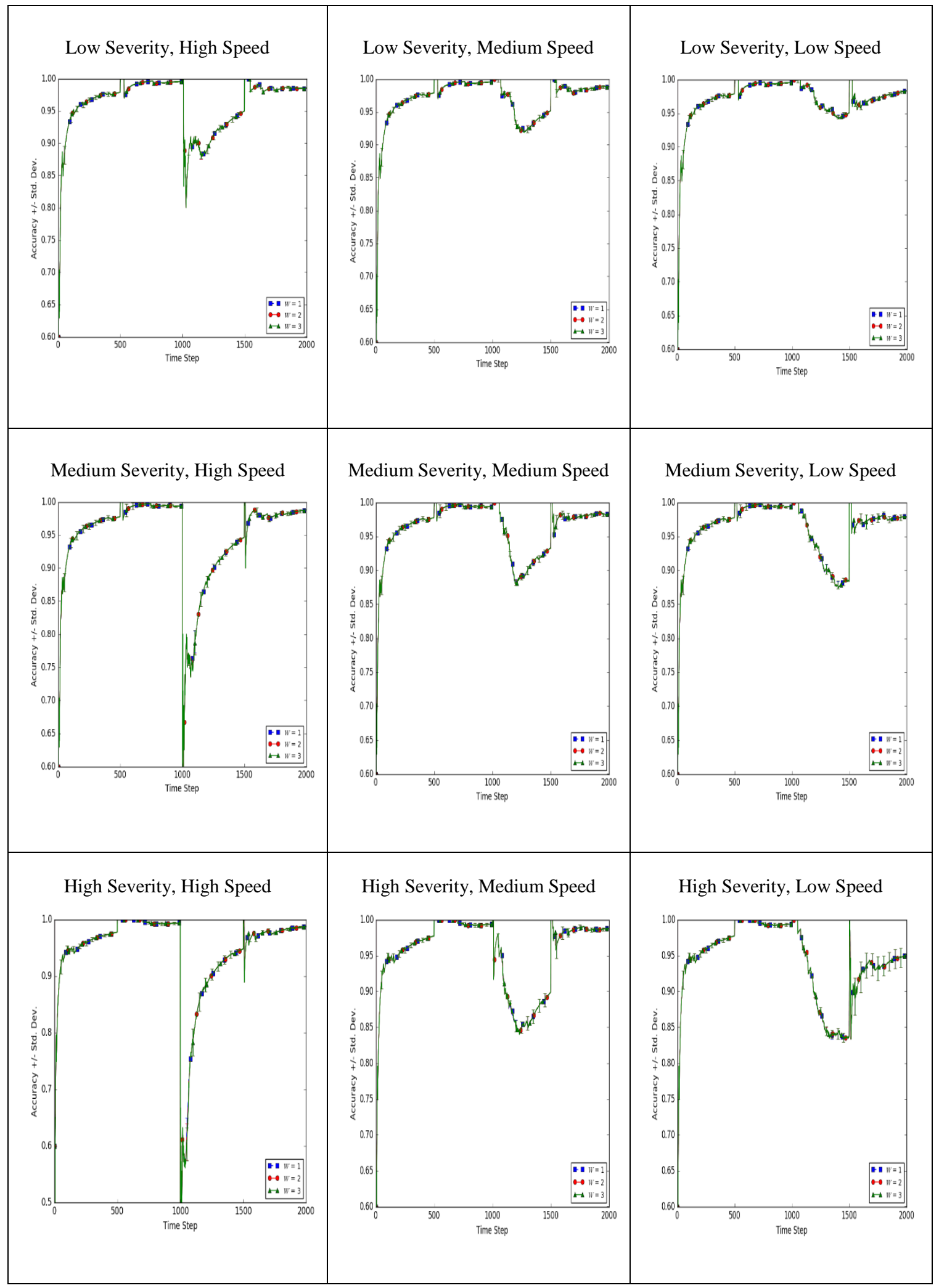


127

Appendix E - Comparison of Various W Settings using Best MTS - Plane

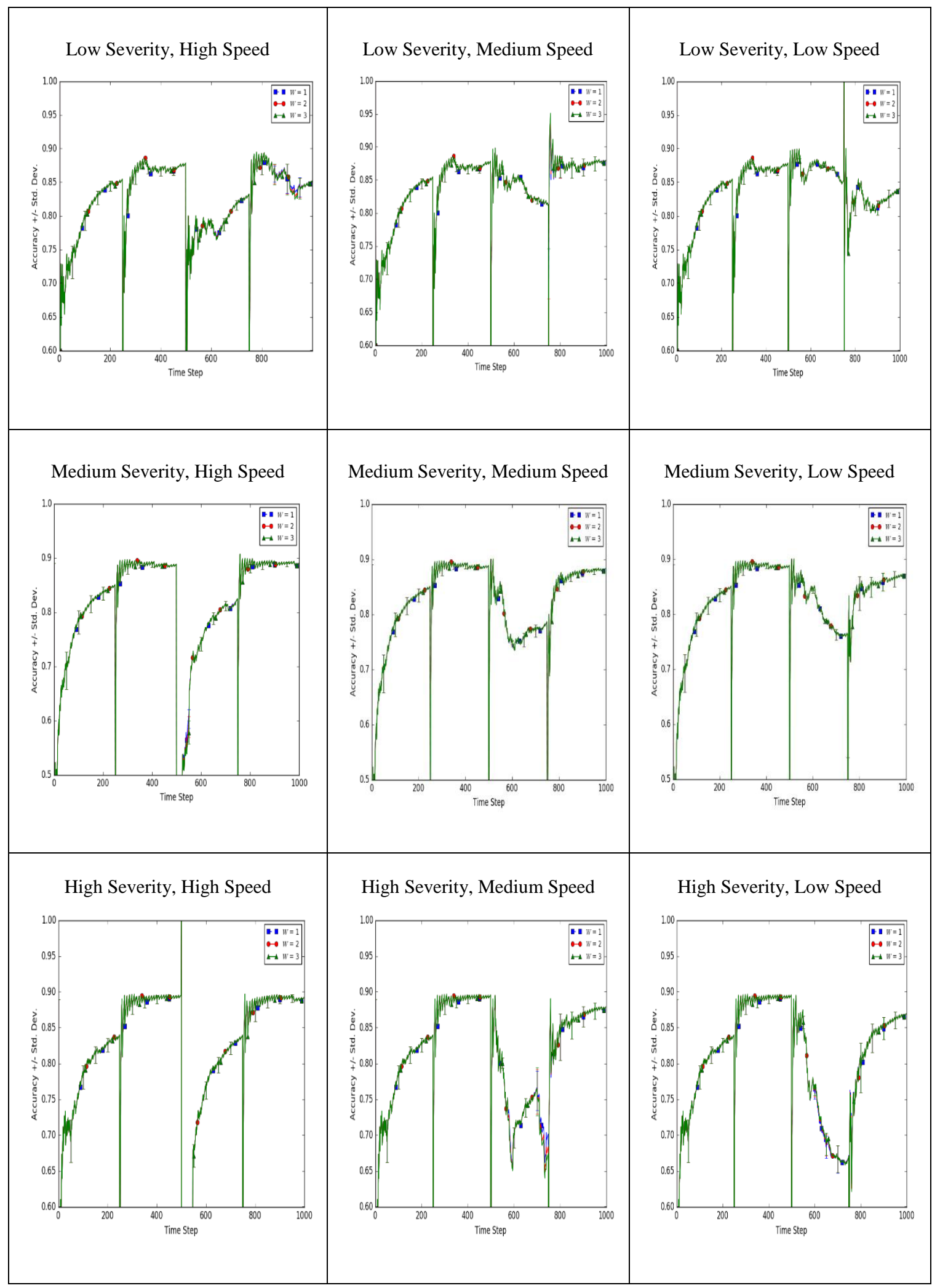




\section{Appendix E - Comparison of Various W Settings using Best MTS - Boolean}

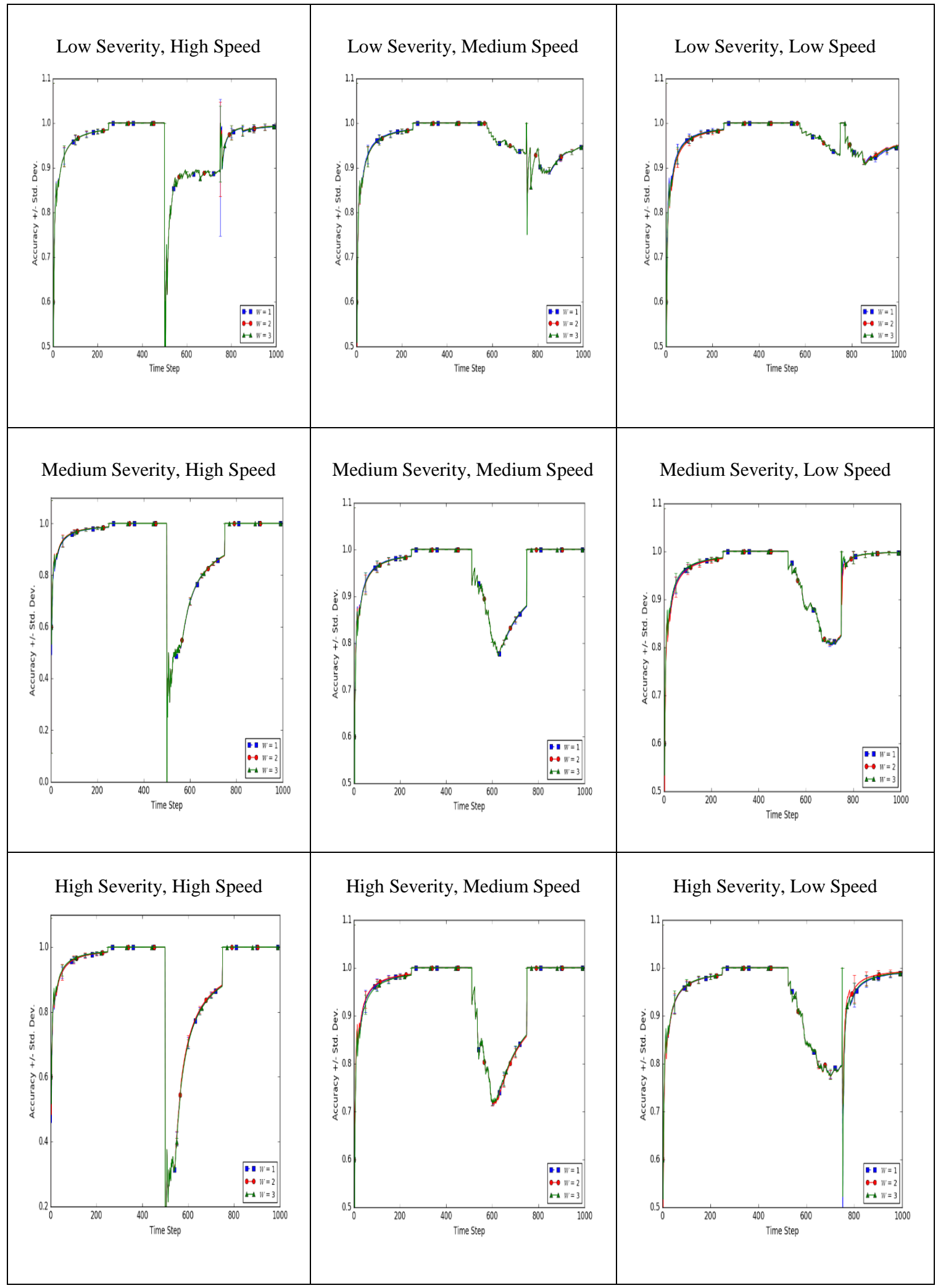


Appendix E - Comparison of Various W Settings using Best MTS - Electricity

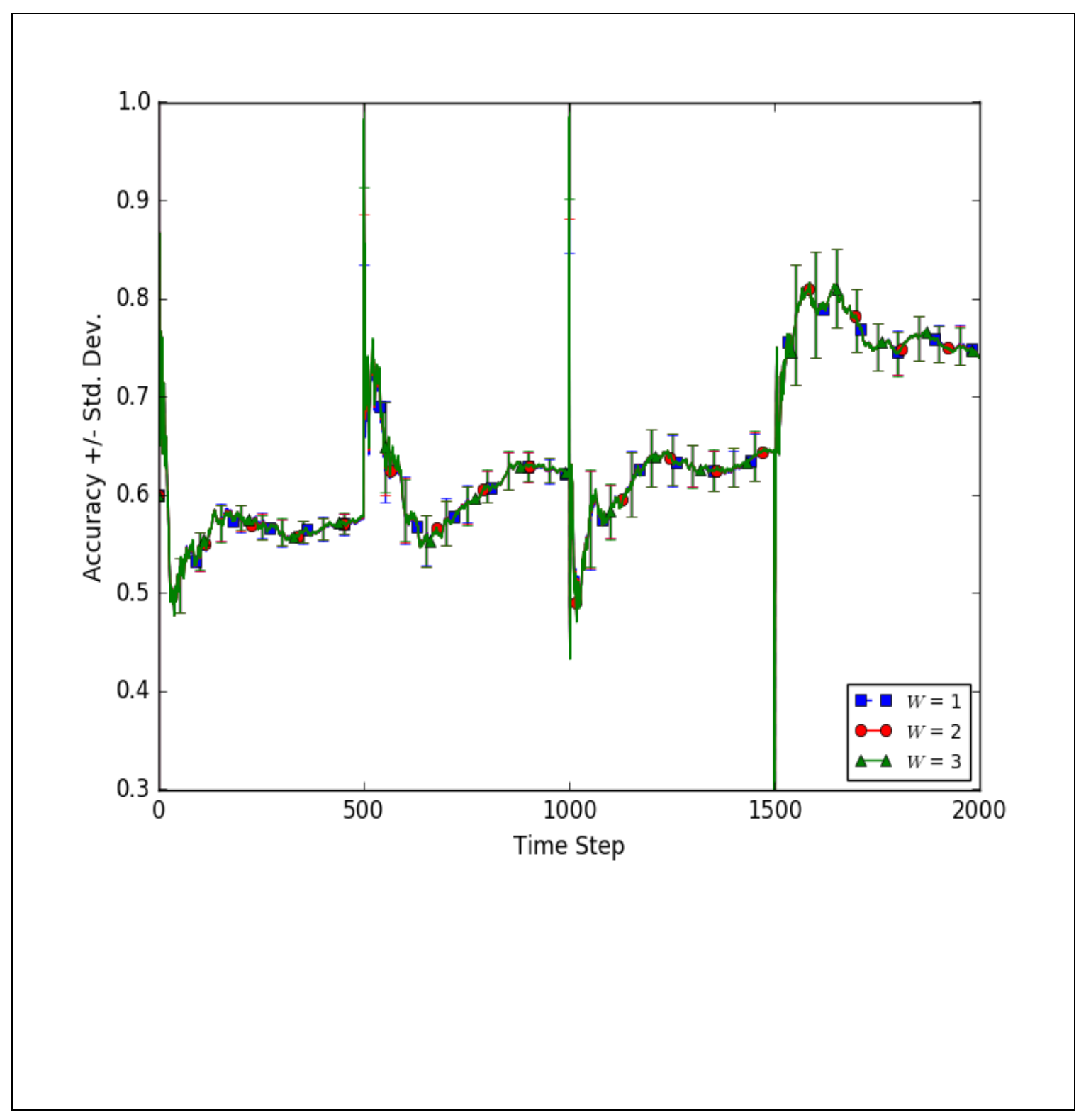


Appendix E - Comparison of Various W Settings using Best MTS Forest CoverType

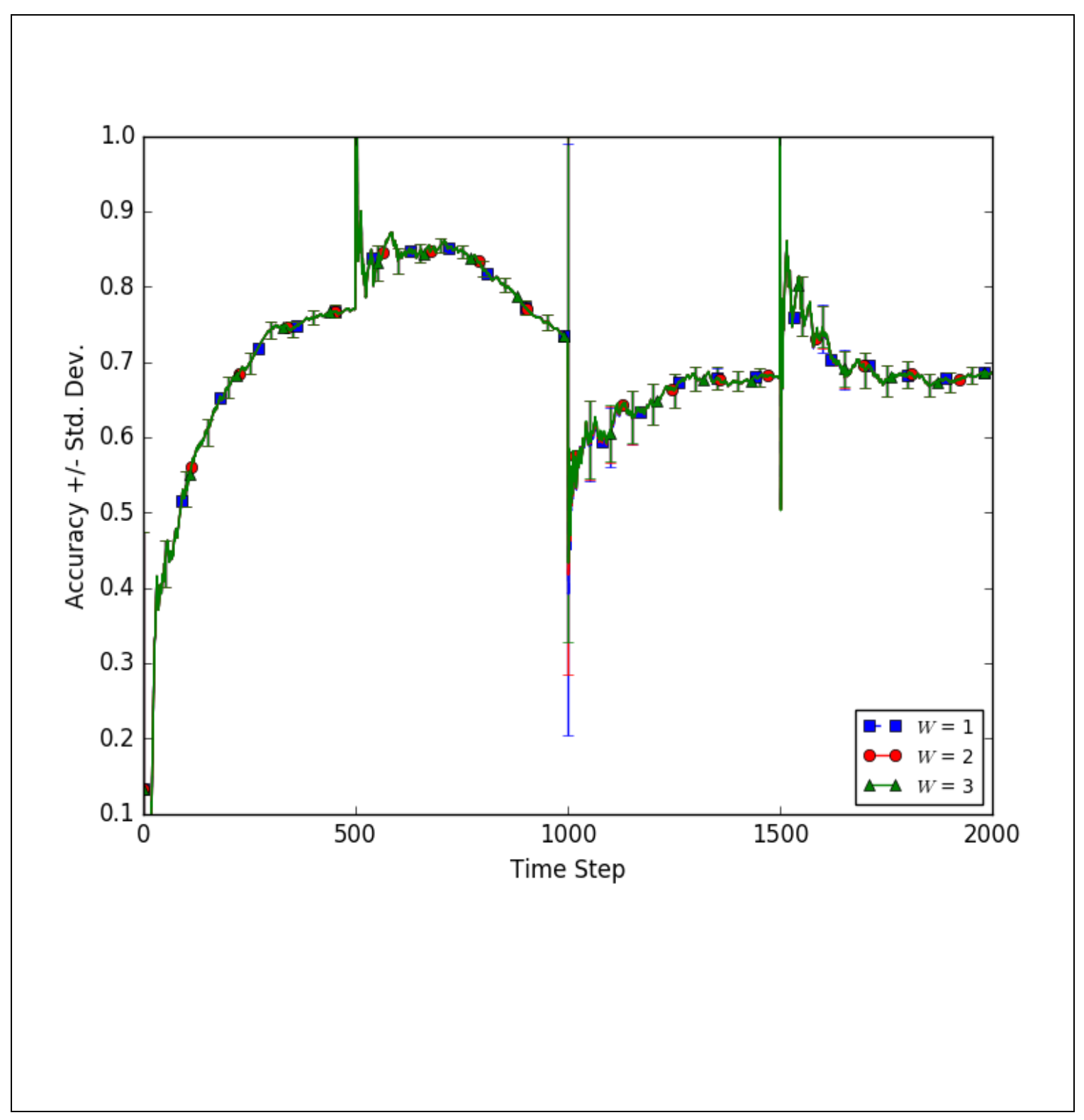


Appendix E - Comparison of Various W Settings using Best MTS - KDD 1999

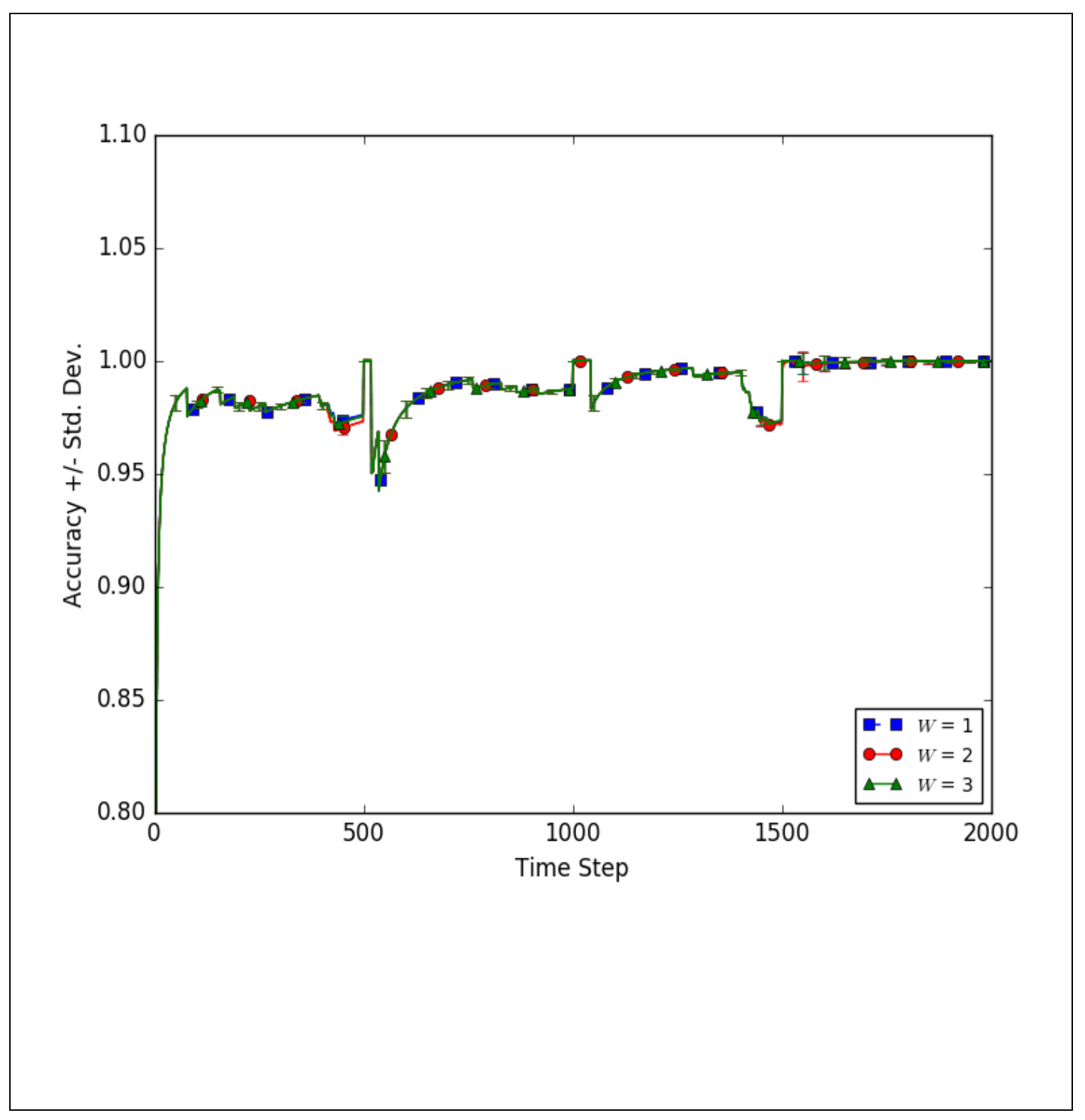




\section{Appendix F - Comparison of Various $W$ Settings using Standard DDD - Circle}

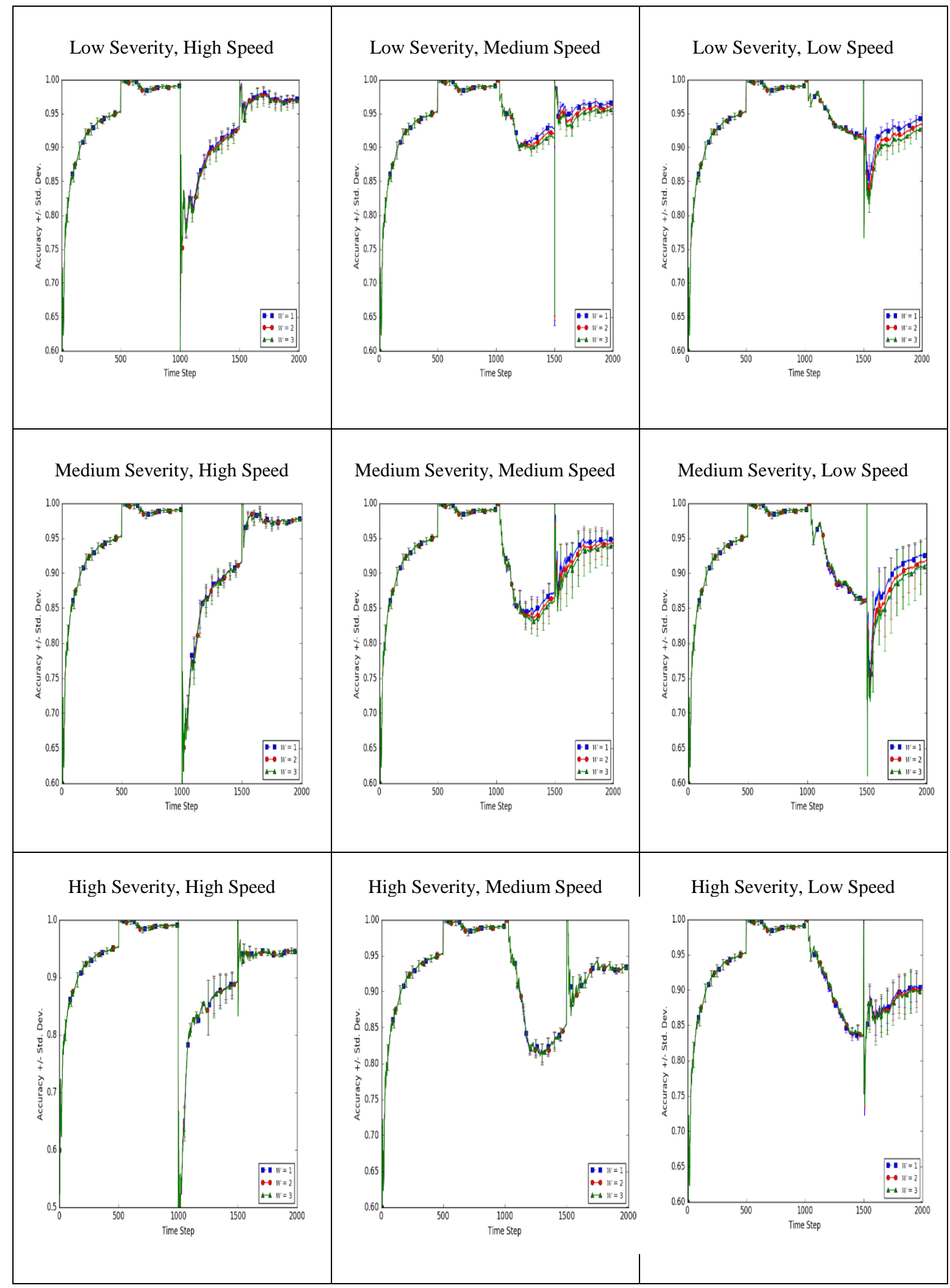




\section{Appendix F - Comparison of Various W Settings using Standard DDD - SineV}

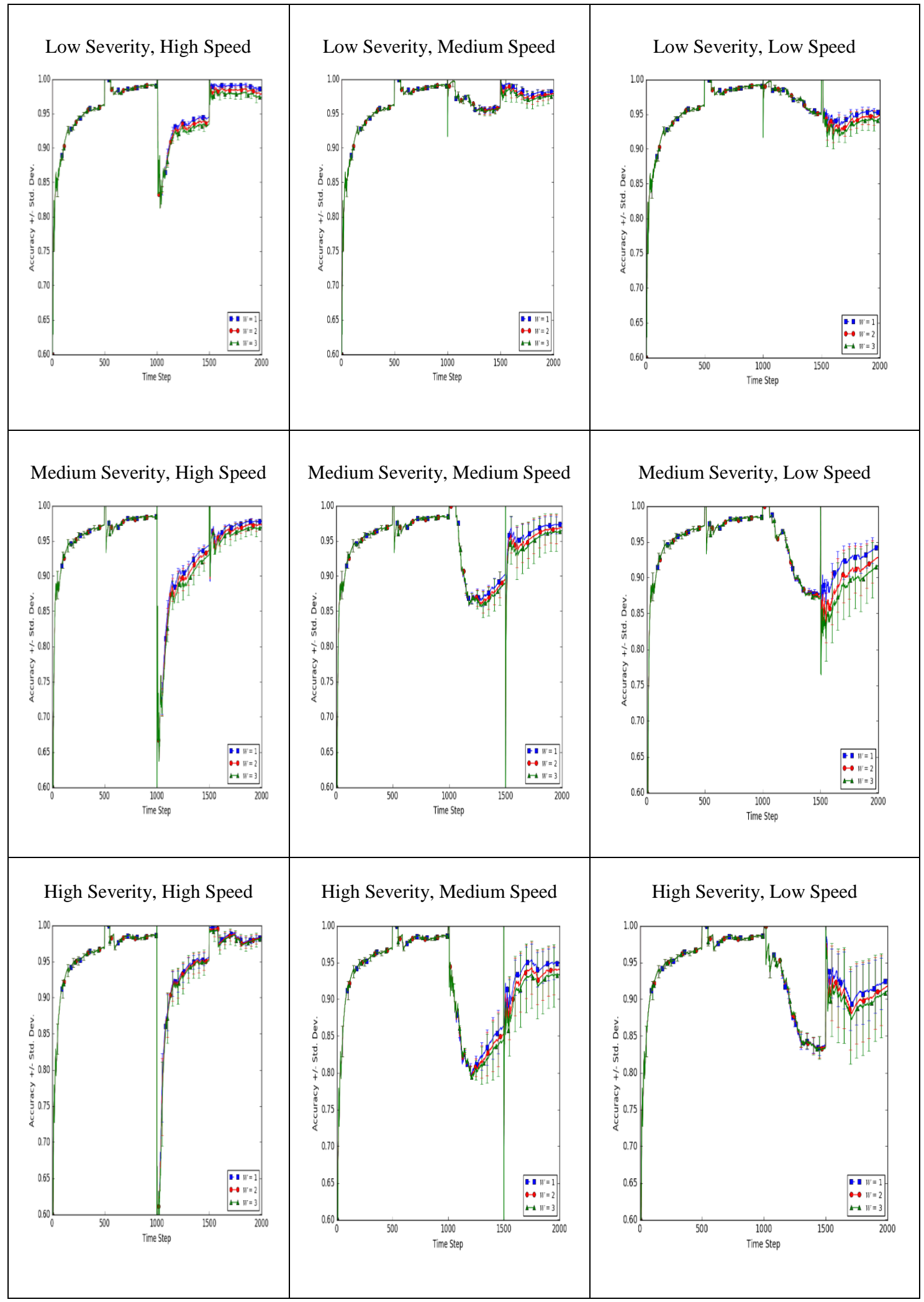


Appendix F - Comparison of Various $W$ Settings using Standard DDD - SineH

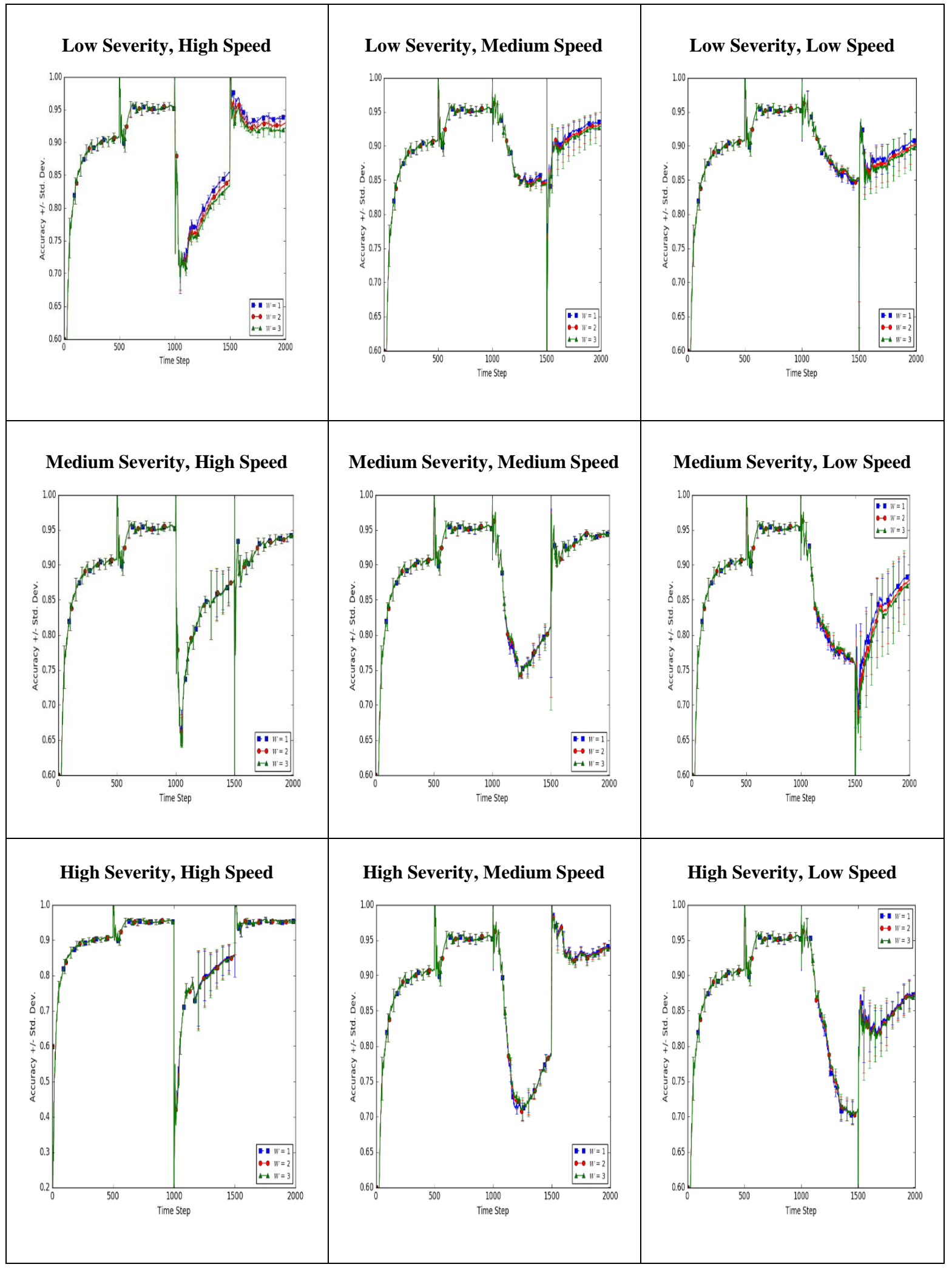




\section{Appendix F - Comparison of Various $W$ Settings using Standard DDD - Line}

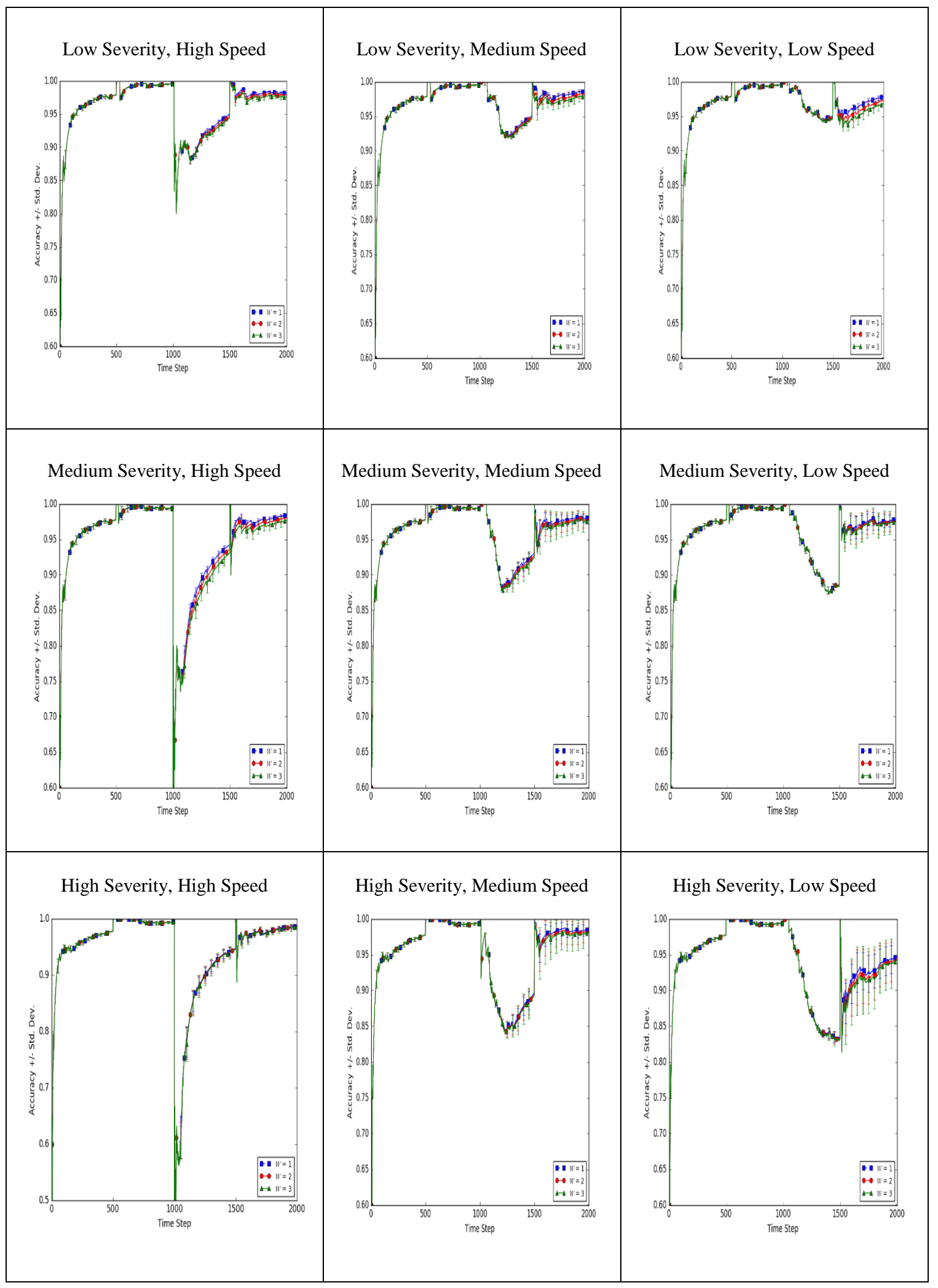


136

Appendix F - Comparison of Various W Settings using Standard DDD - Plane

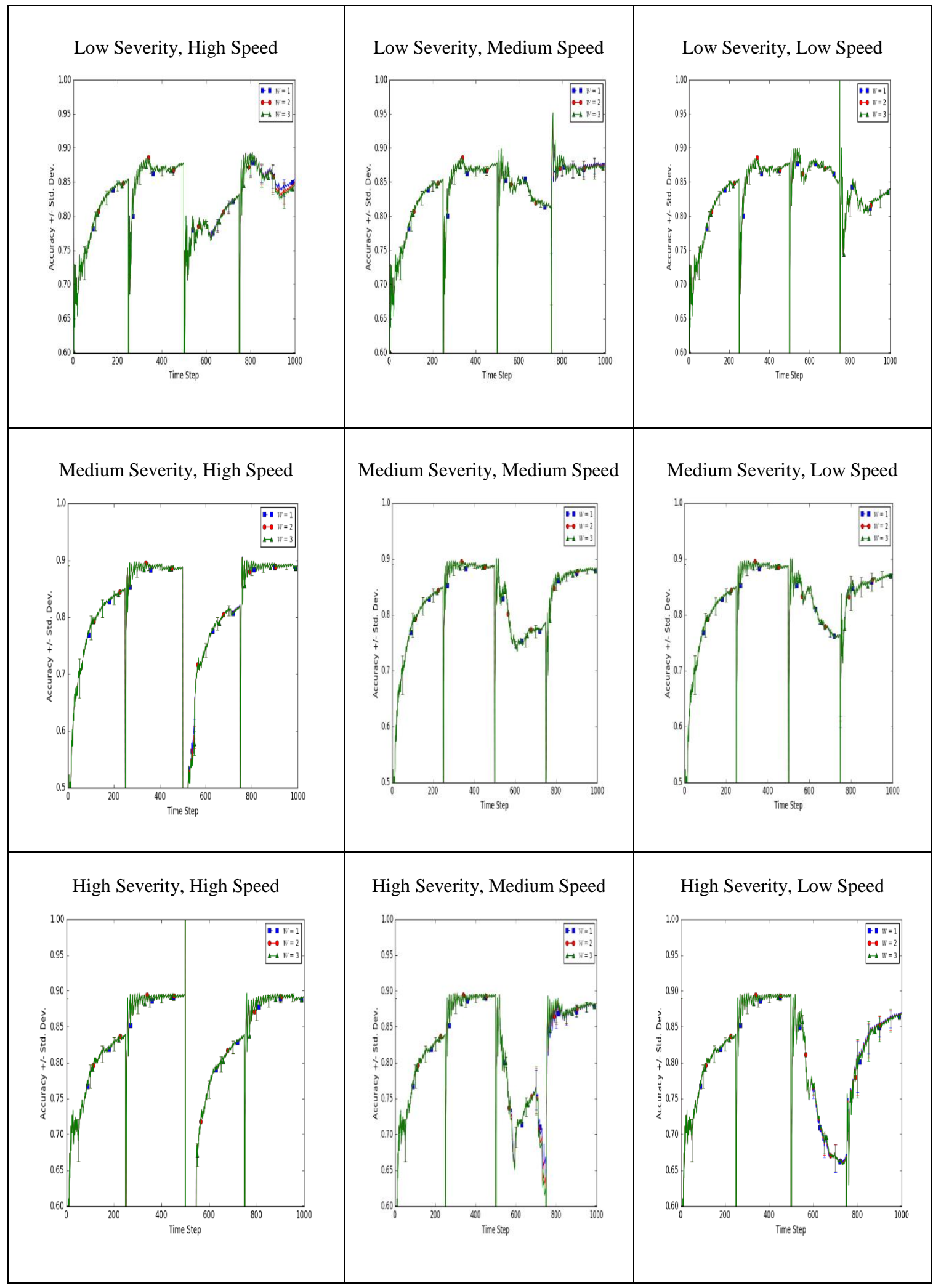




\section{Appendix F - Comparison of Various W Settings using Standard DDD - Boolean}

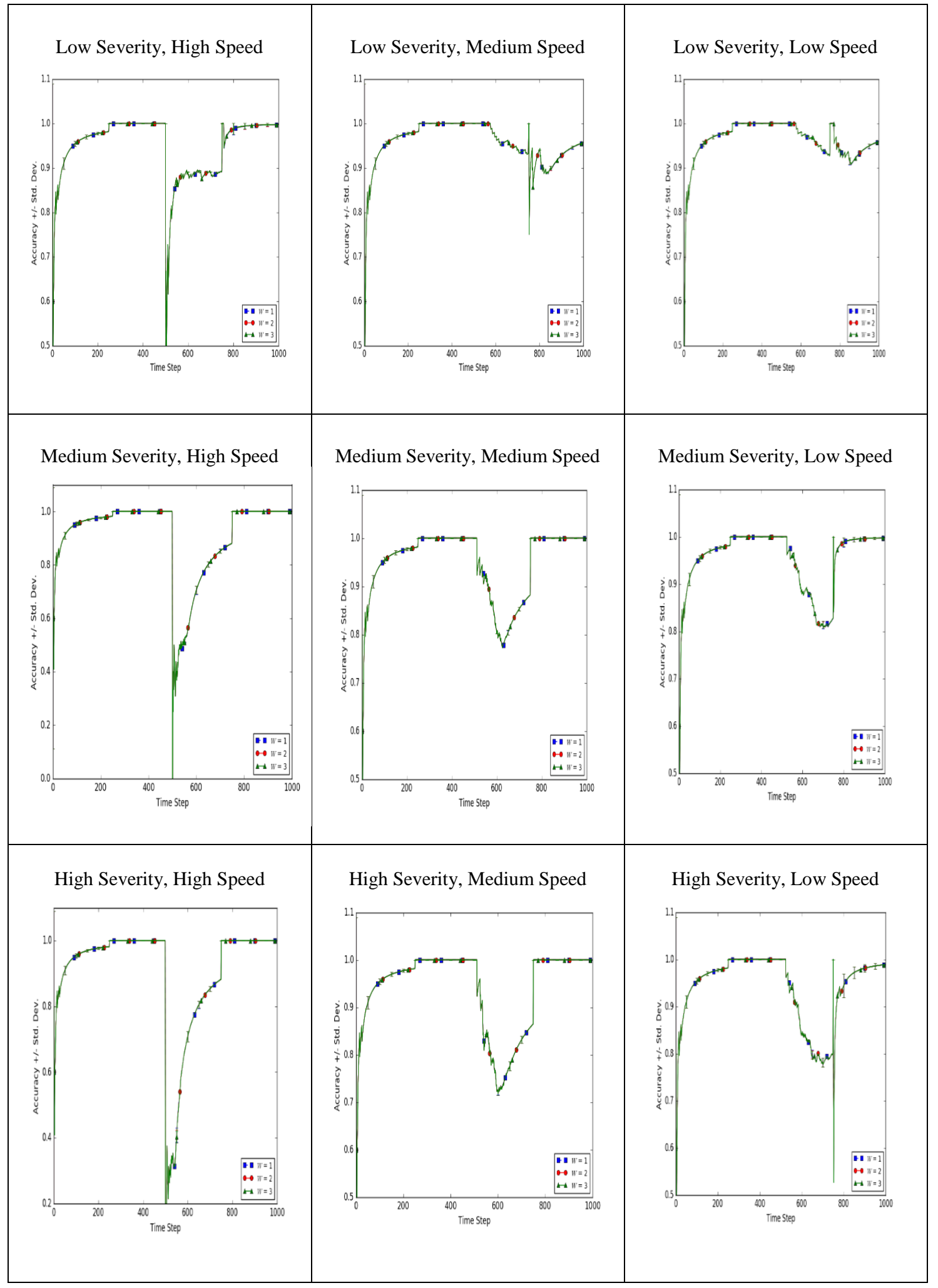


Appendix F - Comparison of Various $W$ Settings using Standard DDD - Electricity

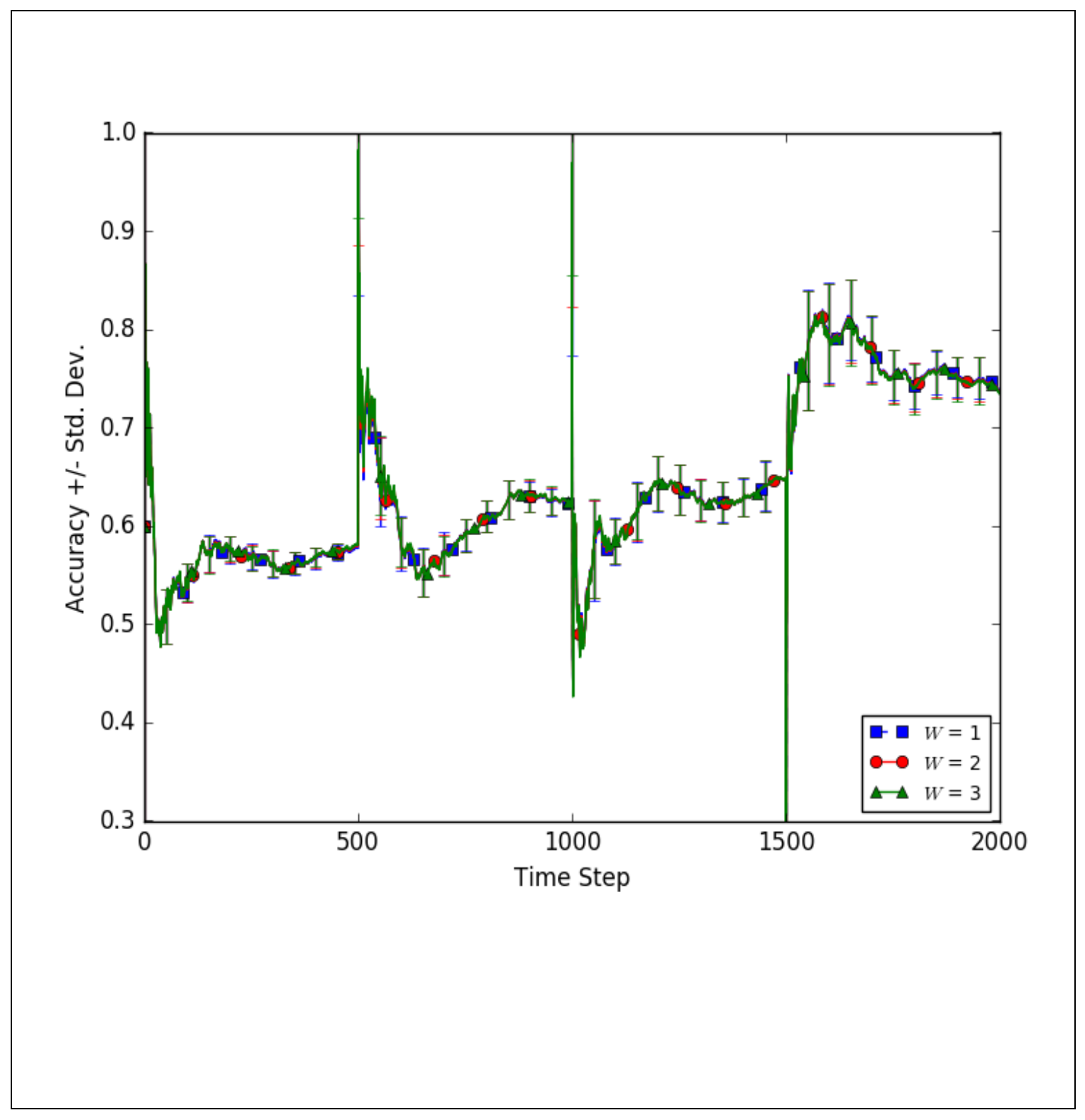


Appendix F - Comparison of Various W Settings using Standard DDD Forest CoverType

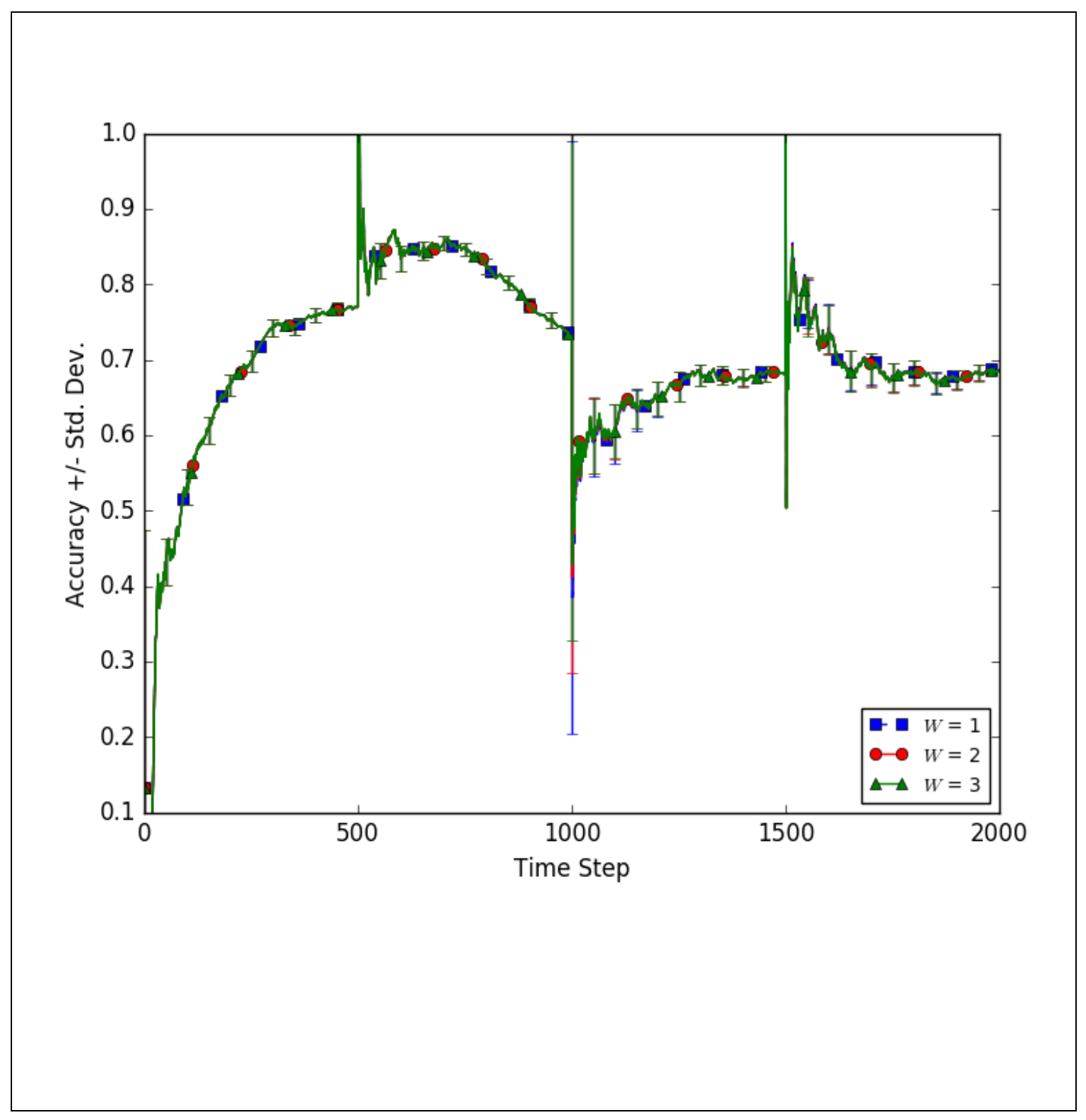


Appendix F - Comparison of Various W Settings using Standard DDD - KDD 1999

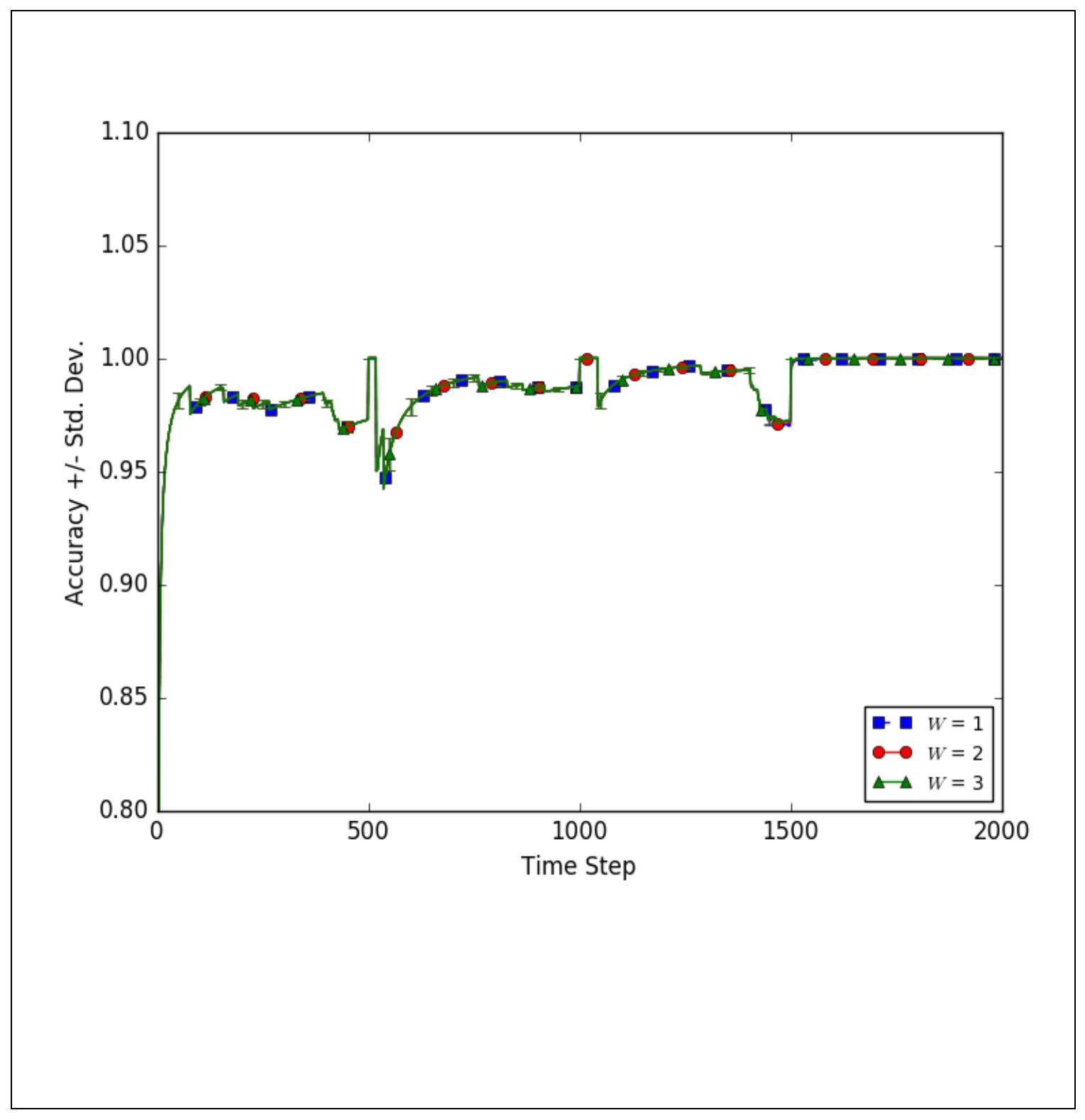




\section{References}

Asuncion, A. \& Newman, D. (2007). UCI machine learning repository.

Baena-García, M., Del Campo-Ávila, J., Fidalgo, R. \& Bifet, A. (2006). Early Drift Detection Method. Proc. Fourth ECML PKDD INt'l Workshop Knowledge Discovery from Data Streams (IWKDDS '06), pp. 77-86.

Bifet, A., Holmes, G., Pfahringer, B. (2010). Leveraging Bagging for Evolving Data Streams. In Proc. of the Eur. Conf. on Mach. Learn. and Knowledge Discovery in Databases (ECMLPKDD). Springer-Verlag, Berlin, 135-150. http://dx.doi.org/10.1007/978-3-642-15880-3_15

Bifet, A., Holmes, G., Pfahringer, B., \& Frank, E. (2010). Fast Perceptron Decision Tree Learning from Evolving Data Streams. In Proc. of the $14^{\text {th }}$ PA Conf. on Knowl. Discov. and Data Mining. Springer-Verlag, Berlin, 135-150. http://dx.doi.org/10.1007/978-3-642-13672-6_30

Blum A. (1997). Empirical Support for Winnow and Weighted-Majority Algorithms: Results on a Calendar Scheduling Domain. Mach. Learn. 26, 1 (1997), 5-23. http://dx.doi.org/10.1023/A:1007335615132

Breiman, L. (2001). Random Forests. Machine Learning. vol. 45, pp.5-32. http://dx.doi.org/10.1023/A:1010933404324

Chandola, V., Banerjee, A. \& Kumar, V. 2009. Anomaly detection: A survey. ACM Comput. Surv. 41, 3, Article 15 July. 58 pages. http://dx.doi.org/10.1145/1541880.1541882

Dawid, A. \& Vovk. V. (1999). Prequential Probability Probability: Principles and Properties. Bernoulli. Vol. 5, no. 1, pp. 125-162. http://dx.doi.org/10.2307/3318616

Dietterich, T. G. (2000). An experimental comparison of three methods for constructing ensembles of decision trees: Bagging, boosting, and randomization. Machine Learning, vol.40, no. 2, pp. 139-157. http://dx.doi.org/10.1023/A:1007607513941

Domingos, P. \& Hulten, G. (2000). Mining high-speed data streams. In Proceedings of the Sixth ACM SIGKDD International Conference on Knowledge Discovery and Data Mining, pages 71-80. ACM Press, New York, NY. http://dx.doi.org/10.1145/347090.347107

Duda, R. O., Hart, P. E., \& Stork, D. G. (2001) Pattern Classification. Wiley. 
Fan, W., Stolfo, S. J., \& Zhang, J. (1999). The application of AdaBoost for distributed, scalable and on-line learning. In Proceedings of the Fifth ACM SIGKDD International Conference on Knowledge Discovery and Data Mining, pages 362366. ACM Press, New York, NY. http://dx.doi.org/10.1145/312129.312283

Fern, A. \& Given, R. (2003). Online Ensemble Learning: An Empirical Study. Machine Learning, vol. 53, pp. 71-109. http://dx.doi.org/10.1023/A:1025619426553

Gao, J., Fan, W., \& Han, J. (2007). On appropriate assumptions to mine data streams: Analysis and practice. In Proc. of IEEE ICDM, Omaha, NE. pp.143-152. http://dx.doi.org/10.1109/icdm.2007.96

Gama, J., Medas, P., Castillo, G., \& Rodrigus, P. (2004). Learning with drift detection. Lecture Notes in Computer Science. 3171. http://dx.doi.org/10.1007/978-3-54028645-5_29

Gama, J., Rocha, R., \& Medas, P. (2003). Accurate decision trees for mining high-speed data stream. In $K D D$ '03, pages 523-528, August. http://dx.doi.org/10.1145/956750.956813

Gama, J., Žliobaitè, I., Bifet, A., Pechenizkiy, M., \& Bouchachia, A. (2014). A survey on concept drift adaptation. ACM Comput. Surv. 46, 4, Article 44, (March), 37 pages. http://dx.doi.org/10.1145/2523813

Han, J., Kamber, M., \& Pei, J. (2012). In Data Mining: concepts and techniques. Morgan Kaufmann. http://dx.doi.org/10.1007/978-1-4419-1428-6_3752

Harries, M. (1999). Splice-2 Comparative Evaluation: Electricity Pricing. Technical Report UNSW-CSE-TR-9905, Artificial Intelligence Group, School of Computer and Eng., The Univ. of New South Wales, Sydney.

Hulten, G., Spencer, L. \& Domingos, P. (2001). Mining time-changing data streams. In Proceedings of the Seventh ACM SIGKDD International Conference on Knowledge Discovery and Data Mining, pages 97-106. ACM Press, New York, NY. http://dx.doi.org/10.1145/502512.502529

Kelly, M. G., Hand, D. J., \& Adams, N. M. (1999). The Impact of Changing Populations on Classifier Performance. In Proc. of the $5^{\text {th }}$ ACM SIGKDD Int. Conf. on Knowl. Disc. and Dat. Mining (KDD). ACM, 367-371. http://dx.doi.org/10.1145/312129.312285

Klenner, M. \& Hahn. U. (1994). Concept versioning: A methodology for tracking evolutionary concept drift in dynamic concept systems. In Proceedings of the Eleventh European Conference on Knowledge Discovery and Data Mining. Pages 473-477. John Wiley \& Sons, London. 
Klinkenberg, R. \& Joachims, T. (2000). Detecting concept drift with support vector machines. In Proceedings of the Seventeenth International Conference on Machine Learning, pages 487-494. Morgan Kaufmann, San Francisco, CA.

Kolter, J. \& Maloof, M. (2007). Dynamic Weighted Majority: An Ensemble Method for Drifting Concepts. Journal of Machine Learning Research 8, 2755-2790.

Kuncheva, L.I. \& Whitaker, C.J. (2003). Measures of Diversity in Classifier Ensembles and Their Relationship with the Ensemble Accuracy. Machine Learning, vol. 51, pp. 181-207. http://dx.doi.org/10.1023/A:1022859003006

Littlestone, N. \& Warmuth, M. K. (1994). The Weighted Majority Algorithm. Information and Computation. 108, (2):212-261. http://dx.doi.org/10.1006/inco.1994.1009

Minku, L., White, A., \& Yao, X. (2010). The Impact of Diversity on Online Ensemble Learning in the Presence of Concept Drift. IEEE Transactions on Knowledge and Data Engineering 22, 730-742. Issue 5. http://dx.doi.org/10.1109/TKDE.2009.156

Minku, L. \& Yao, X. (2008). On-line Bagging Negative Correlation Learning. Proc. Int'l Joint Conf. Neural Networks (IJCNN'08), pp 1375-1382. http://dx.doi.org/10.1109/ijenn.2008.4633977

Minku, L. \& Yao, X. (2012). DDD: A new Ensemble Approach for Dealing with Concept Drift. IEEE Transactions on Knowledge and Data Engineering 24,4, 619-633. http://dx.doi.org/10.1109/TKDE.2011.58

Narasimhamurthy, A. \& Kuncheva, L.I. (2007). A Framework for Generating Data to Simulate Changing Environments, Proc. $25^{\text {th }}$ IASTED Int'l Multi-Conf.: Artificial Intelligence and Applications, pp. 384-389.

Oza, N.C. \& Russell S. (2001). Experimental Comparisons of Online and Batch Version of Bagging and Boosting. Proc. ACM SIGKDD Int'l Conf. Knowledge Discovery and Data Mining, pp. 359-364. http://dx.doi.org/10.1145/502512.502565

Schlimmer, J.C. \& Granger, R. H. (1986). Beyond incremental processing: Tracking concept drift. In Proceeding of the Fifth National Conference on Artificial Intelligence, pages 502-507. AAAI Press, Menlo Park, CA.

Scholz, M. \& Klinkenberg, R. (2006). Boosting Classifiers for Drifting Concepts. Technical report, Collaborative Research Center on the Reduction of Complexity for Multivariate Data Structures (SFB 475), University of Dortmund, Germany, January. 
Street, W.N. \& Kim, Y. (2001). A streaming ensemble algorithm (SEA) for large-scale classification. In Proceedings of the Seventh ACM SIGKDD International Conference on Knowledge Discovery and Data Mining, pages 377-382. ACM Press, New York, NY. http://dx.doi.org/10.1145/502512.502568

The UCI KDD Archieve. (1999). http://mlr.cs.umass.edu/ml/databases/kddcup99/kddcup99.html.

Tsymbal, A. (2004). The Problem of Concept Drift: Definitions and Related Work. Tech. rep. Department of Computer Science, Trinity College, Dublin.

Utgoff, P., Berkman, N. \& Clouse, J. (1997). Decision Tree Induction Based on Efficient Tree Restructuring. Machine Learning, vol. 29, no. 1, pp. 5-44. http://dx.doi.org/10.1023/A:1007413323501

Wang, H., Fan, W., Yu, P.S., \& Han, J. (2003). Mining concept-drifting data streams using ensemble classifiers. In Proceedings of the Ninth ACM SIGKDD International Conference on Knowledge Discovery and Data Mining, pages 226235. ACM Press, New York, NY. http://dx.doi.org/10.1145/956750.956778

Widmer, G. (1997). Tracking context changes through meta-learning. Machine Learning, 27:259-286. http://dx.doi.org/10.1023/A:1007365809034

Widmer, G. \& Kubat, M. (1993). Effective Learning in Dynamic Environments by Explicit Context Tracking. In Proc. of the Eur. Conf. on Mach. Learn. (ECML). Springer, Berlin, 227-243. http://dx.doi.org/10.1007/3-540-56602-3_139

Widmer, G. \& Kubat, M. (1996). Learning in the presence of concept drift and hidden contexts. Machine Learning, 23:69-101. http://dx.doi.org/10.1007/BF00116900

Yule, G. (1900). On the Association of Attributes in Statistics. Philosophical Trans. Royal Soc. of London, Series A, vol. 194, pp. 257-319. http://dx.doi.org/10.1098/rsta.1900.0019

Žliobaitè, I. (2011). Controlled Permutations for Testing Adaptive Classifiers. In Proc. of the $14^{\text {th }}$ Int. Conf. on Discovery Science (DS). Springer, Berlin, 365-379. http://dx.doi.org/10.1007/978-3-642-24477-3_29 